\title{
Guangtian Zhu
}

B.S. Physics, Tsinghua University, 2007

\author{
Submitted to the Graduate Faculty of \\ The Department of Physics and Astronomy in partial fulfillment \\ of the requirements for the degree of \\ Doctor of Philosophy
}

University of Pittsburgh 


\author{
UNIVERSITY OF PITTSBURGH \\ SCHOOL OF ARTS AND SCIENCES \\ DEPARTMENT OF PHYSICS AND ASTRONOMY
}

This dissertation was presented

by

Guangtian Zhu

It was defended on

March 2, 2011

and approved by

Dr. Rainer Johnsen, Professor, Department of Physics and Astronomy

Dr. Robert Devaty, Associate Professor, Department of Physics and Astronomy

Dr. Arthur Kosowsky, Associate Professor, Department of Physics and Astronomy

Dr. Larry Shuman, Professor, Department of Industrial Engineering

Dissertation Advisor: Dr. Chandralekha Singh, Associate Professor, Department of Physics and Astronomy 
Copyright (c) by Guangtian Zhu 2011 


\title{
IMPROVING STUDENTS’ UNDERSTANDING OF QUANTUM MECHANICS
}

\author{
Guangtian Zhu, $\mathrm{PhD}$ \\ University of Pittsburgh, 2011
}

Learning physics is challenging at all levels. Students' difficulties in the introductory level physics courses have been widely studied and many instructional strategies have been developed to help students learn introductory physics. However, research shows that there is a large diversity in students' preparation and skills in the upper-level physics courses and it is necessary to provide scaffolding support to help students learn advanced physics. This thesis explores issues related to students' common difficulties in learning upper-level undergraduate quantum mechanics and how these difficulties can be reduced by research-based learning tutorials and peer instruction tools. We investigated students' difficulties in learning quantum mechanics by administering written tests and surveys to many classes and conducting individual interviews with a subset of students. Based on these investigations, we developed Quantum Interactive Learning Tutorials (QuILTs) and peer instruction tools to help students build a hierarchical knowledge structure of quantum mechanics through a guided approach. Preliminary assessments indicate that students' understanding of quantum mechanics is improved after using the researchbased learning tools in the junior-senior level quantum mechanics courses. We also designed a standardized conceptual survey that can help instructors better probe students' understanding of quantum mechanics concepts in one spatial dimension. The validity and reliability of this quantum mechanics survey is discussed. 
TABLE OF CONTENTS

PREFACE - ACKNOWLEDGMENT XIII

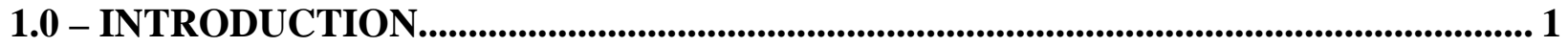

1.1 QUANTUM MECHANICS VS. CLASSICAL MECHANICS............................ 4

1.2 A SHORT REVIEW OF RELEVANT PHYSICS EDUCATION RESEARCH

(PER)

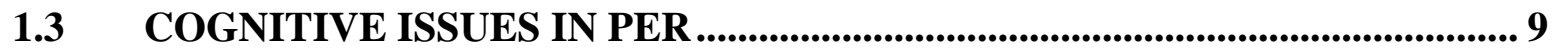

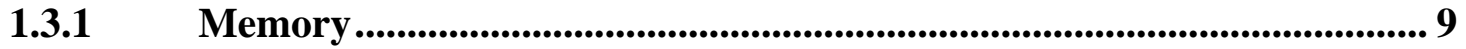

1.3.2 Metacognition \& Epistemology .................................................. 12

1.3.3 Optimal Mismatch. ..................................................................... 14

Zone of Proximal Development (ZPD)............................................. 15

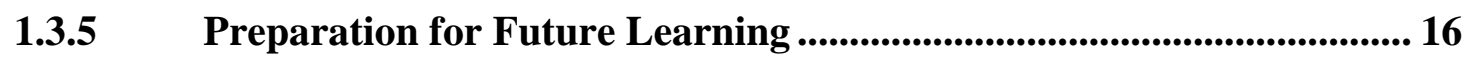

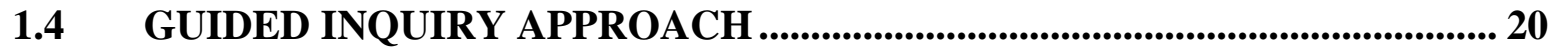

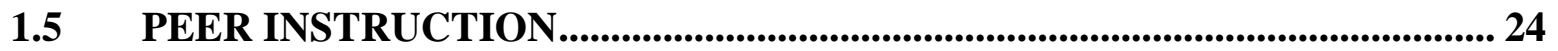

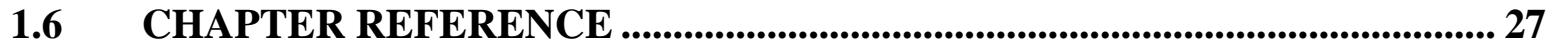

$2.0 \quad-\quad$ IMPROVING STUDENTS' UNDERSTANDING OF POSSIBLE

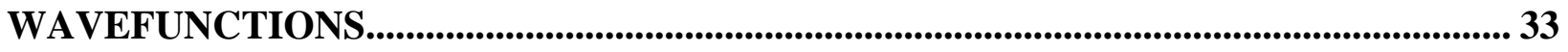

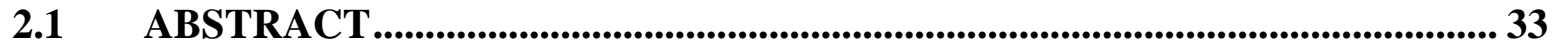

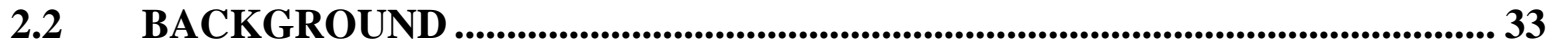


$2.3 \quad$ INVESTIGATION OF STUDENTS’ DIFFICULTIES 36

2.3.1 Difficulties related to the normalization of possible wavefunctions.... 36

2.3.2 Difficulties related to the boundary conditions in different potential energy wells. 38

2.3.3 Difficulties related to the continuity of possible wavefunction .............40

2.3.4 Difficulties related to the continuity of the first derivative of a possible wavefunction. 41

2.3.5 Difficulties with qualitative sketch of the possible wavefunction without using quantitative solutions.............................................................................. 42

$2.4 \quad$ RESEARCH BASED LEARNING TOOLS............................................................. 43

2.4.1 Possible wavefunction for a 1D infinite square well ................................. 44

2.4.2 Possible wavefunction for a 1D finite square well..................................... 48

2.4.3 Possible wavefunction for a free particle .................................................... 51

2.4.4 QuILT Homework for Possible Wavefunctions......................................... 52

$2.5 \quad$ PRE-TEST AND POST-TEST DATA ................................................................. 53

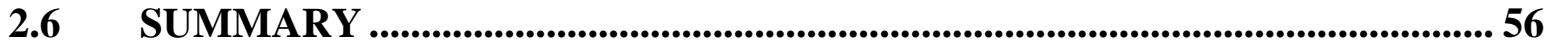

2.7 CHAPTER REFERENCE ...........................................................................56

3.0 - IMPROVING STUDENTS' UNDERSTANDING OF BOUND AND SCATTERING

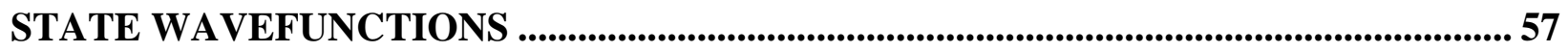

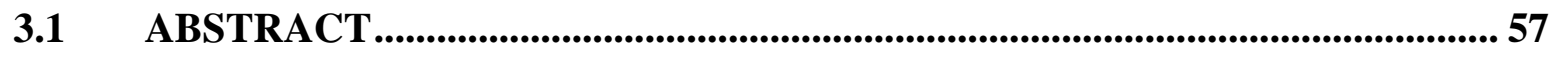

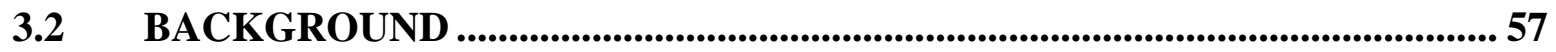

INVESTIGATION OF STUDENT'S DIFFICULTIES ........................................ 58 
3.3.1 Difficulties related to the classical bound state and the quantum bound and scattering states. 59

3.3.2 Difficulties related to the bound and scattering states being part of the same wavefunction 60

3.3.3 Difficulties related to the quantum tunneling effect ............................. 62

3.3.4 Difficulties in determining bound states related to the maximum value of potential energy vs. the energy of the particle

3.3.5 Difficulties related to the directional preference of the scattering state wavefunctions for a symmetric potential energy 64

3.3.6 Difficulties related to the transmission and reflection of a quantum mechanical particle in the scattering state. 65

$\begin{array}{llllll}3.4 & \text { RESEARCH-BASED } & \text { LEARNING TUTORIAL } & \text { FOR } & \text { BOUND }\end{array}$ SCATTERING STATES WAVEFUNCTION.................................................................66

3.4.1 Warm-up tutorial for the bound \& scattering state wavefunction ..... 67

3.4.2 QuILT of the bound \& scattering state wavefunction............................. 68

P.5 PRELIMINARY EVALUATION ................................................................... 71

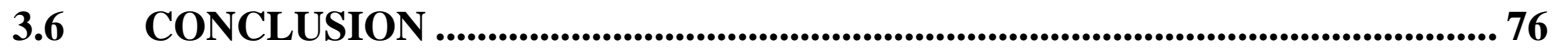

3.7 CHAPTER REFERENCE ................................................................................... 76

4.0 - IMPROVING STUDENTS' UNDERSTANDING OF DRAWING ENERGY EIGENFUNCTIONS ......................................................................................................................... 77

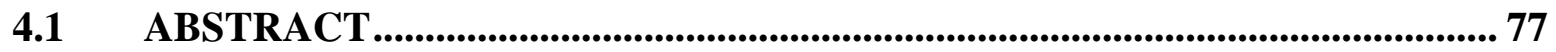

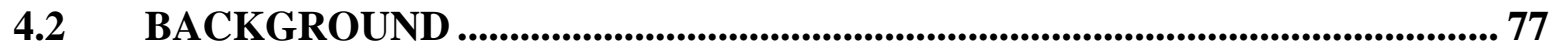

$4.3 \quad$ INVESTIGATION OF STUDENT'S DIFFICULTIES ......................................... 79 
4.3.1 Difficulties related to the position of the $\psi(x)=0$ axis . 79

4.3.2 Difficulties related to decaying and oscillatory wavefunctions............ 80

4.3.3 Difficulties related to the wavelength of oscillatory wavefunctions .... 82 4.3.4 Difficulties related to the absolute value of the amplitude of wavefunctions ............................................................................................................ 83

4.4 RESEARCH-BASED LEARNING TUTORIAL FOR DRAWING ENERGY EIGENFUNCTIONS............................................................................................... 85

4.5 PRELIMINARY EVALUATION ................................................................. 88

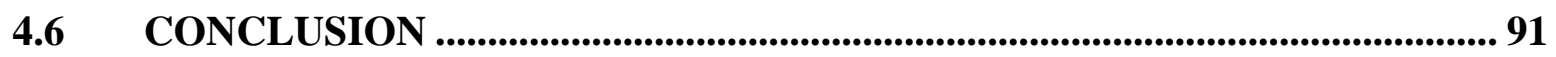

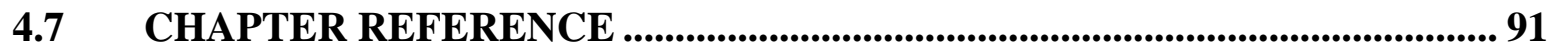

5.0 - IMPROVING STUDENTS' UNDERSTANDING OF QUANTUM MEASUREMENT 92

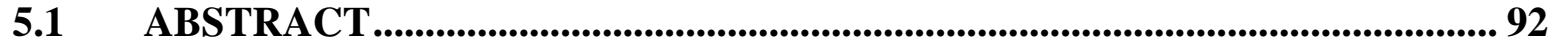

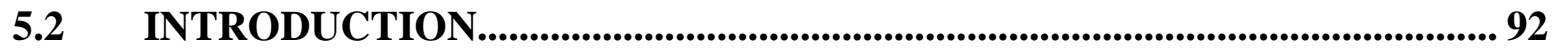

$5.3 \quad$ BACKGROUND ..................................................................................................... 93

INVESTIGATION OF STUDENTS’ DIFFICULTIES ................................. 95

5.4.1 Difficulty in Distinguishing between Eigenstates of Operators corresponding to Different Observables ............................................................... 95

D.4.2 Difficulty with possible outcomes of a measurement........................... 97

5.4.3 Difficulty with the probability of measuring energy .......................... 99

5.4.4 Measurement and future time evolution of the wavefunction ........... 102

5.4.5 An operator acting on a state corresponds to a measurement of the

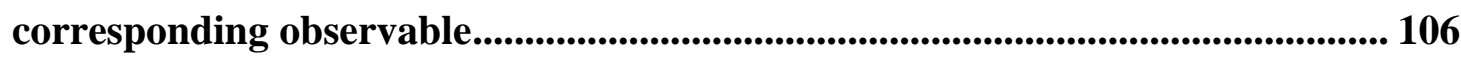


5.5 WARM-UP FOR THE QUILT ON QUANTUM MEASUREMENT . 109

5.6 QUILT FOR QUANTUM MEASURMENT PART I ..................................... 111

5.6.1 Outcome of Quantum Measurement........................................................... 113

5.6.2 Calculating the Probability of Measuring Different Values ............... 117

5.7 QUILT FOR QUANTUM MEASURMENT PART II.................................... 120

5.7.1 Energy Measurement..................................................................................... 121

5.7.2 Position Measurement ........................................................................... 123

5.7.3 Time Dependence of the Measurement Probability ............................... 125

5.7.4 Consecutive Measurements.................................................................... 127

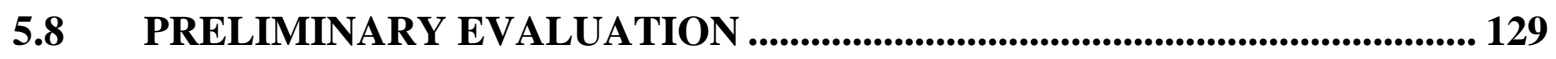

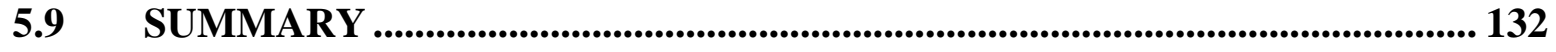

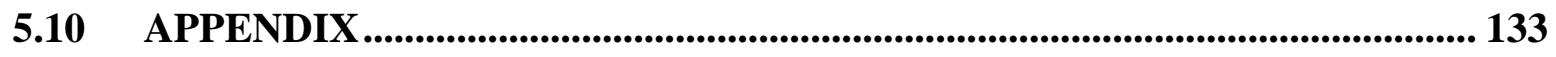

5.11 CHAPTER REFERENCES ............................................................................... 134

6.0 - IMPROVING STUDENS' UNDERSTANDING OF QUANTUM MECHANICS VIA

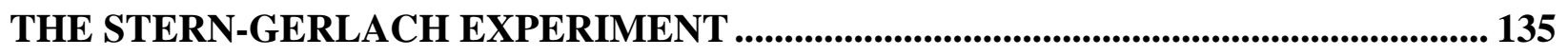

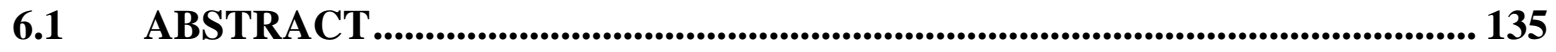

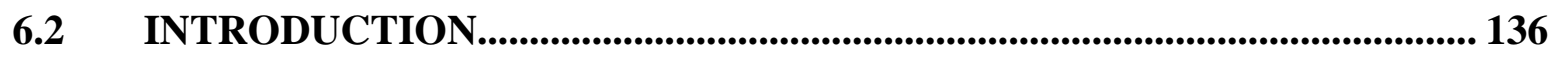

6.3 INVESTIGATION OF STUDENTS' DIFFICULTIES WITH STERN-

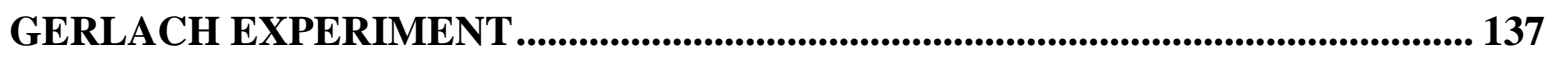

6.3.1 Difficulty in Distinguishing between the Physical Space and Hilbert

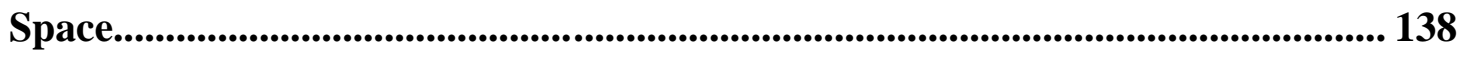

6.3.2 Difficulty in Determining the Pattern on the Screen with Particles in Different Spin States Passing through a SGA ............................................................. 139 
6.3.3 Larmor Precession of Spin involves Precession in Physical Space.... 143

6.3.4 Difficulty with State Preparation ............................................................. 144

6.3.5 Difficulty in Differentiating between a Superposition and a

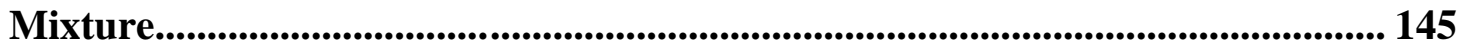

6.4 SGE QUILT: WARM-UP AND HOMEWORK.............................................. 146

6.5 SGE QUILT ............................................................................................................... 150

6.6 PRELIMINARY EVALUATION ................................................................ 155

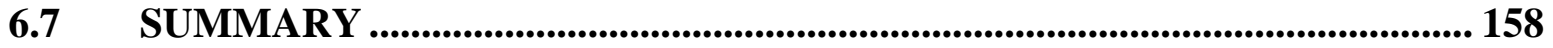

6.8 APPENDIX: THE PRE-/POST-TEST QUESTIONS ....................................... 159

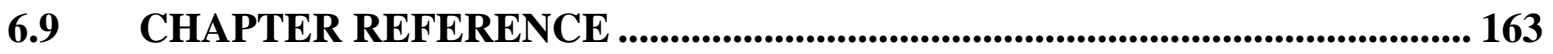

7.0 - IMPROVING STUDENTS' UNDERSTANDING OF THE ADDITION OF

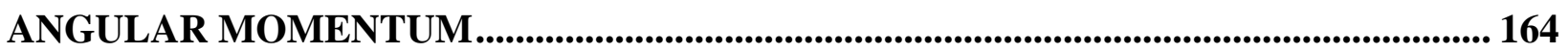

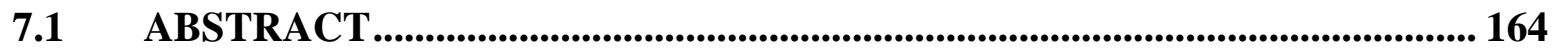

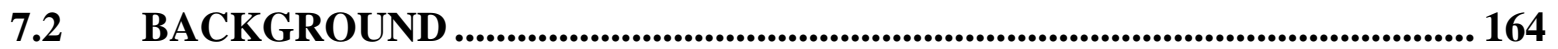

7.3 INVESTIGATION OF STUDENTS’ DIFFICULTIES ........................................ 166

7.3.1 Difficulty with the Dimension of the Hilbert Space ................................ 167

7.3.2 Difficulty in identifying different basis vectors for the product

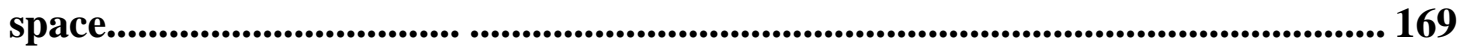

7.3.3 Difficulty in constructing an operator matrix in the product space .172

7.3.4 Difficulty in finding the probabilities for measuring an observable. 175

7.4 QUILT FOR ADDITION OF ANGULAR MOMENTUM ............................... 176

7.4.1 Dimension of Hilbert space ............................................................................. 176 
7.4.2 Constructing matrices for different operators for the product space of two spin-1/2 systems in the uncoupled representation.............................................. 179

7.4.3 Introducing the coupled representation...................................................... 181

7.4.4 Constructing matrices for different operators for the product space of two spin-1/2 systems in the coupled representation..................................................... 183

7.5 PRELIMINARY EVALUATION ........................................................................... 184

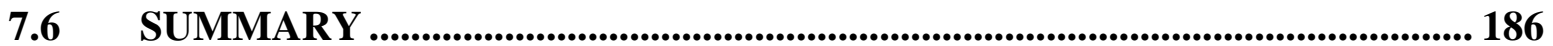

7.7 CHAPTER REFERENCE .................................................................................. 188 8.0 - IMPROVING STUDENTS' UNDERSTANDING OF QUANTUM MECHANICS BY USING PEER INSTRUCTION TOOLS IN CLASS.............................................................. 189

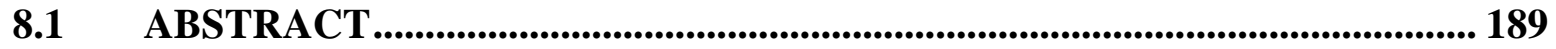

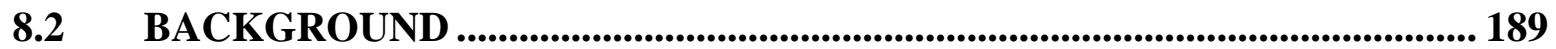

8.3 OVERVIEW OF CONCEPT TESTS ................................................................... 192

8.4 SAMPLE CONCEPT TEST QUESTIONS................................................... 193

8.5 PRELIMINARY EVALUATION ......................................................................... 194

8.6 CONCLUSION ............................................................................................................ 197

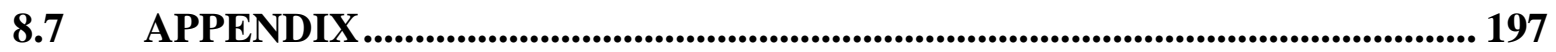

8.8 CHAPTER REFERENCE .................................................................................. 199

9.0 - SURVEYING STUDENTS’ UNDERSTANDING OF QUANTUM MECHANICS . 200

9.1 ABSTRACT ........................................................................................................ 200

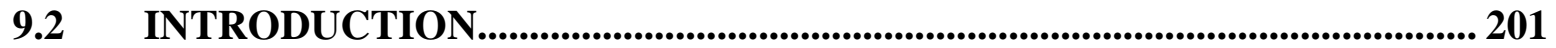

9.3 SURVEY DESIGN................................................................................................ 202

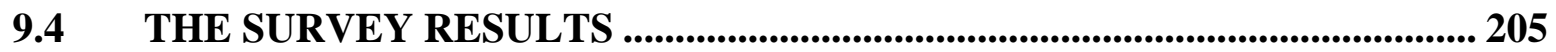


9.5 ITEM ANALYSIS............................................................................................... 207

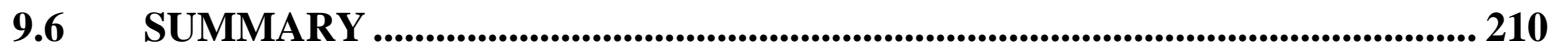

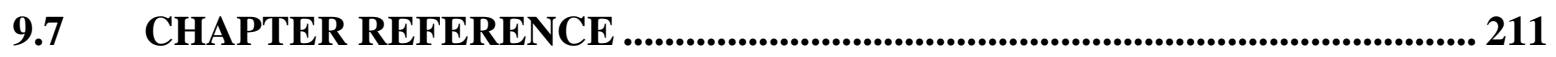

10.0 - CONCLUSIONS AND FUTURE CONSIDERATIONS.............................................. 212

10.1 QUANTUM INTERACTIVE LEARNING TUTORIALS (QUILTS) ............ 212

10.2 CONCEPT TESTS .............................................................................................. 213

10.3 QUANTUM MECHANICS SURVEY (QMS) .................................................. 214

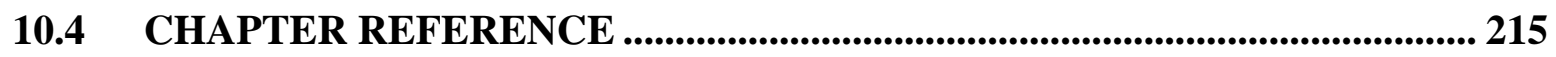

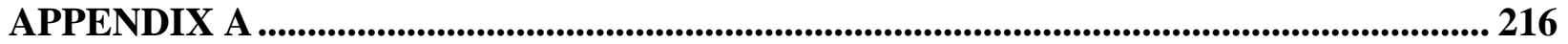

APPENDIX B ................................................................................................................................ 241

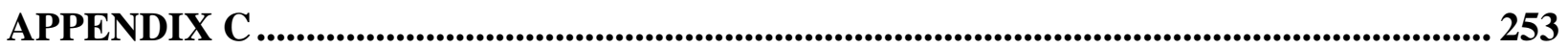




\section{LIST OF TABLES}

Table 2.1 The pre-test and post-test scores on each question of the tests about the possible wavefunctions. 55

Table 3.1 The pre-test and post-test scores on each question of the tests about the bound and scattering state wavefunctions 74

Table 3.2 The control group scores on each question of the tests about the bound and scattering state wavefunctions. 76

Table 5.1 The pre-test and post-test scores on each question of Test A about quantum measurement. 130

Table 5.2 The pre-test and post-test scores on each question of Test B about quantum measurement. 131

Table 6.1 The pre-test and post-test scores on each question of the tests about Stern-Gerlach

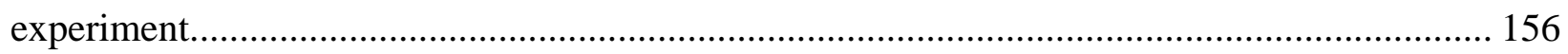

Table 9.1 A possible categorization of the QMS questions................................................. 208 Table 9.2 Students' responses for the two questions about the time dependence of expectation values 208 


\section{LIST OF FIGURES}

Figure 2.1 The slope of the wavefunction is zero in the regions where potential energy is zero. 37

Figure 2.2 A wavefunction localized inside a finite square well............................................ 39

Figure 2.3 Samples of incorrect sketches of wavefunctions which are discontinuous at the

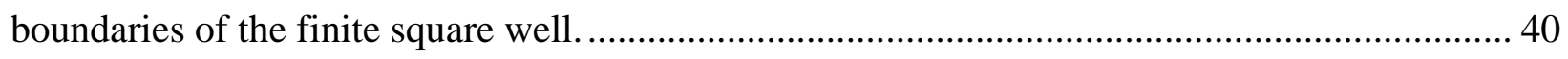

Figure 2.4 A wavefunction with a cusp inside the well is not allowed for an infinite square well.

Figure 2.5 Wavefunction with a cusp inside the well drawn by a student. The wavefunction is not

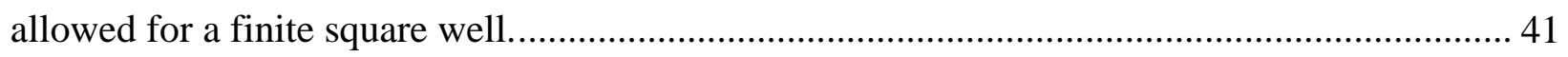

Figure 2.6 A student's sketch to find a graphical solution of the transcendental equation. ........ 43

Figure 2.7 A superposition of stationary state wavefunctions for a 1D infinite square well....... 45

Figure 2.8 OSP simulation of the possible wavefunction in a 1D infinite square well.............. 46

Figure 2.9 The first derivative of the wavefunction is discontinuous at the boundaries so this wavefunction is not a possible wavefunction for a finite square well. .................................. 48

Figure 2.10 A snapshot of the simulation of the finite square well. ........................................ 49

Figure 2.11 The wavefunctions in the classically forbidden region of a finite square well ......... 50

Figure 3.1 An example of classical bound state but quantum mechanical scattering state. ........ 59

Figure 3.2 A potential energy well that does not allow quantum mechanical bound states........ 60 
Figure 3.3 An incorrect sketch of a wavefunction with both bound and scattering states simultaneously. 61

Figure 3.4 A quantum particle in a stationary state cannot have different energies in different regions. 61

Figure 3.5 An example of a bound state particle tunneling into the classical forbidden regions. 62 Figure 3.6 A scattering state particle with energy E lower than the potential energy barrier. ..... 63 Figure 3.7 The particle launched from the left hand side of the potential energy well has a nonzero probability of being bounced back by the potential energy well. 65 Figure 3.8 An energy eigenstate only corresponds to one energy though the potential energy could have different values in different regions. 68

Figure 3.9 A multiple choice question asking students to choose the correct qualitative sketch of a scattering state wavefunction with no directional preference. 70

Figure 3.10 PhET simulation of a scattering state wavefunction incident from the left hand side of a potential energy barrier. 71

Figure 3.11 A particle with energy E interacting with a piecewise continuous potential energy well. 73

Figure 3.12 A particle with energy E incident from the left side of a finite square barrier. 74 Figure 3.13 A student in the control group sketched a "reflected" energy level for the scattering state. 75

Figure 3.14 A student in the control group believed only the energy barrier allows scattering state. 75

Figure 4.1 An incorrect sketch of an energy eigenfunction that oscillates about different axes. . 80 Figure 4.2 Incorrect sketches of an energy eigenfunction in a finite square well. 81 
Figure 4.3 A particle with energy E interacting with a piecewise continuous potential energy well.

Figure 4.4 A particle with energy E $>0$ interacting with a finite square well ........................... 83

Figure 4.5 A particle with energy E interacting with a finite square barrier. ........................... 84

Figure 4.6 A quantum particle interacting with a 1D potential energy well............................. 85

Figure 4.7 A quantum particle interacting with a 1D potential energy barrier.......................... 87

Figure 4.8 A quantum particle interacting with a triangular potential energy well..................... 88

Figure 4.9 A quantum particle interacting with a half SHO potential energy well. ................... 91

Figure 5.1 Pictorial question in the warm-up testing about the energy eigenstates for a 1D infinite square well.

Figure 5.2 Simulation program of the energy measurement on a superposition state with two energy eigenstate components.

Figure 5.3 Simulation program of the energy measurement on a superposition state with ten energy eigenstate components. 115

Figure 5.4 Simulation program of the position measurement on an energy eigenstate. 116

Figure 5.5 Time evolution on a superposition state with two energy eigenstate components.... 122 Figure 5.6 Energy measurement on a superposition state with two energy eigenstate components after time $\mathrm{t}>0$. 123

Figure 5.7 Time evolution of the position eigenstate wavefunction. 124

Figure 5.8 Energy measurement after a position measurement of the initial state with only two energy eigenstate components $n=1$ and $n=2$ 128

Figure 5.9 Consecutive position measurement in quick succession/after waiting for some time 129 
Figure 6.1 Three sample responses in which students provided incorrect explanations for why there should be one spot instead of two in question (2) in the Appendix of Chapter 6 . 141

Figure 6.2 Two sample responses in which students provided incorrect explanations for why the state/beam will bend as shown in response to the magnetic field gradient in question (2) in the Appendix of Chapter 6

Figure 6.3 A diagram drawn by a student showing the Larmor precession of spin in response to question (2) in the Appendix of Chapter 6.

Figure 6.4 Examples of two graduate students' responses to question (9) 146

Figure 6.5 Set up for a guided example in the QuILT. 151

Figure 6.6 A snapshot of the simulated experiment constructed from the OSP SPINS program. 152

Figure 6.7 Analogy between spin states and photon polarization states...................................... 153

Figure 6.8 Pictorial representations used for a SGA............................................................... 159

Figure 6.9 Sketch for question (3) in the Appendix of Chapter 6............................................. 160

Figure 6.10 Sketch for question (4) in the Appendix of Chapter 6............................................. 161

Figure 6.11 Sketch for question (6) in the Appendix of Chapter 6............................................ 162

Figure 6.12 Sketch for question (7) in the Appendix of Chapter 6............................................. 162

Figure 8.1 The distribution of students' individual score of the 1D infinite square well quiz ... 196

Figure 8.2 An electron with energy E interacting with a 1D finite square well......................... 197

Figure 8.3 Potential energies that may allow bound states or scattering states or both............... 198

Figure 9.1 Item Difficulty (fraction correct) for each item on the test for 109 students ............. 206

Figure 9.2 Item Discrimination for each item on the test for 109 students ................................ 206 


\section{PREFACE - ACKNOWLEDGMENT}

I dedicate this thesis to my advisor, Dr. Chandralekha Singh, for all her help and support through the past four years. Without her guidance, I could not finish this degree in physics education and start my academic career in education research.

I would like to thank Dr. Robert Devaty for his great aid and suggestions after reading our research-based tutorials. I would like to thank Dr. Arthur Kosowsky for the valuable discussion about quantum mechanics. I would also like to thank Dr. Rainer Johnsen and Dr. Larry Shuman for their insight and critiques about my research.

In addition, I would like to thank Dr. Jeremy Levy for his insight and suggestions related to our QuILTs and concept tests. I would also like to thank Dr. Ayres Freitas for helping us in conducting the peer instruction materials, QuILTs and QMS in his class. I am also grateful to all the faculty and students who helped with the development and evaluation of our research-based learning tutorials and the standardized survey of quantum mechanics.

I am happy to thank all my family members for their love and support through all these years. Additional thanks are due to my grandparents for taking care of me for many years and to my uncle for the concerns about me in the past years.

I am incredibly grateful to my parents for their endless love and continuous support. Thank you, my dad! And thank you, my mom! I cannot express my gratitude towards you in words. 


\subsection{INTRODUCTION}

Helping students to think like physics experts is an important goal of most physics courses. But learning physics is difficult for students at all levels from the introductory to the advanced. Even in the introductory physics courses, students must draw meaningful inferences from the abstract principles, which are in highly compact mathematical form, and apply the few fundamental principles in diverse situations. Such tasks may be routine for the experts in physics but can be very challenging for the students. A lot of prior research has been conducted on investigating the differences between introductory physics students and physics experts in problem solving, reasoning and meta-cognitive skills (Maloney 1994, Chi et al. 1981, Touger et al. 1995). In general, experts start solving problems at a more abstract level and later turn to the specifics, while the novices may immediately focus on the surface features and get distracted. Experts can apply their knowledge in novel and complex problems depending on the level of their expertise, while novices may only be able to solve familiar problems requiring routine procedures. Experts have knowledge structure which is organized hierarchically, while novices' knowledge structure lacks hierarchical organization.

Novices' difficulties in introductory physics have been widely studied and many instructional strategies have been developed to help introductory physics students acquire the content knowledge as well as the ability to solve problems in novel situations (Leonard et al. 1996, Heller and Reif 1984, Van Heuvelen 1991, Mestre et al. 1993, Dufresne et al. 1992). 
However, it is often assumed that the students in the upper-level physics courses have significantly higher expertise in learning and self-monitoring than those who have only studied introductory physics. Instructors usually take for granted that advanced physics students are independent learners with necessary cognitive and meta-cognitive skills and enough prior knowledge in introductory and intermediate physics. However, advanced students face additional challenges because they must build the upper-level physics knowledge on all of the prior knowledge acquired at the introductory and intermediate levels. Research also suggests that there is a wide diversity in advanced students' skills such as their ability to categorize physics problems based upon similarity of solution or their tendency to exploit their mistakes as an opportunity for repairing and organizing their knowledge structure (Lin \& Singh 2009, Lin \& Singh 2010, Mason \& Singh 2009, Mason \& Singh 2010). Therefore, while teaching upper-level physics courses, treating all the advanced students as a group of experienced learners will not lead to designing of effective instructional strategies and scaffolding support to help them become physics experts.

Indeed, once we are familiar with the prior knowledge of upper-level students, we can consider effective strategies to help them build on their prior knowledge and construct a hierarchical knowledge structure and develop skills in applying relevant knowledge in various situations. An important question is the following: Will the educational methods and techniques that have been effective in introductory physics courses be effective in the upper-level courses as well? In this thesis, I will discuss my research on students' difficulties in learning upper-level undergraduate quantum mechanics and the impact of incorporating tutorial-based instruction and peer-instruction tools in helping students learn better. 
In particular, I will discuss how the findings of cognitive research and Physics Education Research (PER) in introductory physics can guide the investigation of students' difficulties and strategies to help students learn quantum mechanics better. In the remaining paragraphs of chapter 1, I will first provide some motivation for why quantum mechanics is so difficult for the advanced students to learn and then introduce the cognitive issues and educational strategies that take into account the findings of learning theories that can help students learn quantum mechanics better. In chapter 2 to chapter 7, I will summarize and categorize students' difficulties and misconceptions about quantum mechanics related to topics such as "Possible Wavefunctions", "Bound and Scattering State”, "Drawing Wavefunctions", "Quantum Measurement”, “Stern-Gerlach Experiment”, “Addition of Angular Momentum”, etc. Based upon the findings of investigation of students' difficulties, we developed a set of research-based learning tutorials called the Quantum Interactive Learning Tutorials (QuILTs) and peer instruction tools, e.g., concept tests, to scaffold student learning of quantum mechanics and help them construct a hierarchical knowledge structure. The details of the development, implementation and findings of these research-based QuILTs and peer-instruction tools will be elaborated in chapters 2 to 8 . In the ninth chapter, I will discuss the development and preliminary assessment of a standardized survey that can help instructors better probe students' conceptual understanding of quantum mechanics in their classes. Then, conclusions and future directions will be outlined in the final chapter. 


\subsection{QUANTUM MECHANICS VS. CLASSICAL MECHANICS}

Quantum mechanics (QM) is an important topic in the physics curriculum and it is also important for students majoring in other sciences, e.g., chemistry or engineering, e.g., electrical engineering. Students who are interested in the basic rules governing the universe beyond Newtonian physics are often fascinated by quantum mechanics. However, quantum mechanics formalism is abstract and does not conform to the everyday world we are used to in which position and momentum are deterministic variables and their time evolution is governed by Newton's laws. Quantum phenomena cannot be explained in classical ways. Talented students have great difficulty in mastering the fundamental concepts and principles of quantum mechanics. Richard Feynman said “nobody understands quantum mechanics” (Feynman, 1965). Feynman’s statement was referring to the difficulty in interpreting the foundational issues in quantum mechanics rather than the difficulty in performing a calculation based upon quantum mechanics formalism. However, it is important to research effective strategies to help students learn the standard formalism of quantum mechanics.

As noted earlier, unlike classical mechanics, we do not have direct experience with the microscopic quantum world. Also, quantum mechanics has an abstract theoretical framework in which the most fundamental equation, the Time-Dependent Schrödinger Equation (TDSE), describes the time evolution of the wave function or the state of a quantum system according to the Hamiltonian of the system. This wave function is in general complex and does not directly represent a physical entity. However, the wave function at a given time can be exploited to make inferences about the probability of measuring different physical observables associated with the system. For example, the absolute square of the wave function in position-space is the probability density for position measurement. Since the TDSE does not describe the evolution or 
motion of a physical entity, unlike Newton's second law, the modeling of the microscopic world in quantum mechanics is generally more abstract than the modeling of the macroscopic world in classical mechanics.

Quantum theory provides a coherent framework for reasoning about microscopic phenomena and has never failed to explain observations if the Hamiltonian of the system is modeled appropriately to account for the essential interactions. However, the conceptual framework of quantum mechanics is often counter-intuitive to our everyday experiences. For example, according to the quantum theory, the position, momentum, energy and other observables for a quantum mechanical entity are in general not well-defined. We can only predict the probability of measuring different values based upon the wave function when a measurement is performed. This probabilistic interpretation of quantum mechanics, which even Einstein found disconcerting, is challenging for students. Moreover, according to the Copenhagen interpretation of quantum mechanics, which is widely taught to students, the measurement of a physical observable changes the wave function if the initial wave function is not an eigenfunction of the operator corresponding to the observable measured. Thus, the usual time evolution of the system according to the TDSE is separated from what happens during the measurement of an observable. Students often have difficulty with this notion of an instantaneous change or "collapse" of the wave function during the measurement.

In quantum theory, position and momentum are not independent variables that evolve in a deterministic manner but are operators in the Hilbert space in which the state of the system is a vector. For a given state of the system, the probabilities of measuring position or momentum in a narrow range depend on each other. In particular, specifying the position-space wave function that can help us determine the probability of measuring the position in a narrow range specifies 
(via a Fourier transform) the momentum-space wave function that tells us the probability of measuring the momentum in a narrow range. The eigenstates of the position or momentum operators span the Hilbert space so that any state of the system can be written as a linear combination of a complete set of position eigenstates or momentum eigenstates. The measurement of position (or momentum) yields a position (or momentum) eigenvalue with a certain probability depending upon the state of the system. These concepts are indeed challenging for students since they do not conform to the experiences in the classical world (Singh 2007).

In addition to the lack of direct exposure to microscopic phenomena described by quantum theory and the counter-intuitive nature of the theory, the mathematical facility required in quantum mechanics can increase students' cognitive load and make learning quantum mechanics even more challenging. The framework of quantum mechanics is based on linear algebra. In addition, a good grasp of differential equations, special functions, complex variables, etc., is highly desired. If students are not facile in relevant mathematics, they may become overwhelmed by the mathematical details and may not have the opportunity to focus on the conceptual framework of quantum mechanics and build a coherent knowledge structure. Earlier research (Singh 2007) shows that a lack of mathematical facility can hinder conceptual learning. Similarly, alternative conceptions about conceptual aspects of quantum mechanics can lead to students making mathematical errors that they would otherwise not make in a linear algebra course (Singh 2007).

Many of the alternative conceptions in the classical world are over-generalizations of everyday experiences to contexts where they are not applicable. For example, the conception that motion implies force often originates from the fact that one must initially apply a force to an 
object at rest to get it moving. People naively over-generalize such experiences to conclude that even an object moving at a constant velocity must have a net force acting on it. One may argue that quantum mechanics may have an advantage here because the microscopic world does not directly deal with observable phenomena in every day experience so students are unlikely to have alternative conceptions. Unfortunately, that is not true and research shows that students have many alternative conceptions about quantum physics (in the level of modern physics) and quantum mechanics (McKagan et al. 2008(a), McKagan et al. 2008(b), McKagan et al. 2008(c), McKagan et al. 2009, Jolly et al. 1998, Singh et al. 2006, Wittmann et al. 2002, Zollman et al. 2002, Styer 1996, Johnston et al. 1998, Ireson 2000, Bao and Redish 2002, Carr and McKagan 2009, Fischler et al. 1992, Redish et al. 2001, and the theme issue of American Journal of Physics 2002). These alternative conceptions are often about the quantum mechanical model itself and about exploiting this model to infer what should happen in a given situation. Students often over-generalize their intuitive notions from the classical world to the quantum world, which can lead to incorrect inferences.

\subsection{A SHORT REVIEW OF RELEVANT PHYSICS EDUCATION RESEARCH (PER)}

Physics education research is discipline-based research conducted mostly by physicists with knowledge of physics and access to students. It became an established field of research in the physics departments in the United States in the late twentieth century. In the 1980s through the 1990s, the famous test to assess students' conceptual understanding of introductory physics, the

Force Concept Inventory (FCI) (Hestenes et al. 1992; Hestenes \& Halloun 1995), made physicists realize that despite their sincerest effort, solely teaching via lectures is not effective in 
helping students learn physics. They found that their students lacked conceptual understanding of physics even though they could solve complicated quantitative problems using a rote algorithmic approach. The number of physicists doing PER and developing and assessing research-based instructional strategies has grown steadily over the last few decades. In 1999, the American Physics Society (APS) published the "Statement on Research in Physics Education", which announced the usefulness and the validity of PER in physics departments (Beichner, 2009). In 2005, the Physical Review series welcomed a new journal, Physical Review Special TopicsPhysics Education Research, indicating that PER formally became an essential part of scientific research in physics.

Physics education research generally focuses on two areas, the basic PER and the applied PER (Beichner, 2009). The basic PER concerns the assessment and determination of students' difficulties in understanding physics concepts and the applied PER focuses on developing effective teaching strategies or instructional materials to help students overcome their common difficulties and build a robust knowledge structure of physics. My research on improving students' understanding of quantum mechanics involves both basic and applied PER. We have conducted in-depth research on students' difficulties and revealed not only the misconceptions that students have but also unpacked how these misconceptions originate. Based on our research on students' common difficulties in learning quantum mechanics, we have designed the Quantum Interactive Learning Tutorials (QuILTs) and peer-instruction tools such as concept tests to improve students' understanding. 


\subsection{COGNITIVE ISSUES IN PER}

PER has an active interaction with cognitive science. PER uses the theory of cognitive science as an important source for interpreting student learning of physics. Cognitive principles are also useful for the PER researchers in developing and assessing instructional strategies. On the other hand, PER adds to the cognitive research because cognition issues such as metacognition or the nature of expertise can be researched via PER since physics is a domain in which expertise can be assessed more readily than in the social sciences or humanities. While investigating students' difficulties in learning quantum mechanics and developing research-based learning tools, we took into account findings of cognitive research such as those related to memory, metacognition and epistemology, in order to interpret students' reasoning processes and learning outcomes after interventions with QuILTs and peer-instruction tools. Our research is informed by the research of many cognitive and social scientists. In developing research-based learning tools, several cognitive theories and models are carefully integrated, e.g., Piaget's “optimal mismatch”, Vygotsky’s “zone of proximal development”, and the Preparation for Future Learning model of Bransford and Schwartz which is based upon the notions of “efficiency vs. innovation” (Smith 1985, Piaget 1964, Raymond 2000, Bransford \& Schwartz 1999, Schwartz et al. 2005). Below, I

review some of the relevant concepts from cognitive science that have helped inform my research.

\subsubsection{Memory}

In cognitive science, memory refers to the brain's ability to store, retain and retrieve information. Human memory consists of two major components: short-term memory (or working memory) 
and long-term memory (Simon, 1974). In his famous paper about "the magic number seven, plus or minus two", Miller claimed that the storage ability of short-term memory is limited to 5 to 9 bits, where one bit of information is defined as "the amount of information that we need to make a decision between two equally likely alternatives” (Miller, 1956). Due to the limitation of shortterm memory, people cannot process many disparate bits of information at the same time. The short-term memory processes the information for a period of around 18 seconds without repetitive practice and rehearsal (Peterson and Peterson, 1959). The long-term memory is where the information is stored and this information can last from a few days to even a life-time. The capacity of long-term memory can be considered as unlimited unlike the short term memory which is used to process the information. Information processing and problem solving happens in short term memory or working memory which receives information from the sensory buffers (e.g., ears, eyes, hands) and also from the long term memory.

Later research shows that the capacity of the short-term memory can be increased by chunking the information into meaningful groups. For example, a ten-digit number is difficult to remember. But people can often memorize a phone number by dividing the string into three " 3 digits -3 digits -4 digits” chunks so that each chunk of digits has a specific association (e.g., the area code). Research has also shown that expertise in a particular domain involves having large chunks of knowledge in the domain in which the person has expertise. In a study involving positions of chess pieces in a good game of chess (Chase \& Simon, 1973), the chess masters could reproduce the setup of a chess game faster and more accurately than novices because they could chunk the relative positions of the pieces into offensive and defensive patterns when observing a good game board. However, these same experts in chess showed no more advantage over the novice if the setup was just a random positioning of the pieces on the chess board. These 
findings are relevant for my research because it is important to investigate the prior knowledge of students and help them build on this prior knowledge and assist them in chunking relevant knowledge during the learning process.

Information stored in the long-term memory can be retained longer and retrieved faster by practicing and creating associations. People forget what they learned at a rate which is exponential (known as the Ebbinghaus forgetting curve). Research shows that spaced practice and manipulation of repetition time are effective for retaining and retrieving knowledge (Landuaer \& Bjork 1978, Melton 1970). Therefore, in the research-based learning tools I have developed for QM, the questions about key concepts occur not only in the sections in which they are introduced but also in the later sections to provide spaced practice.

While solving a problem, after receiving information from the sensory buffers, the shortterm memory searches for the relevant knowledge in the long-term memory. Developing associations between different concepts and principles and building a robust knowledge structure provide additional links and pathways to activate relevant information during the problem solving process. For example, the momentum operator $\hat{p}$ in one dimension can be represented by the derivative $-i \hbar \partial / \partial x$ in the position space, and the Hamiltonian operator of a system can be written as $\hat{H}=-\left(\hbar^{2} / 2 m\right) \partial^{2} / \partial x^{2}+V(x)$. If a student knows that the Hamiltonian operator corresponds to the total energy of the system, which equals the kinetic energy plus the potential energy, then he/she only needs to remember the representation of the momentum operator and the definition of the Hamiltonian. The mathematical representation of the Hamiltonian can be constructed with this knowledge. Association of knowledge also helps people to better chunk the information and often this chunking happens subconsciously. In introductory physics, the symbols $v$ and $m$ are often associated with the concepts of speed and mass, which are also 
associated with the concept of momentum. While a novice in physics may treat speed, mass, velocity and momentum as four different bits and they may take four slots in the short term memory, for an expert physicist all these related concepts may be chunked and may only take one slot in the short terms memory. Thus, an expert can use his/her “compiled knowledge” and only one bit to process information about momentum without realizing that he/she has already processed a lot of related concepts. While developing learning tools for Quantum Mechanics, we always do a theoretical task analysis which amounts to making a fine-grained flow chart of all the relevant concepts that need to be invoked to solve the problem. Then we analyze the difficulty of the concepts from students' perspective because the difficulty of a problem not only depends on its inherent complexity but on the familiarity and intuition one has developed about it.

\subsubsection{Metacognition \& Epistemology}

Metacognition is the "cognition of cognition" or "knowing about knowing” as referred in Metcalfe \& Shimamura’s book (Metcalfe \& Shimamura 1994). For example, people are engaged in metacognition if they discern that they have more difficulties in learning one concept than another or if they decide to re-examine some information before they accept it as a fact (Flavell, 1976 p.232). Development of metacognitive skills such as reflection and self-awareness in the problem solving process must be addressed while students are learning physics content. Several researchers have investigated the factors that can influence the development of reasoning and metacognitive skills (Yerushalmi \& Eylon 2003, Scott et al. 2007). Moving beyond an algorithmic plug and chug approach and focusing on conceptual understanding can help students develop metacognitive skills (Leonard et al. 1996). In the research-based learning tools we have developed for quantum mechanics, e.g., to help students learn about quantum measurement, and 
to help them sketch a possible bound or scattering state wave function for a given potential energy, we emphasize the development of conceptual reasoning and metacognitive skills and help students focus on their knowledge structure.

Epistemology is the theory of knowledge which attempts to make sense of the human intellectual achievement (Cruz 2006). Research has shown that students’ epistemological beliefs about physics can significantly affect what students learn (Hammer 2000; Schommer 1990, Redish et al. 1998, Adams et al. 2006, Gray et al. 2008). If students believe that physics is a collection of isolated formulas and facts, they will be reluctant to take the time to hierarchically organize their knowledge structure. Likewise, if students believe that their task in a physics class is to take notes, memorize facts and do plug and chug in the exams, they will make little effort to synthesize the content, build connections between new and prior knowledge, extend their knowledge to new areas and contemplate how principles of physics explain physical phenomena. It is indeed impossible for a student without a productive epistemology about the knowledge of physics to become an expert in physics. The research-based instructional tools I have developed for quantum mechanics keep students actively engaged in the learning process and force them to pay attention to the structure of knowledge in quantum mechanics. The learning tools help students realize that despite the abstractness of the subject matter, quantum mechanics is not a collection of incoherent facts and formulas. Students can also learn about how quantum mechanics can be applied to accomplish novel tasks that cannot be accomplished by classical means, e.g., to send a secret key for encoding and decoding data securely over a public channel. 


\subsubsection{Optimal Mismatch.}

Instructors can pose tasks to students in which common difficulties and misconceptions are elicited and then students observe something that contradicts their initial prediction (Smith, 1985). Piaget emphasized "optimal mismatch" between what the student knows and where the instruction should be targeted in order for desired assimilation and accommodation of knowledge to occur. Piaget’s notion of “optimal mismatch” argues that when students encounter a cognitive conflict since their predictions and observations do not match, they are in a state of disequilibrium and they realize that there is some inconsistency in their reasoning (Piaget 1964, p. 29). In this state, students are generally eager to resolve the discrepancies between their prediction and observation. Piaget suggested that at this point students should be provided with appropriate guidance and support commensurate with their prior knowledge to resolve the discrepancies and assimilate and accommodate appropriate concepts.

Not only should students be helped to understand why the relevant concepts are applicable but also why their initial reasoning was not appropriate in that context. When learning quantum mechanics with the research-based learning tools, students are often asked to predict what should happen in different situations and then they use visualization tools such as computer simulations or graphical demonstrations to examine what actually happens. If their predictions are inconsistent with their observation, they are in a state of disequilibrium. Then, the learning tools provide scaffolding to help them resolve the discrepancies and help them build a robust knowledge structure. 


\subsubsection{Zone of Proximal Development (ZPD)}

The concept of the zone of proximal development (ZPD) was originally developed by Vygotsky in the early twentieth century. The definition of ZPD is commonly accepted as "the distance between what children can do by themselves and the learning that they can be helped to achieve with competent assistance” (Raymond 2000 p.176). Thus, Vygotsky's notion of the "zone of proximal development” refers to what a student can do on his/her own vs. with the help of an instructor who is familiar with his/her prior knowledge and skills. Scaffolding is at the heart of ZPD and can be used to stretch a student's learning far beyond his/her initial knowledge by carefully crafted instruction which is designed to ensure that the student makes desired progress and gradually develops independence. With awareness of students' initial knowledge state, the instructor can continuously target instruction a little bit above students' current knowledge state to ensure that the students have the opportunity and ability to connect new knowledge with what they already know and build a robust knowledge structure.

Similar to the optimal mismatch theory, the ZPD theory emphasizes the importance of building students' knowledge structure based on their prior knowledge. Teachers need to provide scaffolding support to stretch students' learning process and help them overcome the gaps between their current knowledge and the new knowledge they are expected to acquire.

Since all students in the advanced courses such as quantum mechanics may not have the same preparation and prior knowledge, it is important to align the learning tools to meet the needs of a diverse group of students. To prepare everyone for the QuILTs, we have designed warm-up materials that students can do at their own pace at home. The warm-up helps students review the necessary preliminary knowledge before they start using the QuILT involving the 
quantum concepts recently learned. For example, in the QuILT related to the Stern-Gerlach experiment, the warm-up material asks the students to consider the basic concepts such as the Hamiltonian of a particle with a magnetic dipole moment in an external magnetic field, the forces acting on the magnetic dipole moment in a magnetic field in a classical situation, the matrix representation of the angular momentum, etc. By working on the warm-up materials, students are likely to have similar prior knowledge before working on the QuILTs.

As stated in chapter 1.3.1, there are only 5 to 9 bits in one's short term memory (or working memory) but the size of the "bit” or chunk can be different depending upon a person’s expertise in that domain. Therefore, it is important to be familiar with students' prior knowledge and have an understanding of what constitutes a bit for them so that they do not have a cognitive overload. One strategy to reduce the cognitive load is having students work with each other because according to the theory of distributed cognition, the cognitive load it shared between individuals working together. In other words, combined working memory is available for problem solving and learning. The instructional method involving peer learning that can scaffold student learning will be elaborated in the section on peer instruction (chapter 1.5).

\subsubsection{Preparation for Future Learning}

In the 1990s, Bransford and Schwartz carried out a series of research on transfer of learning from one situation to another (Bransford \& Schwartz 1999) and proposed a framework for scaffolding student learning. They theorized that the preparation for future learning (PFL) and transfer of knowledge from the situation in which it was acquired to new situations are optimal if instruction includes both the elements of innovation and efficiency. In their model, efficiency and innovation are two orthogonal coordinates. If instruction only focuses on efficiency, the 
cognitive engagement and processing by the students will be diminished and they will not develop the ability to transfer the acquired knowledge to new situations. Similarly, if the instruction is solely focused on innovation, students may struggle to connect what they are learning with their prior knowledge so that learning and transfer will be inhibited. They propose that the preparation for future learning and transfer will be enhanced if the instruction focuses on moving along a diagonal trajectory in the two dimensional space of innovation and efficiency.

Out of the two essential parameters for the transfer of learning, one way to define "efficiency" is the ability to "rapidly retrieve and accurately apply appropriate knowledge and skills to solve a problem with understanding and explanation” (Schwartz et al. 2005). Generally speaking, the best method for increasing efficiency is, as the aphorism says, "practice makes perfect”. Anderson's research on the effect of practice suggests that information can be retrieved faster from the long term memory while solving problems through more practice (Anderson 1999). More practice in applying the same knowledge to different contexts also enhances people's ability to break down a new task into several routine problems which can be easily solved (Schwartz et al. 2005).

However, over-emphasis on efficiency in the transfer of knowledge has the serious disadvantage of producing “functionally fixed behaviors” (Luchins 1942) or "routine experts" who can quickly and accurately solve the familiar problems but are not be able to go beyond the routine procedures (Hatano \& Inagaki 1986, Hatano \& Oura 2003). People focusing on efficiency can be confined in their own routine task without stepping out to analyze the problems from a different angle. One interesting example is the story told by James Adams in the book “Conceptual blockbusting: A guide to better ideas” (Adams 1990). He mentions that a group of mechanical engineers were struggling to design a machine which could pick up tomatoes without 
bruising them. Though these engineers tried many ways to improve the tomato picker, no inspiring outcome occurred. Then some botanists joined this project and provided a different viewpoint—cultivate stronger tomatos with thicker skin! Similarly, when we interviewed students in traditionally taught quantum mechanics courses about how the wave function of the system would evolve after a position measurement, a majority of students' incorrect responses can be classified in two categories: some of them claimed that the system will be stuck in the position eigenstate while others claimed that it will go back to the initial state. Even if we told students that neither of these choices were correct, they could not think of another option, e.g., the wavefunction will evolve with time according to the Time-dependent Schrödinger equation (which is the correct answer). Instead, many students did not believe that there can be another choice. They would often argue with statements such as the following "if the system neither stays in the collapsed state nor goes back to the initial state, where could it go"?

Therefore, for robust transfer of learning, instructional tools should include elements of "innovation". Innovation sometimes originates from the stages of disequilibrium when people find that their routine ways of thinking does not work (Schwartz et al. 2005). Creating optimal mismatch opportunities via innovative learning tools can be helpful in not only building a knowledge structure but also in enhancing students' innovative ability. The advanced students in quantum mechanics often have a reasonable expertise in introductory physics and classical mechanics but they are "novices" in quantum mechanics. Effective instructional tools can help students go beyond their routine reasoning processes in classical mechanics and learn to think in a quantum mechanical way.

In physics courses, straight lectures are often used as an efficient strategy for conveying knowledge. However, if students are not given an opportunity to think, they may memorize the 
algorithms and definitions of concepts without developing a functional understanding of the fundamental principles of physics. On the other hand, if students are given innovative tasks that force them to think about the physics principles involved beyond what they have been told, they may be able to interpret the concepts better and build a good knowledge structure. But if these innovative tasks are too challenging and beyond students' zone of proximal development, students can get frustrated, may not pursue the task as desired and hence may not learn. Thus, a balance of efficiency and innovation is required for learning to be meaningful and for appropriate transfer of knowledge to occur (Schwartz et al. 2005). The pace of efficient instruction and the complexity of the innovative tasks should therefore be carefully controlled (Schwartz et al. 2005). By considering the issues related to innovation and efficiency together in an educational process, learners can become "adaptive experts" who are not only able to solve routine problems but can also utilize their knowledge to solve novel problems in a new domain (Hatano \& Inagaki 1986).

We note that one common element of all of these seemingly different frameworks discussed in this and the previous two sections is their focus on students' prior knowledge in order to scaffold learning. Indeed, instructional tools must be designed with students' prior knowledge in mind in order for instruction to be in the zone of proximal development and to provide optimal mismatch to ensure adequate preparation for future learning. 


\subsection{GUIDED INQUIRY APPROACH}

Traditionally, physics instruction is based on the instructors' perspective of the course materials and the instructors' anticipation of the students' level (McDermott 1991). Instructors usually have expertise in physics (at least instructors at the college level). Unfortunately, without guidance and support related to effective teaching, many instructors have difficulty understanding the importance of knowing the prior knowledge of students for effective teaching. They have difficulty putting themselves in students' shoes and often consider the difficulty of the subject matter from their perspective instead of the students' perspective. Often, instructors do not use a systematic approach to problem solving which includes performing a conceptual analysis, planning and decision making before the implementation of the plan. Moreover, the instructor may automatically use problem solving as an opportunity for repairing, extending and organizing their knowledge, but reflection and metacognition must be taught explicitly to students. They themselves reflect upon why a principle of physics was suitable in one situation but not in another situation and how they will recognize the next time that principle is relevant in other situations. Without explicit guidance, students may not understand the importance of reflection, metacognition and knowledge organization in mastering physics. For example, most students in an introductory physics course know the statement of Newton's third law that the action and reaction forces of two bodies are equal in magnitude and opposite in direction. However, many students still believe that a heavy truck exerts more force on a small car when they crash. While students may be given some quantitative problem asking them to find the accelerations of the truck and the car after collision, they may look at a solved example problem and obtain an answer to the quantitative question asked without internalizing that the forces on

the truck and car are equal in magnitude. As long as they can get an answer to a quantitative 
problem, students often do not go through the deeper reasoning process to build the connections between the new knowledge and their previous experiences, reconcile the differences, and repair and build a robust knowledge structure.

Research shows that students must be actively engaged in the learning process for learning to be meaningful. To overcome the disadvantages of traditional instruction, inquirybased teaching and learning strategy has been introduced in science education. In 1996, the National Science Education Standards asserted that the study of science "must emphasize student understanding through inquiry” (National Research Council 1996 p.212). In the National Science Education Standards, inquiry is defined as follows (National Research Council 1996, p.23):

"Scientific inquiry refers to the diverse ways in which scientists study the natural world and propose explanations based on evidence derived from their work. Inquiry also refers to the activities of students in which they develop knowledge and understanding of scientific ideas, as well as an understanding of how scientists study the natural world.”

Guided inquiry is a commonly used technique in an inquiry-based instruction. In the guided inquiry approach, the instructor provides the course materials and appropriate "guiding" questions for the students to investigate (Colburn 2000). The guided inquiry approach reflects how people understand the world and how the scientific knowledge is developed. Thus, it is a more natural way for the students to construct their knowledge structure with guidance from the questions that students are asked to investigate. In the learning cycle of a guided inquiry approach, students first work on the questions in the learning materials using their prior knowledge so that they can develop their own explanations based upon their current understanding of relevant scientific concepts and principles. Then, they can discuss their reasoning and explanations with their classmates to make sure that their interpretations are 
consistent with others. The students can also be posed questions in other situations and asked to evaluate whether their reasoning is consistent with what actually happens in those situations and with the guidance and perspective provided by the instructor. If the students find that their reasoning is inconsistent with the perspective provided, they can examine possible misconceptions and gaps in their knowledge. After the students reconcile the differences between their initial reasoning and the correct perspective, another question can be posed to guide them to investigate a new aspect of the concepts they just learned and to help them build a robust knowledge structure.

Some have argued that inquiry-based learning provides "minimal guidance” so it cannot be more effective than the traditional lecture or direct instruction (Kirschner et al. 2006). However, further research clarifies the difference between inquiry-based instruction and a "minimal guidance" approach in which students have very little guidance and shows evidence of the effectiveness of guided-inquiry approach in the learning process (Hmelo-Silver et al. 2007). In fact, the guided-inquiry approach provides extensive scaffolding and rich guidance for students learning scientific principles. When the questions used in the guided-inquiry approach are carefully designed, it can scaffold student learning and help them build a robust knowledge structure. Moreover, the role of the instructor in an inquiry-based class is not simply that of a person who supplies a set of questions that build on each other. He/she must pay attention to the students' reasoning process and monitor students' learning. The instructor can also respond to students' questions and guide them appropriately.

We note that inquiry-based instruction does not exclude other teaching and learning strategies. As noted in the National Science Education Standards, instructors should use different approaches to develop students' knowledge and scientific abilities (National Research Council, 
1996, p23). Traditional lecture still has its advantage in efficiently distributing the necessary information, especially when the class time is not long enough to cover everything using an inquiry-based approach. Therefore, when developing research-based learning tools for quantum mechanics, we make the guided-inquiry approach compatible with the traditional lectures. For example, the instructors can prepare their lectures as they used to but add QuILTs and concept tests as inquiry-based learning tools during the lecture to help students develop a good grasp of physics concepts.

For example, to help students develop a better understanding of quantum mechanics via the Stern-Gerlach experiment or to help with their understanding of issues related to quantum measurement, we have designed the corresponding QuILTs which use a guided-inquiry approach based on students' common difficulties and prior knowledge. Each QuILT typically contains 20 to 30 guided questions. In a QuILT, we may use a group of 3 to 5 questions to address one aspect of the new knowledge. At the end of each group of questions, necessary feedback is provided to students via computer simulations, illustrations and a general class discussion of the issues. If the QuILT is implemented in class, the instructor can lead a discussion at the end of each group of questions to ensure that everybody benefits from what others have learned. The difficulty level of the questions as well as the connection between different groups of questions in a QuILT is carefully monitored. A QuILT can also be used as a homework supplement or as a self-study tool by students. 


\subsection{PEER INSTRUCTION}

Peer instruction was popularized by Mazur at Harvard University in the 1990s. As stated in Mazur's manual of peer instruction, the fundamental goal of implementing peer instruction strategy in class is "to exploit student interaction during lectures and focus students' attention on underlying concepts” (Mazur 1997 p.10). This statement actually points out two commonly existing problems in many physics classrooms. One problem is that students have little interaction with the instructor and their classmates so they have inadequate opportunity to benefit from such interactions and reflect on what they are taught. Most students just sit in the classroom and copy everything on the blackboard or powerpoint slides. Or in some cases, students would not bother to come to the class if they can download the slides online or copy the lecture notes from their classmates. Students are often too busy in taking notes to ask a question or discuss their confusions with the instructor and classmates. Then after the class, they are very likely to forget about their questions. Some professors ask informal questions in the class to interact with the students. However, usually only a small group of students in the class are willing to answer the questions and the silent majority in the class do not get involved.

The other common problem in the traditional physics classes is that students pay less attention to the qualitative interpretation than the quantitative skills when learning physics. Students only learn what they are tested on. Since most of the questions in the homework and exams in a traditional physics course ask the students to calculate a physical quantity or derive an equation, things that can be done algorithmically, so that students often have the epistemological misunderstanding that physics is just a collection of formulas and algorithms. Without incentive, students make little effort to interpret the concepts and principles and learn to organize their knowledge hierarchically. They tend to use a plug and chug approach to solving physics 
problems by looking for a suitable formula in which they could plug in all the variables given in a problem statement. But algorithmic quantitative exercises cannot automatically improve students' conceptual understanding. Research has shown that high-performing students on quantitative tests may fall in the low-performing group on conceptual tests (Mazur 1997 p.7-10). Therefore, it is of great importance to help students develop conceptual understanding and build a robust knowledge structure of physics.

In the peer-instruction approach, concept tests are used as a guidance to lead peer discussions in class. A concept test question is usually a multiple-choice question related to a core concept or principle that is being discussed in the course. Most of the time, the options in each multiple-choice question have been prepared before the lecture (with alternative choices often dealing with common difficulties) though in some cases the instructor can ask the students to provide the possible answers and then let the class vote on these ideas. For a class using the peer instruction method, the class hour can be divided into several pieces of presentations focusing on each central point (Crouch \& Mazur 2001). At the end of each short presentation, the corresponding concept test questions are given to the class. Students discuss with a partner the answers to the concept test questions and then they are polled either by electronic clickers (Beatty et al. 2006), show of cards (with A through E written on each card) or by show of hands for each choice in the multiple-choice question.

There are tremendous advantages to implementing peer instruction in class. First of all, students are actively engaged in the learning process instead of passively listening to the lecture and taking notes. During the peer discussion, students must convey their understanding about the relevant concepts to their peers as well as examine their peers' interpretation and reasoning. Since this task is challenging, students must constantly be on their toes. They must focus on what 
they just learned, discussing with their peers, and repair, extend and organize their knowledge in their long term memory.

Secondly, in the peer instruction approach, students are encouraged to ask questions. Moreover, when a student gives an answer after a group discussion and the answer turns out to be incorrect, the student would treat it as "our" mistake instead of "my" mistake. Thus, he/she is less shy or awkward to ask for clarification. In addition, students can often understand each other's difficulty much better than the instructor can because all students have learned the material recently and have gone through similar processes of reasoning and clarification of confusion. Since there is a large gap between the novice and the expert knowledge structure, some mistakes made by the students may seem puzzling to the instructor. On the other hand, students in the class may have experienced and overcome similar difficulty in their reasoning process so they can effectively guide their classmates to the right track. Research have shown that students can "co-construct knowledge" when they are solving problems with peers (Singh 2005). Co-construction of knowledge occurs when neither of the two students in a discussion group could solve the problem individually, but they are able to solve the problem together.

Thirdly, the instructor could have an instant feedback on how well the class understands the content just taught. This feedback can be easily and quickly obtained quantitatively and carefully analyzed later if the classroom is equipped with an electronic clicker system. Students use the remote answering device (clicker) to record their answers for a concept test question and the distribution of their selections can be shown anonymously to the whole class via computer projection. If most students make the right choice, then the instructor can safely move to the next topic. Otherwise, further discussions can take place to make sure that most students in the class have a correct understanding of the relevant concepts. Even if the electronic devices are not 
available in the classroom, the peer instruction method can still be used by asking students to show their answers using cards or a show of hands. The instructor could prepare cards, or simply letter size papers, in different colors with the choices A, B, C, D \& E so that the answer distribution can also be efficiently estimated. The drawback of showing cards or raising hands is the lack of anonymity. In these cases, students’ answers may be influenced by others’ responses.

Last but not least, peer instruction with an electronic clicker system can increase the class attendance which may have positive implications for learning. Points can be awarded to students for tying to answer the clicker questions even if their selections are incorrect. Since students have learned the material recently, students can be awarded most of the points (e.g., 80\%) for trying to answer the clicker questions even if they are not correct. Also instructors should make students realize that questions similar to the concept test questions will be asked in their midterm and final exams so that they take learning with peers seriously instead of randomly pressing a button on the clicker.

\subsection{CHAPTER REFERENCE}

Adams, J. (1990). Conceptual blockbusting: A guide to better ideas. Cambridge, MA: Perseus Publishing.

Adams, W., Perkins, K., Podolefsky, N., Dubson, M., Finkelstein, N., and Wieman, C. (2006). "New instrument for measuring student beliefs about physics and learning physics: The Colorado Learning Attitudes about Science Survey." Physical Review Special Topics Physics Education Research, 2(010101).

American Journal of Physics. (2002). The Theme Issue of American Journal of Physics, 70(3), March 2002

Anderson, J. (1999). Learning and memory: An integrative approach. New York: Wiley. 
Bao, L. and Redish, E. (2002). "Understanding probabilistic interpretations of physical systems: A prerequisite to learning quantum physics." Am. J. Phys., 70(3), 210-217.

Beatty, I., Gerace, W., Leonard, W., and Dufresne, R. (2006). "Designing effective questions for classroom response system teaching." Am. J. Phys., 74(1), 31-39.

Beichner, R. J. (2009). An Introduction to Physics Education Research. http://www.percentral.org/document/ServeFile.cfm?ID=8806\&DocID=1147.

Bransford, J., and Schwartz, D. (1999). "Rethinking transfer: A simple proposal with multiple implications", A. Iran-Nejad and P. Pearson, (eds.), Review of Research in Education. Washington, D.C.: American Educational Research Association, pp. 61-100.

Carr, L. D. and McKagan, S. B. (2009). "Graduate quantum mechanics reform." Am. J. Phys., 77(4), 308-319.

Chase, W., and Simon, H. (1973). "Perception in chess." Cognitive Psychology, 4, 55-81.

Chi, M., Feltovich, P., and Glaser, R. (1981). "Categorization and representation of physics knowledge by experts and novices." Cognitive Science, 5, 121-152.

Colburn, A. (2000). "An Inquiry Primer." Science Scope, 23(6), 42-44.

Crouch, C., and Mazur, E. (2001). "Peer Instruction: Ten years of experience and results." Am. J. Phys., 69(9), 970-977.

Cruz, J. (2006). “Epistemology”. In Encyclopedia of Cognitive Science, edited by Nadal, L., NY: Wiley, published online 15 Jan 2006.

Dufresne, R., Gerace, W., Hardiman, P., and Mestre, J. (1992). " Constraining novices to perform expert-like problem analyses: Effects on schema acquisition." Journal of the Learning Sciences, 2(3), 307-331.

Feynman, R. (1965). The Character of Physical Law, chapter 6. London, United Kingdom: British Broadcasting Corp.

Flavell, J. H. (1976). "Metacognitive aspects of problem solving", L. Resnick, (ed.) The nature of intelligence. Hillsdale, NJ: Erlbaum.

Gray, K., Adams, W., Wieman, C., and Perkins, K. (2008). "Students know what physicists believe, but they don't agree: A study using CLASS survey." Physical Review Special Topics - Physics Education Research, 4(020106).

Hammer, D. (2000). "Student resources for learning introductory physics." Am. J. Phys. 68(7), S52-S59. 
Hatano, G., and Inagaki, K. (1986). "Two courses of expertise", H. Stevenson, H. Azuma, and K. Hakuta, (eds.), Child development and education in Japan. New York: Freeman, pp. 262272.

Hatano, G., and Oura, Y. (2003). "Reconceptualizing school learning using insight from expertise research." Educational Researcher 32(8), 26-29.

Heller, J., and Reif, F. (1984). "Prescribing effective human problem solving processes: Problem description in physics." Cognition and Instruction 1(2), 177-216.

Hestenes, D., and Halloun, I. (1995). "Interpreting the force concept inventory." The Physics Teacher, 33, 502-506.

Hestenes, D., Wells, M., and Swackhamer, G. (1992). "Force concept inventory." The Physics Teacher, 30(3), 141-158.

Hmelo-Silver, C., Duncan, R., and Chinn, C. (2007). "Scaffolding and Achievement in ProblemBased and Inquiry Learning: A Response to Kirschner, Sweller, and Clark (2006)." Educational Psychologist 42(2), 99-107.

Ireson, G. (2000.) "The quantum understanding of pre-university physics students." Phys. Educ. 35(1), 15-21.

Johnston, I. D., Crawford, K., and Fletcher, P. R. (1998). "Student difficulties in learning quantum mechanics." Int. J. Sci. Educ. 20, 427-446.

Jolly, P., Zollman, D., Rebello, S., and Dimitrova, A. (1998). "Visualizing motion in potential wells." Am. J. Phys. 66(1), 57-63.

Kirschner, P., Sweller, J., and Clark, R. (2006). "Why minimal guidance during instruction does not work: an analysis of the failure of constructivist, discovery, problem-based, experiential, and inquiry-based teaching." Educational Psychologist 41(2), 75-86.

Landauer, T. K., and Bjork, R. A. (1978). "Optimum rehearsal patterns and name learning", M. M. Gruneberg, P. E. Morris, and R. N. Sykes, (eds.), Practical aspects of memory. London: Academic Press, pp. 625-632.

Leonard, W., Dufresne, R., and Mestre, J. (1996). "Using qualitative problem-solving strategies to highlight the role of conceptual knowledge in solving problems." Am. J. Phys. 64, 1495-1503.

Lin, S. Y., and Singh, C. (2009). "Assessing Expertise in Quantum Mechanics using Categorization Task", In 2009 Physics Education Research Conference, C. Henderson, M. Sabella, and C. Singh, (eds.), AIP Conf. Proc. 1179, pp. 185-188.

Lin, S. Y., and Singh, C. (2010). "Categorization of quantum mechanics problems by professors and students." Euro. J. Phys. 31, 57-68. 
Luchins, A. S. (1942). "Mechanization in problem solving." Psychological Monographs 54 (Whole No. 248).

Maloney, D. (1994). "Research in problem solving: Physics", D. Gabel, (ed.) Handbook of Research on the Teaching and Learning of Science. New York: MacMillan.

Mason, A., and Singh, C. (2009). "Reflection and Self-monitoring in Quantum Mechanics", In 2009 Physics Education Research Conference 1179, C. Henderson, M. Sabella, and C. Singh, (eds.), AIP Conf. Proc., pp. 197-200.

Mason, A., and Singh, C. (2010). "Do advanced physics students learn from their mistakes without explicit intervention?" Am. J. Phys., 78(7), 760-767.

Mazur, E. (1997). Peer Instruction: A User's Manual, Upper Saddle River, NJ: Prentice Hall.

McDermott, L. C. (1991). "Millikan Lecture 1990: What we teach and what is learned--Closing the gap." Am. J. Phys. 59(4), 15.

McKagan, S. B., Perkins K. K., and Wieman, C. E. (2008a). "A deeper look at student learning of quantum mechanics: the case of tunneling." Physical Review Special Topics - Physics Education Research, 4(020103) and the references therein.

McKagan, S. B., Perkins K. K., Dubson, M., Malley, C., Reid, S., LeMaster, R., and Wieman, C. E. (2008b). "Developing and Research PhET simulations for Teaching Quantum Mechanics." Am. J. Phys., 76, 406-417.

McKagan, S. B., Perkins K. K., and Wieman, C. E. (2008c). "Why we should teach the Bohr model and how to teach it effectively." Physical Review Special Topics - Physics Education Research, 4(010103).

McKagan, S. B., Handley, W., Perkins K. K., and Wieman, C. E. (2009). "A Research-Based Curriculum for Teaching the Photoelectric Effect." Am. J. Phys., 77(1), 87-94 and the references therein.

Melton, A. W. (1970). "The situation with respect to the spacing of repetitions and memory." Journal of Verbal Learning and Verbal Behavior, 9, 596-606.

Mestre, J., Dufresne, R., Gerace, W., Hardiman, P., and Touger, J. (1993). "Promoting skilled problem solving behavior among beginning physics students." Journal of Research in Science Teaching 30, 303-317.

Metcalfe, J., and Shimamura, A. (1994). Metacognition: knowing about knowing, Cambridge, MA: MIT Press.

Miller, G. (1956). "The magical number seven, plus or minus two: Some limits on our capacity for processing information." Psychological Review, 63, 81-97. 
National Research Council. (1996). National Science Education Standards. Washingtion D.C.: National Academy of Sciences

Peterson, L. R., and Peterson, M. J. (1959). "Short-term retention of individual verbal items." Journal of Experimental Psychology 58, 193-198.

Piaget, J. (1964). "Development and learning", R. Ripple and V. Rockcastle, (eds.), Piaget Rediscovered. New York: Cornell University Press, pp. 29.

Raymond, E. (2000). Cognitive Characteristics, Needham Heights, MA: Allyn \& Bacon, A Pearson Education Company.

Redish, E., Saul, J., and Steinberg, R. (1998). "Student Expectations in Introductory Physics." Am. J. Phys., 66, 212-224.

Redish, E., Steinberg, R., and Wittmann, M. (2001). A New Model Course in Applied Quantum Physics, available at http://www.physics.umd.edu/perg/qm/qmcourse/NewModel

Schommer, M. (1990). "Effects of beliefs about the nature of knowledge on comprehension." Journal of Educational Psychology, 82(3), 406-411.

Schwartz, D., Bransford, J., and Sears, D. (2005). "Efficiency and Innovation in Transfer", J. Mestre, (ed.) Transfer of Learning: Research and Perspectives. Greenwish, CT: Information Age Publishing Inc., pp. 1-52.

Scott, M., Stelzer, T., and Gladding, G. (2007) "Explicit Reflection in an Introductory Physics Course." In 2007 Physics Education Research Conference, L. Hsu, C. Henderson, and L. McCullough, (eds.), AIP Conf. Proc. 951, pp. 188-191.

Simon, H. (1974). "How big is a memory chunk." Science, 183(4124), 482-488.

Singh, C. (2005). "Impact of peer interaction on conceptual test performance." Am. J. Phys., 73(5), 446-451.

Singh, C. (2007). "Student difficulties with quantum mechanics formalism" In 2007 Physics Education Research Conference, L. Hsu, C. Henderson, and L. McCullough, (eds.), AIP Conf. Proc. 883, pp. 185-188.

Singh, C., Belloni, M., and Christian, W. (2006). "Improving student's understanding of quantum mechanics." Physics Today, 8(43-49).

Smith, L. (1985). "Making Educational Sense of Piaget's Psychology." Oxford Review of Education, 11(2), 181-191.

Styer, D. (1996). "Common misconceptions regarding quantum mechanics" Am. J. Phys., 64, 3134. 
Touger, J., Dufresne, R., Gerace, W., Hardiman, P., and Mestre, J. (1995). "How novice physics students deal with explanations." International Journal of Science Education, 17(2), 255269.

Van Heuvelen, A. (1991). "Overview Case Study Physics." Am. J. Phys., 59, 898-907.

Wittmann, M., Steinberg, R., and Redish, E. (2002). "Investigating student understanding of quantum mechanics: Spontaneous models of conductivity." Am. J. Phys., 70(3), 218-226.

Yerushalmi, E., and Eylon, B. (2003) "Assessing reflection on practice: a problem solving perspective." In 2003 Physics Education Research Conference, J. Marx, S. Franklin, and K. Cummings, (eds.), AIP Conf. Proc.720, pp. 153-156.

Zollman, D., Rebello, S., and Hogg, K. (2002). "Quantum mechanics for everyone: Hands-on activities integrated with technology." Am. J. Phys., 70(3), 252-259. 


\title{
2.0 IMPROVING STUDENTS’ UNDERSTANDING OF POSSIBLE WAVEFUNCTIONS
}

\begin{abstract}
2.1 ABSTRACT
In this chapter, we will describe the difficulties students have with possible wavefunctions. We will also discuss the development and implementation of a research-based Quantum Interactive Learning Tutorial (QuILT) to reduce these difficulties. The preliminary evaluation shows that the QuILT about possible wavefunctions is effective in improving students' understanding of the concepts related to possible wavefunctions.
\end{abstract}

\subsection{BACKGROUND}

The wavefunction is one of the most fundamental concepts in quantum mechanics. In Newtonian mechanics, once we know the position $x(t=0)$ and velocity $v(t=0)$ of a particle with mass $m$ at a given time $t=0$ and the force $F(x, t)$ acting on it as a function of time, we can at least theoretically figure out the position $x(t)$ and velocity $v(t)$ for all future times and from that information derive other dynamics variables as a function of time, e.g., momentum and kinetic energy. In quantum mechanics on the other hand, a particle is represented as a "probability wave" which is described by the wavefunction $\Psi(x, t)$ at a given time $t$. The absolute square of 
the wavefunction $|\Psi(x, t)|^{2}$ gives the probability density of finding the particle at position $x$, at time $t$. The wavefunction $\Psi(x, t)$ itself is in general complex so its absolute square is

$$
|\Psi(x, t)|^{2}=\Psi^{*}(x, t) \Psi(x, t),
$$

where $\Psi^{*}(x, t)$ is the complex conjugate of the wavefunction $\Psi(x, t)$.

Any possible wavefunction of a quantum system must satisfy some basic properties. First, since $|\Psi(x, t)|^{2}$ represents the probability density of finding the particle at position $x$ and the probability of finding the particle anywhere in space must be unity, the possible wavefunction $\Psi(x, t)$ for any quantum system must be normalizable, i.e., for one spatial dimension,

$$
\int_{-\infty}^{+\infty}|\Psi(x, t)|^{2} d x=1
$$

This implies that the wavefunction must be square integrable and must go to zero at plus and minus infinity. Second, there cannot be two different values of the probability density for finding the particle at a given position $x$. So the wavefunction $\Psi(x, t)$ (both its real and imaginary parts) must be continuous everywhere. Also, the wavefunction must satisfy the boundary conditions of the quantum system. For example, $\Psi(x, t)$ must be zero at the boundary of a one dimensional (1D) infinite square well since the potential is infinite beyond the boundaries of the well. Moreover, the first derivative of the wavefunction $\partial \Psi / \partial x$ must be continuous everywhere except for the positions where the potential energy $V(x)$ is infinite. This is because the kinetic energy operator depends on the second derivative of the wavefunction and is given by

$$
\hat{p}^{2} / 2 m=-\frac{1}{2 m} \frac{\partial^{2}}{\partial x^{2}}
$$


If $\partial \Psi / \partial x$ is discontinuous at a position where the potential energy $V(x)$ is finite, the expectation value of the kinetic energy of the particle would be infinite.

A possible wavefunction of a quantum system can be written as a linear superposition of a complete set of basis vectors. Since eigenfunctions of an operator corresponding to a physical observable, e.g., energy, form a complete set of basis vectors, we can always write a possible wavefunction in terms of a linear superposition of the energy eigenfunctions for that system. The energy eigenfunctions $\psi_{n}(x)(n=1,2,3, \ldots)$ corresponding to the energies $E_{n}$ satisfy the TimeIndependent Schrödinger Equation (TISE)

$$
\hat{H} \psi_{n}(x)=E_{n} \psi_{n}(x) .
$$

Since the time evolution of a quantum system is governed by the Time-Dependent Schrödinger Equation (TDSE), the energy eigenfunction at time $t$ can be represented by the energy eigenfunction at time $t=0$ multiplied by a common phase factor, i.e.,

$$
\psi_{n}(x, t)=\psi_{n}(x, 0) e^{-i E_{n} t / \hbar} .
$$

When a quantum system is in an energy eigenstate, the expectation value of any observable $\hat{Q}$ (without explicit time dependence) is time-independent because the common phase factor cancels out, i.e.,

$$
\langle\hat{Q}(t)\rangle=\int_{-\infty}^{+\infty} \psi_{n}^{*}(x, t) \hat{Q} \psi_{n}(x, t)=\int_{-\infty}^{+\infty} \psi_{n}^{*}(x, 0) e^{+i E_{n} t / \hbar} \hat{Q} \psi_{n}(x, 0) e^{-i E_{n} t / \hbar}=\langle\hat{Q}(t=0)\rangle .
$$

Therefore, the energy eigenfunctions are also called the stationary state wavefunctions.

However, all stationary state wavefunctions are not possible wavefunctions for a quantum system. For example, the stationary state wavefunction of a free particle is a plane wave $e^{i k x}$ where $k$ is the wave vector. This wavefunction is not normalizable so it is not a possible wavefunction for a free particle. However, a normalizable free particle wave packet can be 
constructed by taking a linear superposition of the stationary state wavefunctions $e^{i k x}$ with different wave vectors $k$.

\subsection{INVESTIGATION OF STUDENTS' DIFFICULTIES}

In these investigations, our goal was to examine students' difficulties with possible wavefunctions after traditional instruction so that we can devise strategies to improve students' understanding. The investigation of students' difficulties with possible wavefunctions was carried out by administering written surveys to more than a hundred advanced undergraduate and graduate students enrolled in quantum mechanics courses and by conducting individual interviews with a subset of students. Both open-ended questions and multiple-choice questions were administered to probe students' difficulties. The individual interviews were conducted using a think-aloud protocol (Chi 1994). During the think-aloud interviews, students were asked to verbalize their reasoning process while they answered the questions about possible wavefunctions. They were not interrupted unless they remained silent for a while. At the end of the interview, we asked the students to clarify issues they had not made clear in their earlier explanations.

\subsubsection{Difficulties related to the normalization of possible wavefunctions}

One survey question asked students to draw a qualitative sketch of the ground state wavefunction of a particle in a $1 \mathrm{D}$ finite square well of width $a$ and depth $-V_{0}\left(V_{0}>0\right)$ between $0 \leq x \leq a$. We note that though students were provided separate spaces for drawing the wavefunction, they 
still confused the vertical axis in the potential energy diagram with the vertical axis of the wavefunction. Instead of simply showing the location of $x=0$ and $x=a$ in their sketches, many students redrew the potential energy diagrams and situated the wavefunction in the well without specifying what the vertical axes of their plots were. Such confusion between the vertical axis of the potential energy diagram and the vertical axis of wavefunction indicates that students may have difficulties with interpreting the dimension (unit) of the wavefunction. For a possible wavefunction $\Psi(x, t)$ corresponding to a quantum system in one spatial dimension, the normalization condition (Eq. 2.2) must be satisfied, which implies

$$
\text { unit of } \Psi(x, t)=1 / \sqrt{\text { unit of } x} \text {, }
$$

in which the position $x$ has the unit of length.

Another question in the investigation asked students to draw the wavefunction of the particle in a 1D finite square well when the energy of the particle is higher than zero (the potential energy $V(x)$ of the finite square well is $V(x)=-V_{0}$ between $0 \leq x \leq a$ and $V(x)=0$ elsewhere). In response to this question, some students incorrectly claimed that the slope of the wavefunction is zero outside the well since the potential energy there is zero (e.g., Figure 2.1). The student who sketched Figure 2.1 also incorrectly believed that the constant value of the wavefunction in the region III is lower compared to region I since it is affected by the potential energy in region II and “dies”.
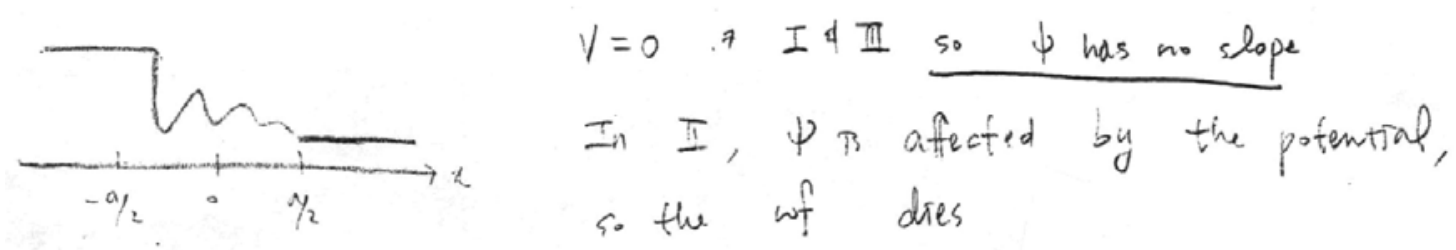

Figure 2.1 According to this student, the slope of the wavefunction is zero in the regions where potential energy is zero. 
Some students also had difficulty with normalization of the wavefunction and why normalization is important. For example, some students were confused about the normalization issues of a free particle stationary state wavefunction. Since the stationary state wavefunction of a free particle cannot be normalized, it is not a possible wavefunction for a free particle. Some students were confused about whether a free particle can be in a stationary state. For a free particle, we can form a wave packet which is a superposition of the energy eigenstates. If these wavepackets are formed by taking a linear superposition of stationary states in a very narrow range of the energy spectrum, it can be made normalizable but still considered to be almost a stationary state wavefunction. Thus, although a stationary state wavefunction of a free particle is not a possible wavefunction, the free particle can still have its energy in a very narrow range.

\subsubsection{Difficulties related to the boundary conditions in different potential energy wells}

The one dimensional (1D) infinite square well and 1D finite square well are common models used to illustrate the basic machinery of quantum mechanics. However, students have difficulties in differentiating between the possible wavefunctions for the finite and infinite square wells. For example, when asked to draw the ground state wavefunction for a finite square well, some interviewed students claimed that the shape of the various bound state wavefunctions for the finite square well cannot be sinusoidal inside the well because the sinusoidal stationary state wavefunctions are only possible for an infinite square well. One of the students incorrectly sketched a Gaussian function and claimed that the ground state wavefunction should be Gaussian to ensure that the wavefunction has no cusp and decays to zero outside the well. However, solving the TISE for the finite square well, one finds that the stationary state wavefunctions inside the finite square well are sinusoidal functions. We find that while students noticed some 
differences between the finite and infinite square well stationary state wavefunctions correctly, they overlooked some features that these two models have in common.

When analyzing the possible wavefunctions for a finite square well, some students overgeneralize what they have learned about the stationary state wavefunctions for that potential energy well. For example, the stationary state wavefunctions for a finite square well have nonzero exponential tails (bound states) or oscillatory behavior (scattering states) in the region outside the well. Students often incorrectly over-generalize the behavior of the stationary state wavefunctions and conclude that any possible wavefunction for a finite square well must have a non-zero part outside the well. For example, in a multiple choice question, we asked 85 students whether a normalized wavefunction as shown in Figure 2.2 is a possible wavefunction for a finite square well between $0<x<a$. The wavefunction $\psi(x)$ shown is zero in the regions $x<b_{1}$ and $x>b_{2}$. Only $40 \%$ of the students correctly answered that such a wavefunction is a possible wavefunction for the finite square well. About $51 \%$ of the students chose the wrong statement that "it is not a possible wavefunction because the probability of finding the particle outside the finite square well is zero but quantum mechanically it must be nonzero". Among those students who incorrectly believed that the wavefunction must be nonzero outside the well, $60 \%$ also claimed that the wavefunction in Figure 2.2. does not satisfy the boundary condition of the finite square well.

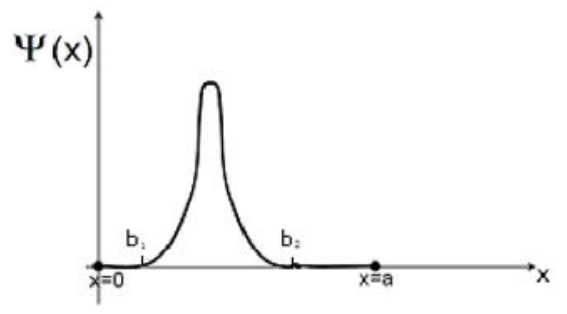

Figure 2.2 A wavefunction localized inside a finite square well. Students incorrectly believed that any possible wavefunction in a finite square well must have non-zero value outside the well. 


\subsubsection{Difficulties related to the continuity of possible wavefunction}

Students often neglect the requirement of the continuity of the wavefunction when they sketch the wavefunction for a particle interacting with a piecewise continuous potential energy such as the finite square well. For example, in Figures 2.3(a) and (b), both students realized that the ground state wavefunction is sinusoidal inside the finite square well and decaying outside the well. However, they drew the wavefunction inside and outside the well separately without ensuring that the wavefunction is continuous at the boundaries between different regions. In Figure 2.3(c), a student sketched a scattering state wavefunction of a particle incident from the left side of the well. The wavefunction should be oscillatory in all the three regions (including inside the well) instead of exponentially decaying as drawn by the student. Also, the student who drew Figure 2.3(c) incorrectly sketched a discontinuous wavefunction at the left boundary of the well.
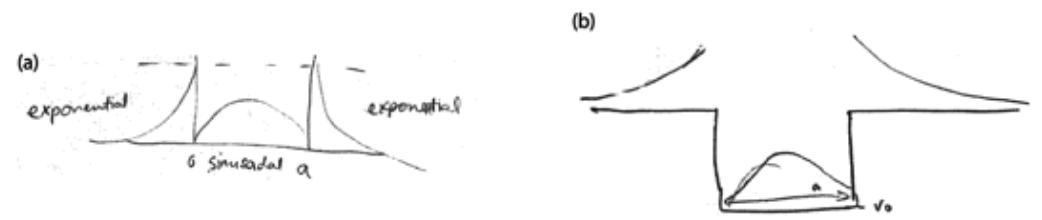

(c)

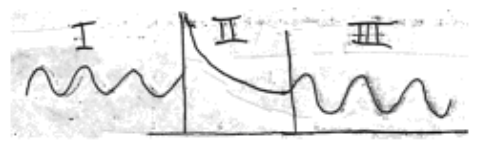

Figure 2.3 Samples of incorrect sketches of wavefunctions which are discontinuous at the boundaries of the finite square well. 


\subsubsection{Difficulties related to the continuity of the first derivative of a possible wavefunction}

Compared to the continuity of the wavefunction, the continuity of the first derivative of the wavefunction is more difficult for the students to internalize. Many students do not correctly interpret the meaning of the first derivative of the wavefunction $\partial \Psi / \partial x$. Since the derivative of $\partial \Psi / \partial x$ (or the second derivative of $\Psi$ ) is related to the kinetic energy of the system, the wavefunction must be smooth everywhere except where the potential energy is infinite. For example, in a 1D infinite square well between $0 \leq x \leq a$, the first derivative of the stationary state wavefunctions are continuous between $0<x<a$ but discontinuous at the boundaries $x=0$ and $x=a$. Thus, a possible wavefunction for an infinite square well should not have any cusp inside the well. However, when we asked the students whether the function as shown in Figure 2.4 was a possible wavefunction for an infinite square well, four out of seven students claimed that it is a possible wavefunction even though the question explicitly mentioned the discontinuity of $\partial \Psi / \partial x$ at the position $x=b$ inside the well. Some students made similar mistakes when they were asked to draw the ground state wavefunction for a finite square well as shown in Figure 2.5.

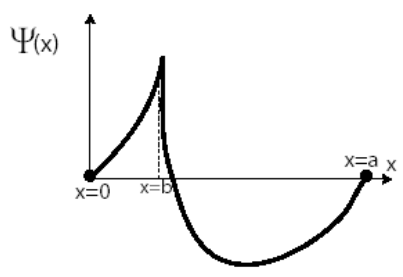

Figure 2.4 A wavefunction with a cusp inside the well is not allowed for an infinite square well.

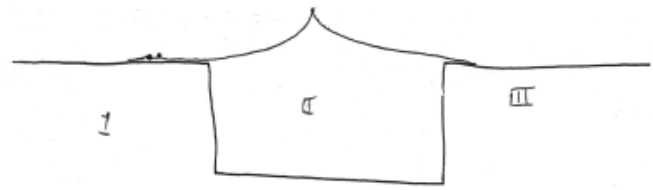

Figure 2.5 Wavefunction with a cusp inside the well drawn by a student. The wavefunction is not allowed for a finite square well. 
Some students who did not realize that the first derivative of the wavefunction must be continuous where the potential energy is finite over-generalized their experience about the discontinuity of $\partial \Psi / \partial x$ at the boundaries of an infinite square well and incorrectly believed that $\partial \Psi / \partial x$ can be discontinuous at the boundaries of a finite square well as well. For example, when students were given a question asking them to draw a wavefunction that is possible for both infinite and finite square wells, a student sketched a stationary state wavefunction for the infinite square well and claimed that "it (the stationary state wavefunction for the infinite square well) also works for finite square wells”.

\subsubsection{Difficulties with qualitative sketch of the possible wavefunction without using quantitative solutions}

Some students have difficulties with qualitatively sketching the possible wavefunction if they do not know the quantitative solution of the Time-Independent Schrödinger Equation (TISE) for the system. During the interview, one student claimed that it is impossible to draw the stationary state wavefunctions for a finite square well because one must find the solution of a transcendental equation which can only be numerically solved. When the student was encouraged to make a qualitative sketch, he drew two coordinate axes and then drew some parallel curves and a straight line from the origin intercepting the curves (Figure 2.6). He claimed that all he can say without solving the equation numerically is that the intercepts will give the wavefunction. While one must solve a transcendental equation to find the finite number of bound states for a finite square well, the student was asked to draw a qualitative sketch of the wave function, something that is taught even in a modem physics course. In particular, students are taught that the bound state wave functions for a finite square well look sinusoidal inside the 
well with an exponential tail outside in the classically forbidden region. It appeared that the student had memorized a procedure but had not developed a qualitative "feel" for what the bound and scattering state wave functions should look like for a finite square well.

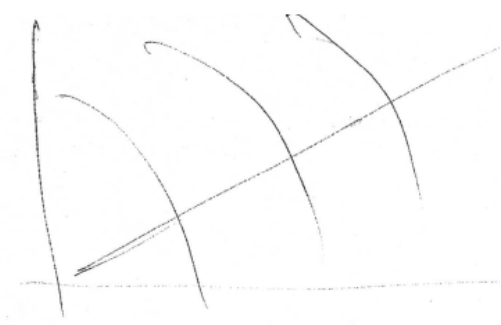

Figure 2.6 A student's sketch to find a graphical solution of the transcendental equation which he believed was necessary to obtain a qualitative sketch of the ground state wavefunction for the finite square well potential energy.

\subsection{RESEARCH BASED LEARNING TOOLS}

Based on the investigation of students' difficulties, we developed a QuILT to improve students' understanding of possible wavefunctions. The goal of the possible wavefunction QuILT is to help students learn about possible wavefunctions and bridge the gap between conceptual and quantitative aspects. The development of the QuILT went through a cyclical iterative process which includes the following stages: (1) Development of the preliminary version based upon theoretical analysis of the underlying knowledge structure and research on students' difficulties with possible wavefunctions, (2) implementation and evaluation of the QuILT by administering it individually to students, measuring its impact on student learning and assessing what difficulties remained, (3) refinement and modification based upon the feedback from the implementation and evaluation. 
As noted in the introduction section, the QuILT uses a guided approach to learning about the possible wavefunctions for a given potential energy and it takes advantage of students' prior knowledge and resources about wavefunction found during investigation of students' difficulties. The QuILT also uses computer-based visualization tools to help students develop physical intuition about the possible wavefunction for different potential energies. The simulations adapted in the QuILT related to the infinite square well and the free particle wave packets are developed by the Open Source Physics project (Christian \& Belloni 2008), which is flexible and can be easily tailored to the desired situations in our QuILT. We also adapted a PhET Interactive Simulation developed at the University of Colorado in our QuILT (McKagan et al, 2009).

\subsubsection{Possible wavefunction for a $1 \mathrm{D}$ infinite square well}

The first part of the QuILT discusses the possible wavefunction in the simplest model involving an infinite dimensional Hilbert space, i.e., the 1D infinite square well. At the beginning of the QuILT, students get an opportunity to review the properties of the stationary state wavefunctions $\psi_{n}(x)$ and judge whether the superposition of the stationary state wavefunctions (presented both in the mathematical and pictorial representations) are possible wavefunctions for the infinite square well at a given time. During the investigation of student difficulties, we found that many students could recognize that the superposition of the stationary state wavefunctions in the mathematical representation is a possible wavefunction. But they incorrectly believed that the same wavefunction in the graphical representation is not possible because the graph is not symmetric or anti-symmetric about the center of the well. Therefore, one question in the QuILT 
is designed in the mathematical representation, e.g., $\frac{1}{\sqrt{2}}\left[\psi_{1}(x)+\psi_{2}(x)\right]$, and another one in the graphical representation as shown in Figure 2.7.

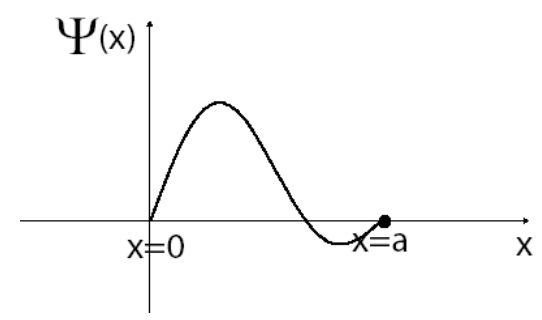

Figure 2.7 A superposition of stationary state wavefunctions for a 1D infinite square well.

To ensure that students understand why the asymmetric wavefunction is a possible wavefunction even though the potential energy is symmetric about the center of the well, we help students connect the mathematical and graphical representations. We also ask them to consider a dialogue in which students have to make sense of a conversation between two people as follows:

Sally: I don't understand the answer to question above (Figure 2.7). The wavefunction is neither symmetric nor anti-symmetric about the center of the well. Why is it a possible wavefunction for a symmetric potential energy?

Harry: When the potential energy is symmetric, an energy eigenfunction must be symmetric or anti-symmetric. But a superposition of energy eigenstates is not necessarily symmetric or anti-symmetric. For example, the sum of an even function and an odd function is neither even nor odd.

Sally: But I think a possible wavefunction must be an eigenstate of a particular operator, e.g., the Hamiltonian or position operator.

Harry: That's not true. The possible wavefunction need not be an eigenstate of a particular operator. It can be a superposition of the eigenstates.

Do you agree with Sally or Harry? Explain. 
The QuILT also asks students to use the simulations to construct various superpositions of the stationary state wavefunctions for the infinite square well so that they can watch how the symmetric/anti-symmetric stationary state wavefunctions combine into other possible wavefunctions that are neither symmetric or anti-symmetric. A snapshot of the simulation is shown in Figure 2.8. The first window shows the absolute value of the wavefunction. Students can change the width of the infinite square well and start/stop the time evolution to observe how the wavefunction changes with time. The option "phase as color" should be selected in our QuILT. Unselecting this option shows the real and imaginary parts of the wavefunction separately. The second window shows the coefficients in the superposition. The coefficients of different energy eigenstates (marked by “quantum \#”) can be inputted to build a wavefunction which is a linear superposition of stationary states. "Re" is the real part and "Im" is the imaginary part of the coefficients. Students can use the button "Normalize" at the bottom of the second window to normalize the coefficients.
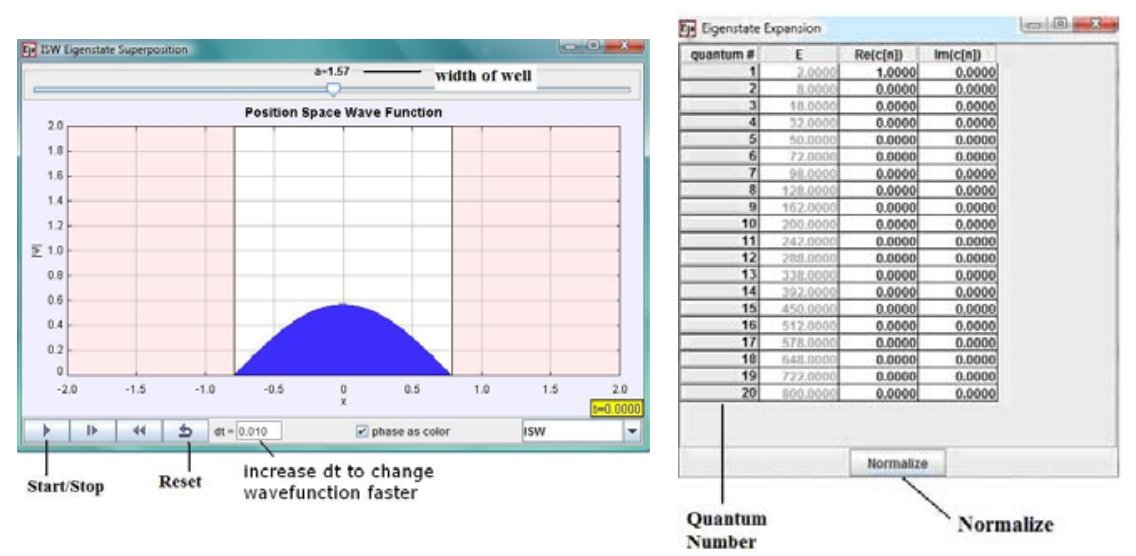

Figure 2.8 OSP simulation of the possible wavefunction in a 1D infinite square well.

Students are asked to first predict the qualitative shape of various superpositions of the stationary state wavefunctions and then use the simulation to examine their prediction. Students also must reconcile the differences between their prediction and what they observe in the simulation. The following is a sample excerpt: 
Predict and sketch the shape of the wavefunction $\Psi(x)=\frac{1}{\sqrt{2}}\left[\psi_{1}(x)-\psi_{2}(x)\right]$.

Input 1 as the coefficient for the ground state and -1 for the first excited state in the simulation coefficient box. Click "normalize" and observe the absolute value of the superposition wavefunction. Is the shape the same as your prediction? Explain. (Note that the simulation shows the absolute value of the wavefunction.)

After the students use the simulation to build a superposition of the stationary state wavefunctions, they are given several multiple choice questions to review the properties of a possible wavefunction in an infinite square well. For example, students often incorrectly believe that any possible wavefunction must satisfy the TISE. In the QuILT, we ask students to consider whether the wavefunction $\Psi(x, 0)=\frac{1}{\sqrt{2}}\left[\psi_{1}(x)+\psi_{2}(x)\right]$ satisfies the TISE. After their prediction, students must check their prediction by explicitly plugging the wavefunction in the TISE. They are also asked to find the wavefunction at time $t$ and then plug it into the Time-Dependent Schrödinger Equation (TDSE) to check whether $\Psi(x, t)$ satisfies the TDSE. They learn that a possible wavefunction $\Psi(x, t)$ always satisfies the TDSE but not necessarily the TISE (unless it is a stationary state or a superposition of stationary states with the same energy). Students are also asked to predict how different possible wavefunctions for an infinite square well evolve with time and they use the simulations in the QuILT to check their prediction. This activity is particularly useful in helping students understand the difference between the time evolution of the probability density for a stationary state wavefunction and a superposition of the stationary state wavefunctions. 


\subsubsection{Possible wavefunction for a 1D finite square well}

The stationary state wavefunctions for an infinite square well are in the form of sinusoidal waves inside the well and zero outside the well. It is a simple model to help students understand that the possible wavefunction for a quantum system can be written as a linear superposition of the stationary state wavefunctions. However, the possible wavefunctions for an infinite square well are always zero in the classically forbidden region. Moreover, due to the potential energy being infinite outside the well, first derivative of the wavefunction is not continuous at the boundaries. These artificially constrained properties of the infinite square well are not true for more realistic potential energies. Therefore, students must learn the properties of the possible wavefunctions for more realistic potential energies, e.g., the 1D finite square well.

While discussing the 1D finite square well, students are asked to consider whether a wavefunction with a discontinuous first derivative is a possible wavefunction (as shown in Figure 2.9). Students learn that it is a possible wavefunction for an infinite square well but not for a finite square well since its first derivative is discontinuous at $x=0$ and $x=a$.

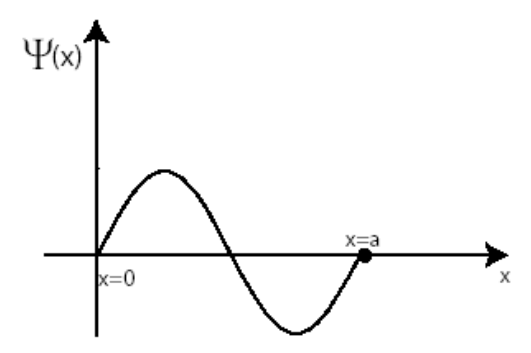

Figure 2.9 The first derivative of the wavefunction is discontinuous at the boundaries so this wavefunction is not a possible wavefunction for a finite square well.

Students use the simulation to observe the shape of the stationary state wavefunctions for a finite square well. They can also build a wavefunction which is a linear superposition of the stationary state wavefunctions in the simulation and compare the difference between the possible 
wavefunctions for the finite square well and the infinite square well. We adapted the quantum bound state program (see snapshot in Figure 2.10) developed by the PhET team at the University of Colorado in the QuILT. Students can change the depth and width of the 1D finite square well and select a particular energy level to observe the absolute square of an energy eigenfunction. Students can also build various linear superpositions of stationary states by clicking the button "Superposition State" and inputting the coefficients as desired. Students can also observe the time evolution of the absolute square of the wavefunction (probability density) by clicking the button “Play/Pause”. In the QuILT of the possible wavefunction, students are asked to predict the outcomes, e.g., the qualitative shape of the superposition state $\frac{1}{\sqrt{2}}\left[\psi_{1}(x)+\psi_{2}(x)\right]$ in a finite square well and then check their prediction using the simulation.

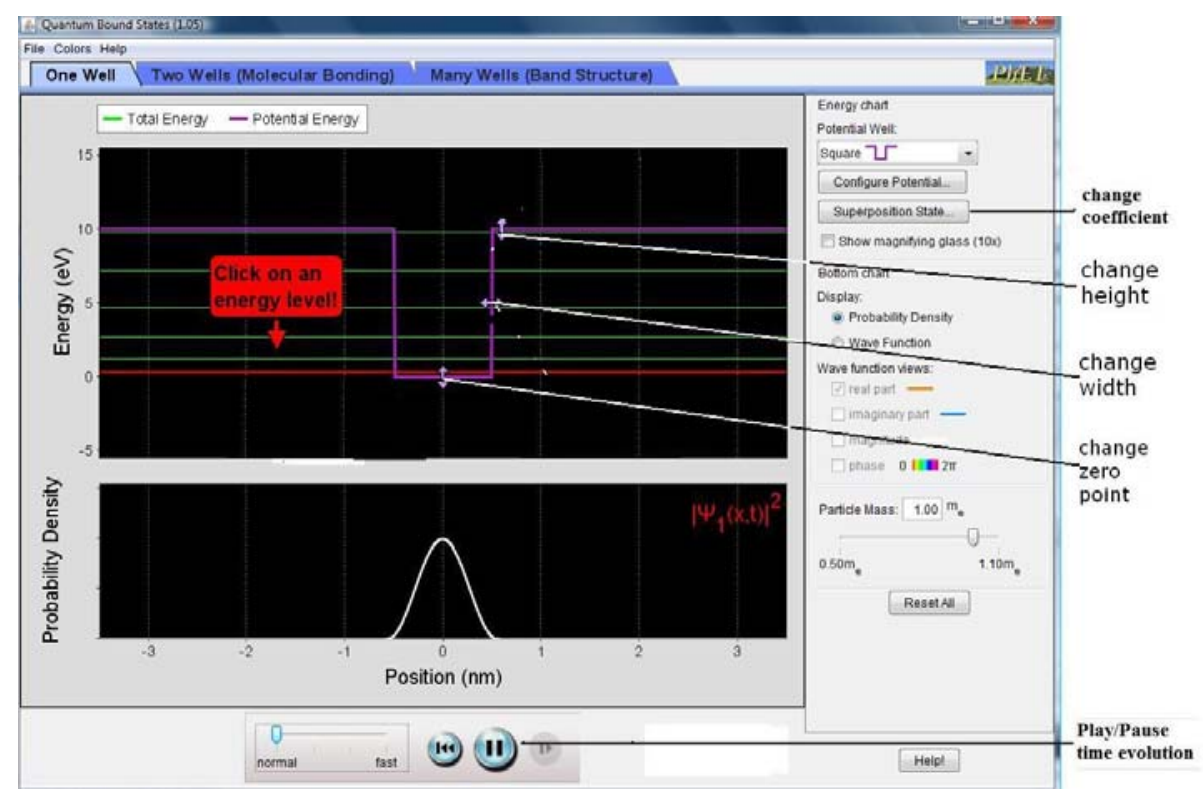

Figure 2.10 A snapshot of the simulation of the finite square well.

Figure 2.11 shows an example in which students are given two similar non-stationary wavefunctions (but one stretched to the left with respect to the other) to learn that a possible wavefunction in a finite square well can be nonzero beyond the well and need not be symmetric 
about the center of the well. Earlier in the QuILT, in the context of the infinite square well, students are asked to consider the same two wavefunctions and determine if they are possible wavefunctions. As discussed in chapter 2.3.2, some students incorrectly believe that the wavefunction must be non-zero outside a finite square well because of the fact that the stationary state wavefunctions for this system always have a non-zero part outside of the well. We therefore also asked students whether a peaked wavefunction as shown in Figure 2.2 is a possible wavefunction for a finite square well. For all the three wavefunctions (Figure 2.2, Figure 2.11(a), Figure 2.11(b)) for a finite square well, students learn that they are possible wavefunctions because they are "continuous, smooth and normalizable". They learn that just because the stationary state wavefunctions have a non-zero part outside of the well does not imply that we cannot take their linear superpositions to form possible wavefunctions that are zero outside the well.
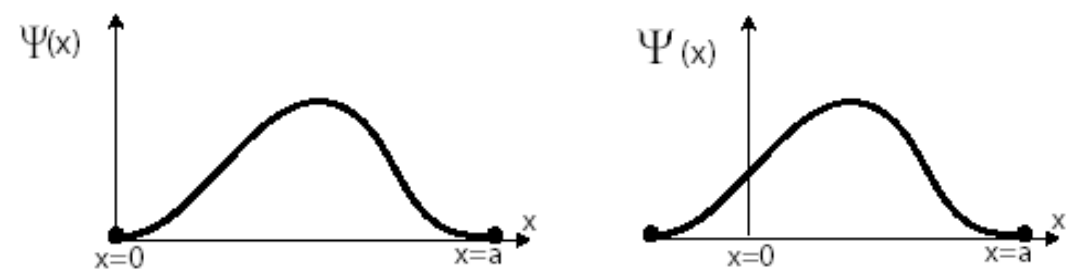

Figure 2.11 (a) The wavefunction is zero in the classically forbidden region of a finite square well.

(b) The wavefunction is non-zero in the classically forbidden region of a finite square well.

Students again use the simulations to check the time evolution of the stationary states and non-stationary states for a finite square well. Students are also asked to sketch, e.g., the ground state wavefunctions for the finite and infinite square wells and compare and explain the similarities and differences between these wavefunctions. 


\subsubsection{Possible wavefunction for a free particle}

In some quantum systems, e.g., a free particle, the stationary state wavefunctions are not normalizable. Although the possible wavefunctions in such quantum systems are a linear superposition of the stationary states, the stationary states themselves are not possible wavefunctions. In the QuILT on possible wavefunctions, we use the free particle system to help students understand these issues.

In the QuILT, students are asked to choose the correct graphical representation of the stationary state wavefunction of a free particle and their attention is drawn to the fact that the stationary state wavefunctions $A e^{i k x}$ for a free particle are not normalizable. We provided a dialogue question as shown below to help the students understand why an energy or momentum eigenstate wavefunction for a free particle is not a possible wavefunction but we can still have a free particle with definite energy or momentum.

Sally: How can the energy eigenfunction not be a possible wave function for the free particle?

Harry: Because the absolute square of the wavefunction must be normalizable. Otherwise the total probability of finding the particle would be infinite.

Sally: I disagree. If the energy eigenfunction is not a possible wave function, that means we cannot have a free particle with definite value of energy or momentum. But classically we can always have a free particle moving with a constant momentum.

Harry: Well, the free particles in reality exist as wave packets. The magnitude of the

momentum of a free particle is $p=\hbar k$ and the energy is $E=\frac{\hbar^{2} k^{2}}{2 m}$, where $k=\frac{2 \pi}{\lambda}$ is the magnitude of the wave vector. A wave packet could consist of plane waves $e^{i k x}$ with different 
wave vectors $k$ in a very narrow range. Thus, we can consider the wave packet as "effectively" having a definite energy and momentum, if the distribution of energy/momentum is highly localized about a given wave vector $k$.

Do you agree with Sally or Harry? Explain.

We incorporated another simulation from Open Source Physics to help students learn that a free particle wave packet can be constructed using a linear superposition of the stationary state wavefunctions. Students can observe that the wave packet spreads out as time evolves. Students learn that the spreading of the wave packet is due to different stationary state wavefunctions that form the wave packet having different phase velocities. Students also learn that in condensed matter physics, the free particle model is often used for electrons in metals with periodic boundary conditions imposed on the system.

\subsubsection{QuILT Homework for Possible Wavefunctions}

The QuILT homework helps students review the concepts they have learned in the QuILT. In the homework, students must explain in their own words why the wavefunction must be continuous. Students are given different wavefunctions and asked to judge whether they are possible for the finite or infinite square well and explain their reasoning. They are also asked to differentiate between any possible wavefunction and the stationary state wavefunctions. In the QuILT homework, students are also asked to explain why they agree or disagree with statements about the possible wavefunctions such as the following:

(1) Consider the following statement: For an infinite square well, all possible wavefunctions are energy eigenfunctions because the time independent Schrödinger equation 
(TISE) is an eigenvalue equation for energy. Explain why you agree or disagree with this statement.

(2) Consider the following statements: The energy eigenfunction $A e^{i k x}$ ( $A$ is a constant and $k$ is the wave vector) for a free particle is not a possible wavefunction. Therefore, we cannot represent a possible wavefunction for a free particle as a superposition of functions of the form A $e^{i k x}$. Explain why you agree or disagree with this statement.

\section{$2.5 \quad$ PRE-TEST AND POST-TEST DATA}

We conducted preliminary evaluations of the QuILT about possible wavefunction in two junior-senior level quantum mechanics classes, first with 13 students and second with 18 students. The two classes were taught by the same instructor. In both classes, students first received traditional instruction about the possible wavefunctions for different quantum systems, e.g., 1D infinite square well, 1D finite square well, free particle, etc. After traditional instruction, students took the pre-test and then worked on the QuILT. The post-test was administered in the following class period after students had finished the QuILT. We designed two versions of a test (versions A and B) to assess students' understanding of possible wavefunctions. Both versions A and B have 5 questions each. Students were randomly given either version A or version B of the test as the pre-test after the traditional instruction. Then, each student was administered the version of the test he/she had not taken as the post-test after working on the QuILT. In particular, 15 students in the two classes were administered version A as pre-test (and version B as the posttest) whereas the other 16 students were given version $\mathrm{B}$ as the pre-test (and version $\mathrm{A}$ as the post-test). The average pre-test score for all 31 students was $63 \%$ and the average post-test score 
was $89 \%$. The average pre-test and post-test score on each question combining the two classes is listed in Table 2.1.

Question A-2 asks students to consider whether a wavefunction with a cusp (as shown in Figure 2.4) is a possible wavefunction for an infinite square well. The improved performance suggests that the students are more likely to understand that a possible wavefunction must be smooth. Question B-3 as shown below tested whether students could recognize the discontinuous first derivative of the wavefunction at the boundary which is not possible for a finite square well. The results of question B-3 indicate that after the QuILT, students had a better understanding of these issues.

Question B-3: Select all of the following wave functions which are possible for an electron in a one dimensional finite square well of width $a$ between $x=0$ and $x=a$. A is $a$ suitable normalization constant. You must provide a clear reasoning for each case.

(a) $\Psi(x)=A \sin (3 \pi x / a)$ for $0 \leq x \leq a, \Psi(x)=0$, otherwise.

(b) $\Psi(x)=A(\sqrt{2 / 5} \sin (\pi x / a)+\sqrt{3 / 5} \sin (2 \pi x / a))$ for $0 \leq x \leq a, \Psi(x)=0$, otherwise.

(c) $\Psi(x)=A e^{-((x-a / 2) / a)^{2}}$.

Questions A-4 and B-4 both asked students to consider whether the stationary state wavefunction for a free particle is a possible wavefunction. We gave the mathematical form of the stationary state wavefunction $A e^{i k x}$ for the free particle in question A-4 but not in B-4. The improved performance on both questions in the post-test suggests that students have a better understanding of the normalization issues of the free particle stationary state wavefunctions. In the post-test, some students explicitly mentioned that a possible wavefunction for a free particle can be constructed by forming a wave packet using a linear superposition of the stationary state wavefunctions. 
Question A-5 asked students to sketch a single wavefunction that is possible for both infinite and finite square wells of width $a$. Both wells are between $x=0$ and $x=a$. If students believed that such wavefunctions do not exist then they were asked to explain their reasoning. Partial scores were given to students if they only drew a correct graph but did not provide an explanation. In the pre-test, more than $80 \%$ of the students either sketched incorrect graphs which are not possible for the finite square well or incorrectly claimed that such a wavefunction does not exist. After the QuILT, students realized that a possible wavefunction for both infinite and finite square wells must be zero outside the well and smooth everywhere including at the boundaries of the well. Question B-5 asked the students to sketch a single wavefunction that is possible for the infinite square well but not for a finite square well. Students showed improved understanding of the properties of possible wavefunction in different potential energy wells after the QuILT.

Table 2.1 The pre-test and post-test scores on each question. A and $B$ represent the test version. Fifteen students were administered test $A$ and sixteen students were administered test $B$ in the pre-test and switched the test versions in the post-test.

\begin{tabular}{|c|c|c|c|c|c|c|c|c|c|c|}
\hline Question & A-1 & A-2 & A-3 & A-4 & A-5 & B-1 & B-2 & B-3 & B-4 & B-5 \\
\hline Pre-test Score in \% & $97 \%$ & $60 \%$ & $93 \%$ & $67 \%$ & $17 \%$ & $81 \%$ & $41 \%$ & $44 \%$ & $66 \%$ & $50 \%$ \\
\hline Post-test Score in \% & $94 \%$ & $88 \%$ & $97 \%$ & $97 \%$ & $84 \%$ & $100 \%$ & $93 \%$ & $86 \%$ & $79 \%$ & $75 \%$ \\
\hline
\end{tabular}




\subsection{SUMMARY}

We have investigated students' difficulties related to possible wavefunctions for different quantum systems and used the findings as a guide to develop the QuILT related to possible wavefunctions. Preliminary evaluation suggests that the QuILT about possible wavefunction is effective in improving students' understanding of these concepts.

\subsection{CHAPTER REFERENCE}

Chi, M. (1994). “Thinking Aloud”, M. Van Someren, Y. Barnard, and J. Sandberg (eds.), The Think Aloud Method: A Practical Guide to Modeling Cognitive Processes. Edited by London: Academic.

Christian, W. and Belloni, M. (2008). Simulation program is available at the OSP ComPADRE collection $<$ http://www.compadre.org/osp/items/detail.cfm?ID=6798 $>$

McKagan, S., Malley C., Adams, W., Perkins, K. and Wieman, C. (2009). Simulation program is available at $<\underline{\text { http: } / / \text { phet.colorado.edu }>}$ 


\title{
3.0 IMPROVING STUDENTS' UNDERSTANDING OF BOUND \& SCATTERING STATE WAVEFUNCTIONS
}

\begin{abstract}
3.1 ABSTRACT
In this chapter, we describe the difficulties students have with the bound and scattering state wavefunctions. We also discuss the development and implementation of a research-based Quantum Interactive Learning Tutorial (QuILT) to reduce these difficulties. The preliminary evaluation shows that the QuILT about the bound and scattering state wavefunctions is effective in improving students' understanding of the concepts related to bound and scattering states.
\end{abstract}

\subsection{BACKGROUND}

Energy eigenfunctions $\psi_{n}(x)(n=1,2,3, \ldots)$ corresponding to the energies $E_{n}$ satisfy the TimeIndependent Schrödinger Equation (TISE) for a quantum system with Hamiltonian $\hat{H}$, i.e.,

$$
\hat{H} \psi_{n}(x)=E_{n} \psi_{n}(x) .
$$

The energy eigenfunction at time $t$ can be obtained by multiplying the energy eigenfunction at time $t=0$ by the phase factor $e^{-i E_{n} t / \hbar}$, i.e.,

$$
\psi_{n}(x, t)=\psi_{n}(x, 0) e^{-i E_{n} t / \hbar} .
$$


Since the energy eigenfunctions of a quantum system form a complete set of basis vectors for the Hilbert space, any possible wavefunction of the system can be written as a linear superposition of the energy eigenfunctions for that system. Based on the comparison between the energy of a particle for a given quantum system and the potential energy at plus/minus infinity (in the position space), the energy eigenstates can be categorized into bound states and scattering states. If the energy of the particle is less than the potential energy at both plus and minus infinity, the energy eigenstate is a bound state. Otherwise, the energy eigenstate is a scattering state. When approaching infinity ( $x \rightarrow \pm \infty$ ), a bound state wavefunction for a quantum system decays to zero so the bound state wavefunctions are normalizable. But a scattering state wavefunction is oscillatory at either plus or minus infinity or both so it is not normalizable. However, although a possible wavefunction can be constructed by taking a linear superposition of the scattering states, a scattering state itself is not a possible wavefunction for the quantum system.

\subsection{INVESTIGATION OF STUDENT'S DIFFICULTIES}

In these investigations, our goal was to examine students' difficulties with the bound and scattering state wavefunctions after traditional instruction so that we can devise strategies to improve students' understanding. The investigation of students' difficulties with the bound and scattering state wavefunctions was carried out by administering written surveys to more than two hundred advanced undergraduate and graduate students enrolled in quantum mechanics courses and by conducting individual interviews with a subset of students. We have used both openended questions and multiple-choice questions to probe students' difficulties. The individual interviews were conducted using a think-aloud protocol (Chi 1994). When the students answered 
the questions in the think-aloud interviews, they were asked to verbalize their reasoning process and not interrupted unless they remained silent for a while. At the end of the interview, students were asked to make further explanations on the issues which they had not clarified earlier.

\subsubsection{Difficulties related to the classical bound state and the quantum bound and scattering states}

In a classical system, when the energy of an object is less than the potential energy in the local regions, the object is in a classical bound state. For example, as shown in Figure 3.1, if a toy car with energy $E$ is initially located between $x=a$ and $x=b$, it is bounded in that region and cannot move to other regions such as $x<a$ or $x>b$. The positions where the potential energy $V$ equals the total energy $E$ of the classical object are called the classical turning points, e.g., $x=a$ and $x=b$ in Figure 3.1 (at these points the kinetic energy of a classical particle is zero and the particle turns around). The regions beyond the classical turning points are called the classically forbidden regions such as the regions (I), (II) and (IV) in Figure 3.1.

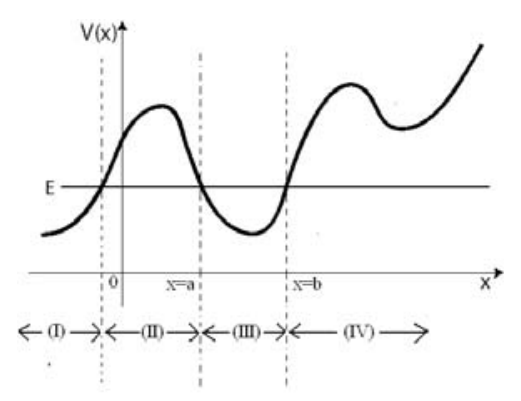

Figure 3.1 An example of classical bound state but quantum mechanical scattering state.

However, for a quantum particle in an energy eigenstate, its wavefunction is non-zero in the classically forbidden regions except where the potential energy is infinity. Therefore, a quantum particle in an energy eigenstate has a finite probability of being found in the classically 
forbidden regions. Many students have difficulties in differentiating between the classical bound and scattering states and the quantum mechanical bound and scattering states. Take the system shown in Figure 3.1 as an example. Some students in our study incorrectly claimed that a quantum particle must stay in region (III) for all times, while some other students mistakenly believed that a classical particle which was initially in region (III) can also be found in region (I).

Many students incorrectly believed that any potential energy that allows a classical bound state must also allow a quantum mechanical bound state. We have given 109 students in seven universities a multiple-choice question asking them whether a quantum mechanical bound state is allowed for the potential energy as shown in Figure 3.2. Only $22 \%$ of the students correctly recognized that no bound state could exist in such a potential energy well since the energy of the quantum particle must be greater than the potential energy at plus/minus infinity.

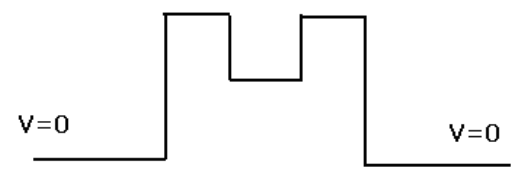

Figure 3.2 A potential energy well that does not allow quantum mechanical bound states.

\subsubsection{Difficulties related to the bound and scattering states being part of the same wavefunction}

Some students have difficulty realizing that a bound state or a scattering state is only determined by comparing the total energy $E$ of a quantum particle in the given state and the potential energy $V$ at plus/minus infinity. Instead, some students claimed that a given quantum particle is in a bound state between the classical turning points and in a scattering state elsewhere, which indicates that these students incorrectly believe that the bound and scattering state wavefunctions are different parts of the same wavefunction. For example, when we asked the students to draw a 
qualitative sketch of a ground state wavefunction and a scattering state wavefunction for a onedimensional (1D) finite square well of width $a$ and depth $-V_{0}$ between $0 \leq x \leq a$, a student sketched a wavefunction as shown in Figure 3.3. He believed that the part of the wavefunction inside the well was a bound state and the parts of the wavefunction outside the well were scattering states corresponding to a "free particle".

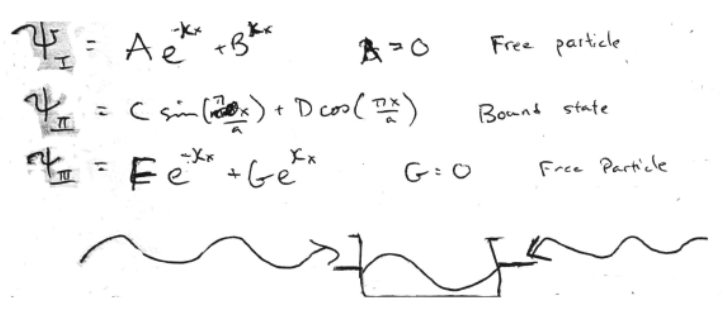

Figure 3.3 An incorrect sketch of a wavefunction with bound and scattering states simultaneously.

We have administered a multiple-choice question to 85 students asking them to judge whether the energy levels $E_{1}$ and $E_{2}$ correspond to a particle in a bound state or a scattering state in a finite square well as shown in Figure 3.4. Nineteen percent of the students incorrectly believed that a single particle in a given stationary state can have energy $E_{1}$ inside the well and have a different energy $E_{2}$ outside the well. These students failed to notice that one stationary state wavefunction cannot have different energies in different regions. Therefore, a quantum particle in a given state cannot have a bound state wavefunction in some regions and a scattering state wavefunction in other regions.

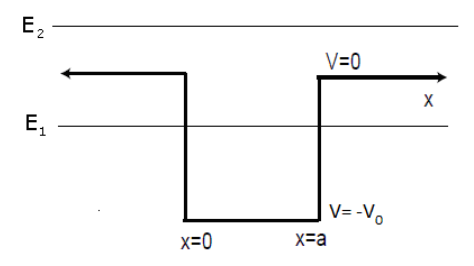

Figure $3.4 E_{1}$ corresponds to a bound state and $E_{2}$ corresponds to a scattering state. A quantum particle in a stationary state cannot have different energies in different regions. 


\subsubsection{Difficulties related to the quantum tunneling effect}

In quantum mechanics, there is a non-zero probability for a particle to be found in the classically forbidden regions, which is usually called the tunneling effect. However, some students incorrectly believe that the tunneling effect can only exist in the scattering states. For example, we asked students whether a quantum particle with energy $E$ interacting with a piecewise continuous potential energy as shown in Figure 3.5 is in a bound state or a scattering state. Since $E<V$ at both plus and minus infinity, the particle should be in a bound state. But some students claimed that the particle is in a scattering state because "it can tunnel through the barriers". Some other students believed that the particle is in a bound state in the regions (II), (III) and (V) but in a scattering state in the regions (I), (IV) and (VI) since "classically the particle cannot be found in those regions".

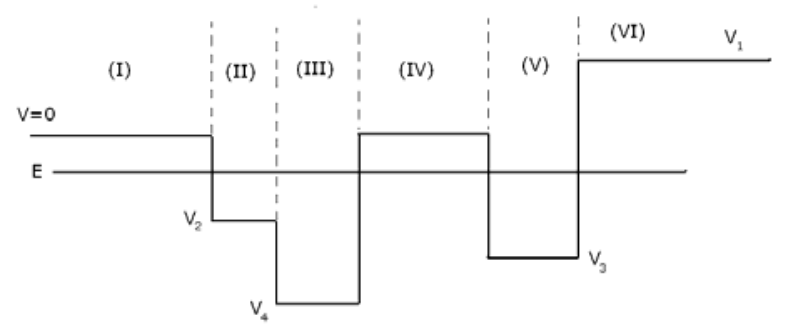

Figure 3.5 An example of a bound state particle tunneling into the classical forbidden regions.

Moreover, although some students realized that a non-zero wavefunction could exist in the classically forbidden regions, they still mistakenly believed that it is impossible to find the particle in the regions where the energy of the particle is lower than the potential energy $(E<V)$. When we asked the students where they can find the particle for a given potential energy well as shown in Figure 3.5, several students claimed that the particle cannot be found in regions (I), (IV) and (VI) because "the particle only tunneled through these barriers". However, when the students 
were explicitly asked about whether a bound state particle can be found outside the finite square well, they would answer yes with no doubt.

\subsubsection{Difficulties in determining bound states related to the maximum value of potential energy vs. the energy of the particle}

A particle is in a quantum mechanical bound state if and only if its energy is less than the potential energy at both plus and minus infinity. However, some students are confused about the state of the particle when its energy is lower than the maximum value of the potential energy. We have administered a question to 15 students asking them about the state of a particle with energy $E$ interacting with a potential energy barrier (with maximum value $V_{0}$ ) as shown in Figure 3.6. Only seven out of the fifteen students correctly answered that the particle is in a scattering state. In another multiple-choice question about the same system (in Figure 3.6) administered to 85 students, $19 \%$ of the students chose the option that the particle is in a bound state when $E<V_{0}$ and in a scattering state when $E>V_{0}$.

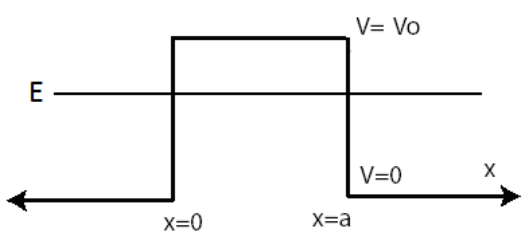

Figure 3.6 A scattering state particle with energy $E$ lower than the potential energy barrier $V_{0}$. 


\subsubsection{Difficulties related to the directional preference of the scattering state wavefunctions for a symmetric potential energy}

A scattering state wavefunction is an energy eigenfunction with energy $E$ higher than the potential energy at either plus or minus infinity or both. A scattering state wavefunction could have directional preference, e.g., a particle can be launched from the left hand side of a 1D finite square well. However, the directional preference is not necessary and a scattering state wavefunction can have no directional preference. For example, $e^{i k x}$ and $e^{-i k x}$ are scattering state wavefunctions for a 1D free particle system with opposite wave vectors $\vec{k}$ and $-\vec{k}$ but the same energy proportional to $|\vec{k}|^{2}$. So their superposition wavefunction $e^{i k x}+e^{-i k x}$ is still an energy eigenfunction with the same energy as the scattering state wavefunctions $e^{i k x}$ and $e^{-i k x}$. But there is no directional preference for the scattering state wavefunction $e^{i k x}+e^{-i k x}$, which is a standing wave.

However, many students have difficulties with the issues related to the directional preference of a scattering state wavefunction. Some students incorrectly assumed that all scattering states must correspond to a particle being launched from minus or plus infinity. Such misconception could be due to over-generalizing their experience about the experiments of scattered particles in modern physics. While some other students claimed that the scattering state wavefunctions for a finite square well can be symmetric, their reasoning was incorrect. For example, some of them incorrectly sketched the scattering state wavefunction as a symmetrical straight line. 


\subsubsection{Difficulties related to the transmission and reflection of a quantum mechanical particle in the scattering state}

Classically, when a particle with energy $E$ moves toward a region with higher potential energy $V_{0}>E$, the particle will be bounced back. Or if the potential energy is lower, i.e., $V_{0}<E$, the particle will transmit without reflection. However, in quantum mechanics, the wavefunction in general has a non-zero probability of transmission and reflection simultaneously. For a particle in a scattering state being launched from the left side of a 1D finite square well, it could have a nonzero probability of being bounced back by the well. Thus, the wavefunction on the left side of the well has components of both incident and reflected waves, e.g., $A e^{i k x}+B e^{-i k x}$.

Some students have difficulties with the transmission and reflection of a quantum mechanical particle in the scattering states in quantum mechanics. A multiple-choice question about a particle launched from minus infinity with energy $E>0$ interacting with a $1 \mathrm{D}$ finite square well (with the depth $-V_{0}<0$ as shown in Figure 3.7) was administered to 18 students. Only $67 \%$ of the students correctly selected the options that the particle not only has a non-zero probability of passing through the well but also a non-zero probability of being bounced back.

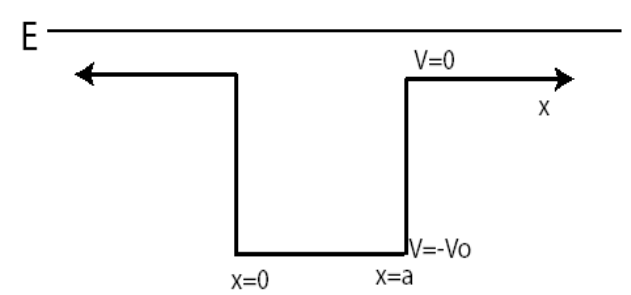

Figure 3.7 The particle launched from the left hand side of the potential energy well has a non-zero probability of being bounced back by the potential energy well. 


\subsection{RESEARCH-BASED LEARNING TUTORIAL FOR BOUND \& SCATTERING STATE WAVEFUNCTION}

Based on the investigation of students' difficulties, we developed research-based learning tools such as a QuILT and peer instruction tools to improve students' understanding of the bound and scattering state wavefunctions. The QuILT helps the students learn about the bound and scattering state wavefunctions using a guided approach. We also used computer-based visualization tools in the QuILT to help students develop physical intuition about the bound and scattering state wavefunctions for different potential energies. The simulations we adapted in the QuILT on bound and scattering state wavefunction were developed by the PhET team at the University of Colorado (McKagan et al, 2009). Before the students start using the QuILT, a warm-up tutorial is provided to prepare all students with the necessary preliminary knowledge.

The peer instruction tools address students' common difficulties related to the bound and scattering state wavefunctions so that the students could reduce some misconceptions and build a better knowledge structure through peer discussion. We have used the concept tests as our peerinstruction tools in the junior-senior level quantum mechanics course at the University of Pittsburgh for two years. Students must discuss each concept test question with their peers before they submit the answers through the clickers (electronic system). The instructors discuss the concepts and principles involved in the concept test questions if the students show difficulty in answering the questions. 


\subsubsection{Warm-up tutorial for the bound \& scattering state wavefunction}

In the warm-up tutorial, students get an opportunity to review how to derive the energy eigenfunctions from the TISE and why the energy eigenstates can be categorized into bound states and scattering states. Students are asked to write the TISE as

$$
\frac{\hbar^{2}}{2 m} \frac{\partial^{2} \Psi}{\partial x^{2}}=(V(x)-E) \Psi,
$$

where $V(x)$ is the potential energy and $E$ is the energy eigenvalue. When the potential energy is greater than the energy of the particle, the curvature of the wavefunction $\partial^{2} \Psi / \partial x^{2}$ has the same sign as the wavefunction $\Psi$. Students are asked to sketch a function such that the curvature of the wavefunction and the value of the wavefunction are both positive (or both negative) everywhere. Through plotting the function, the students will realize that such a function will keep increasing while approaching infinity (or keep decreasing while approaching minus infinity) so it cannot be a normalizable wavefunction. Therefore, students could learn why the energy of a quantum system must be greater than the minimum value of the potential energy.

Students are also required to qualitatively draw a wavefunction in the region near infinity based on the relation between the curvature and the value of the wavefunction (Eq 3.3). They find that the wavefunction at plus/minus infinity is oscillatory if $E>V( \pm \infty)$ and decays to zero if $E<V( \pm \infty)$. A multiple-choice question helps students learn that when the energy is less than the potential energy at both plus and minus infinity, the wavefunction can be considered as bounded in a finite range because the probability of finding the particle at plus and minus infinity is zero. Thus, the students are guided to understand that whether a quantum system is in a bound state or a scattering state depends on the energy of the quantum particle compared to the potential energy value at plus/minus infinity. 
After the students learn that the bound states and the scattering states are both energy eigenstates, the QuILT asks them whether a quantum particle could have different energy in different regions of a piecewise continuous potential energy well (e.g., Figure 3.8). The QuILT also contains a dialogue question to elaborate that a particle in an energy eigenstate can only have one energy while its wave function can extend over various regions and the energy of the particle is not well defined if the wavefunction is not an energy eigenstate. The warm-up questions also help students learn that the energy spectrum of a quantum system is discrete for the bound states and continuous for the scattering states.

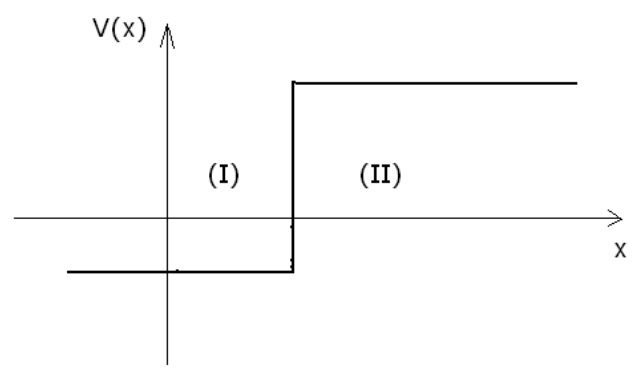

Figure 3.8 An energy eigenstate only corresponds to one energy though the potential energy could have different values in different regions.

\subsubsection{QuILT on the bound \& scattering state wavefunction}

At the beginning of the QuILT, we ask the students a sequence of questions to help them distinguish between the classical bound states and the quantum bound states. Then students are given some questions about the $1 \mathrm{D}$ infinite square well and the simple harmonic oscillator (SHO) potential energy well which only allow bound states. Students also predict whether a quantum particle can be found in the classically forbidden regions of a SHO potential energy well and then they can use the simulation to observe the bound state wavefunctions for a SHO and reconcile the differences between their predictions and observations. They also compare and 
explain the similarities and differences between the bound state wavefunctions of a $1 \mathrm{D}$ infinite square well and a SHO.

After the students have learned the simple models, e.g., the 1D infinite square well or the SHO, which only allow bound states, they are guided with some targeted questions about the 1D finite square potential energy well $\left(V(x)=-V_{0}<0\right.$ between $0 \leq x \leq a$ and $V(x)=0$ elsewhere) that allows both bound and scattering states. Students first predict the shape of a bound state wavefunction inside and outside the finite square well when the energy is less than zero. A computer simulation helps the students observe the shape of the bound state wavefunctions for a finite square well so that they can examine their prediction and reconcile any differences between the prediction and observation. Then, the students are asked to consider whether a scattering state wavefunction with energy $E>0$ incident from the left side of the well will necessarily be bounced back by the well. In the simulation about the scattering state wavefunctions, students can watch that the amplitude of the wavefunction is a constant on the right hand side of the well but not on the left hand side. Students learn that the changing amplitude of wavefunction on the left hand side is due to the interaction between the incident wave and the reflected wave. In a pictorial multiple-choice question as shown in Figure 3.9, students are asked to select the correct shape of a scattering state wavefunction with no directional preference (e.g., option (II) in Figure 3.9) when the particle is not sent from one side of the well. A dialogue question in the QuILT helps students understand why a scattering state wavefunction does not necessarily have directional preference. 


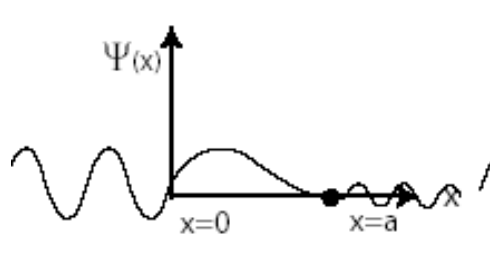

(I)

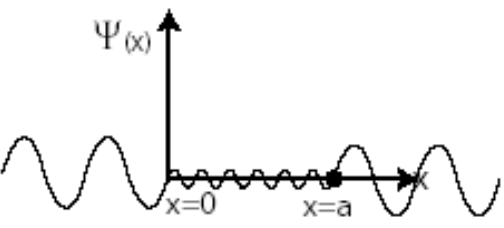

(II)

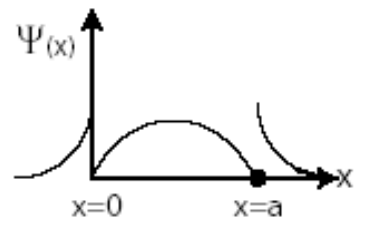

(III)

Figure 3.9 A multiple choice question asking students to choose the correct qualitative sketch of a scattering state wavefunction with no directional preference. The correct answer is (II) only.

Some potential energies such as a 1D finite square barrier $\left(V(x)=V_{0}>0\right.$ between $0 \leq x \leq a$ and $V(x)=0$ elsewhere) only allow scattering state wavefunctions. As discussed in section 3.3.4, some students mistakenly believe that a wavefunction is in a bound state when the energy of the particle $E$ is less than the maximum potential energy $V_{0}$. To reduce these difficulties about the scattering states for a particle interacting with a 1D finite square potential energy barrier, the QuILT asks the students to predict the state (bound or scattering) of a quantum particle when its energy is higher or lower than $V_{0}$. Then the students could use the simulation to change the height of a potential barrier and the energy level of a particle to observe the shape of the wavefunction inside and outside the barrier. Students will find that the wavefunction is always oscillatory on the left and right sides of the barrier no matter whether the energy of the particle is lower or higher than the potential barrier. Therefore, for a particle interacting with a finite potential energy barrier, its energy eigenfunctions always correspond to scattering states. Students are also asked to predict whether the wavefunction will be reflected by the barrier or transmit through the barrier. Their predictions can be checked via the simulation by comparing the amplitude of the wavefunction on the left and right sides of the barrier. The varying amplitude of probability density on one side of the well indicates that the incident 
wavefunction is reflected by the potential energy barrier. A snap shot of the simulation is shown in Figure 3.10.

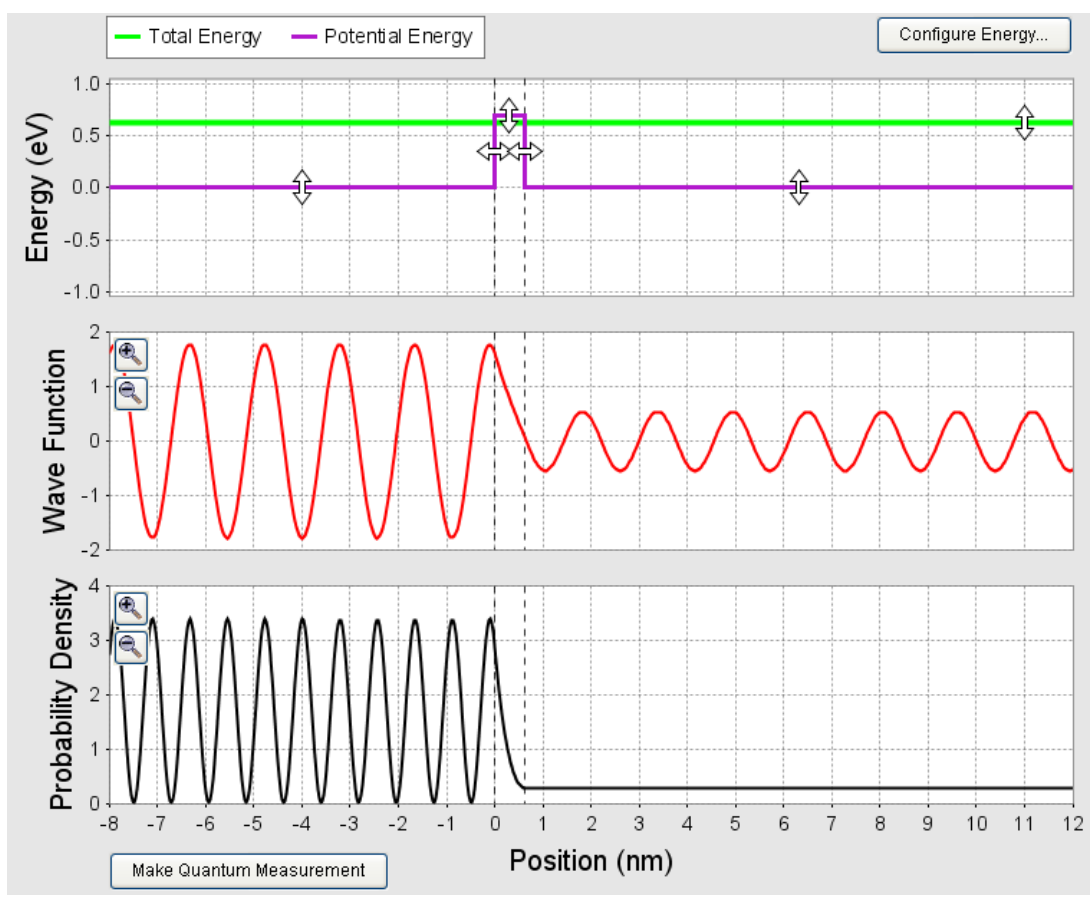

Figure 3.10 PhET simulation of a scattering state wavefunction incident from the left hand side of a potential energy barrier. The probability density (square of the amplitude) of the wavefunction varies on the left side of the well due to the interaction between the incident and reflected wavefunctions.

In addition to the QuILT, a sequence of research-based concept test questions can be integrated with the lectures as peer-instruction tools to improve students' understanding of the issues related to bound and scattering state wavefunctions. The concept test questions will be discussed in chapter 8 in this thesis.

\subsection{PRELIMINARY EVALUATION}

We conducted preliminary evaluation of the QuILT about bound and scattering state wavefunctions in two junior-senior level quantum mechanics classes, the first with 14 students 
and the second with 18 students. Students first received lectures and then used the concept tests as peer-instruction tools in both classes when learning the topics related to bound and scattering state wavefunctions. After the lecture and the peer instruction in class, students took the pre-test and then worked on the QuILT. The post-test was administered in the following class period after students had finished the QuILT. To eliminate any possible differences in the difficulty levels of the pre-test and the post-test, we designed two versions of a test, i.e., Test A and Test B, each of which has six questions. Students were randomly given either Test A or Test B as the pre-test and then each of them was administered the version of the test he/she had not taken as the post-test. In particular, 18 students in the two classes were administered Test $\mathrm{A}$ as the pre-test and 17 of them (one student absent) took Test B as the post-test. The other 14 students were given Test B as the pre-test and 12 of them (two students absent) took Test A as the post-test. The average pre-test score for all 32 students was $69 \%$ and the average post-test score for the 29 students was $86 \%$. The average pre-test and post-test score on each question combining the two classes is listed in Table 3.1.

Questions A-1 and B-1 require the students to consider a particle with energy $E$ interacting with a piecewise continuous potential energy as shown in Figure 3.5 (question B-1) and Figure 3.11 (question A-1). Students are asked to write down all the possible regions where a classical particle or a quantum particle could be found. The improved performance suggests that the students are more likely to understand that a quantum particle in either a bound or scattering state could be found in the classical forbidden regions. Questions A-3 and B-4 ask the students whether a quantum particle with energy $E$ incident from $x=-\infty$ would be bounced back by a finite square well (question A-3 as shown in Figure 3.7) or a finite square barrier (question B-4 as shown in Figure 3.12). The results of questions A-3 and B-4 indicate that students have a 
better understanding of the fact that an incident particle has a finite probability of reflection even if its energy is higher than the maximum value of the potential energy well/barrier. A statement about the directional preference of the scattering state for a finite square well is used in both questions A-6 and B-6 as shown below. The instructor did not explain the pre-test questions to the students before they took the post-test and the students did not know that such questions would be asked again in their post-test. In the post-test, students showed better understanding of why the scattering state wavefunction for a finite square well can be symmetric without directional preference. They also sketched clearer graphs of wavefunctions to support their reasoning.

Question A-6/B-6: Student A says that the scattering state wavefunction for an electron interacting with a finite square well can never be symmetric about the center of the well because the electron has to be launched from either left or right. Student B says that the scattering state wavefunction for a finite square well can be symmetric about the center of the well because the electron need not be launched from left or right. Explain why you agree or disagree with each student. Qualitatively sketch a wavefunction to support your answer.

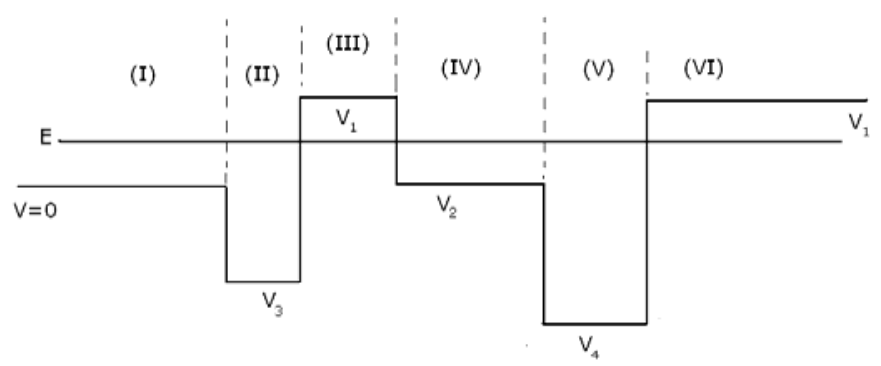

Figure 3.11 A particle with energy $E$ interacting with a piecewise continuous potential energy well. 


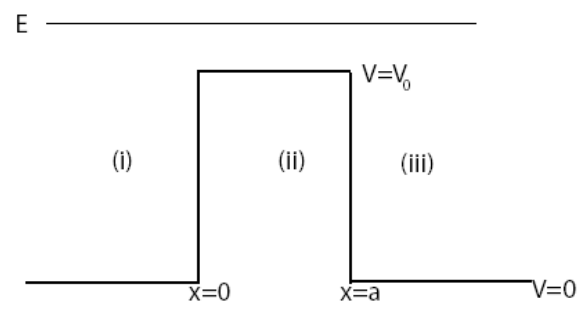

Figure 3.12 A particle with energy $E$ incident from the left side of a finite square barrier.

Table 3.1 The pre-test and post-test scores on each question. A and B represent the test version.

\begin{tabular}{|c|c|c|c|c|c|c|c|c|c|c|c|c|}
\hline Question & A-1 & A-2 & A-3 & A-4 & A-5 & A-6 & B-1 & B-2 & B-3 & B-4 & B-5 & B-6 \\
\hline Pre-test & $75 \%$ & $94 \%$ & $67 \%$ & $89 \%$ & $64 \%$ & $56 \%$ & $68 \%$ & $61 \%$ & $93 \%$ & $50 \%$ & $57 \%$ & $43 \%$ \\
\hline Post-test & $96 \%$ & $92 \%$ & $92 \%$ & $83 \%$ & $67 \%$ & $79 \%$ & $82 \%$ & $97 \%$ & $100 \%$ & $76 \%$ & $76 \%$ & $91 \%$ \\
\hline
\end{tabular}

Since the peer-instruction tools used in class before the pre-test may enhance students' performance in the pre-test, we administered the pre-test of bound and scattering state wavefunctions to another group of students (the comparison group) who had only received traditional instruction without using the concept tests and the QuILT. In the comparison group, 15 students took Test A and 10 students took Test B after traditional instruction. The average score for all the 25 students in the comparison group is $41 \%$. The comparison group students' average score on each question is listed in Table 3.2. By comparing the students' pretest scores after using the peer-instruction tools (as shown in Table 3.1) with the comparison group's scores (as shown in Table 3.2), we can infer that the concept test reduced students' difficulties in some issues related to bound and scattering state wavefunctions. For example, the multiple-choice question B-3 asks the students whether a 1D finite square well allows bound states or scattering states. In the comparison group, only $50 \%$ of the students knew that both bound and scattering states could exist for a 1D finite square well and many students mistakenly believed that only 
bound states were allowed. Question A-4 requires the students to judge the state of a particle with energy $E$ interacting with a higher potential energy barrier as shown in Figure 3.6. More than half of the comparison group students incorrectly believed that the particle is in a bound state when the energy is lower than the maximum value of potential energy. In question A-2 we asked the students to sketch the energy levels of the bound states and scattering states for a $1 \mathrm{D}$ finite square well. The comparison group students had difficulties in realizing that the energy of the particle cannot be lower than the minimum value of the potential energy. Also some students in the comparison group did not understand that the scattering states are energy eigenstates with definite energy value. A student sketched a classical scattering situation such that an incident particle was reflected when hitting the potential energy well (as shown in Figure 3.13). Another student claimed that the scattering state requires a potential energy barrier instead of a potential energy well and sketched a plot as shown in Figure 3.14.

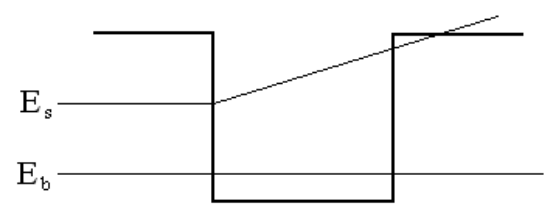

Figure 3.13 A student in the comparison group incorrectly sketched a classical scattering situation to represent the energy level for a quantum scattering state.

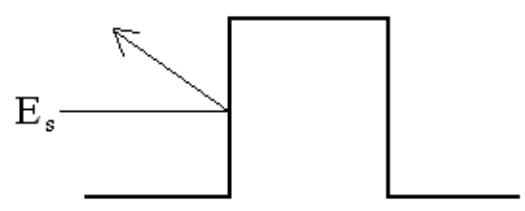

Figure 3.14 A student in the comparison group believed only the energy barrier allows a scattering state and he sketched a classical scattering situation to represent the energy level for the quantum scattering state. 
Table 3.2 The control group scores on each question. A and B represent the test version.

\begin{tabular}{|c|c|c|c|c|c|c|c|c|c|c|c|c|}
\hline Question & A-1 & A-2 & A-3 & A-4 & A-5 & A-6 & B-1 & B-2 & B-3 & B-4 & B-5 & B-6 \\
\hline After Lecture & $60 \%$ & $57 \%$ & $7 \%$ & $47 \%$ & $50 \%$ & $23 \%$ & $55 \%$ & $55 \%$ & $50 \%$ & $10 \%$ & $50 \%$ & $25 \%$ \\
\hline
\end{tabular}

\subsection{CONCLUSION}

We have investigated students' difficulties related to bound and scattering state wavefunctions for different quantum systems and used the findings as a guide to develop the QuILT related to bound and scattering state wavefunctions. Preliminary evaluation suggests that the QuILT about bound and scattering state wavefunctions is effective in improving students' understanding of these concepts. Comparison between the class using peer-instruction tools together with the lectures and the class having only traditional lectures suggests that the peer-instruction tools can reduce students' difficulties about bound and scattering state wavefunctions.

\subsection{CHAPTER REFERENCE}

Chi, M. (1994). “Thinking Aloud”, M. Van Someren, Y. Barnard, and J. Sandberg (eds.), The Think Aloud Method: A Practical Guide to Modeling Cognitive Processes. Edited by London: Academic.

McKagan, S., Malley C., Adams, W., Perkins, K. and Wieman, C. (2009). Simulation program is available at $<$ http://phet.colorado.edu $>$ 


\title{
4.0 IMPROVING STUDENTS’ UNDERSTANDING OF DRAWING ENERGY EIGENFUNCTIONS
}

\begin{abstract}
4.1 ABSTRACT
In this chapter, we describe the difficulties students have with drawing the energy eigenfunctions. We also discuss the development and implementation of a research-based Quantum Interactive Learning Tutorial (QuILT) to reduce these difficulties. The preliminary evaluation shows that the QuILT about drawing the energy eigenfunctions is effective in improving students' understanding of the concepts related to the shape of the energy eigenfunctions.
\end{abstract}

\subsection{BACKGROUND}

As stated by the correspondence principle, when the energy of a quantum system is high enough, the behavior of the quantum system is close to the corresponding classical system. Therefore, we can use a semi-classical approach to qualitatively analyze the shape of the energy eigenfunctions with high energy levels. In the semi-classical approximation, the kinetic energy $K$ for a particle in a quantum system is given by $K=E-V(x)$ where $E$ is the energy eigenvalue (total energy) and $V(x)$ is the potential energy of the system. The shape of the energy eigenfunction depends 
on the value of the kinetic energy $K$. In the regions where the kinetic energy $K$ is negative ( $K<0$ ), the energy eigenfunction decays as $e^{-k x}$ in which the magnitude of the wave vector $k$ is defined by

$$
k=\frac{\sqrt{2 m|K|}}{\hbar} \text { ( } m \text { is the mass of the particle and } \hbar \text { is reduced Planck's constant). (Eq 4.2) }
$$

In the regions where the kinetic energy $K$ is positive $(K>0)$, the energy eigenfunction is oscillatory. In order to qualitatively sketch the oscillatory energy eigenfunction, we need to know the wavelength of the wavefunction. The wavelength of an energy eigenfunction can be expressed by the de Broglie relation $\lambda=2 \pi \hbar / p$ where the magnitude of the momentum $p$ for the quantum system is proportional to the magnitude of the wave vector $k$, i.e.,

$$
p=\hbar k=\sqrt{2 m K} .
$$

Therefore, the higher the kinetic energy $K$, the shorter the wavelength $\lambda$.

If the kinetic energy $K$ is positive, the relative amplitude of the oscillatory energy eigenfunction in different regions can also be determined by the semi-classical approximation. In the regions where the kinetic energy of the particle is larger, the momentum of the particle is larger so the particle moves "faster". Therefore, the particle will spend less time in the region with higher kinetic energy $K$ and the probability of finding the particle in that region is lower. Since the absolute square of the amplitude of the wavefunction $|\psi(x)|^{2}$ represents the probability density of finding the particle at the position $x$, the absolute value of the amplitude of the wavefunction $|\psi(x)|$ must be lower in the region where the kinetic energy is higher. 


\subsection{INVESTIGATION OF STUDENT'S DIFFICULTIES}

In these investigations, our goal was to examine students' difficulties with drawing the energy eigenfunctions after traditional instruction so that we can devise strategies to improve students' understanding. The investigation of students' difficulties with drawing the energy eigenfunctions was carried out by administering written surveys to many advanced undergraduate and graduate students enrolled in quantum mechanics courses and by conducting individual interviews with a subset of them. We used open-ended questions to probe students' difficulties by explicitly asking them to sketch the energy eigenfunctions for a given quantum system. The individual interviews were conducted using a think-aloud protocol (Chi 1994). In the think-aloud interviews, students were asked to verbalize their reasoning process and not interrupted unless they remained silent for a while. At the end of the interview, they were asked to explain the issues which they had not clarified earlier.

\subsubsection{Difficulties related to the position of the $\psi(x)=0$ axis}

If the total energy $E$ of the particle is higher than the potential energy $V(x)$, the energy eigenfunction is oscillatory about the $x$-axis of the $\psi(x)$ vs. $x$ plot. On the other hand, if the total energy $E$ is less than the potential energy $V(x)$ at plus/minus infinity, the energy eigenfunction decays to $\psi(x)=0$ at plus/minus infinity. However, some students have difficulties in distinguishing between the vertical axis of the potential well (which has the units of energy) with the vertical axis of the wavefunction so they may shift the position of the $\psi(x)=0$ axis when sketching the energy eigenfunctions. For example, in a survey question, 
students were given a potential energy diagram for a one-dimensional (1D) finite square well of width $a$ and depth $-V_{0}$ between $0 \leq x \leq a$. Students were asked to qualitatively sketch an energy eigenfunction with positive energy $E>0$. A student incorrectly drew the wavefunction to be higher in the middle of the well as shown in Figure 4.1 and claimed that "(the wavefunction is) higher because some of the wave is reflected at the wall”. Similar mistakes also appeared when we asked the students to draw an energy eigenfunction with negative energy $E<0$ for a 1D finite square potential energy well. The wavefunction should oscillate about the axis $\psi(x)=0$ inside the well and decay to zero at plus/minus infinity outside the well. However, some students shifted the value of $\psi(x)$ about which it oscillates to $\psi(x)>0$ inside the well even though they had noticed that the decaying axis outside the well was $\psi(x)=0$.

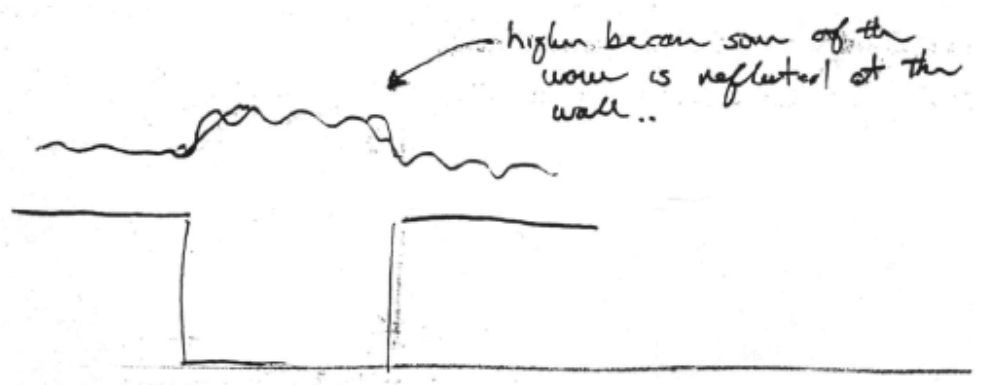

Figure 4.1 An incorrect sketch of an energy eigenfunction that oscillates about different axis.

\subsubsection{Difficulties related to decaying and oscillatory wavefunctions}

The energy eigenfunction should be oscillatory in the regions where $E>V(x)$ and decaying in the regions where $E<V(x)$. However, some students have difficulties in determining whether the energy eigenfunction is decaying or oscillatory in a given region. When we asked the students to draw an energy eigenfunction of a particle with energy $E>0$ interacting with a 1D 
finite square well of width $a$ and depth $-V_{0}$ between $0 \leq x \leq a$, some students incorrectly sketched decaying wavefunctions inside the well as shown in Figure 4.2. These students have not learned what one should observe when the potential energy $V(x)$ is lower in the well. Instead, they plotted a decaying wavefunction from rote memory that may correspond to a particle interacting with a potential energy barrier. Moreover, similar to the student's plot in Figure 4.1, the student who sketched Figure 4.2(a) incorrectly claimed that "(the wavefunction is a) typical particle wavefunction but lowered by potential well” as though the oscillations on different sides of the well should be around different references.

(a)

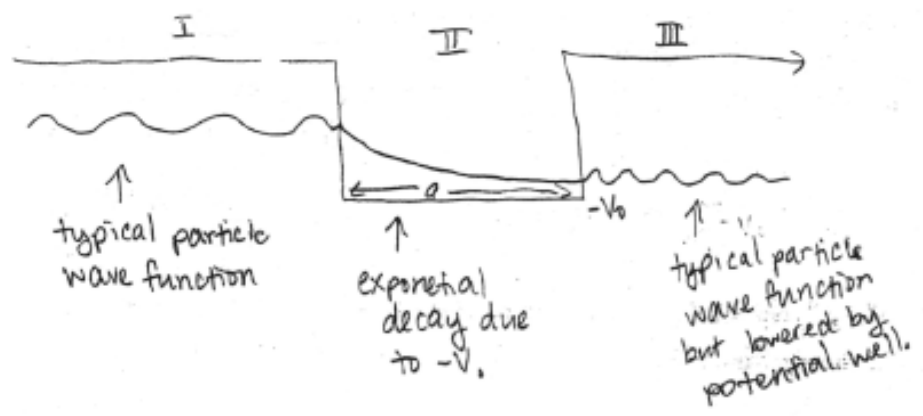

(b)

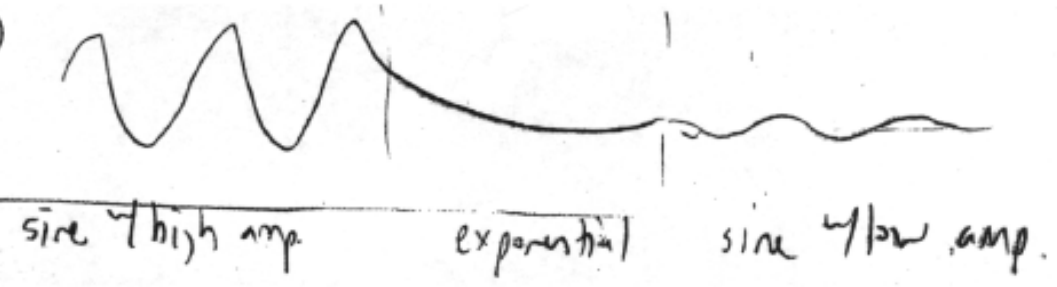

Figure 4.2 Incorrect sketches of an energy eigenfunction in a finite square well.

For an energy eigenfunction with an energy eigenvalue $E<V(x)$, the higher is the potential energy, the faster the wavefunction decays. We asked 12 undergraduate students in a junior-senior level quantum mechanics course to sketch the energy eigenfunction for a particle with energy $E$ interacting with a piecewise continuous potential energy well as shown in Figure 4.3. Only half of the 12 students correctly noticed that the wavefunction should decay faster in region (III) than in region (I) since the potential energy is higher in region (III). 


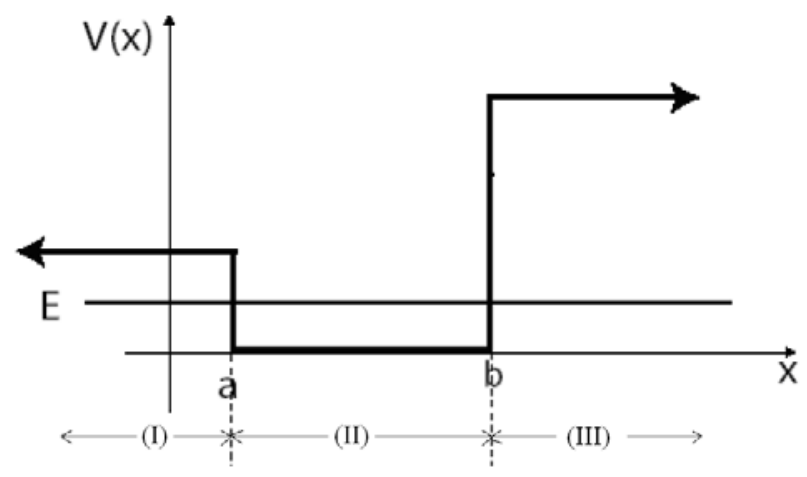

Figure 4.3 A particle with energy E interacting with a piecewise continuous potential energy well. The energy eigenfunction should decay faster in region (III) than in region (I).

Students also have difficulties with drawing the energy eigenfunction for a potential energy well with infinite potential energy on one side of the well. For example, when we asked the students to draw an energy eigenfunction for a half simple harmonic oscillator potential energy well $\left(V(x)=A x^{2}\right.$ for $x \leq 0$ and $V(x)=+\infty$ for $x>0, A$ is a positive constant), some students sketched non-zero wavefunctions in the region $x>0$ where the potential energy is infinity. These students did not realize that a quantum particle cannot be found in the regions where $V(x)=+\infty$ and the wavefunction in these regions must be zero.

\subsubsection{Difficulties related to the wavelength of oscillatory wavefunctions}

As discussed in section 4.2, for an oscillatory energy eigenfunction with energy $E>V(x)$, the wavelength of the wavefunction is shorter in the regions where the kinetic energy $K=E-V(x)$ is larger. We asked 12 students to explicitly comment on the wavelength of the energy eigenfunction for a particle with energy $E>0$ interacting with a 1D finite square well of width $a$ and depth $-V_{0}$ between $0 \leq x \leq a$ as shown in Figure 4.4. Only 3 students correctly drew an oscillatory wavefunction with longer wavelength in regions (I) and (III) than in region (II). Four 
students sketched an oscillatory wavefunction with the same wavelength in all regions and other students mistakenly drew a decaying wavefunction in regions (I) and (III).

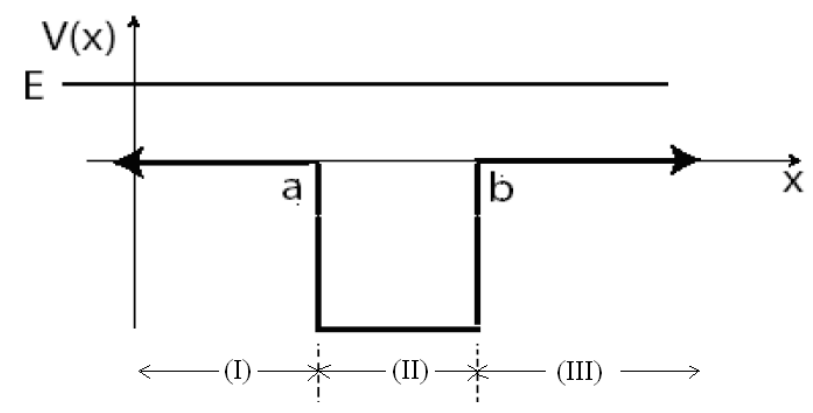

Figure 4.4 A particle with energy $E>0$ interacting with a finite square well. The wavelength of the oscillatory energy eigenfunction is longer in regions (I) and (III) than in region (II).

\subsubsection{Difficulties related to the absolute value of the amplitude of wavefunctions}

Students have great difficulties in understanding the relation between the amplitude of the energy eigenfunction and the potential energy in different regions. We asked seventeen students to sketch the energy eigenfunction for a particle with energy $E>V_{0}$ interacting with a $1 \mathrm{D}$ finite square barrier of width $a$ and height $V_{0}$ between $0 \leq x \leq a$ as shown in Figure 4.5. Since the kinetic energy of the particle $K=E-V(x)$ is lower in region (II) than in regions (I) and (III), the probability of finding the particle should be higher in region (II) than in regions (I) and (III). Therefore, the absolute value of the amplitude of the energy eigenfunction is larger in region (II). However, only one out of the seventeen students correctly sketched a wavefunction with higher absolute value of amplitude in region (II). Two students incorrectly sketched a wavefunction with lower absolute value of amplitude in region (II) and another two students mistakenly drew a decaying wavefunction in region (II). The other students did not notice that the absolute value of 
the amplitude of the energy eigenfunction should vary in different regions when they were drawing the plots.

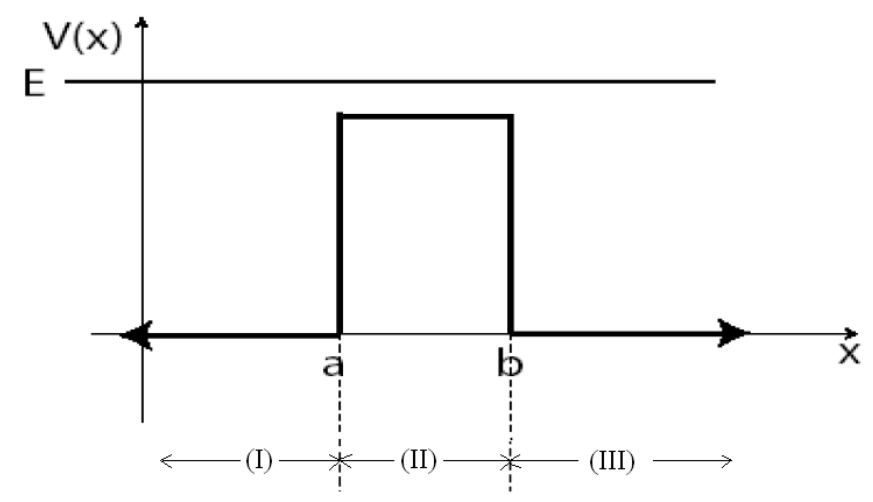

Figure 4.5 A particle with energy $E$ interacting with a finite square barrier. The absolute value of the amplitude of the energy eigenfunction is larger in region (II) than in regions (I) and (III).

Students also have difficulties with the issues related to the shape of the energy eigenfunction with directional preference. If a quantum particle is sent from the left hand side $(x \rightarrow-\infty)$ of a potential energy well (or barrier), its wavefunction has a finite probability of being bounced back. Therefore, the absolute value of the amplitude of the wavefunction on the left hand side of the well (a superposition of incident and reflected wavefunctions) is larger than the absolute value of the amplitude of the wavefunction on the right hand side of the well (transmitted wavefunction only). We asked twelve students to sketch the energy eigenfunction of a particle with energy $E>V_{0}$ incident from $x \rightarrow-\infty$ interacting with a potential energy barrier of height $V_{0}$. None of the twelve students expressed the difference between the absolute values of the amplitude of the wavefunction on the left and right sides of the potential energy barrier though we explicitly told them that the particle was sent from one side $(x \rightarrow-\infty)$. 


\subsection{RESEARCH-BASED LEARNING TUTORIAL FOR DRAWING ENERGY EIGENFUNCTIONS}

Based on the investigation of students' difficulties, we developed the research-based QuILT to improve students' understanding of drawing energy eigenfunctions. The QuILT helps the students learn to sketch the energy eigenfunction through a guided approach. We also incorporated computer-based visualization tools in the QuILT to help students observe the shape of the energy eigenfunctions for different quantum systems. The simulations we adapted in the QuILT on drawing energy eigenfunctions are developed by the PhET team at the University of Colorado (McKagan et al, 2009).

At the beginning of the QuILT, students need to consider a particle with energy $E$ interacting with a 1D finite square well as shown in Figure 4.6. The energy eigenfunction for this system is oscillatory in all the regions (I), (II) and (III). A sequence of questions asks the students to rank the magnitude of momentum $p$ in regions (I), (II) and (III) by comparing the kinetic energy $K$ of the particle in different regions. Based on the values of $p$ in different regions, students can use the de Broglie relation to compare the wavelength of the energy eigenfunction in regions (I), (II) and (III). Then a question in the QuILT explains to the students how to use a semi-classical approach to compare the absolute value of the amplitude of the wavefunction in different regions.

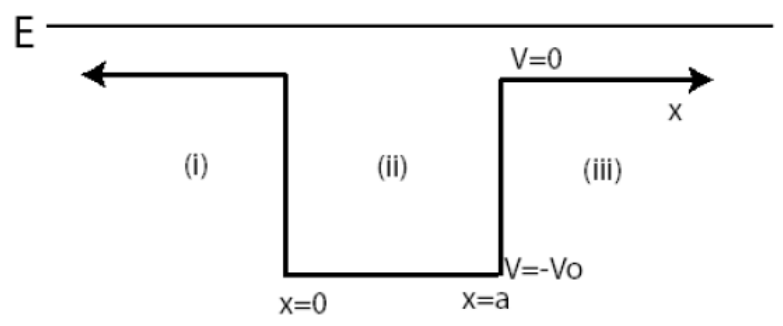

Figure 4.6 A quantum particle interacting with a 1D potential energy well. 
After the students have learned how to determine the wavelength and amplitude of the oscillatory energy eigenfunctions, they are asked to sketch the energy eigenfunction of a particle interacting with a 1D finite square well (Figure 4.6). A separate space is provided so that the students do not confuse the vertical axis in the potential energy diagram with the vertical axis of the wavefunction. Students need to compare the wavefunction with no directional preference and the wavefunction for a particle incident from one side of the well. The simulation in the QuILT of drawing energy eigenfunctions can help the students observe the shape of the energy eigenfunction for a particle incident from the left or right side of a $1 \mathrm{D}$ finite square well. Students can adjust the energy level of the incident particle and the depth of the 1D finite square well so they can find out how the wavelength and amplitude of the wavefunction vary with the different values of $E-V$ ( $E$ is the total energy and $V$ is the potential energy). A question in the QuILT shows the students the correct shape of a symmetric energy eigenfunction with no directional preference and another question explains to the students that the energy eigenfunction oscillates about the axis $\psi(x)=0$ when $E>V$. By sketching the wavefunction of a particle with energy $E<0$ interacting with a 1D finite square well, students learn that the energy eigenfunction decays to zero at plus or minus infinity if the total energy $E$ is less than the potential energy $V$ at infinity.

The QuILT also asks the students to consider the wavelength and the amplitude of the energy eigenfunction for a particle interacting with a 1D finite square barrier as shown in Figure 4.7. Students need to first predict the shape of the energy eigenfunction for a particle incident from the left hand side of the barrier and then use the simulation to check their predictions. They can find that the absolute value of the amplitude of the wavefunction is larger on the left hand side of the well than on the right hand side. Students are asked to adjust the height of the 
potential energy barrier $V_{0}$ and the energy level of the incident particle $E\left(E<V_{0}\right)$ to observe that the wavefunction decays faster inside the barrier when the difference between $V_{0}$ and $E$ increases. They can also find that the energy eigenfunction is oscillatory in all three regions if the energy of the particle is higher than the potential energy barrier $\left(E>V_{0}\right)$. A question in the QuILT shows the students a symmetric wavefunction for a particle interacting with the potential energy barrier to help students understand that energy eigenfunctions without directional preference are allowed in the quantum system as shown in Figure 4.7.

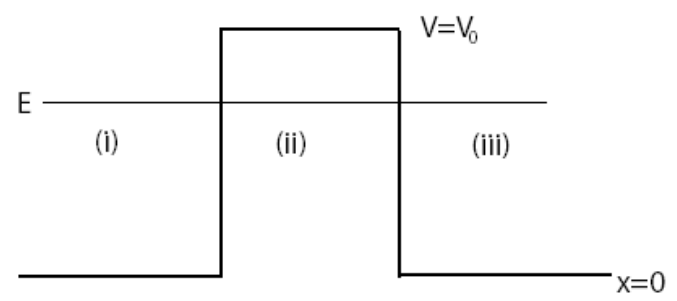

Figure 4.7 A quantum particle interacting with a 1D potential energy barrier.

At the end of the QuILT of drawing energy eigenfunctions, students are asked to solve a problem about a particle interacting with a triangular potential energy well as shown in Figure 4.8 where $V(x)=+\infty$ for $x<a$. We provide a guided approach in the QuILT to help students analyze the wavelength and the absolute value of the amplitude of an energy eigenfunction for the triangular potential energy well. Students first learn that the particle cannot be found in region (I) as shown in Figure 4.8 since the potential energy is infinite in that region. They also learn that the energy eigenfunction is oscillatory in region (II) and decays in region (III). Then students are asked to consider whether the kinetic energy of the particle increases or decreases as the position of the particle $x$ increases in the semi-classical approximation. Since the kinetic energy decreases as $x$ increases in region (II), the student can find that the wavelength and the absolute value of the amplitude of the energy eigenfunction both increase from $x=a$ to $x=b$. 
After analyzing the shape of the wavefunction, students need to qualitatively sketch the energy eigenfunction for the quantum system of the triangular potential energy well.

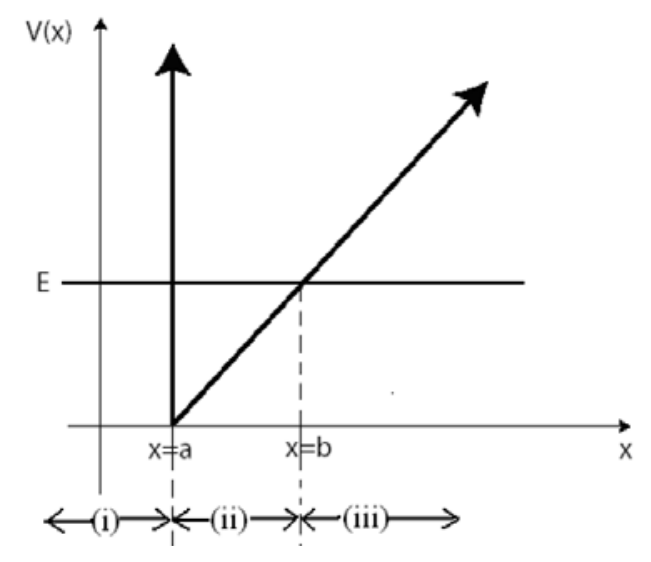

Figure 4.8 A quantum particle interacting with a triangular potential energy well.

\subsection{PRELIMINARY EVALUATION}

We conducted preliminary evaluation of the QuILT about drawing energy eigenfunctions in two junior-senior level quantum mechanics classes. Students first took the pre-test and then worked on the QuILT. The post-test was administered in the following class period after students had learned from the QuILT. To eliminate any possible differences in the difficulty levels of the pretest and the post-test, we designed two versions of a test, i.e., Test A and Test B, each of which has four open-ended questions. Students were administered either Test A or Test B as the pre-test and then each of them was given the version of the test he/she had not taken as the post-test. In particular, 17 students (one student absent) in the two classes were administered Test $\mathrm{A}$ as the pre-test and 18 students took Test B as the post-test. The other 12 students were given Test B as the pre-test and Test A as the post-test. The average pre-test score for 29 students is $34 \%$ and the average post-test score for 30 students is $89 \%$. 
Questions A-1 and B-1 required the students to consider a particle with energy $E$ interacting with a piecewise continuous potential energy as shown in Figure 4.3. In question B-1, the energy of the particle is lower than the potential energy in both the regions $x<a$ and $x>b$. In the pre-test, seven out of twelve students did not realize that the wavefunction should decay faster in the region $x>b$ than in the region $x<a$. In the post-test, 67\% students correctly sketched the energy eigenfunction decaying more rapidly in the region $x>b$. In question A-1, the energy of the particle $E$ is higher than the potential energy $V(x)$ in the region $x<a$ but still lower than the potential energy in the region $x>b$. So the energy eigenfunction decays only in region (III) $(x>b)$ and oscillates in regions (I) $(x<a)$ and (II) $(a<x<b)$. In the pre-test, eight out of seventeen students sketched the energy eigenfunctions with the correct oscillatory and decaying regions. Among these eight students, five of them correctly sketched a longer wavelength in region (I) than in region (II). Only one student noticed that the amplitude is higher in region (I) than in region (II). Students’ performance was much better in the post-test. Eleven out of twelve students knew the wavefunction is oscillatory in both regions (I) and (II). All of the eleven students correctly sketched the wavefunction with longer wavelength in region (I) and nine of them sketched higher absolute value of the amplitude of wavefunction in region (I).

Questions A-2 and B-3 asked the students to consider a particle with energy $E$ interacting with a potential energy barrier as shown in Figure 4.5. In question A-2, the particle is not launched from one side of the potential energy barrier so the energy eigenfunction is symmetric. In the pre-test, $41 \%$ of the students correctly sketched a symmetric wavefunction with longer wavelength in region (II) $(a<x<b)$ and only two out of the seventeen students knew the amplitude of the wavefunction is higher in region (II). In the post-test, 83\% of the 
students drew the energy eigenfunction with correct wavelength and $75 \%$ of the students sketched the wavefunction with correct amplitude. In question B-3, the particle is incident from the left side of the well so its energy eigenfunction is not symmetric. The amplitude on the left hand side of the well is higher than that on the right hand side. However, only one out of twelve students in the pre-test drew the wavefunction with correct amplitude and another one sketched the incident and reflected wavefunction on the left side of the well separately. In the post-test, $56 \%$ of the students correctly showed the directional preference in their plots. Questions A-3 and B-2 also asked the students about the energy eigenfunctions with or without directional preference. The model used in questions A-3 and B-2 is a particle interacting with a $1 \mathrm{D}$ square potential energy well as shown in Figure 4.4. Students' performance in questions A-3 and B-2 showed similar improvement as in questions A-2 and B-3 after they had learned the QuILT of drawing energy eigenfunctions.

Questions A-4 and B-4 tested students' understanding of sketching the energy eigenfunction for a triangular potential energy well as shown in Figure 4.8 or a half simple harmonic oscillator (SHO) potential energy well as shown in Figure 4.9 where $V(x)=+\infty$ for $x>b$. In question B-4, the energy eigenfunction for the half SHO potential energy well is oscillatory in region (II) $(a<x<b)$. The wavelength and the absolute value of the amplitude of the energy eigenfunction decrease from $x=a$ to $x=b$ since the kinetic energy of the particle increases in the semi-classical approximation. In the pre-test, only four out of seventeen students sketched the wavefunction with correct wavelength and only two students sketched the wavefunction with correct amplitude. In the post-test, ten out of twelve students were able to draw the energy eigenfunction with both correct wavelength and amplitude. Students also showed similar improvement after the QuILT when answering question A-4 about the triangular 
potential energy. The improved performance indicates that the students have a better understanding of how to qualitatively sketch the energy eigenfunction for a given quantum system in the semi-classical approach after learning the QuILT.

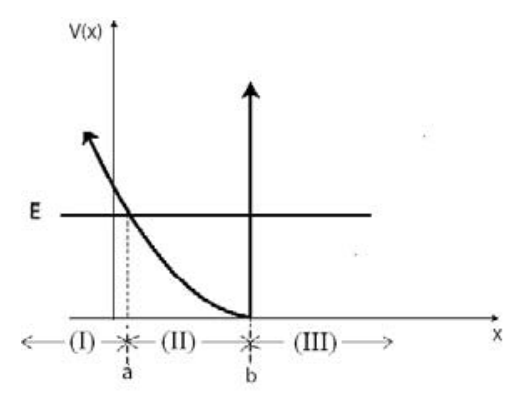

Figure 4.9 A quantum particle interacting with a half SHO potential energy well.

\subsection{CONCLUSION}

We investigated students' difficulties related to qualitatively sketching energy eigenfunctions and used the findings as a guide to develop the QuILT related to drawing energy eigenfunctions. Preliminary evaluation suggests that the QuILT about drawing energy eigenfunctions is effective in improving students' understanding of how to qualitatively sketch the energy eigenfunctions for different quantum systems in the semi-classical approximation.

\subsection{CHAPTER REFERENCE}

Chi, M. (1994). “Thinking Aloud”, M. Van Someren, Y. Barnard, and J. Sandberg (eds.), The Think Aloud Method: A Practical Guide to Modeling Cognitive Processes. Edited by London: Academic.

McKagan, S., Malley C., Adams, W., Perkins, K. and Wieman, C. (2009). Simulation program is available at $<\underline{\text { http: } / / \text { phet.colorado.edu }>}$ 


\title{
5.0 IMPROVING STUDENTS' UNDERSTANDING OF QUANTUM MEASUREMENT
}

\begin{abstract}
5.1 ABSTRACT
We describe the difficulties advanced undergraduate and graduate students have with quantum measurement and the development and implementation of research-based learning tools such as the Quantum Interactive Learning Tutorials (QuILTs) and peer instruction tools to reduce these difficulties. The preliminary evaluation shows that these learning tools are effective in improving students' understanding of concepts related to quantum measurement.
\end{abstract}

\section{$5.2 \quad$ INTRODUCTION}

In this chapter, we will discuss the investigation of students' difficulties with quantum measurement and build upon this research to develop and assess a research-based QuILT and concept tests to help students develop a good understanding of the formalism of quantum measurement. The investigation of students' difficulties with quantum measurement was conducted with the undergraduate and graduate students at the University of Pittsburgh (PITT) and other universities (Singh 2006, Singh 2007) by administering written tests and by conducting in-depth individual interviews with a subset of students. 
The research based learning tools including the QuILT and concept tests related to quantum measurement were administered to students in the first semester of a full-year juniorsenior level quantum mechanics course. They strive to build on students' prior knowledge, actively engage them in the learning process and help them build links between the abstract formalism and conceptual aspects of quantum physics without compromising the technical content. To assess the effectiveness of the QuILT and concept tests, we gave the same assessment related to quantum measurement to the experimental group and a comparison group in different but equivalent classes at two similar universities. The comparison group only had traditional lectures and weekly homework in a similar two-semester quantum mechanics class in which the same textbook was used. Our prior investigation shows that the students' performance on surveys given in the upper-level quantum mechanics courses at the two universities (experimental group and comparison group) were comparable when traditional instruction was used at both institutions.

\subsection{BACKGROUND}

Quantum measurement formalism is quite challenging. Unlike classical mechanics, where position and momentum of a particle evolve in a deterministic manner based upon the interactions, position, momentum and other observables are in general not well-defined for a given state of a quantum system. According to quantum theory, the Time dependent Schroedinger Equation (TDSE) governs the time evolution of the state which can be written as a linear superposition of a complete set of eigenstates of any hermitian operator corresponding to a

physical observable. The state of the system evolves in a deterministic manner depending on the 
Hamiltonian of the system. According to the Copenhagen interpretation, quantum measurement would instantaneously collapse the wavefunction (or the state of the system) to an eigenstate of the operator corresponding to the physical observable measured and the measured value is the corresponding eigenvalue. For example, if we measure the position of a quantum particle in a one-dimensional (1D) infinite square well, its wavefunction will collapse to a position eigenfunction which is a delta function in the position representation. If we measure its energy instead, the wavefunction of the system will collapse into an energy eigenfunction, which is a sinusoidal function inside the 1D well and goes to zero at the two boundaries.

In an $N$ dimensional Hilbert space, an operator $\hat{Q}$ corresponding to a physical observable $Q$ has $N$ eigenvalues $q_{n}$ and corresponding eigenstates $\left|q_{n}\right\rangle$. The eigenvalue spectrum of an operator can either be discrete or continuous or a combination of the two. The state of the system at a given time $t,|\Psi(t)\rangle$, can be written as a linear superposition of a complete set of eigenstates of $\left|q_{n}\right\rangle$. By projecting the wavefunction of the system $|\Psi(t)\rangle$ at time $t$ onto an eigenstate $\left|q_{n}\right\rangle$ of the operator $\hat{Q}$, we can find the probability $\left|\left\langle q_{n} \mid \Psi(t)\right\rangle\right|^{2}$ of obtaining $q_{n}$ when the observable $Q$ is measured at time $t$.

After the measurement of the observable $Q$, the time-evolution of the state of the system, which is an eigenstate of $\hat{Q}$ right after the measurement, is again governed by the time dependent Schrödinger equation (TDSE). Right after the measurement of energy, the state of the system is an energy eigenstate, and the probability density does not change with time since the only change in the wavefunction with time is an overall time-dependent phase factor. If the system is initially in an energy eigenstate at time $t=0$ and we measure an arbitrary physical observable $Q$ after a time $t$, the probability of obtaining an eigenvalue $q$ will be time- 
independent since the system was still in an energy eigenstate at time $t$ at the instant the measurement of Q was performed. Therefore, the energy eigenstates are called the stationary states. On the other hand, measurement of position would collapse the system into a position eigenstate at the instant the measurement is made. However, since the position eigenstate is a linear superposition of the energy eigenstates, the different energy eigenstates in the linear superposition will evolve with different time-dependent phase factors, and the probability density will change with time. In this case, the probability of measuring a particular value of energy will be time-independent but the probability of measuring another physical observable whose operator does not commute with the Hamiltonian will depend on time.

\subsection{INVESTIGATION OF STUDENTS' DIFFICULTIES}

Our goal was to examine students' knowledge of quantum measurement after traditional instruction. To simplify the mathematics and focus on the concepts related to quantum measurement, we often used the 1-D infinite square well model during the investigation of students' difficulties. Both open-ended questions and multiple choice questions were administered to probe students’ difficulties.

\subsubsection{Difficulty in Distinguishing between Eigenstates of Operators corresponding to Different Observables}

The measurement of a physical observable collapses the wavefunction of the quantum system into an eigenstate of the corresponding operator. Many students have difficulties distinguishing 
between energy eigenstates and the eigenstates of other physical observables. To investigate the pervasiveness of this difficulty in distinguishing between the eigenstates of different physical observables, we designed the following multiple choice question.

- Choose all of the following statements that are correct:

(1) The stationary states refer to the eigenstates of any operator corresponding to a physical observable.

(2) If a system is in an eigenstate of any operator that corresponds to a physical observable, it stays in that state unless an external perturbation is applied.

(3) If a system is in an energy eigenstate at time $t=0$, it stays in the energy eigenstate unless an external perturbation is applied.
A. 1 only
B. 3 only
C. 1 and 3 only
D. 2 and 3 only
E. all of the above

The correct answer is B (3 only). In statement (1), the stationary states should refer to the energy eigenstates only. A complete set of eigenstates of an arbitrary operator $\hat{Q}$ cannot be stationary states if $\hat{Q}$ does not commute with the Hamiltonian operator $\hat{H}$ of the system.

In the comparison group, none of the 25 students selected the correct choice. The most common incorrect choice was E (all of the above). Nearly half of the students thought that all three statements were correct because they had difficulty in differentiating between the related concepts of stationary states and eigenstates of other observables. Some students selected choice A (1 only) which is interesting because one may expect that students who believed statement (1) was correct and understood why a stationary state is called so may think that statement (2) is correct as well. In particular, for students who believed statement (1) is correct, statement (2) may be considered "a system in a stationary state stays in that state unless an external perturbation is applied", which described the property of stationary state. However, students who 
selected choice A did not relate the stationary state with the special nature of the time evolution in that state. In other words, they did not contemplate why the energy eigenstates are called “stationary states".

\subsubsection{Difficulty with possible outcomes of a measurement}

The following multiple choice question was administered to investigate students' understanding of the possible outcomes of a measurement given the state of the system for a particle in a 1D infinite square well when the measurement is performed. $\psi_{1}(x)$ and $\psi_{2}(x)$ are the ground state and first excited state wavefunctions.

- An electron is in the state given by $\frac{\psi_{1}(x)+\psi_{2}(x)}{\sqrt{2}}$. Which one of the following outcomes could you obtain if you measure the energy of the electron?
A. $E_{1}+E_{2}$
B. $\left(E_{1}+E_{2}\right) / 2$
C. Either $E_{1}$ or $E_{2}$
D. Any of the $E_{n}(n=1,2,3, \ldots)$

E. Any value between $E_{1}$ and $E_{2}$

Forty percent of the students chose the correct answer $C$ (either $E_{1}$ or $E_{2}$ ). Because the energy eigenstates $\left|\psi_{n}\right\rangle$ are orthogonal to each other, $\left|\left\langle\psi_{n} \mid \Psi\right\rangle\right|^{2}=1 / 2$ for $\mathrm{n}=1$ or $\mathrm{n}=2$ and $\left|\left\langle\psi_{n} \mid \Psi\right\rangle\right|^{2}=0$ for all the other energy eigenstates $E_{n}(n>2)$. Therefore, we can only obtain $E_{1}$ or $E_{2}$ with equal probability but no other energy. 
The most common incorrect choice, selected by $27 \%$ of the students, is $\mathrm{B}\left(\left(E_{1}+E_{2}\right) / 2\right)$. Because the probability of measuring $E_{1}$ and $E_{2}$ is $1 / 2$ each, the expectation value of energy is $\left(E_{1}+E_{2}\right) / 2$. However, these students incorrectly believe that the expectation value is the measured value of energy. The individual think-aloud interviews indicate that many students are not only confused about the distinction between individual measurements and expectation values, they also have difficulty distinguishing between the probability of measuring a particular value of an observable in a given state and the measured value or the expectation value. For example, during individual interviews, students often noted $\left\langle\psi_{n}|\hat{H}| \psi_{n}\right\rangle$ or even $\langle\Psi|\hat{H}| \Psi\rangle$ as the probability of measuring $E_{n}$ in the state $|\Psi\rangle$. When these students were explicitly asked to compare their expressions for the probability of measuring a particular value of energy and the expectation value of energy, some students appeared concerned. They realized that these two concepts were different but they generally struggled to distinguish these concepts. They could not write an expression for the probability of measuring $E_{n}$ either using the Dirac notation or in the position space representation using the integral form.

Also, some interviewed students had difficulty in connecting the probability of measuring each possible value and the expectation value of that observable in a given state. Since the expectation value in a given state equals the average of a large number of measurements of the physical observable on identically prepared systems, it is equal to the sum of the eigenvalues of the corresponding operator times their probabilities in the given state. For example, suppose we have an ensemble of $N$ identical quantum systems. If we measure the physical observable $Q$ with a discrete eigenvalue spectrum in state $|\Psi\rangle$, the estimated probability of obtaining an eigenvalue of $Q, q_{i}$, equals $n_{i} / N$ and the estimated expectation value of $Q$ in that state is 
$\sum_{i} \frac{q_{i} n_{i}}{N}$. Many students had difficulty with the statistical interpretation of the expectation value of $Q$ as the average of a large number of measurements on identically prepared systems in state $|\Psi\rangle$. A survey question was administered to 202 graduate students in seven universities as shown below (Singh 2008a):

- The wavefunction of an electron in a $1 D$ infinite square well of width a at time $t=0$ is given by $\Psi(x, 0)=\sqrt{2 / 7} \psi_{1}(x)+\sqrt{5 / 7} \psi_{2}(x)$. Answer the following questions.

(a) You measure the energy of an electron at time $t=0$. Write down the possible values of the energy and the probability of measuring each.

(b) Calculate the expectation value of the energy in the state $\Psi(x, t)$.

About $67 \%$ of the graduate students answered question (a) correctly and $7 \%$ of the graduate students confused the energy eigenvalues with the expectation value of energy. However, only 39\% of the students provided the correct response for question (b). Many students who can calculate the probabilities for each energy eigenvalue were not able to use these probabilities to find the expectation value and some of them had difficulties in realizing that the expectation value of energy is independent of time.

\subsubsection{Difficulty with the probability of measuring energy}

When we explicitly asked undergraduate students to find the probability of obtaining energy $E_{2}$ for the state $\frac{\left|\psi_{1}\right\rangle+\left|\psi_{2}\right\rangle}{\sqrt{2}}$ in a $1 \mathrm{D}$ infinite square well, many of them could provide the correct answer $1 / 2$ by observing the coefficients. To evaluate whether students could calculate the probability of measuring a particular value of energy by projecting the state vector along the 
corresponding energy eigenstate for the case where the wave function is not written explicitly in terms of a linear superposition of energy eigenstates, we designed the following question about a triangle shaped wavefunction in a $1 \mathrm{D}$ infinite square well:

- The state of an electron at $t=0$ is given by $\Psi(x)=A x$ when $0<x<\frac{a}{2}, \Psi(x)=A(a-x)$ when $\frac{a}{2} \leq x<a$ and $\Psi(x)=0$ elsewhere. Here $A$ is the normalization constant. What is the probability that an energy measurement at time $t=0$ yields $E_{2}$ ? (If there is an integral in your expression for the probability, you need not evaluate the integral but set it up properly with appropriate limits.)

Unlike the wavefunction $\frac{\left|\psi_{1}\right\rangle+\left|\psi_{2}\right\rangle}{\sqrt{2}}$ which is composed of only two energy eigenstates, the triangle function state $|\Psi\rangle$ is a superposition of infinitely many energy eigenstates, $|\Psi\rangle=\sum_{n=1}^{\infty} c_{n}\left|\psi_{n}\right\rangle$. The expansion coefficient $c_{n}$ equals $\left\langle\psi_{n} \mid \Psi\right\rangle=\int_{-\infty}^{+\infty} \psi_{n}^{*}(x) \Psi(x) d x$ and $\left|c_{n}\right|^{2}$ is the probability of obtaining $E_{n}$ when energy is measured for state $|\Psi\rangle$. Thus, to answer this question correctly, students need to write $|\Psi\rangle$ as a linear superposition of $\left\{\left|\psi_{n}\right\rangle\right\}$ and find the component of $|\Psi\rangle$ along $\left|\psi_{n}\right\rangle$.

Only one student out of fifteen provided the correct answer for the probability of measuring $E_{n}$. Some students left this question blank. Other students made two typical common mistakes. Twenty percent of the students wrote down the energy expectation value $\langle\Psi|\hat{H}| \Psi\rangle$ to represent the energy measurement probability. In further interviews with some students, we asked how the expression $\langle\Psi|\hat{H}| \Psi\rangle$ which only involves the state $|\Psi\rangle$ will favor energy $E_{2}$ over 
any other energy. Some of the students then changed their answers to $\left\langle\psi_{2}|\hat{H}| \Psi\right\rangle$ which was still incorrect. Another $27 \%$ of the students believed that the "probability" of measuring any physical observable was represented by $|\Psi(x)|^{2}$ according to the interpretation of wavefunction. These students were confusing the probability density of measuring position with the probability of measuring other physical observables such as energy.

A similar multiple-choice question about a parabola shaped wavefunction was administered to 76 undergraduate students in six universities as shown below:

- Consider the following wavefunction for a $1 D$ infinite square well: $\psi(x)=A x(a-x)$ for $0 \leq x \leq a$ and $\psi(x)=0$ otherwise. $A$ is a normalization constant. Which one of the following expressions correctly represents the probability of measuring the energy $E_{n}$ for the state $\psi(x)$ ?
A. $\left|\int_{0}^{a} \psi_{n}^{*}(x) \hat{H} \psi(x) d x\right|^{2}$
B. $\left|\int_{0}^{a} \psi_{n}^{*}(x) \psi(x) d x\right|^{2}$
C. $\left|\psi_{n}^{*}(x) \hat{H} \psi(x)\right|^{2}$
D. $\left|\psi_{n}^{*}(x) \psi(x)\right|^{2}$
E. $|\psi(x)|^{2}$

Only 33\% of the students chose the correct answer B. $45 \%$ of the students incorrectly selected the distractor A which is an equivalent expression for $\left|\left\langle\psi_{2}|\hat{H}| \Psi\right\rangle\right|^{2}$. Another multiple choice question given to the same 76 students asked about the energy measurement outcome for the state $\sqrt{4 / 7}\left|\psi_{1}\right\rangle+\sqrt{3 / 7}\left|\psi_{2}\right\rangle .55 \%$ of the students provided the correct answer. $21 \%$ of the students incorrectly thought that other energies $E_{n}$ besides $E_{1}$ and $E_{2}$ could also be obtained while the probability of measuring $E_{1}$ would be largest. Another $12 \%$ of the students thought all the possible energies $E_{n}$ can be measured with the same probability. 


\subsubsection{Measurement and future time evolution of the wavefunction}

Within the Copenhagen interpretation of quantum mechanics, the measurement of an observable is treated separately from the "normal" time-evolution of the system according to the TDSE. When a measurement is performed, the state of the system instantaneously collapses to an eigenstate of the operator corresponding to the observable measured after which the system will evolve normally according to the TDSE. We investigated students' understanding of the timedevelopment of the wavefunction according to the TDSE after the measurement of an observable and its effect on consecutive measurements by asking students the following consecutive position measurements question about a 1D infinite square well:

- If you make a measurement of position on an electron in the ground state and wait for a long time before making a second measurement of position, do you expect the outcome to be the same in the two measurements? Explain.

To correctly answer this question, students must know the following: (1) The ground state wavefunction will collapse into a position eigenfunction (a delta function) after the first position measurement. (2) The position eigenfunction is not a stationary state wavefunction so the wavefunction will evolve in time in a non-trivial manner and it will not in general be found in a position eigenstate at a time $t$. Therefore, after a long time, the second measurement of position in general will yield a different value from the first measurement. We note however that in an infinite square well, the time evolution of the system is such that the wave function repeats itself with a certain periodicity (A detailed discussion of this periodicity in time for an infinite square well is in the appendix of this chapter, i.e., section 5.10.). 
Difficulity 1: System remains in the energy eigenstate after a position measurement.

In response to this question, some students thought that the system will be in the ground state after both the first and second position measurements. Interviews suggest that students with these types of responses often did not realize the difference between an energy eigenstate and position eigenstate. In the written survey, only one student explicitly mentioned the wavefunction collapse after the first position measurement. However, his response was “...the wavefunction collapses into the measured state" and he did not elaborate that the "measured state” is actually a position eigenstate.

Difficulty 2: System stays in the position eigenstate at any time after a position measurement.

On the other hand, some students believed that after the first position measurement the system gets "stuck" in a position eigenstate and did not know that the position eigenfunction evolves in time in a non-trivial manner and the system does not remain a position eigenfunction for all future time $t$. These students believed that the second position measurement even after a long time will give the same value as the first one unless there was an "outside disturbance" between the two measurements. Only two students out of fifteen mentioned the correct time evolution of the quantum mechanical system after the position measurement.

Difficulty 3: System finally goes back to the initial state

Students were also asked another series of measurement related questions when the initial state of the system is $\psi(x, 0)=\sqrt{2 / 7} \psi_{1}(x)+\sqrt{5 / 7} \psi_{2}(x)$ for an electron confined in a 1D infinite square well as follows: 
- Q1. If the energy measurement yields $4 \pi^{2} \hbar^{2} /\left(2 m a^{2}\right)$, what is the wavefunction right after the measurement?

- Q2. Immediately after the energy measurement in Q1, you measure the position of the electron. What possible values could you obtain and what is the probability of each?

- Q3. After the position measurement in Q2, you wait for time $t$ and measure the position again. Would the probability of measuring each possible value be different from your answer to Q2?

Q1 has been given as a multiple-choice question to 89 students in 6 universities asking about the state of the system long after the energy measurement. $20 \%$ of the students did not know the wavefunction would collapse at the instant the energy was measured. $36 \%$ of the students thought the wavefunction will collapse upon the energy measurement but eventually evolve back to the initial state $\sqrt{2 / 7} \psi_{1}(x)+\sqrt{5 / 7} \psi_{2}(x)$ a long time after the measurement. During the individual interview, a student said. “...it’s like tossing a coin. You can get either head or tail after the measurement. But when you make another measurement, it goes back to a coin (with two sides)."

Difficulty 4. Probability density for position measurement

Born's probabilistic interpretation of the wavefunction can also be confusing for students. In Q2, we hoped that students would note that one can measure position values between $x=0$ and $x=a$ (except at $x=0, a / 2, a$ where the wavefunction is zero), and according to Born's interpretation, $|\psi|^{2} \mathrm{~d} x$ gives the probability of finding the particle in a narrow range between $x$ and $x+\mathrm{d} x$. Only $38 \%$ of the students provided the correct response. Partial responses were considered correct for tallying purposes if students wrote anything that was correct related to the 
above wavefunction, e.g., "The probability of finding the electron is highest at $a / 4$ and $3 a / 4 . "$, “The probability of finding the electron is non-zero only in the well”, etc.

Eleven percent of the students tried to find the expectation value of position instead of the probability of finding the electron at a given position. They wrote the expectation value of position in terms of an integral involving the wavefunction. Many of them explicitly wrote that probability $=(2 / a) \int_{0}^{a} x \sin ^{2}(2 \pi x / a) d x$ and believed that instead of the expectation value they were calculating the probability of measuring the position of the electron.

During the interview, one student said (and wrote on paper) that the probability is $\int x|\psi|^{2} d x$. When the interviewer asked why $|\psi|^{2}$ should be multiplied with $x$ and if there is any significance to $|\psi|^{2} d x$ alone without multiplying it by $x$, the student said, “ $|\psi|^{2}$ gives the probability of the wavefunction being at a given position and if you multiply it by $x$ you get the probability of measuring (student's emphasis) the position $x$ ". When the student was asked questions about the meaning of the "wavefunction being at a given position", and the purpose of the integral and its limits, the student was unsure. He said that the reason he wrote the integral is because $x|\psi|^{2} d x$ without an integral looked strange to him. Similar confusion about probability in classical physics situations has been found.

Difficulty 5. Use classical experience to analyze time evolution in quantum systems

No students could answer Q3 correctly though it assesses the same concepts as in the consecutive position measurement question discussed earlier. In the consecutive position measurement question, some students used a classical description to answer this question about the time-evolution after the measurement such as "the electron moves around". Such classical 
responses reflect students' discomfort describing the time evolution of a quantum system in terms of the time-development of the wavefunction. Therefore, they could not answer Q3 since no classical element such as "particle” was involved.

\subsubsection{An operator acting on a state corresponds to a measurement of the corresponding observable}

One of the questions on a survey given to more than 200 graduate students asked them to consider the following statement: "By definition, the Hamiltonian acting on any allowed (possible) state of the system $|\psi\rangle$ will give the same state back, i.e., $\hat{H}|\psi\rangle=E|\psi\rangle$, where $E$ is the energy of the system.” Students were asked to explain why they agree or disagree with this statement. We wanted students to disagree with the statement and note that it is only true if $|\psi\rangle$ is a stationary state. In general, $|\psi\rangle=\sum_{n=1}^{\infty} C_{n}\left|\psi_{n}\right\rangle$, where $\left|\psi_{n}\right\rangle$ are the stationary states and $C_{n}=\left\langle\psi_{n} \mid \psi\right\rangle$. Then, $\hat{H}|\psi\rangle=\sum_{n-1}^{\infty} C_{n} E_{n}\left|\psi_{n}\right\rangle \neq E|\psi\rangle$.

Eleven percent of the students answering this question incorrectly believed that any statement involving a Hamiltonian operator acting on a state is a statement about the measurement of energy.

Some of these students who incorrectly claimed that $\hat{H}|\psi\rangle=E|\psi\rangle$ is a statement about energy measurement agreed with the statement while others disagreed. Those who disagreed often claimed that $\hat{H}|\psi\rangle=E_{n}\left|\psi_{n}\right\rangle$ because as soon as $\hat{H}$ acts on $|\psi\rangle$, the wavefunction will 
collapse into one of the stationary states $\left|\psi_{n}\right\rangle$ and the corresponding energy $E_{n}$ will be measured. The following are two typical responses in this category:

* Disagree. Hamiltonian acting on a state (measurement of energy) will return an energy eigenstate.

* When $|\psi\rangle$ is a superposition state and $\hat{H}$ acts on $|\psi\rangle$, then $|\psi\rangle$ evolves to one of the $\left|\psi_{n}\right\rangle$ so we have $\hat{H}|\psi\rangle=E_{n}\left|\psi_{n}\right\rangle$.

Interviews and written reasonings suggest that these students believed that the measurement of any physical observable in a particular state is achieved by acting with the corresponding operator on the state. The incorrect notions expressed above are often overgeneralizations of the fact that after the measurement of energy, the system is in a stationary state so $\hat{H}\left|\psi_{n}\right\rangle=E_{n}\left|\psi_{n}\right\rangle$.

Individual interviews related to this question suggest that some students believed that whenever an operator $\hat{Q}$ corresponding to a physical observable $\mathrm{Q}$ acts on any state $|\psi\rangle$, it will yield a corresponding eigenvalue $\lambda$ and the same state back, i.e., $\hat{Q}|\psi\rangle=\lambda|\psi\rangle$ or would yield $\hat{Q}|\psi\rangle=\lambda_{n}\left|\phi_{n}\right\rangle$ (but $\hat{Q}\left|\phi_{n}\right\rangle=\lambda_{n}\left|\phi_{n}\right\rangle$ in reality).

We further explored this issue by asking 17 and 15 graduate students at the end of their first semester and second semester graduate level quantum mechanics course the following question. The 15 graduate students were the same both semesters.

- $\quad$ Consider the following conversation between Andy and Caroline about the measurement of an observable $Q$ for a system in a state $|\psi\rangle$ which is not an eigenstate of $\hat{Q}$ : 
Andy: When an operator $\hat{Q}$ corresponding to a physical observable $Q$ acts on the state $|\psi\rangle$, it corresponds to a measurement of that observable. Therefore, $\hat{Q}|\psi\rangle=q|\psi\rangle$ where $q$ is the observed value.

Caroline: No. The measurement collapses the state so $\hat{Q}|\psi\rangle=q\left|\psi_{q}\right\rangle$ where $\left|\psi_{q}\right\rangle$ on the right hand side of the equation is an eigenstate of $\hat{Q}$ with eigenvalue $q$. With whom do you agree?
A. Agree with Caroline only
B. Agree with Andy only
C. Agree with neither
D. Agree with both
E. The answer depends on the observable $Q$.

We note that the question was not posed as a multiple-choice question at the end of the first semester course but students were asked to explain with whom if any they agreed and why. There was a brief discussion of the correct response to the question after administering the survey in which this question was asked. At the end of the first semester course, $12 \%$ of the students agreed with Andy, 47\% with Caroline, 29\% with neither (correct response) and 12\% provided no response. At the end of the second semester course, 13\% of the students agreed with Andy, 20\% with Caroline, 7\% with both and 53\% with neither (correct response). While the percentage of correct response increased significantly from the first to the second administration, many students still had difficulty with this concept. Earlier, the version of this question not in the multiple-choice format was posed to 37 graduate students at the beginning of their graduate level quantum mechanics course (not the same students as those who answered it at the end of the first and second semester of their graduate level quantum mechanics course). In that group, $24 \%$ of the students agreed with Andy, 54\% with Caroline and 22\% with neither (correct response). Indeed this difficulty is quite common even amongst graduate students. 
In summary, students have difficulty in differentiating between the stationary states and the eigenstates of an arbitrary operator. They have difficulty in determining the possible values one may obtain upon measurement of an observable in a given state and the possible states the system could collapse into upon a measurement. Moreover, they had difficulty in calculating the probability of obtaining a particular eigenvalue when an observable is measured in a given state. Students often were confused between outcomes of individual measurements, expectation value and probability of measuring a particular value of the observable. Many students believed that an operator acting on a state corresponds to the measurement of that observable. They also had difficulty in analyzing the time development of the eigenstates of operators corresponding to different observables after a quantum measurement. They often incorrectly believe that after a measurement, the state of the system would either be stuck in the eigenstate in which it collapsed or go back to the initial state before the measurement was performed (and remain there).

\subsection{WARM-UP FOR THE QUILT ON QUANTUM MEASUREMENT}

The measurement QuILT begins with warm-up exercises that students work on before working on the QuILT. We designed the warm-up to help students review the concept of eigenstate and to help them understand that the eigenstates of all physical observables are not the same. First, we let students consider the difference between the energy eigenstates and a possible wavefunction which was a linear superposition of the energy eigenstates. Questions were also designed to help students understand that an energy eigenstate satisfies the time independent Schrödinger equation (TISE) but its linear superposition with different energies does not satisfy the TISE. In addition to the questions in verbal and mathematical representations that asked students to 
consider the differences between the energy eigenstates and their linear superpositions, one question asked them to select the energy eigenstates from three pictorial representations as shown in Figure 5.1 (in which the first two were sinusoidal) for a 1D infinite square well.

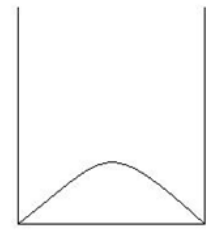

( I)

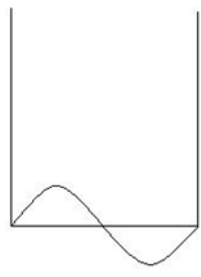

( II )

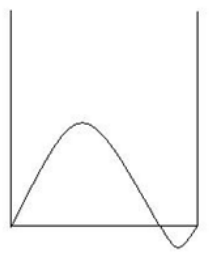

( III )

Figure 5.1 Pictorial question in the warm-up testing about the energy eigenstates for a $1 \mathrm{D}$ infinite square well. (I) and (II) are energy eigenstates but their superposition (III) is not.

Pictures (I) and (II) in Fig 1 correspond to the ground and first excited state wavefunctions $\psi_{1}$ and $\psi_{2}$ respectively. Picture (III) is one particular linear superposition of (I) and (II) $\left(\psi_{1}+\psi_{2}\right)$. The students learn that the energy eigenstates for this system are even or odd about the center of the well but their superpositions need not be. After the 1D infinite square well model, similar considerations were reinforced using the simple harmonic oscillator (SHO) model. From these two models, students learned that the eigenfunctions of different Hamiltonians have different shapes but they satisfy the TISE for the respective systems because they are states with definite energy. Students were required to summarize these characteristics of the energy eigenstates after they studied these two examples in the warm-up.

The position eigenstate was also important in helping students understand the concept of an eigenstate and the fact that not all eigenstates are energy eigenstates. Students were asked to draw a position eigenfunction with an eigenvalue $x_{0}$ for a particle interacting with an infinite square well and a finite square well. Students learned that unlike the energy eigenfunctions, the position eigenfunctions have the same shape for all the $1 \mathrm{D}$ systems and their shape has nothing 
to do with the Hamiltonian of the system. Students learned about the mathematical representation of a position eigenfunction as a delta function and the eigenvalue equation for the position operator $\hat{x} \psi\left(x_{0}\right)=x_{0} \psi\left(x_{0}\right)$. Students were explicitly asked to compare the position eigenfunction and the energy eigenfunction. In one question, they were asked to consider the following statement and explain why they agreed or disagreed:

"The position eigenstate and energy eigenstate are the same for a given system. After all, they are all eigenstates." Explain why you agree or disagree with this statement."

The warm-up helped students learn about the properties of eigenstates of the operators corresponding to different physical observables. Students learned that eigenstates of different operators are different and that they satisfy an eigenvalue equation for that operator. They also learned that if the system is in an eigenstate of an operator corresponding to a physical observable, that observable is well-defined in that state and its measurement will yield a definite value with $100 \%$ probability.

\subsection{QUILT FOR QUANTUM MEASURMENT PART I}

The goal of the measurement QuILT is to build connections between the formalism and conceptual aspects of quantum measurement without compromising the technical aspects (Singh 2008b). The measurement QuILT can be implemented in class so that two or three students work together on it or it can also be given to the students as homework or self-learning materials. The main measurement QuILT was divided into two parts: the first part dealt with outcomes of measurement and the probability of obtaining those outcomes whereas the second part dealt with time-evolution after the measurement. 
The measurement QuILT builds on the prior knowledge of students and was developed taking into account the difficulties found in the written surveys and interviews. QuILT development went through a cyclical iterative process which includes the following stages: (1) Development of the preliminary version based upon theoretical analysis of the underlying knowledge structure and research on students' difficulties, (2) Implementation and evaluation of the QuILT by administering it individually to students, measuring its impact on student learning and assessing what difficulties remained, (3) refinement and modification based upon the feedback from the implementation and evaluation.

The individual interviews were carried out using a think-aloud protocol to better understand the rationale for their responses before, during and after the development of different versions of the measurement QuILT and the corresponding pre-test and post-test. During the semi-structured interviews, students were asked to verbalize their thought processes while they answered questions about measurement either as separate questions before the preliminary version of the QuILT was developed or as a part of the QuILT. Students were not interrupted unless they remained quiet for a while. In the end, we asked them for clarification of the issues they had not made clear earlier. Some of these interviews involved asking students to predict what should happen in a particular situation, having them observe what happens in a simulation, and asking them to reconcile the differences between their prediction and observation. After each individual interview with a particular version of the measurement QuILT (along with the pre-test and post-test administered), modifications were made based upon the feedback obtained from students' performance on the QuILT (if students got stuck at a particular point and could not make progress from one question to the next with the hints already provided, suitable modifications were made), the pre-test and the post-test. When we found that the measurement 
QuILT was working well in individual administration and the post-test performance was significantly improved compared to the pre-test performance, it was administered in the quantum mechanics class.

The measurement QuILT uses computer-based visualization tools to help students build a physical intuition about concepts related to quantum measurement. The Open Source Physics program was adapted as needed throughout the measurement QuILT. This program is flexible and can be easily tailored to the desired situations.

One effective strategy to help students build a robust knowledge structure is by causing a state of "disequilibrium" in students' minds such that the students themselves realize that there is some inconsistency in their reasoning and then providing them appropriate guidance and support. In the measurement QuILT, after predicting what they expect in various situations, students are asked to check their predictions using simulations. If the prediction and observations do not match, students reach a state of disequilibrium. At that point the QuILT provides them guidance to help build a good grasp of relevant concepts and reconcile the differences between their predictions and observations.

\subsubsection{Outcome of Quantum Measurement}

The first model in the QuILT is the 1D infinite square well. For different initial states $\left|\psi_{1}\right\rangle$,

$\frac{1}{\sqrt{2}}\left(\left|\psi_{1}\right\rangle+\left|\psi_{2}\right\rangle\right)$ and $|\Psi\rangle=\sum A_{n}\left|\psi_{n}\right\rangle$, students predict what value they would obtain and what state the system would be in after the energy measurement. After their prediction, they run a computer simulation to examine their responses and reconcile the differences between their predictions and observations. The simulation is adapted from the open source physics 
simulations (Belloni and Christian 2003, Belloni et al. 2006). If a student's prediction is inconsistent with what he/she observes in the simulation, there is a cognitive conflict and the student may be motivated to resolve the inconsistency. Then the QuILT provides guidance to students to help them build a knowledge structure about quantum measurement.

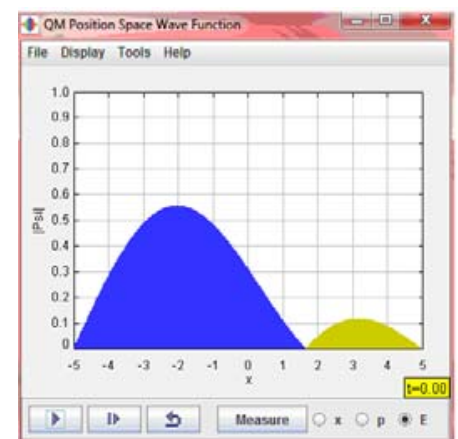

(a)

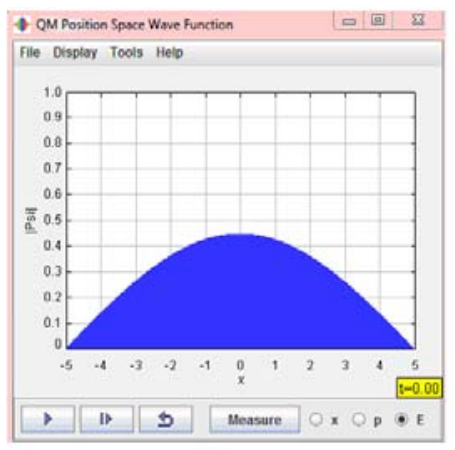

(b)

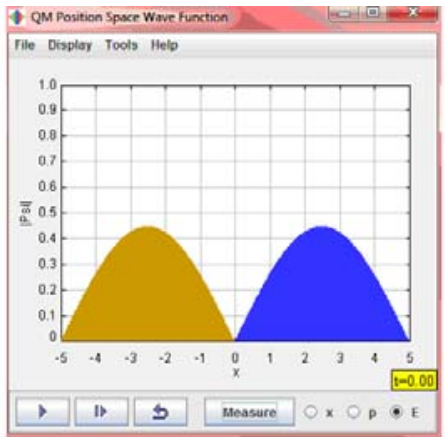

(c)

Figure 5.2 Simulation program of the energy measurement on a superposition state with two energy eigenstate components. (a) is the superposition state, (b) is the ground state and (c) is the first excited state. The phase of the wavefunction is represented by different colors.

In the simulation, one example of an initial state is $\frac{1}{\sqrt{2}}\left(\left|\psi_{1}\right\rangle+\left|\psi_{2}\right\rangle\right)$. The vertical axis of the plot in the simulation is the absolute value of the wavefunction $|\Psi|$ and the horizontal axis is the position $x$. In the simulation, students can measure position, momentum or energy. In Figure 5.2(a), students can observe the shape of $|\Psi|$ for the superposition state $\frac{1}{\sqrt{2}}\left(\left|\psi_{1}\right\rangle+\left|\psi_{2}\right\rangle\right)$ at time $t=0$. When the students measure the energy of the system, the wavefunction may collapse to $\left|\psi_{1}\right\rangle$ or $\left|\psi_{2}\right\rangle$ which are shown in Figure 5.2(b) and (c) respectively. Students observe and justify why the shape of $|\Psi|$ changed upon measurement to that of a stationary state and it does not change with time after that. 
The students are also asked to reset the initial state and repeat the measurement process several times to check whether the measurement yields the same result (the probability is $50 \%$ for obtaining $\left|\psi_{1}\right\rangle$ or $\left.\left|\psi_{2}\right\rangle\right)$. Since the state is a superposition of only two stationary states, it is possible for the students to obtain the same state for the repeated energy measurements of the initial state. Therefore, the QuILT asked students what could happen if they measured energy in the state $\sum A_{n}\left|\psi_{n}\right\rangle$ as shown in Fig 5.3, which is a linear superposition of nine stationary states $\left|\psi_{1}\right\rangle$ to $\left|\psi_{9}\right\rangle$ with equal probability. After predicting the probability of obtaining different values of energy, students were asked to measure the energy, reset $|\Psi|$ to the initial state and measure it again. Since the probability of measuring the same energy is small (but non-zero) for this superposition, students appreciated this example while working on the QuILT. To ensure that the students understood the issues related to the energy measurement in multiple contexts, the QuILT also incorporated questions for the SHO Hamiltonian.
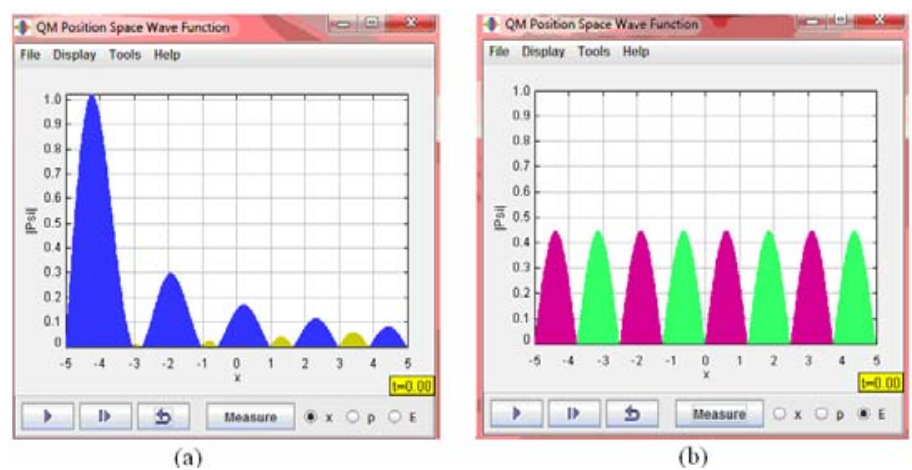

(b)

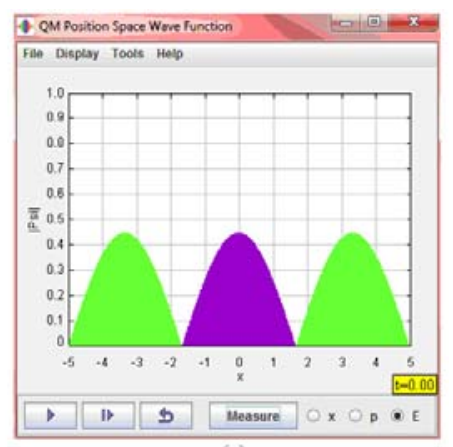

(c)

Figure 5.3 Simulation program of the energy measurement on a superposition state with nine energy eigenstate components.

The measurement QuILT also helps students with issues related to the position measurement with initial states similar to those for the energy measurement, e.g., 1D infinite square well and SHO with the initial states $\left|\psi_{1}\right\rangle$ or $\frac{1}{\sqrt{2}}\left(\left|\psi_{1}\right\rangle+\left|\psi_{2}\right\rangle\right)$. Students first predict 
theoretically what state they would obtain after a position measurement and then they use the simulation to check their prediction. In an ideal position measurement, the state of the system would collapse to a delta function at a position where the probability of measuring the position is non-zero. As shown in Figure 5.4, the initial state $\left|\psi_{1}\right\rangle$ collapses to a broad peaked Gaussian packet because of the computational limitations in constructing a very peaked function. However, the QuILT uses this opportunity to help students recognize that a delta function is a theoretical construction and the position measurement in real world situations, e.g., single particles in double slit experiment landing on the screen, would have an uncertainty in position measured.

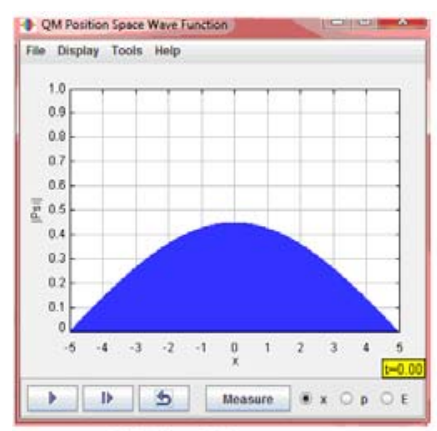

(a) Before Measurment

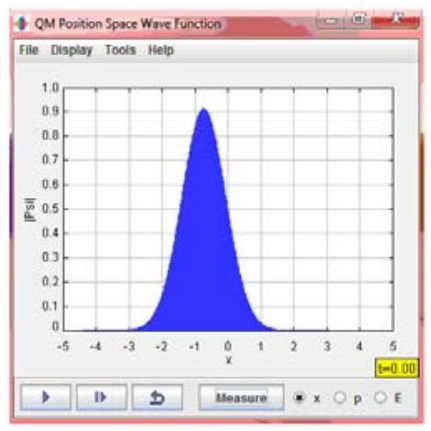

(b) After Measurement

Figure 5.4 Simulation program of the position measurement on an energy eigenstate

After asking students to predict what should happen if we perform position measurements on a large number of identically prepared systems, students are asked to reset the initial state of the system and repeat the position measurement. They observe that the center of the collapsed wavefunction is generally different but its shape is always the same. This notion is verified by the students in multiple contexts, e.g., for different quantum systems and different initial states. Students are explicitly asked to compare and contrast what they learned from the measurements of position and energy to help them understand better the outcomes of measurement for different physical observables. 


\subsubsection{Calculating the Probability of Measuring Different Values}

In addition to helping students learn about the possible outcomes of a measurement, the QuILT also teaches students how to calculate the probability of obtaining each outcomes. In surveys and individual interviews, we found that most students could find the probability of measuring different energies by observing the coefficients in an explicit superposition of stationary states,

e.g., $\frac{1}{\sqrt{2}}\left(\left|\psi_{1}\right\rangle+\left|\psi_{2}\right\rangle\right)$. So the QuILT first helps students use the projection of the initial state along an eigenstate of the operator corresponding to the observable measured to interpret these coefficients and how they may be calculated for cases for which the wavefunction may not be explicitly written as a linear superposition of stationary states. The QuILT also helps students make connection between the Dirac notation form and integral form of the inner product $\left\langle\psi_{n} \mid \Psi\right\rangle=\int \psi_{n}^{*}(x) \Psi(x) d x$ (the most common difficulty with the position representation is that students do not realize that there is an integral involved in $\left\langle\psi_{n} \mid \Psi\right\rangle$ ). Students are asked to infer the dimension (unit) of the inner product $\left\langle\psi_{n} \mid \Psi\right\rangle$ and the physical meaning of $\left|\left\langle\psi_{n} \mid \Psi\right\rangle\right|^{2}$. Students calculate these abstract inner products in concrete contexts, e.g., $|\Psi\rangle=\frac{1}{\sqrt{2}}\left(\left|\psi_{1}\right\rangle+\left|\psi_{2}\right\rangle\right)$. They learn that for this concrete case, for $n \geq 3$, the probability of obtaining energy $E_{n}$ is zero because the projection of the state $|\Psi\rangle$ along the eigenstate $\left|\psi_{n}\right\rangle$ is zero, i.e., $\left\langle\psi_{n} \mid \Psi\right\rangle=0$. After making sense of the probability for measuring energy $E_{n}$ for state $|\Psi\rangle=\frac{1}{\sqrt{2}}\left(\left|\psi_{1}\right\rangle+\left|\psi_{2}\right\rangle\right)$, students calculate the probabilities of measuring different energies for a general state $|\Psi\rangle=\sum_{n} A_{n}\left|\psi_{n}\right\rangle$ which is explicitly written as a linear superposition of stationary states. 
Students calculate that $A_{n}=\left\langle\psi_{n} \mid \Psi\right\rangle$ is the probability amplitude and $\left|\left\langle\psi_{n} \mid \Psi\right\rangle\right|^{2}$ is the probability of measuring energy $E_{n}$.

Students then learn that any possible state $|\Psi\rangle$ which is not explicitly written as a linear superposition of a complete set of eigenstates of an operator corresponding to a physical observable, e.g., energy, could be written that way. Then, the students are asked to find the coefficients in the expansion of $|\Psi\rangle$ as a linear superposition of the energy eigenstates $\left|\psi_{n}\right\rangle$ as in this example:

- The orthonormal energy eigenstates $\psi_{n}$ for a $1 D$ infinite square well satisfy $\int_{0}^{a} \psi_{n}^{*}(x) \psi_{m}(x) d x=\delta_{m n}$, where $\delta_{m n}=1$ when $m=n$, and $\delta_{m n}=0$ otherwise. Any state $|\Psi\rangle$ can be expressed as $|\Psi\rangle=\sum_{n} A_{n}\left|\psi_{n}\right\rangle$ because $\left|\psi_{n}\right\rangle$ form a complete set of vectors for the Hilbert space in which the state of the system lies. Find $A_{n}$ in terms of $|\Psi\rangle$ and $\left|\psi_{n}\right\rangle$ first in Dirac notation form and then in the integral form in the position representation .

If the students did not have the mathematical skills to answer the question above, hints were provided, e.g., about how to use the Fourier trick and multiply both sides of the expression $\Psi(x)=\sum A_{n} \psi_{n}(x)$ by $\psi_{m}^{*}(x)$ and integrate over all space. Then students calculated the probability of obtaining $E_{n}$ for a concrete example of a parabola shaped wavefunction for a 1D infinite square well for which the wavefunction was not explicitly written in terms of a linear superposition of energy eigenfunctions. Students further contemplated these issues using different states for a SHO model to learn about the projection of the state along an eigenstate of an operator corresponding to a physical observable in multiple contexts. 
The QuILT helps students learn that the probability of measuring other physical observables can be obtained by projecting the state of the system along an eigenstate of an operator corresponding to a physical observable. They use this projection method to analyze the probability density for position measurement. Earlier in the QuILT, students had already learned that $\left\langle\psi_{n} \mid \Psi\right\rangle=\int \psi_{n}^{*}(x) \Psi(x) d x$. Students were also asked to differentiate between an energy eigenfunction $\psi_{1}(x)=\sqrt{\frac{2}{a}} \sin \left(\frac{\pi x}{a}\right)$ of a $1 \mathrm{D}$ infinite square well and a position eigenfunction $\psi(x)=\delta\left(x-x_{0}\right)$ with eigenvalue $x_{0}$. They were also explicitly asked to project the ground state of the system $\left|\psi_{1}\right\rangle$ onto the position eigenstate $\left|x_{0}\right\rangle$ with eigenvalue $x_{0}$ and interpret their result. The probability density for finding the particle at the position $x_{0}$ is

$$
\left|\left\langle x_{0} \mid \psi_{1}\right\rangle\right|^{2}=\left|\int_{-\infty}^{+\infty} \delta\left(x-x_{0}\right) \sqrt{\frac{2}{a}} \sin \left(\frac{\pi x}{a}\right) d x\right|^{2}=\left|\sqrt{\frac{2}{a}} \sin \left(\frac{\pi x_{0}}{a}\right)\right|^{2} .
$$

Moreover, by the definition of wavefunction, $\left|\psi_{1}(x)\right|^{2}=\left|\left\langle x \mid \psi_{1}\right\rangle\right|^{2}$ is the probability density for finding the particle at position $x$. The QuILT required students to assimilate the Born interpretation for finding the probability density for finding the particle with the method of projecting the state vector along a position eigenstate.

After students had learned about the probability density for position measurement using the projection method, the QuILT explicitly brought up a common difficulty they have in differentiating between the probability of obtaining a particular value, the expectation value and similar looking expressions. For example, students were asked to consider the following statement: 
- If the initial state is $|\Psi\rangle$ for a particle in a 1-D infinite square well, $\left|\left\langle\psi_{1}|H| \Psi\right\rangle\right|^{2}$ is the probability of obtaining energy $E_{1}$ when measuring the energy of the particle. Do you agree with this statement? Explain.

Students were given hints to consider the dimension (units) of $\left\langle\psi_{1}|H| \Psi\right\rangle$. They were also asked to consider the physical meaning of $\langle\Psi|H| \Psi\rangle$ and $\langle\Psi|x| \Psi\rangle$ (in terms of the average of a large number of measurements on identically prepared systems). In the warm-up, students had learned that the energy eigenstates $\left|\psi_{n}\right\rangle$ satisfy the TISE $\hat{H}\left|\psi_{n}\right\rangle=E_{n}\left|\psi_{n}\right\rangle$. By decomposing the general state $|\Psi\rangle$ into a linear superposition of $\left|\psi_{n}\right\rangle$, they learn that $\left\langle\psi_{1}|H| \Psi\right\rangle=E_{1}\left\langle\psi_{1} \mid \Psi\right\rangle$ which has the dimension of energy. They also contemplate the fact that $\langle\Psi|H| \Psi\rangle=\sum_{n}\left|A_{n}\right|^{2} E_{n}$, the expectation value of the energy in state $|\Psi\rangle$, is the average of a large number of measurements on identically prepared systems. In an analogous manner, they interpret the expectation value of position $\langle\Psi|x| \Psi\rangle$. Explicit comparison of the expectation values with the measurement probabilities is designed to help students distinguish between these related concepts.

\subsection{QUILT FOR QUANTUM MEASURMENT PART II}

The second part of the measurement QuILT focuses on the time development of a quantum system after a measurement. After an energy measurement, the system would collapse into a stationary state and remain in that state till another measurement is performed. However, if we measure, e.g., the position of the particle, the wavefunction of the system will change with time. 
In the QuILT, the system behaviors after the energy measurement and the position measurement were explicitly compared to help students learn about the differences in the time evolution in a stationary and a non-stationary state.

\subsubsection{Energy Measurement}

In the first part of the measurement QuILT, students learn about the possible outcomes of the energy measurement in a 1D infinite square well for three different cases where the states of the system are $\left|\psi_{1}\right\rangle, \frac{1}{\sqrt{2}}\left(\left|\psi_{1}\right\rangle+\left|\psi_{2}\right\rangle\right)$ and $\sum A_{n}\left|\psi_{n}\right\rangle$ at time $t=0$ when the measurements are performed. At the beginning of the second part of the measurement QuILT, we ask students about the possible values of the energy measurement if we started with the same three initial states but performed the measurement at a time $t>0$. Also, they are explicitly asked to write the states of the system right before the measurement in each case. For example, if the initial state is $\left|\psi_{1}\right\rangle$, the wavefunction at time $t$ would be $\left|\psi_{1}\right\rangle e^{-i E_{1} t / \hbar}$, which is still the ground state, and the energy measurement will yield the ground state energy $E_{1}$ with $100 \%$ probability. If the initial state is $\frac{1}{\sqrt{2}}\left(\left|\psi_{1}\right\rangle+\left|\psi_{2}\right\rangle\right)$, the state of the system would evolve into $\frac{1}{\sqrt{2}}\left(\left|\psi_{1}\right\rangle e^{-i E_{1} t / \hbar}+\left|\psi_{2}\right\rangle e^{-i E_{2} t / \hbar}\right)$ after a time $t$. Thus, the probability of measuring energy is unchanged (in this case $50 \%$ each for the ground and first excited state energies) even if the system is in a linear superposition of stationary states. Many students correctly predicted that the energy measurement at time $t>0$ would yield the same values $E_{1}$ and $E_{2}$ as at time $t=0$ but they incorrectly justified it by saying that the wavefunction after a time $t$ is the same as that at time $t=0$. Students were asked to check their prediction with a simulation showing the time evolution of the absolute value of the 
wavefunction with two energy eigenstate components. After they observed that the shape of the wavefunction changes with time as shown in Figure 5.5, contrary to their initial prediction, they were in a state of disequilibrium.
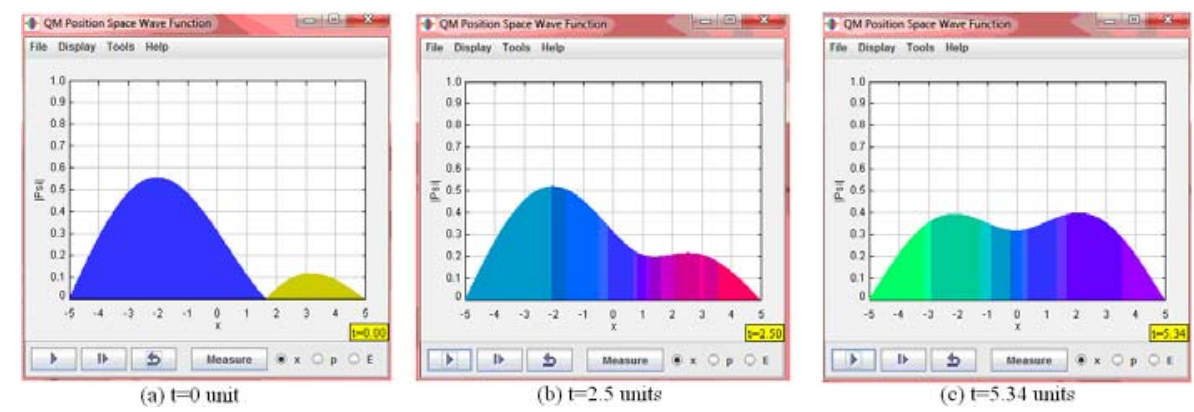

Figure 5.5 Time evolution on a superposition state with two energy eigenstate components. (a) is the absolute value of the initial state wavefunction and (b) and (c) are the absolute values of the wavefunction at different times. The phases of the wavefunction are represented by different colors.

They were also asked to measure the energy several times at different times, e.g., $t=2$ or 3 units after resetting the system to the same initial state after each measurement. They realized that the system only collapsed into $\left|\psi_{1}\right\rangle$ or $\left|\psi_{2}\right\rangle$. At this point, the QuILT helped them reason systematically about why the probability of measuring different values of energies does not change with time even though the shape of the wavefunction changes with time for the state $|\Psi\rangle=\frac{1}{\sqrt{2}}\left(\left|\psi_{1}\right\rangle e^{-i E_{1} t / \hbar}+\left|\psi_{2}\right\rangle e^{-i E_{2} t / \hbar}\right)$.

Some students held the misconception that the state of the system after the measurement would eventually go back to the initial state before the measurement. In the QuILT, students observed the time evolution of the wavefunction after the energy measurement and found that the system stays in the stationary state in which it collapsed $\left(\left|\psi_{1}\right\rangle\right.$ or $\left.\left|\psi_{2}\right\rangle\right)$, as shown in Figure 5.6(b) and (c), instead of going back to the initial state which is a linear superposition of these states. Students predict and then perform the same sequence of activities with a more general state 
$|\Psi\rangle=\sum A_{n}\left|\psi_{n}\right\rangle$ in which more than two coefficients are non-zero. They learn that the wavefunction in this superposition state keeps changing shape with time but the system collapses to one of the energy eigenstates and remains there after the measurement of energy. Students learn that while the measurement instantaneously collapses the wavefunction, the wavefunction after the measurement evolves in time in a deterministic manner according to the TDSE. Moreover, comparison of the time evolution of an energy eigenstate $\left|\psi_{n}\right\rangle$ (after the measurement) and a general state which is a linear superposition of stationary states (before the measurement) helps build intuition about the meaning of stationary states and non-stationary states.
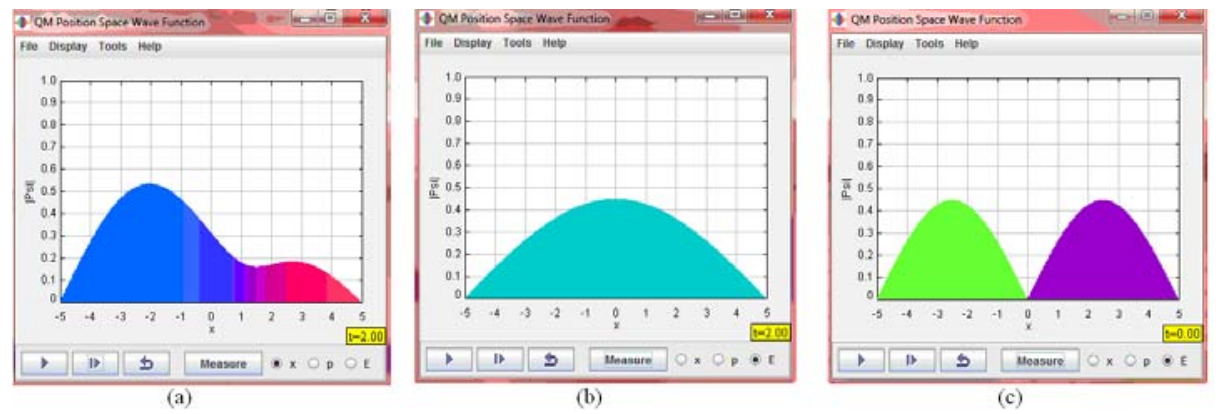

Figure 5.6 Energy measurement on a superposition state with two energy eigenstate components after time $\mathbf{t}>0$. (a) is the superposition state before the energy measurement. (b) and (c) are the two possible outcomes of the quantum measurement.

\subsubsection{Position Measurement}

Many students held the misconception that, after the position measurement, the position eigenstate does not change with time and the system is stuck in a position eigenstate. In the QuILT, students are asked to use the simulation after their initial prediction of what should happen when they perform a position measurement starting from a general state. In an ideal measurement, at the instant the position is measured, the wavefunction of the system will 
collapse to a delta function $\delta\left(x-x_{0}\right)$ (a broad peaked function in the simulation, as shown in Fig 5.7(a)) and the measured position is $x_{0}$. The position eigenstate wavefunction can be written as a linear superposition of the energy eigenstates, $\Psi(x, t=0)=\delta\left(x-x_{0}\right)=\sum_{n} A_{n} \psi_{n}(x)$. Different energy eigenstates will have their own time-dependent phase factors so that the wavefunction $\Psi(x, t)$ is not a delta function $\delta\left(x-x_{0}\right)$ except at some special times (as discussed in the appendix of this chapter). Fig 5.7(b) and (c) are the snap shots of the time evolution of the position eigenstate wavefunction.
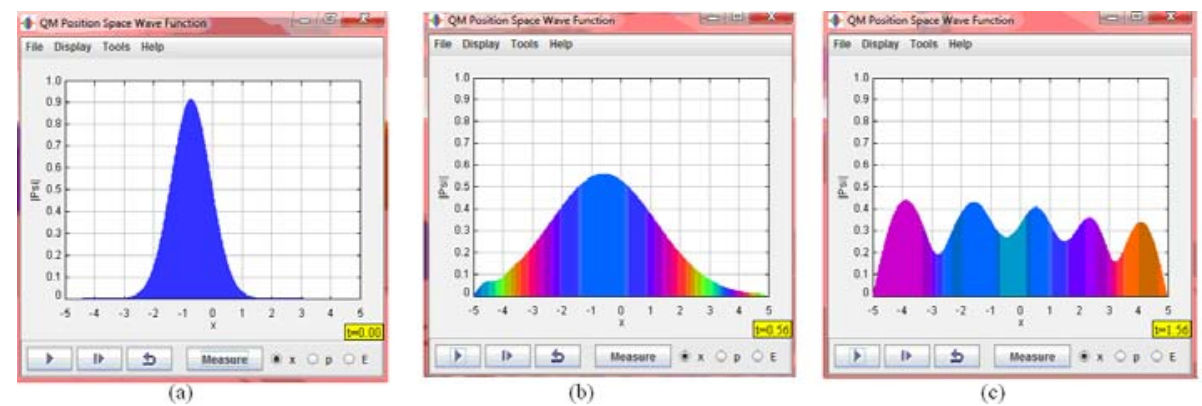

Figure 5.7 Time evolution of the position eigenstate wavefunction. (a) is the position eigenstate wavefunction right after a position measurement. (b) and (c) are the wavefunction of the system at later times after the position measurement.

Besides the pictorial representation in the simulations, the QuILT helps students learn to interpret the time evolution of the wavefunction via the TDSE and discern the central role of the Hamiltonian of the system in the evolution. The following is an example question in the QuILT:

- Given the wavefunction at time $t=0$, why is it useful to write the state of a quantum system as a superposition of energy eigenstates to find the wavefunction after time t?

Students must realize that the Hamiltonian governs the time evolution of the system according to the TDSE so the eigenstates of the Hamiltonian are special for issues related to the time evolution of the wavefunction. Help is provided at the end of the QuILT if students are struggling with these issues. 
Though the students had learned that the position eigenstate is not a stationary state, some of them still held the misconception that the position eigenstate after a position measurement would eventually return to the initial state, e.g., $\left(\left|\psi_{1}\right\rangle+\left|\psi_{2}\right\rangle\right) / \sqrt{2}$. The simulation is helpful in overcoming this difficulty. The students observe that the delta function does not remain a delta function (a broad peaked function in the simulation) as shown in Fig 5.7 (although there is revival of the delta function periodically for a 1D infinite square well). They perform a systematic analysis of the time-dependence of the wavefunction starting with a delta function to convince themselves that the system will not go back to the state right before the measurement.

\subsubsection{Time Dependence of the Measurement Probability}

It is important that students learn whether the probability of obtaining different energies or positions changes with time. For a general wavefunction $\Psi(x, t)=\sum_{n} A_{n} \psi_{n}(x) e^{-i E_{n} t / \hbar}$ at time $t$, the probability of obtaining $E_{n}$ in an energy measurement is $\left|A_{n} e^{-i E_{n} t / \hbar}\right|^{2}=\left|A_{n}\right|^{2}$, which is a constant independent of time. Let's consider the time evolution of the system after a position measurement as discussed previously. Since the delta function $\delta\left(x-x_{0}\right)$ contains non-zero coefficients $A_{n}$ for all higher energy eigenstate wavefunctions $\psi_{n}(x)(n>2)$, the probability of measuring these higher energies $\left|A_{n} e^{-i E_{n} t / \hbar}\right|^{2}$ cannot be zero. Therefore, the system cannot return to the state $\left(\left|\psi_{1}\right\rangle+\left|\psi_{2}\right\rangle\right) / \sqrt{2}$ after the position measurement no matter how long we wait.

On the other hand, when position is measured, the probability of finding the particle at $x=x_{0}$ is $\left|\Psi\left(x_{0}, t\right)\right|^{2}=\left|\sum_{n} A_{n} \psi_{n}\left(x_{0}\right) e^{-i E_{n} t / \hbar}\right|^{2}$, which depends on time. This non-trivial time- 
dependence of the probability of position measurement can be observed in the simulation since the shape of wavefunction changes with time.

In the QuILT, for the wavefunction $\left[\psi_{1}(x) e^{-i E_{1} t / \hbar}+\psi_{2}(x) e^{-i E_{2} t / \hbar}\right] / \sqrt{2}$ at time $t$, students learn to distinguish between the probability of measuring energy and position and whether each should depend on time. While the probability of measuring energy is $1 / 2$ for the ground and first excited states independent of time, the probability density for position $x$ is given by

$$
|\Psi(x, t)|^{2}=\frac{1}{2}\left[\psi_{1}^{*}(x) e^{+i E_{1} t / \hbar}+\psi_{2}^{*}(x) e^{+i E_{2} t / \hbar}\right]\left[\psi_{1}(x) e^{-i E_{1} t / \hbar}+\psi_{2}(x) e^{-i E_{2} t / \hbar}\right]
$$

which depends on time. In a sample dialogue from the QuILT below, students are asked to explain why they agree with Harry or Sally who are discussing this issue.

- Harry and Sally prepare the same initial state wavefunctions $\left[\psi_{1}(x)+\psi_{2}(x)\right] / \sqrt{2}$ from energy eigenfunctions $\psi_{1}(x)$ and $\psi_{2}(x)$ in their labs at time $t=0$. Harry measures the energy of his electron in a $1 D$ infinite square well at $t=1$ unit and Sally measures the energy of her electron in an identical $1 D$ infinite square well at time $t=3$ units.

Harry: The probability that I will measure energy $E_{n}$ is not the same as the probability that you will measure energy $E_{n}$. The probability is determined by the absolute square of the wave function, $|\Psi(x, t)|^{2}$, which depends on time.

Sally: No. The probability of measuring position depends on the absolute square of the wave function. This time we are measuring energy. The time-dependent phase factors will cancel out because only one factor $e^{-i E_{n} t / \hbar}$ can contribute in calculating the probability of measuring a particular energy $E_{n}$. Thus, the probability of obtaining $E_{n}$ will be time independent. You and I have the same probability of measuring energy $E_{n}$. 
Harry: But there will be cross terms in the square of the wave function. The phase factors do not drop out for the cross terms.

Sally: I disagree. The probability of measuring energy is determined by the square of the coefficients of each of the energy eigenfunctions $\psi_{1}(x)$ and $\psi_{2}(x)$. We do not square the entire wave function, we only square the coefficients of each energy eigenfunction and the time dependence drops out. For example, the probability of measuring energy $E_{1}$ is given by: $p\left(E_{1}\right)=\left|e^{-i E_{1} t / \hbar} / \sqrt{2}\right|^{2}$, which is time independent.

\subsubsection{Consecutive Measurements}

After students learned about how to use the "normal" time evolution of the wavefunction according to the TDSE after the measurement of a physical observable, these concepts were reinforced by asking students questions about consecutive measurements. For example, students were asked about the possible outcomes of an energy measurement after a position measurement for the state $\frac{1}{\sqrt{2}}\left(\left|\psi_{1}\right\rangle+\left|\psi_{2}\right\rangle\right)$. Some students incorrectly believed that one can only obtain energies $E_{1}$ or $E_{2}$. However, since the position measurement will collapse the system to a position eigenstate which is a superposition of all of the energy eigenstates $\left|\psi_{n}\right\rangle$ (including those corresponding to very high energies), the energy measurement that follows the position measurement could yield a very high energy value $E_{n}$. After the prediction, students use the simulation to check their prediction and find that the wavefunction could collapse to an energy eigenstate $\psi_{n}$ with $n \geq 3$ as shown in Figure 5.8. Students are also asked to calculate the probability for measuring the ground state energy. 


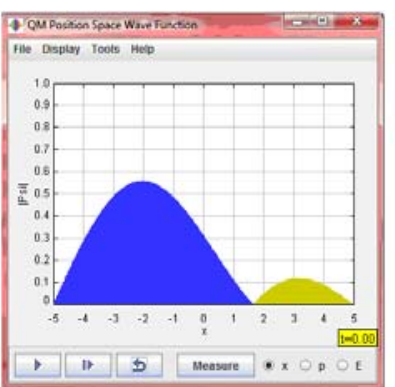

(a) Initial State

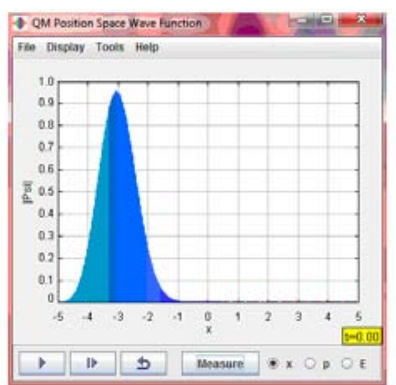

(b) Position Eigenstate

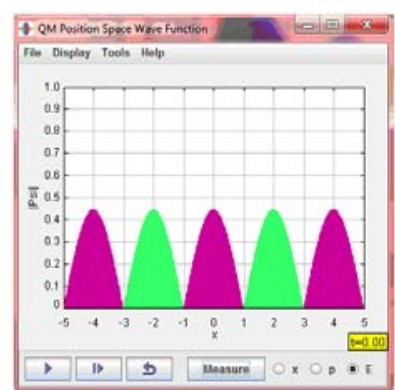

(c) Energy Eigenstate $\mathrm{n}=5$

Figure 5.8 Energy measurement after a position measurement of the initial state with only two energy eigenstate components $n=1$ and $n=2$. (a) represents the system in the initial superposition state. (b) is the state of the system right after a position measurement. (c) is a possible state (energy eigenstate $n=5$ ) of the system after the energy measurement following the position measurement in (b).

Students also predict whether the probability of measuring different values of energy depends on whether we wait for a certain time after the position measurement. When students earlier learned about the time dependence of the measurement probability, they learned that the probability of obtaining a particular energy value was independent of time and that no matter how long one waited after the position measurement, the system would not go back to the initial state before position was measured. These concepts about the "normal" time evolution according to the TDSE are reinforced by using the simulation. Students observe in the simulation that the wavefunction does not return to the initial state before the measurement and when they measure the energy at time $t>0$ after the position measurement, they have a significant probability of collapsing the system into a high energy eigenstate $\psi_{n}$.

Students are also asked to predict what would happen if they made two consecutive position measurements quickly so that the wavefunction does not have the time to evolve vs. waiting for some time before making the second measurement. Students then use simulation to check their prediction. They find that when the second measurement was made immediately after the first measurement, the particle is found approximately at the same position since the 
wavefunction does not have time to evolve. On the other hand, the wavefunction would not be a peaked delta function if we waited for a reasonable time before performing the second measurement and we can find the particle at a different position. The simulation provides the flexibility of stopping or starting the time evolution at any point (or even stepping through timeevolution slowly) so that students note the differences between the consecutive position measurements performed in quick succession as shown in Figure 5.9(a) and (b) vs. slowly as shown in Figure 5.9(c) and (d).

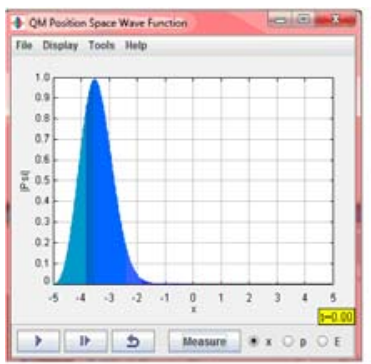

(a) state at $\mathrm{t}=0$

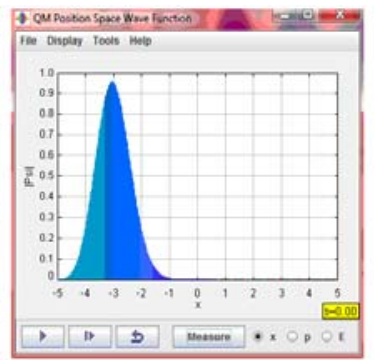

(b) measurment at $\mathrm{t}=0$

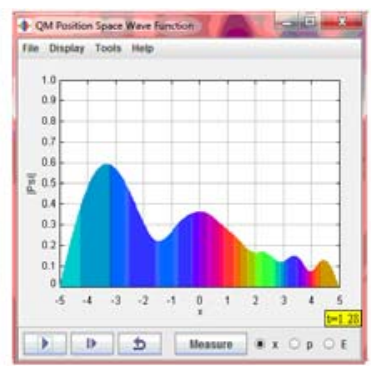

(c) state at $\mathrm{t}>0$

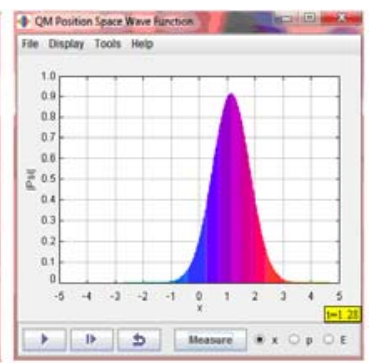

(d) measurement at $\mathrm{t}>0$

Figure 5.9 Consecutive position measurement in quick succession (a and b) /after waiting for some time (c and d)

\subsection{PRELIMINARY EVALUATION}

We designed a pre-test and a post-test to assess some issues related to measurement after the traditional instruction, after concept tests related to measurement (pre-test) and after working on the measurement QuILT (post-test). To eliminate any possible differences in the difficulty levels of the pretest and the posttest, we divided the tests into two versions, i.e., Test A and Test B. Test A and Test B both had two multiple choice questions (Q1 and Q2) and four open-ended questions (Q3-Q6). We mixed the Test A and Test B when we distributed the pre-tests and posttests to students in both the comparison group and the experimental group. Similarly, in the 
experimental group, students who obtained Test A in the pretest were given Test B in the posttest and vice versa.

The comparison group students only had traditional lectures in class and regular homework problems from the textbook. The class average was $26 \%$ including both Test A and Test B. The experimental group students had been using the concept tests as a peer instruction tool in class since the first day of the semester. The pretest was given to the students after the lecture and the average was 68\%. The experimental group students were given the QuILT as homework after being administered the pretest in class. When they turned in the QuILT as homework, they were administered the post test. Their post test average score was $91 \%$.

Table 5.1 The pre-test and post-test scores on each question of Test A. The concepts involved in each question are shown in italic.

\begin{tabular}{|c|c|c|c|}
\hline \multirow[t]{3}{*}{ Test A } & $\begin{array}{l}\text { Comparison } \\
\text { Group (15) }\end{array}$ & $\begin{array}{c}\text { Experimental Group } \\
\text { Pre-test (6) }\end{array}$ & $\begin{array}{c}\text { Experimental Group } \\
\text { Post-test (7) }\end{array}$ \\
\hline & Traditional & Lecture \& Concept & Lecture \& Concept \\
\hline & Lecture Only & $\underline{\text { Test }}$ & $\underline{\text { Test \& QuILT }}$ \\
\hline \multirow{2}{*}{ Q1 } & \multicolumn{3}{|c|}{ whether a wavefunction is an energy eigenstate } \\
\hline & $13 \%$ & $67 \%$ & $71 \%$ \\
\hline \multirow{2}{*}{ Q2 } & \multicolumn{3}{|c|}{ energy measurement outcomes of a superposition state } \\
\hline & $40 \%$ & $83 \%$ & $100 \%$ \\
\hline \multirow{2}{*}{ Q3 } & \multicolumn{3}{|c|}{ sketch the shape of a position eigenstate and find the probability } \\
\hline & $37 \%$ & $83 \%$ & $86 \%$ \\
\hline \multirow{2}{*}{ Q4 } & \multicolumn{3}{|c|}{ probability of energy measurement } \\
\hline & $3 \%$ & $63 \%$ & $79 \%$ \\
\hline \multirow{2}{*}{ Q5 } & \multicolumn{3}{|c|}{ consecutive position measurement after position measurement } \\
\hline & $37 \%$ & $71 \%$ & $100 \%$ \\
\hline \multirow{2}{*}{ Q6 } & \multicolumn{3}{|c|}{ consecutive energy measurement after energy measurement } \\
\hline & $53 \%$ & $58 \%$ & $86 \%$ \\
\hline
\end{tabular}


To analyze students' understanding of different concepts and principles in quantum measurement, we calculated the percentage of correct responses for each question in Test A and Test B as listed in Table 5.1 and Table 5.2. The numbers in the brackets represent the number of students who answered that question. The concepts involved in each question are also shown in the Tables.

Table 5.2 The pre-test and post-test scores on each question of Test B. The concepts involved in each question are shown in italic.

\begin{tabular}{|c|c|c|c|}
\hline \multirow[t]{3}{*}{ Test B } & $\begin{array}{l}\text { Comparison } \\
\text { Group (10) }\end{array}$ & $\begin{array}{c}\text { Experimental Group } \\
\text { Pre-test (7) }\end{array}$ & $\begin{array}{c}\text { Experimental Group } \\
\text { Post-test (5) }\end{array}$ \\
\hline & $\underline{\text { Traditional }}$ & Lecture \& Concept & Lecture \& Concept \\
\hline & Lecture Only & $\underline{\text { Test }}$ & Test \& QuILT \\
\hline \multirow{2}{*}{ Q1 } & \multicolumn{3}{|c|}{ what state will the system be in after a quantum measurement } \\
\hline & $50 \%$ & $57 \%$ & $80 \%$ \\
\hline \multirow{2}{*}{ Q2 } & \multicolumn{3}{|c|}{ what is a stationary state } \\
\hline & $0 \%$ & $57 \%$ & $100 \%$ \\
\hline \multirow{2}{*}{ Q3 } & \multicolumn{3}{|c|}{ energy measurement outcomes and probabilities } \\
\hline & $15 \%$ & $93 \%$ & $100 \%$ \\
\hline \multirow{2}{*}{ Q4 } & \multicolumn{3}{|c|}{ sketch the shape of an energy eigenstate } \\
\hline & $35 \%$ & $86 \%$ & $100 \%$ \\
\hline \multirow{2}{*}{ Q5 } & \multicolumn{3}{|c|}{ consecutive position measurement after energy measurement } \\
\hline & $10 \%$ & $36 \%$ & $100 \%$ \\
\hline \multirow{2}{*}{ Q6 } & \multicolumn{3}{|c|}{ consecutive energy measurement after position measurement } \\
\hline & $0 \%$ & $64 \%$ & $100 \%$ \\
\hline
\end{tabular}

From Q1 in Test A and Q2 in Test B, we observe that the concept tests in class resolved many of the students' difficulties related to the concept of stationary states. Q4 in Test A and Q3 in Test B suggest that students had understood the basic principle of the projection method when calculating the measurement probabilities. For the questions related to the time development of 
the wavefunction after a measurement, e.g., Q2 in Test B and Q6 in both Test A and B, the QuILT led students to a better performance compared to when they used the concept tests only. Also, the QuILT helped the students to correctly answer the questions related to consecutive measurements. Due to the limitation of sample size, individual performance might affect the average score on each question. However, the effectiveness of the concept tests and QuILTs in improving students' performance is reflected by the significant difference in the performance of the experimental group and the comparison group.

\subsection{SUMMARY}

We find that students have common difficulties with concepts related to quantum measurement. In particular, many students were unclear about the difference between energy eigenstates and eigenstates of other physical observables and what happens to the state of the system after the measurement of an observable. Students also had difficulty in distinguishing between individual measurements and expectation value. They struggled to distinguish between the measured value, the probability of measuring it and the expectation value. Students often did not think of the expectation value of an observable as an ensemble average of a large number of measurements on identically prepared systems but rather thought of it as a mathematical procedure where an operator is sandwiched between the same bra and ket states (the state of the system). Students were also confused about whether the system is stuck in the state in which it collapsed right after the measurement or whether it goes back to the state before the measurement was performed. In general, students struggled with issues related to the time evolution of the wave function after the measurement. 
We developed a research-based QuILT and concept tests to improve students' understanding of quantum measurement concepts. Both these learning tools keep students actively engaged in the learning process. They provide a guided approach to bridge the gap between the quantitative and conceptual issues related to quantum measurement and help students connect different concepts and build a good knowledge structure. The instructors could either use the QuILT as an in-class tutorial or assign it as homework with the pre-test and posttest given in the class. Our preliminary results show that the QuILT and concept tests significantly improve students’ understanding of quantum measurement.

\subsection{APPENDIX}

For a 1-D infinite square well model, the allowed energies are labeled by integers $n$, i.e., $E_{n}=\frac{n^{2} \pi^{2} \hbar^{2}}{2 m a^{2}}$. For any initial state $\Psi(x, t=0)=\sum_{n} A_{n} \psi_{n}$ where $A_{n}$ are suitable expansion coefficients, the wavefunction at time $t$ is

$$
\Psi(x, t)=\sum_{n} A_{n} \psi_{n} e^{-i E_{n} t / \hbar} \equiv \sum_{n} A_{n} \psi_{n} \exp \left(-i \frac{\pi^{2} \hbar}{2 m a^{2}} n^{2} t\right)
$$

At particular equally spaced times $t$ which satisfies $\frac{\pi^{2} \hbar}{2 m a^{2}} t=2 N \pi(N=1,2,3, \ldots)$, the exponential terms in the wave function are all equal to one so the wavefunction goes back to the initial state. So we do have $100 \%$ probability of measuring the same position if we make the second measurement at those particular times. However, in the question asked, we were evaluating whether students know that the position eigenstate is not a stationary state. If a student showed understanding of the time evolution of the wave function and mentioned that the probability of 
obtaining the initial value of position with $100 \%$ probability was repeated at special times only, it would be correct. However, no student explained the time evolution process in such a way after the position measurement. Some students incorrectly noted that the wavefunction goes back to the initial state before the measurement was made. What these students meant was that if the wavefunction was in a linear superposition of the ground and first excited states of the system, then some time after the measurement of energy or position, the wavefunction will go back to the initial linear superposition.

\subsection{CHAPTER REFERENCES}

Singh, C. (2006). “Assessing and improving student understanding of quantum mechanics”, in 2006 AIP Conference, Vol. 818, pp. 69-72

Singh, C. (2007). "Student difficulties with quantum mechanics formalism", in 2007 AIP Conference, Vol. 883, pp. 185-188

Singh, C. (2008a). "Student understanding of quantum mechanics at the beginning of graduate instruction”, Am. J. Phys, 76(3), 277-287

Singh, C. (2008b). "Interactive learning tutorials on quantum mechanics”, Am. J. Phys, 76(4), 400-405

Belloni, M. and Christian, W. (2003). "Physlet for quantum mechanics”, Comput. Sci. Eng, 5, 90-96

Belloni, M., Christian, W. and Cox, A. (2006). Physlet Quantum Physics. Upper Saddle River, NJ: Prentice Hall. 


\title{
6.0 IMPROVING STUDENTS' UNDERSTANDING OF QUANTUM MECHANICS VIA THE STERN-GERLACH EXPERIMENT
}

\begin{abstract}
6.1 ABSTRACT
The Stern-Gerlach experiment (SGE) has played a central role in the discovery of spin angular momentum. It can also play a pivotal role in teaching the formalism of quantum mechanics using a concrete example involving a finite-dimensional Hilbert space. Using this context, students can learn about how to prepare a specific quantum state starting from an arbitrary state, issues related to the time evolution of the wave function, and quantum measurement. It can also be exploited to teach students about the distinction between the physical space where one performs the experiment and the Hilbert space where the state of the system lies and how the information about the state of the system in the Hilbert space can be exploited to interpret the possible outcomes of the experiment in the physical space. Students can learn the advantages of choosing an appropriate basis to make suitable predictions about the outcomes of experiments with different arrangements of Stern-Gerlach devices. This experiment can also help students understand that an ensemble of identically prepared systems, e.g., one in a linear superposition of two stationary states, is not the same as a mixture, e.g., in which half of the systems are in one stationary state and the other half are in the other stationary state. Here, we discuss investigation of students' difficulties about the Stern-Gerlach experiment by giving written tests and
\end{abstract}


interviewing advanced undergraduate and graduate students in quantum mechanics courses. We also discuss preliminary data from two quantum mechanics courses that suggest that a Quantum Interactive Learning Tutorial (QuILT) related to the Stern-Gerlach experiment is helpful in improving students' understanding of these concepts.

\subsection{INTRODUCTION}

In the SGE, a particle with mass, spin and/or orbital angular momentum (a particle with a magnetic dipole moment) is sent through a Stern-Gerlach Apparatus (SGA) with a non-uniform magnetic field. With an appropriate gradient of the external magnetic field, different components of the angular momentum in the wave function can be spatially separated by coupling them with different linear momenta. By using suitable measurement devices (e.g., detectors at appropriate locations in the path of the beam), we can use the SGE to prepare a quantum state which is different from the initial state before the particle entered the SGA. The knowledge deficiencies related to the SGE discussed in the next section can be broadly divided into three levels in order of increasing difficulty in overcoming them: (I) lack of knowledge of relevant concepts, (II) knowledge that cannot be interpreted correctly, (III) knowledge that is interpreted at the basic level but cannot be used to draw inferences in specific situations (Singh 2001).

The SGE QuILT is based upon research on students' difficulties in learning quantum mechanics. It strives to build on students' prior knowledge, actively engages them in the learning process and helps them build links between the abstract formalism and conceptual aspects of quantum physics without compromising the technical content. The QuILT uses a guided inquiry method of learning and the various sections build on what the students did in the previous 
sections to help them develop a robust knowledge structure. As students progress through the QuILT, they first make predictions about what should happen in various situations and then they are given guidance and support to reason through the situations appropriately and assimilate and accommodate productive ideas into their knowledge structure (McDermott et al. 2002). The SGE QuILT creates an active learning environment in which students will directly confront their misconceptions. At various stages of concept development, the SGE QuILT often exploits computer-based visualization tools. Often these tools cause a cognitive conflict if students' initial prediction and their observations do not match. In that case, students themselves realize that there is some inconsistency in their reasoning. Then, providing students appropriate guidance and support via the guided inquiry approach used in the QuILT is an effective strategy to help them build a robust knowledge structure.

\subsection{INVESTIGATION OF STUDENTS’ DIFFICULTIES WITH THE STERN- GERLACH EXPERIMENT}

The investigation of difficulties was carried out by administering written surveys to more than two hundred physics graduate students and advanced undergraduate students enrolled in quantum mechanics courses and by conducting individual interviews with a subset of students. The individual interviews were carried out using a think-aloud protocol to better understand the rationale for their responses before, during and after the development of different versions of the SGE QuILT and the corresponding pre-test and post-test (Chi 1994). During the semi-structured interviews, students were asked to verbalize their thought processes while they answered questions either as separate questions before the preliminary version of the QuILT was 
developed or as a part of the QuILT. Students were not interrupted unless they remained quiet for a while. In the end, we asked them for clarification of the issues they had not made clear earlier. Some of these interviews involved asking students to predict what should happen in a particular situation, having them observe what happens in a simulation, and asking them to reconcile the differences between their prediction and observation. After each individual interview with a particular version of the QuILT (along with the pre-test and post-test administered), modifications were made based upon the feedback obtained from students' performance on the QuILT (if students got stuck at a particular point and could not make progress from one question to the next with the hints already provided, suitable modifications were made), the pre-test and the post-test.

\subsubsection{Difficulty in Distinguishing between the Physical Space and Hilbert Space}

Using quantum theory, one can interpret the outcome of experiments performed, e.g., in three dimensional (3D) laboratory or physical space by making connection with an abstract Hilbert space (state space) in which the state of the quantum system or wavefunction lies. The physical observables that one measures in the laboratory correspond to Hermitian operators in the Hilbert space whose eigenstates span the Hilbert space. Knowing the initial wavefunction and the Hamiltonian of the system allows one to determine the time-evolution of the wavefunction unambiguously and the measurement postulate can be used to determine the possible outcomes of individual measurements of an observable and their ensemble averages (expectation values).

It is difficult for many students to distinguish between vectors in the 3D laboratory space

and states in Hilbert space. For example, $S_{x}, S_{y}$ and $S_{z}$ denote the orthogonal components of the spin angular momentum vector of an electron in the 3D space, each of which is a physical 
observable that can be measured in the laboratory. However, the Hilbert space corresponding to the spin degree of freedom for a spin-1/2 particle is two dimensional (2D). In this Hilbert space, $\hat{S}_{x}, \hat{S}_{y}$ and $\hat{S}_{z}$ are operators whose eigenstates span the 2D space. Thus, the eigenstates of $\hat{S}_{x}$ are vectors which span the 2D space and are orthogonal to each other (but not orthogonal to the eigenstates of $\hat{S}_{y}$ and $\hat{S}_{z}$ ). If the electron is in a magnetic field with the field gradient in the $z$ direction in the laboratory (3D space) as in the Stern-Gerlach experiment, the magnetic field is a vector field in the 3D space but not in the 2D Hilbert space. It does not make sense to compare vectors in the $3 \mathrm{D}$ space with the vectors in the $2 \mathrm{D}$ space as in statements such as "the magnetic field gradient is perpendicular to the eigenstates of $\hat{S}_{x}$ ”. In fact, even $L=1$ orbital angular momentum states, which are vectors in a 3D Hilbert space, do not live in the 3D laboratory space. Unfortunately, these distinctions are difficult for students to make and such difficulties were frequently observed in response to the survey questions and during the individual interviews. These difficulties are discussed below in the context of the Stern-Gerlach experiment.

\subsubsection{Difficulty in Determining the Pattern on the Screen with Particles in Different Spin States Passing through a SGA}

Two questions we have asked the first year physics graduate students and advanced undergraduate students for several years related to the SGE in written tests and interviews are questions (1) and (2) in the Appendix (section 6.8). In one version of these questions, neutral silver atoms were replaced with electrons and students were told to ignore the Lorentz force.

In question (1) in the Appendix, students have to realize that the magnetic field gradient in the $-\mathrm{z}$ direction would impart a spin-dependent momentum to the particle and one should 
observe two spots on the phosphor screen owing to the splitting of the beam along the z-direction due to the particle's spin components corresponding to the $|\uparrow\rangle_{z}$ and $|\downarrow\rangle_{z}$ states. All responses in which students noted that there will be a splitting along the z-direction were considered correct even if they did not explain their reasoning. Only $41 \%$ of the more than 200 graduate students from different universities enrolled in a quantum mechanics course provided the correct response. These students were given this question as a part of a survey at the beginning of graduate level quantum mechanics instruction. Many students thought that there will only be a single spot on the phosphor screen. During the interviews conducted with a subset of students, they were often confused about the origin of the spin-dependent momentum imparted to the particle. The same question was given to 35 undergraduate students in two different classes immediately after instruction in the SGE. These students obtained $80 \%$ on this question, which is significantly better than the performance of the graduate students before instruction in the graduate-level course. It appears that many of the first year graduate students enrolled in the graduate level quantum mechanics course who took the survey had forgotten about the SGE. Moreover, discussions with some of the graduate students suggest that they had learned it only in the context of a modern physics course which was qualitative.

Question (2) in the Appendix is challenging because students have to realize that since the magnetic field gradient is in the -x direction, the basis must be chosen to be the eigenstates of $\hat{S}_{x}$ to readily analyze how the SGA will affect the spin state. Here, the initial state, which is an eigenstate of $\hat{S}_{z},|\uparrow\rangle_{z}$, can be written as a linear superposition of the eigenstates of $\hat{S}_{x}$, i.e.,

$$
\left.|\uparrow\rangle_{z}=\frac{1}{\sqrt{2}}(\uparrow\rangle_{x}+|\downarrow\rangle_{x}\right)
$$


The magnetic field gradient in the -x direction will couple the $|\uparrow\rangle_{x}$ and $|\downarrow\rangle_{x}$ components in the incoming spin state $|\uparrow\rangle_{z}$ with oppositely directed x-components of the linear momentum and will cause two spots on the phosphor screen separated along the x-axis.

Only $23 \%$ of the more than 200 graduate students in a survey at the beginning of instruction provided the correct response. The performance of 35 undergraduate students from two different classes who were given this question immediately after traditional instruction in SGE was only somewhat better (39\%). Some undergraduate and graduate students were interviewed individually to better understand the reasoning behind their response. In some of these interviews, we asked students to predict the outcome of these experiments and then showed them what actually happens in a simulation and asked them to reconcile the differences between the observation and prediction. This task turned out to be extremely difficult for students. The most common difficulty in Question (2) was assuming that since the spin state is $|\uparrow\rangle_{z}$, there should not be any splitting as shown in Figure 6.1.

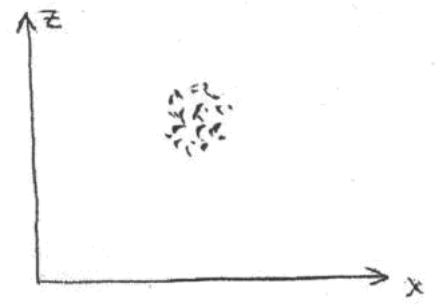

Student's comment: The inner product

between the B field and the $|\uparrow\rangle_{z}$ will

be zero because they are orthogonal.

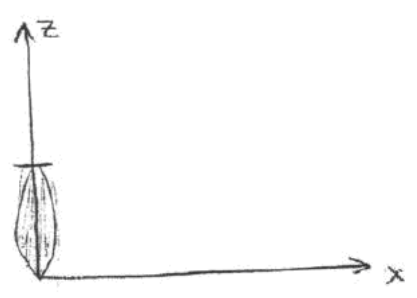

Student's comment: $\hat{S}_{z}|\uparrow\rangle_{z}=|\uparrow\rangle_{z}$

Similarly, however here $\psi=|\uparrow\rangle_{z}$ has

only one eigenvector. Thus $H \psi \sim|\uparrow\rangle$

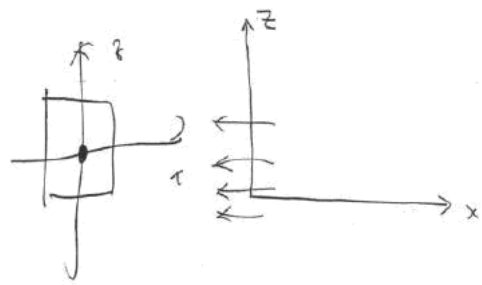

Student's comment: We have one spot on the origin. For this time, the force acts on the electron is $F_{x}=-\mu_{x} \frac{\partial B_{x}}{\partial x}$ and $\mu_{x}=0$ so there's actually no force acting on the electron.

Figure 6.1 Three sample responses in which students provided incorrect explanations for why there should be one spot instead of two in question (2) in the Appendix. The students' comments with each figure are typed for clarity. 
Many students explained their reasoning by claiming that since the magnetic field gradient is in the $-x$ direction but the spin state is along the $z$-direction, they are orthogonal to each other, and therefore there cannot be any splitting of the beam. Student responses suggest that they were incorrectly connecting the gradient of the magnetic field in the 3D space with the "direction" of state vectors in the Hilbert space. Several students in question (2) drew a monotonically increasing function. Some of them incorrectly believed that the spin state in this situation will get pulled in one direction because the magnetic field gradient is in a certain direction (see Figure 6.2). Asking the interviewed students explicitly about whether they could consider a basis that may be more appropriate to analyze this problem was rarely helpful.

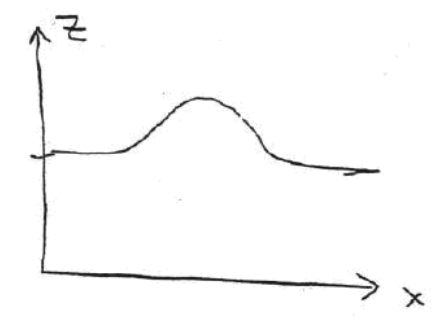

Student's comment: The spin state reacts to the field; this time there is only one state, such that the beam bends only in one direction. Because spin up exists, it will be in the $+z$ (direction).

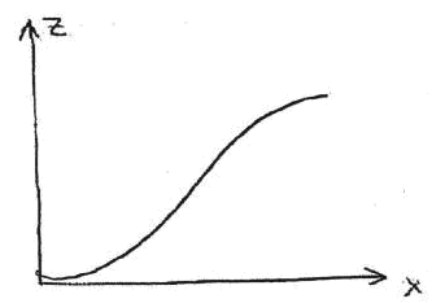

Student's comment: The state goes along with the gradient.

Figure 6.2 Two sample responses in which students provided incorrect explanations for why the state/beam will bend as shown in response to the magnetic field gradient in question (2) in the Appendix. The students' comments with each figure are typed for clarity.

One student drew the diagram shown in Figure 6.3 and described Larmor precession of spin but did not mention anything about the spin-dependent momentum imparted to the particle due to the non-uniform magnetic field as in the SGE. Written responses and interviews suggest that many students were unclear about the fact that in a uniform external magnetic field, the spin will only precess (if not in a stationary state) but in a non-uniform magnetic field as in the SGE, 
there will be a spin-dependent momentum imparted to the particle that may spatially separate the components of the spin angular momentum in the wave function under suitable conditions.

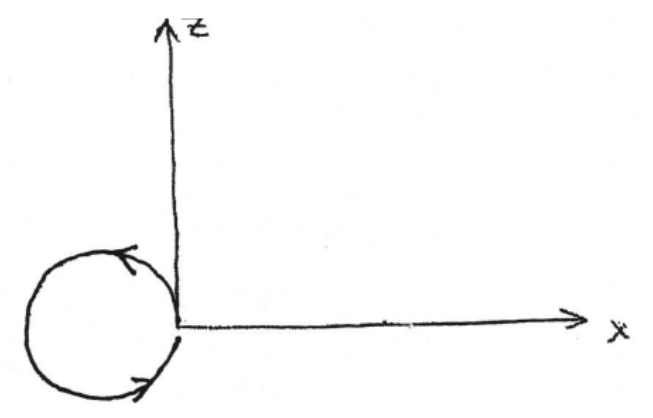

Figure 6.3 A diagram drawn by a student showing the Larmor precession of spin in response to question (2) in the Appendix.

\subsubsection{Larmor Precession of Spin involves Precession in Physical Space}

We note that the student who drew Figure 6.3 incorrectly believed that spin is due to motion in real space. When he was reminded that the question was not about the dynamics (as suggested by the arrows drawn by the student to show the direction of precession) but about the pattern observed on the screen, he incorrectly claimed that the pattern on the screen would be a circle due to the precession of the spin in the magnetic field. Similar to the difficulty of this student, we have found that many students have difficulty realizing that spin is not an orbital degree of freedom and we see two spots on the screen in questions (1) and (2) in the Appendix related to the SGE because of the coupling of the spin degree of freedom with the orbital degree of freedom (e.g., the linear momentum). 


\subsubsection{Difficulty with State Preparation}

The preparation of a specific quantum state may be challenging to achieve in the laboratory but it is relatively easy to conceptualize theoretically at least in a 2D Hilbert space with the SGE. We find that the students have difficulty with the preparation of a specific quantum state even in a 2D Hilbert space. Students were asked questions related to state preparation using SGA in both written tests and interviews, e.g., question (8) in the Appendix.

A possible correct response would be to pass the initial beam through a SGA with a magnetic field gradient in the $\mathrm{x}$ or $\mathrm{y}$ direction and block one component of the spatially separated beam that comes out of the SGA before passing it through another SGA with its field gradient in

the z-direction. One can then block the $|\uparrow\rangle_{z}$ component with a detector and obtain a beam in the spin state $|\downarrow\rangle_{z}$.

Out of 17 first year graduate students enrolled in quantum mechanics who had instruction in the SGE, 82\% provided the correct response to question (8) in the Appendix. However, only $30 \%$ of undergraduate students after traditional instruction provided the correct response. Interviews suggest that students had great difficulty thinking about how to choose an appropriate basis to facilitate the analysis of what should happen after particles in a given spin state were sent through a SGA with a particular magnetic field gradient. 


\subsubsection{Difficulty in Differentiating between a Superposition and a Mixture}

We also asked students to think of a strategy to distinguish between a superposition in which all particles are in state $\frac{1}{\sqrt{2}}\left(|\uparrow\rangle_{z}+|\downarrow\rangle_{z}\right)$ from a mixture in which half of the particles are in state $|\uparrow\rangle_{z}$ and the other half are in state $|\downarrow\rangle_{z}$ as in question (9) in the Appendix.

This question was very difficult for most students. One strategy for distinguishing between the superposition and the mixture given is to pass each of them one at a time through a SGA with the field gradient in $-x$ direction. Then, since $\frac{1}{\sqrt{2}}\left(|\uparrow\rangle_{z}+|\downarrow\rangle_{z}\right)$ is $|\uparrow\rangle_{x}$, it will completely go out through the upper-channel after passing through a SGA with a negative $\mathrm{x}$ gradient (SGX-). On the other hand, the equal mixture of $|\uparrow\rangle_{z}$ and $|\downarrow\rangle_{z}$ will have an equal probability of registering at the detectors in the lower and upper channels after the SGX-because these states can be written as $\frac{1}{\sqrt{2}}\left(|\uparrow\rangle_{x} \pm|\downarrow\rangle_{x}\right)$ in terms of the eigenstates of $\hat{S}_{x}$ and will become spatially separated after passing through the SGX-.

Out of 17 first year graduate students enrolled in quantum mechanics who had instruction in the SGE only $24 \%$ provided the correct response to this question. In an undergraduate course in which the instructor had discussed similar problems with students before giving them this question, 31\% provided the correct response after the traditional instruction. One student incorrectly noted: "Since the probability for an atom in the beam A to be in either state $|\uparrow\rangle_{z}$ or $|\downarrow\rangle_{z}$ is $1 / 2$, I can't distinguish it from B.” Another incorrect response emphasized differences in coupling of the spin angular momentum with the linear momentum: “The atoms in beam A will 
have their spin coupled to the z-component of their momentum. The other beam's atoms, however, will not have $P_{z}$ coupled to $S_{z}$." Some students who believed that it is possible to separate a mixture from a superposition state using SGA provided incorrect reasoning. Figure 6.4 provides two such examples in which students first let each of the beams pass through a SGA with a magnetic field gradient in the $z$-direction.

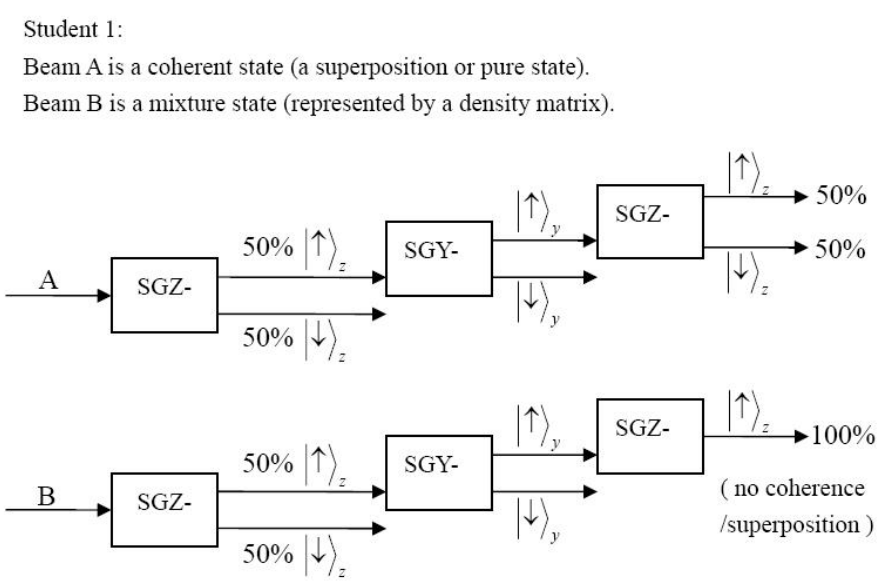

Student 2:

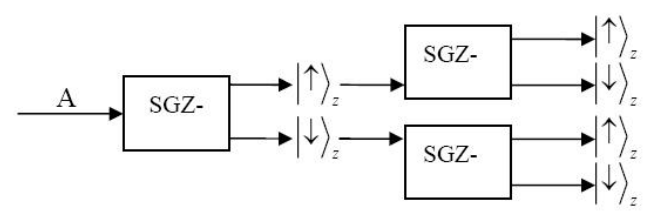

Figure 6.4 Examples of two graduate students' responses to question (9). The students' responses are typed for clarity.

\subsection{SGE QUILT: WARM-UP AND HOMEWORK}

As discussed in the introductory section, the SGE QuILT builds on the prior knowledge of students and was developed based on the difficulties found via written surveys and interviews. The QuILT development went through a cyclical iterative process which includes the following 
stages: (1) Development of the preliminary version based upon theoretical analysis of the underlying knowledge structure and research on students' difficulties, (2) Implementation and evaluation of the QuILT by administering it individually to students, measuring its impact on student learning and assessing what difficulties remained, (3) refinement and modification based upon the feedback from the implementation and evaluation. When we found that the QuILT was working well in individual administration and the post-test performance was significantly improved compared to the pre-test performance, it was administered in quantum mechanics classes.

The SGE QuILT begins with a warm-up exercise and includes homework questions that students work on before and after working on the QuILT, respectively. The warm-up exercise discusses preliminary issues such as why there is only a torque on the magnetic dipole in a uniform magnetic field but also a "force” in a non-uniform magnetic field (or more precisely, a momentum is imparted to the particle due to its angular momentum as in the SGE). It also helps students understand that the divergence of the magnetic field being zero according to the Maxwell's equation implies that the gradient of the magnetic field cannot be non-zero only in one direction and if we choose the gradient to be non-zero in two orthogonal directions and also apply a strong uniform magnetic field in one of those directions, the rapid Larmor precession will make the average force in one of the directions zero. That way we can only focus on the magnetic field gradient in a particular direction for determining its effect on the spin state after passing through the SGE.

The warm-up exercise also discusses how the overall wavefunction of the quantum system includes both the spatial and spin parts of the wavefunction. For simplicity, students are asked to assume that before passing through a Stern Gerlach device with the field gradient in the 
z-direction (SGZ) at time $t=0$, the spatial wave function $\psi(x, y, z)$ is a Gaussian localized near $(x, y, z)=(0,0,0)$ and the spatial and spin parts of the wave function are not entangled. Therefore, the overall wave function which includes both the spatial and spin parts can be written as $\Psi(t=0)=($ orbital part $) \times($ spin part $)$, i.e., $\Psi(t=0)=\psi(x, y, z)|\chi\rangle$. Students are guided via a series of questions including the following:

A silver atom in the spin state $\chi(t=0)=\left(a|\uparrow\rangle_{z}+b|\downarrow\rangle_{z}\right)$ passes through a SGZ- with a non-uniform magnetic field $\vec{B}=C_{0} z \hat{k}$ from time $t=0$ to $t=T$. Which one of the following is the wave function at time $t=T$ when the atom just exits the magnetic field? Assume that the atom is in the SGZ- for a short time so that there is no change in the $x, y, z$ coordinates. (Hint: The time development of each stationary state is via an appropriate term of the type $e^{ \pm i E_{ \pm} t / \hbar}$ )
A. $\Psi(T)=a \phi_{+}|\uparrow\rangle_{z}+b \phi_{-}|\downarrow\rangle_{z}$, where $\phi_{ \pm}(x, y, z)=e^{ \pm C_{0} \gamma \cdot z T / 2} \psi(x, y, z)$
B. $\Psi(T)=\phi_{+}(x, y, z)\left(a|\uparrow\rangle_{z}+b|\downarrow\rangle_{z}\right)$
C. $\Psi(T)=\psi(x, y, z)\left(a|\uparrow\rangle_{z}+b|\downarrow\rangle_{z}\right)$
D. None of the above.

Students further learn that in the wavefunction at time $t=\mathrm{T}, \Psi(T)=a \phi_{+}|\uparrow\rangle_{z}+b \phi_{-}|\downarrow\rangle_{z}$ the spatial and spin parts of the wave functions are "entangled" because spin and orbit cannot be factorized (i.e., cannot be written in the form $\Psi(T)=\psi(x, y, z)\left(a|\uparrow\rangle_{z}+b|\downarrow\rangle_{z}\right)$ ). Thus, measurement of the orbital degrees of freedom is linked to spin and vice versa. Students are told that in the future discussion in the QuILT, the spatial part of the wave function $\psi(x, y, z)$ will not be mentioned explicitly. However, they should understand that a SGA entangles the spatial and spin parts of the wave function. 
The warm-up helps students understand how the coupling of the orbital and spin degrees of freedom causes the spatial separation of various spin components of the wave function. In the warm-up, students also learn that while the different components of spin may get spatially separated after passing through a SGA, the wave function will remain in a superposition of different spin states until a measurement is made, e.g., by placing a detector in an appropriate location. For example, the wave function for a spin-1/2 particle can become spatially separated after passing through certain orientations of SGA and if a detector placed after the SGA at an appropriate location detects a particle (clicks), the wave function collapses to one state vs. when the detector does not click (in which case we have prepared the particles in a definite spin state).

In the SGE QuILT warm-up, students also learn about issues related to distinguishing between vectors in three-dimensional physical space and state vectors in Hilbert space. In this context, they learn that the magnetic field gradient in the z-direction is not perpendicular to a spin state in the Hilbert space, a common misconception among students. Students also learn about why choosing a particular basis is useful when analyzing particles going through a SGA with a particular magnetic field gradient. The SGE QuILT warm-up also helps clarify confusion about the $\mathrm{x}, \mathrm{y}$ and $\mathrm{z}$ labels used to denote the orthogonal components of a vector, e.g., in classical mechanics, and the eigenstates of different components of spin operator $\left(\hat{S}_{x}, \hat{S}_{y}\right.$ and $\left.\hat{S}_{z}\right)$ which are not orthogonal to each other.

The SGE QuILT homework extends what students have learned in the tutorial and also focuses further on issues related to quantum measurement and state preparation via SGE. One common difficulty about SGE is that students often believe that a particle passing through a SGE is equivalent to the measurement of particle's spin angular momentum. These issues are clarified in the SGE QuILT homework. 


\subsection{SGE QUILT}

As noted earlier, the SGE QuILT uses a guided inquiry-based approach in which various concepts build on each other gradually. It employs visualization tools to help students build a physical intuition about concepts related to the SGE. The Open Source Physics SPINS program (Belloni et al. 2006) was adapted as needed for the SGE QuILT. The SPINS program extends David McIntyre's open source Java applet (McIntyre 2002) by allowing simulated experiments to be stored and run easily.

One effective strategy to help students build a robust knowledge structure is to cause a cognitive conflict in students' minds such that the students themselves realize that there is some inconsistency in their reasoning and then provide them appropriate guidance and support. In the SGE QuILT, after predicting what they expect in various situations, students are asked to check their predictions using simulations. If the prediction and observations do not match, students reach a state of cognitive conflict. At that point the QuILT provides them guidance to help build a good grasp of relevant concepts and reconcile the difference between their predictions and observations.

As noted earlier, the SGE QuILT helps students learn about issues related to

measurement, preparation of a desired quantum state, e.g., $|\uparrow\rangle_{x}$, starting with an arbitrary initial state, time-development of the wave function, the difference between superposition and mixture, the difference between physical space and Hilbert space, the importance of choosing an appropriate basis to analyze what should happen in a particular situation, etc. The full version of the SGE QuILT is attached at the end of the thesis (Appendix B). Below we discuss some excerpts of the guidance provided by the SGE QuILT. In each situation, students have to first 
predict what should happen in a particular situation before guidance and hints are provided to them. As an example, students are asked questions such as the following to help them understand the state preparation, which is a central concept in being able to exploit quantum mechanics in different applications including possibly to build a quantum computer some day.

- You send silver atoms in an initial spin state $|\uparrow\rangle_{z}$ one at a time through two SGAs with magnetic field gradients as shown in Figure 6.5. Suitable detectors are placed as shown in Figure 6.5. One detector is between the two SGAs (in the lower channel) and the other after both SGAs (in the upper channel). What is the probability that a given single atom will cause the "up" detector to click after passing through this system of two SGAs?

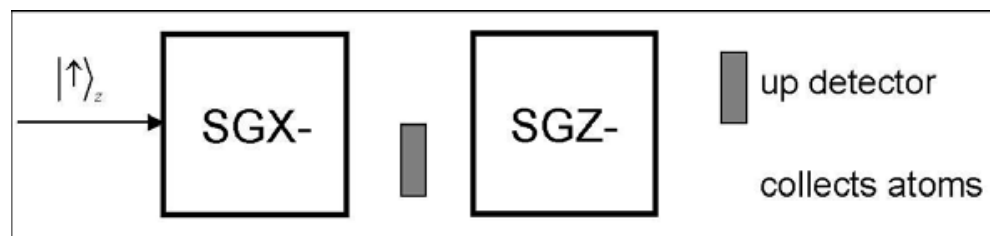

Figure 6.5 Set up for a guided example in the QuILT.

- You collect the silver atoms that are not blocked at the end after they have passed through both SGAs. Which one of the following is the spin state of the silver atom you collect at the end in the lower channel?

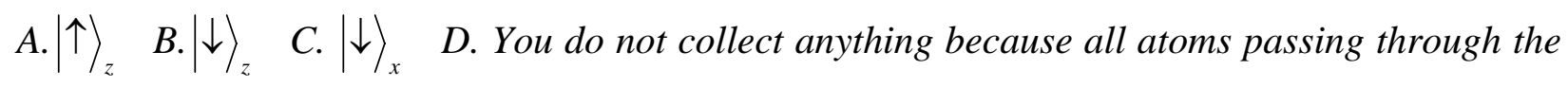
second SGA are blocked by the detector

Figure 6.6 shows a simulation constructed from the OSP SPINS (Belloni 2006) program that students work with after their initial prediction that shows that one can input $|\uparrow\rangle_{z}$ and obtain $|\downarrow\rangle_{z}$. Students again have to reconcile the difference between their prediction and observation with suitable hints. 


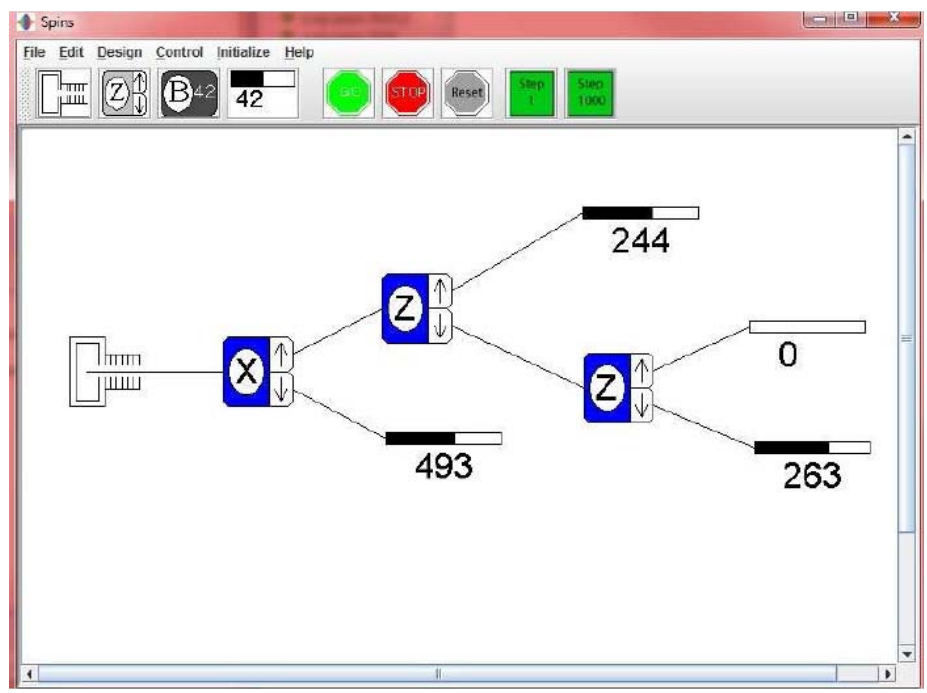

Figure 6.6 A snapshot of the simulated experiment constructed from the OSP SPINS program. This snapshot shows 493 particles are registered in the detector right after passing through the SGA with the magnetic field gradient in the negative $x$-direction (SGX-), 244 particles are registered in the detector right after the first SGA with the magnetic field gradient in the negative z-direction (SGZ-) and 263 particles are registered in the detector after the second SGZ-.

In order to help students understand that it is possible to input $|\uparrow\rangle_{z}$ through the SGAs and prepare an orthogonal state $|\downarrow\rangle_{z}$ on the way out, the QuILT also draws an analogy with the photon polarization states. Students learn that if atoms in the state $|\uparrow\rangle_{z}$ pass through a SGZ only, the state $|\downarrow\rangle_{z}$ will not be obtained on the way out. However, $|\downarrow\rangle_{z}$ is obtained in the simulated experiment in Figure 6.6 because we have inserted SGX- at an intermediate stage. Students consider the analogy with vertically polarized light passing directly through a horizontal polarizer (Figure 6.7a) vs. passing first through a polarizer at $45^{\circ}$ followed by a horizontal polarizer (Figure 6.7b). There is no light at the output if vertically polarized light passes directly through a horizontal polarizer. On the other hand, if the polarizer at $45^{\circ}$ is present, light becomes polarized at $45^{\circ}$ after the $45^{\circ}$ polarizer, which is a linear superposition of horizontal and vertical 
polarization. Therefore, some light comes out through the horizontal polarizer placed after the $45^{\circ}$ polarizer. Since the experiment with the polarizers (in the context of a photon beam not single photon) is familiar to students from introductory physics, this analogy can help students learn about the SGE using a familiar context.

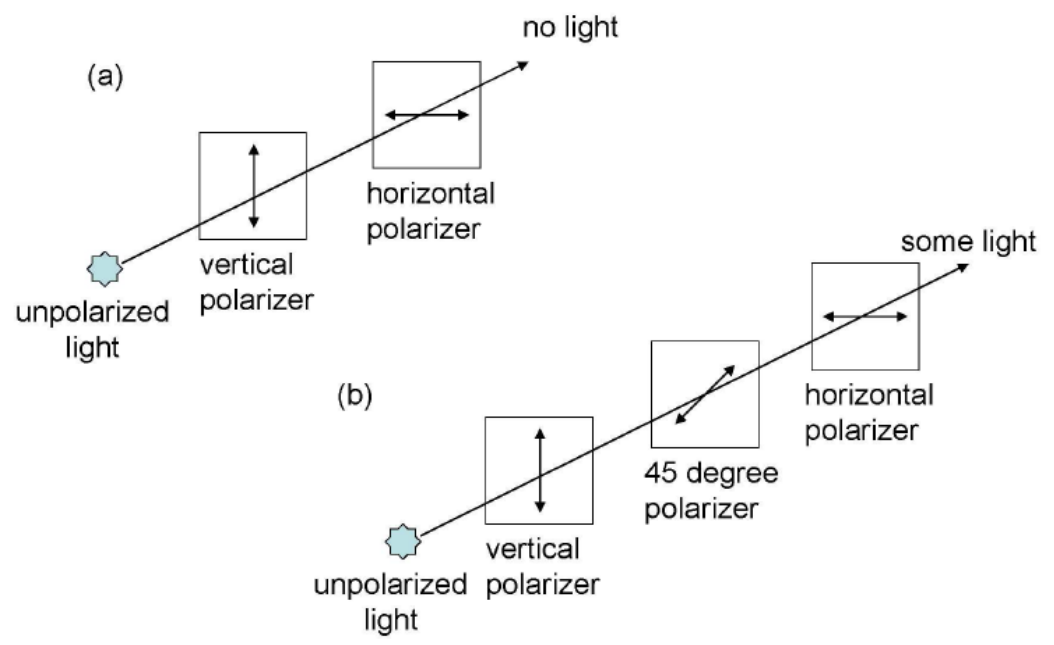

Figure 6.7 Analogy between spin states and photon polarization states. (a) Vertical light cannot directly pass through a horizontal polarizer. (b) If a 45 degree polarizer is inserted in front of the horizontal polarizer, some incoming vertical light could pass through both polarizers.

While working through the QuILT, students are asked a guided sequence of questions to help them distinguish between superposition and mixture. The QuILT presents a common incorrect point of view on the issue dealing with superposition and mixture. For example, students are asked to explain why they agree or disagree with the following statement:

- There is no difference between silver atoms in a "pure" state given by $\frac{1}{\sqrt{2}}\left(|\uparrow\rangle_{z}+|\downarrow\rangle_{z}\right)$ and an unpolarized mixture in which half of the atoms are in the $|\uparrow\rangle_{z}$ state and half are in the $|\downarrow\rangle_{z}$ state. If we had sent atoms in the superposition state $\frac{1}{\sqrt{2}}\left(|\uparrow\rangle_{z}+|\downarrow\rangle_{z}\right)$ through the SGZ, half of them would have registered in the "up" detector and half of them would have been 
collected in the lower channel. The outcome will be exactly the same if we had sent a 50/50 mixture of $|\uparrow\rangle_{z}$ and $|\downarrow\rangle_{z}$ through the SGZ. So there is no way to distinguish a mixture from a superposition.

Then, the students are given an opportunity to check their predictions using computer simulations and reconcile the differences using more guidance and support as needed. Further questions such as the following are also given to students to help them understand the difference between a pure state and a mixture by reinforcing the analogy between the spin states of electrons and the polarization states of photons:

- Remember the analogy between spin states and polarized photons. Suppose you have a beam of pure polarized photons with 45 degrees polarization and another beam of unpolarized mixture with half of the photons vertically polarized and half horizontally polarized. Will a vertical or horizontal polarizer tell you which beam is in a pure state? What polarizer could you use to differentiate the two beams of photons?

- Based upon the analogy for distinguishing between pure polarized photons and a beam of photon mixture, what kind of SGA could you use to differentiate the two beams of atoms in the previous question (which is related to distinguishing a superposition of states from a mixture)? Draw a sketch below to explain your choice. Do not forget to put the detectors in the correct positions.

The guidance provided to students is decreased as students make progress through the QuILT. In the later part of the QuILT, students are given open-ended questions such as the following:

The following questions relate to the simulation "unknown state". Run the simulation "unknown state" first. Then answer the following questions. 
- Write down at least 3 different possible spin states of the incoming particles that will show the behavior seen in the simulation. The incoming particles do not necessarily have identical spin states (can be a mixture). Explain your reasoning for your choices.

- Choose two of the different possible spin states you predicted for the simulation you saw. Now come up with some simulations using SGAs that would distinguish between the two possible spin states. You can choose one or more SGAs to find out which of the two spin states it is. Share your set-up with others in your class.

\subsection{PRELIMINARY EVALUATION}

We conducted preliminary evaluations of the SGE QuILT in two junior-senior level classes, the first with 22 and the second with 13 undergraduate students. The two classes were taught by different instructors. In both classes, students first received traditional instruction about the SGE, took a pre-test, worked on the tutorial and then took a post-test in the following class period. The test questions are given in the Appendix of this chapter (section 6.8). In particular, the first class with 22 students was given questions (1)-(4) in the pre-test and questions (5)-(7) on the post-test. The average pre-test score for this class was 53\% and the average post-test score was 92\%.

For the second class, we designed two versions of a test (versions $\mathrm{A}$ and $\mathrm{B}$ ) to assess student learning. Version A contained questions (1), (2), (3), (4) and (9) while version B had questions (1), (2), (5), (6), and (7) (see the section 6.8 for a description of all the questions). Students in the second class were randomly administered either version A or version B of the test as the pre-test after the traditional instruction. Then, each student was administered the version of the test he/she had not taken as the post-test after working on the QuILT. In particular, 8 students 
in that class were administered version A as pre-test (and version B as the post-test) whereas the other 8 students were given version $\mathrm{B}$ as the pre-test (and version $\mathrm{A}$ as the post-test). The average pre-test score for this second class was $37 \%$ and the average post-test score was $84 \%$. The average pre-test and post-test performance on each question combining the two groups of students is given in Table 6.1. Except for Question (1), on which students performed reasonably well even on the pre-test (after traditional instruction), students' performance improved on all the other questions after working on the QuILT.

Table 6.1 The pre-test and post-test scores on each question. The total number of students including both classes who answered each question is given in parenthesis.

\begin{tabular}{|c|c|c|c|c|c|c|c|c|c|}
\hline Question & 1 & 2 & 3 & 4 & 5 & 6 & 7 & 8 & 9 \\
\hline Pre-test Score(\%) & $80 \%$ & $39 \%$ & $34 \%$ & $47 \%$ & $60 \%$ & $0 \%$ & $0 \%$ & $30 \%$ & $31 \%$ \\
(Number of students) & $(35)$ & $(35)$ & $(30)$ & $(30)$ & $(5)$ & $(5)$ & $(5)$ & $(5)$ & $(8)$ \\
\hline Post-test Score(\%) & $81 \%$ & $77 \%$ & $80 \%$ & $80 \%$ & $94 \%$ & $92 \%$ & $92 \%$ & $100 \%$ & $70 \%$ \\
(Number of students) & $(13)$ & $(13)$ & $(5)$ & $(5)$ & $(30)$ & $(30)$ & $(30)$ & $(8)$ & $(5)$ \\
\hline
\end{tabular}

In Table 6.1, the improved performance on question (2) (in which students were asked about the pattern on the screen when neutral silver atoms in the spin state $|\uparrow\rangle_{z}$ were sent through a SGX-) after the QuILT suggests that students were much more likely to be able to predict the type of pattern that should form on the screen when particles in a particular spin-state pass through a SGA with a particular field gradient. Individual discussions with some students suggest that after the QuILT students had a reasonably good understanding of how to choose a good basis to analyze the spin state of a particle passing through a SGA with a particular field gradient. Some of them were not only able to write the initial spin state in an appropriate basis, they were also able to differentiate between the spin states which are vectors in the Hilbert space and the direction of the magnetic field gradient in the physical space because these are vectors in 
different spaces. In particular, during the discussions, some students correctly noted explicitly that the eigenstates of the z-component of spin are orthogonal to each other but not orthogonal to the magnetic field gradient in the z-direction in physical space. In question (3), many students realized after the QuILT that the given superposition of the eigenstates of the z-component of spin is actually an eigenstate of the x-component of spin. Student performance after the QuILT on question (7) (in which the incoming state was a general state) further suggests that they had a better understanding of how to choose a convenient basis to analyze the output of a SGA than before the QuILT. Students also performed reasonably well after the QuILT on questions for which the particle went through several SGAs in tandem (e.g., questions (4) and (6)). The improved performance on questions (5) and (9) (in which question (9) was open-ended) suggest that students had a better understanding of how a superposition of spin states and a mixture can be differentiated using SGAs. Furthermore, the improvement in the open-ended question about the preparation of a particular spin state starting from another spin-state using a SGA in question (8) is encouraging.

In addition to the pre-test and post-test, students who had used the SGE QuILT were asked the following two questions after five months in the second semester junior-senior level undergraduate quantum mechanics course. The goal was to investigate whether students can distinguish the two situations, one of which involves a superposition and another a mixture when the magnetic field gradient was explicitly provided (this question is somewhat different from question (8) on the post-test given to students five months ago in which students had to come up with their own arrangement of the SGAs):

- (a) Suppose a beam consists of silver atoms in the state $\frac{1}{\sqrt{2}}\left(|\uparrow\rangle_{z}+|\downarrow\rangle_{z}\right)$. The beam passes through a Stern Gerlach apparatus (SGA) with the magnetic field gradient in the x-direction. 
What is the minimum number of detector(s) needed to detect all the silver atoms passing through the SGA? Draw a diagram and explain your reasoning.

- (b) Suppose a beam consists of an unpolarized mixture of silver atoms in which half of the silver atoms are in state $|\uparrow\rangle_{z}$ and half are in state $|\downarrow\rangle_{z}$. The beam passes through a SGA with the magnetic field gradient in the $x$-direction. What is the minimum number of detector(s) needed to detect all the silver atoms passing through the SGA? Draw a diagram and explain your reasoning.

Eight out of nine students who answered these two questions at the end of the second semester provided the correct response for both questions. It is encouraging that the students had retained these concepts a full semester after working on the QuILT. This retention is in contrast to the performance of the graduate students on Question (1) in the Appendix at the beginning of graduate instruction discussed earlier (average score 41\%).

\subsection{SUMMARY}

We have investigated students' difficulties in quantum mechanics via the SGE and used the findings as a guide to develop a SGE QuILT. The Stern-Gerlach experiment can be used to teach many aspects of quantum mechanics effectively including issues related to measurement, importance of choosing a particular basis, differentiation between Hilbert space and real space, and the difference between a pure linear superposition of states vs. a mixture. Preliminary evaluation suggests that the QuILT is effective in improving students' understanding of concepts related to SGE. 


\subsection{APPENDIX: THE PRE-/POST-TEST QUESTIONS}

Note: Some of the questions below (or questions similar to them) were also used during the investigation of students' difficulties at various stages of the development of the QuILT.

The following information is provided in the pre-/post-test.

Figure 6.8 shows the pictorial representations used for a Stern-Gerlach apparatus (SGA). If an

atom with state $|\uparrow\rangle_{z}$ (or $|\downarrow\rangle_{z}$ ) passes through a Stern-Gerlach apparatus with the field gradient in the negative $z$-direction (SGZ-), it will be deflected in the $+z$ (or $-z$ ) direction. If an atom with state $|\uparrow\rangle_{z}$ (or $|\downarrow\rangle_{z}$ ) passes through a Stern-Gerlach apparatus with the field gradient in the positive $z$-direction (SGZ+), it will be deflected in the $-z($ or $+z)$ direction. Similarly, if an atom with state $|\uparrow\rangle_{x}$ passes through SGX- (or SGX+), it will be deflected in the $+x$ (or $-x$ ) direction. The figures below show examples of deflections through the SGX and SGZ in the plane of the paper. However, note that the deflection through a SGX will be in a plane perpendicular to the deflection through an SGZ. This actual three-dimensional nature should be kept in mind in answering the questions.
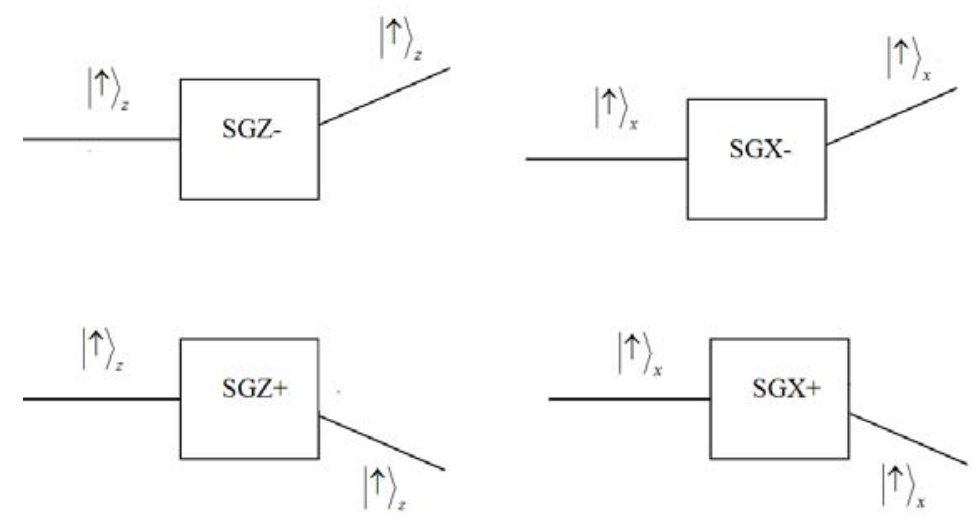

Figure 6.8 Pictorial representations used for a SGA. 
Notation: $|\uparrow\rangle_{z}$ and $|\downarrow\rangle_{z}$ represent the orthonormal eigenstates of $\hat{S}_{z}$ (the z component of the spin angular momentum). SGA is an abbreviation for a Stern-Gerlach apparatus.

(1) A beam of neutral silver atoms propagating along the y direction (into the page) in spin state $\frac{1}{\sqrt{2}}\left(|\uparrow\rangle_{z}+|\downarrow\rangle_{z}\right)$ is sent through a SGA with a vertical magnetic field gradient in the -z direction. Sketch the pattern you expect to observe on a distant phosphor screen in the $\mathrm{x}-\mathrm{z}$ plane when the atoms hit the screen. Explain your reasoning.

(2) A beam of neutral silver atoms propagating along the y direction (into the page) in spin state $|\uparrow\rangle_{z}$ is sent through a SGA with a horizontal magnetic field gradient in the $-\mathrm{x}$ direction. Sketch the pattern you expect to observe on a distant phosphor screen in the $\mathrm{x}-\mathrm{z}$ plane when the atoms hit the screen. Explain your reasoning.

(3) Chris sends silver atoms in an initial spin state $|\chi(0)\rangle=\frac{1}{\sqrt{2}}\left(|\uparrow\rangle_{z}+|\downarrow\rangle_{z}\right)$ one at a time through an SGX-. He places a "down" detector in the appropriate location as shown in Figure 6.9. What is the probability of the detector clicking when an atom exits the SGX-?

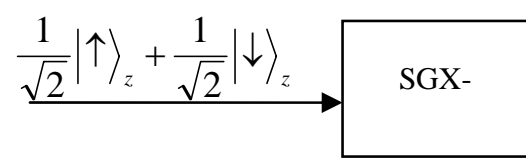

Figure 6.9 Sketch for question (3).

(4) Silver atoms in an initial spin state $|\chi(0)\rangle=|\uparrow\rangle_{z}$ pass one at a time through two SGAs with the magnetic field gradients as shown in Figure 6.10. Two suitable detectors are placed, one after the first SGA and the second at the end to detect the atoms after they 
pass through both SGAs. The atoms that do not register in the "up" detector at the end are collected for another experiment. Find the fraction of atoms that are detected in the "up" detector at the end and the normalized spin state of the atoms that are collected for another experiment.

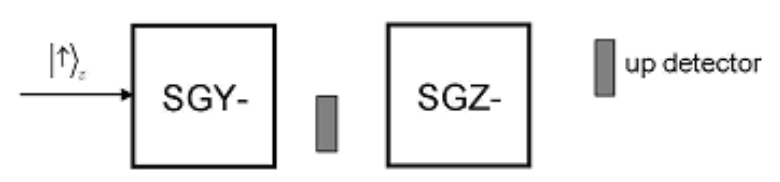

Figure 6.10 Sketch for question (4).

(5) Suppose beam A consists of silver atoms in the state $\chi=\frac{1}{\sqrt{2}}\left(|\uparrow\rangle_{z}+|\downarrow\rangle_{z}\right)$, and beam B consists of an unpolarized mixture in which half of the silver atoms are in state $|\uparrow\rangle_{z}$ and half are in state $|\downarrow\rangle_{z}$. Choose all of the following statements that are correct.

(I) Beam A will not separate after passing through SGZ-.

(II) Beam B will split into two parts after passing through SGZ-.

(III) We can distinguish between beams A and B by passing each of them through a SGX-.
A. (I) only
B. (II) only
C. (I) and (II) only
D. (II) and (III) only
E. All of the above

(6) Sally sends silver atoms in state $|\uparrow\rangle_{z}$ through three SGAs as shown in Figure 6.11. Next to each detector, write down the probability that the detector clicks. The probability for the clicking of a detector refers to the probability that a particle entering the first SGA reaches that detector. Also, after each SGA, write the spin state Sally has prepared. Explain. 


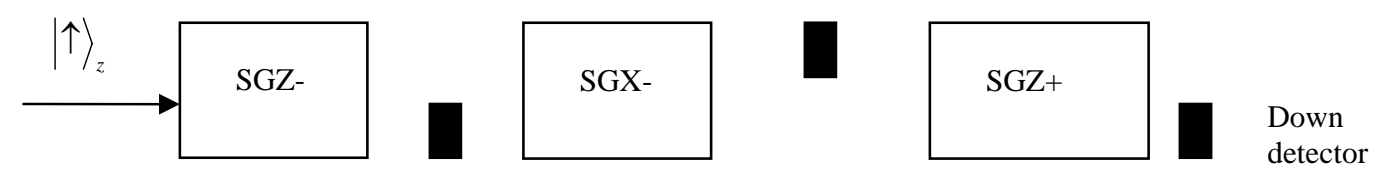

Figure 6.11 Sketch for question (6).

(7) Harry sends silver atoms all in the normalized spin state $|\chi(t=0)\rangle=a|\uparrow\rangle_{z}+b|\downarrow\rangle_{z}$ through a SGX-. He places an "up" detector as shown to block some silver atoms and collects the atoms coming out in the "lower channel" for a second experiment (see Figure 6.12). What fraction of the initial silver atoms will be available for his second experiment? What is the spin state prepared for the second experiment? Show your work.

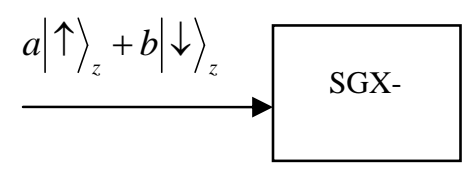

Figure 6.12 Sketch for question (7).

(8) Suppose you have a beam of atoms in the spin state $|\chi(0)\rangle=|\downarrow\rangle_{z}$ but you need to prepare the spin state $|\uparrow\rangle_{z}$ for your experiment. Could you use Stern-Gerlach Apparati and detectors to prepare the spin state $|\uparrow\rangle_{z}$ ? If yes, sketch your setup below and explain how it works. If not, explain why not.

(9) Suppose beam A consists of silver atoms in the state $\chi=\frac{1}{\sqrt{2}}\left(|\uparrow\rangle_{z}+|\downarrow\rangle_{z}\right)$, and beam B consists of an unpolarized mixture in which half of the silver atoms are in state $|\uparrow\rangle_{z}$ and half are in state $|\downarrow\rangle_{z}$. Design an experiment with Stern-Gerlach Apparati and detectors to differentiate these two beams. Sketch your experiment setup below and explain how it works. 


\subsection{CHAPTER REFERENCE}

Singh, C. (2001). "Student understanding of quantum mechanics", Am. J. Phys., 69(8), 885-896.

McDermott, L., Shaffer, P., and the Physics Education Group, University of Washington (2002). Tutorials in Introductory Physics, Upper Saddle River, NJ: Prentice Hall.

Chi, M. (1994). “Thinking Aloud”, M. Van Someren, Y. Barnard, and J. Sandberg (eds.), The Think Aloud Method: A Practical Guide to Modeling Cognitive Processes. Edited by London: Academic.

Belloni, M., Christian, W. and Cox, A. (2006). Physlet Quantum Physics, Upper Saddle River, NJ: Prentice Hall. (Simulations can be downloaded at http://www.opensourcephyscis.org)

McIntyre, D. (2002). The original Spins program was written by Daniel Schroeder and Thomas Moore for the Macintosh and was ported to Java by David McIntyre of Oregon State University and used as part of the Paradigms project. Both of these version were, and remain, open source. McIntyre's version can be found at http://www.physics.orst.edu/ $\sim$ mcintyre/ph425/spins/spinsapplet.html 


\title{
7.0 IMPROVING STUDENTS' UNDERSTANDING OF ADDITION OF ANGULAR MOMENTUM
}

\begin{abstract}
7.1 ABSTRACT
We describe the difficulties advanced undergraduate and graduate students have with the addition of angular momentum. We also describe the development and implementation of research-based learning tools such as the Quantum Interactive Learning Tutorial (QuILT) to reduce these difficulties. The preliminary evaluation shows that these learning tools are effective in improving students' understanding of concepts related to the addition of angular momentum.
\end{abstract}

\subsection{BACKGROUND}

In classical mechanics, the angular momentum vector $\vec{L}$ is defined by the cross product of the position vector $\vec{r}$ and the momentum $\vec{p}$. In quantum mechanics, the components of the angular momentum operator $\hat{L}_{x}, \hat{L}_{y}$ and $\hat{L}_{z}$ do not commute with each other and therefore the different components of angular momentum are incompatible observables. In physics, the direction in which the magnetic field is applied is taken to be the $z$-direction for standardization. The square of the magnitude of the total angular momentum operator is $\hat{L}^{2}$ and the $z$-component of the 
angular momentum operator is $\hat{L}_{z}$. The eigenvalues of the operator $\hat{L}^{2}$ are $\ell(\ell+1) \hbar^{2}$ where $\hbar=h /(2 \pi)$ ( $h$ is the Planck's constant) and $\ell$ is the angular momentum quantum number (the angular momentum quantum number can be a half-integer number for spin angular momentum, discussed in the next paragraph, but cannot be half integral for the orbital angular momentum). The eigenvalues of the $z$-component of the angular momentum operator $\hat{L}_{z}$ are $m \hbar$ where $m=-\ell,-\ell+1, \ldots, \ell$. Since $\hat{L}^{2}$ and $\hat{L}_{z}$ commute, we can use the quantum numbers $\ell$ and $m$ to denote their simultaneous eigenstates as $|\ell, m\rangle$.

In addition to the orbital angular momentum $\vec{L}$, elementary particles such as electrons also carry intrinsic spin angular momentum $\vec{S}$ which is not due to the motion in physical space (Griffiths 1995). The algebra of the orbital and spin angular momenta is similar and the components of the spin angular momentum operator $\hat{S}_{x}, \hat{S}_{y}$ and $\hat{S}_{z}$ satisfy similar commutation relations to the commutation relation between the components of the orbital angular momentum $L_{x}, L_{y}$ and $L_{z}$. The eigenvalues of the square of the magnitude of the spin angular momentum operator $\hat{S}^{2}$ are $s(s+1) \hbar^{2}$ where $s$ is the spin quantum number. For a single electron, $s=1 / 2$ and the $z$-component of the spin quantum numbers are $m_{s}=1 / 2$ or $m_{s}=-1 / 2$. If we choose the eigenstates of the z-component of spin as the basis vectors, the operators $\hat{S}_{x}=\frac{\hbar}{2} \hat{\sigma}_{x}, \hat{S}_{y}=\frac{\hbar}{2} \hat{\sigma}_{y}$, and $\hat{S}_{z}=\frac{\hbar}{2} \hat{\sigma}_{z}$ can be represented by the Pauli matrices $\hat{\sigma}_{x}=\left(\begin{array}{ll}0 & 1 \\ 1 & 0\end{array}\right), \hat{\sigma}_{y}=\left(\begin{array}{cc}0 & -i \\ i & 0\end{array}\right)$ and $\hat{\sigma}_{z}=\left(\begin{array}{cc}1 & 0 \\ 0 & -1\end{array}\right)$, respectively. Since $\hat{S}^{2}$ and $\hat{S}_{z}$ commute, we can use the quantum numbers $s$ and 
$m_{s}$ to denote their simultaneous eigenstates $\left|s, m_{s}\right\rangle$. Since $s$ is fixed for a particle with a given spin, we can use the shorthand notation $\left|s, m_{s}\right\rangle=\left|m_{s}\right\rangle$.

If a quantum system contains two particles with angular momentum quantum numbers $\ell_{1}$ and $\ell_{2}$ separately, the total angular momentum quantum number of the system can range from the summation of $\ell_{1}$ and $\ell_{2}$ down to the absolute value of the difference between them, i.e., $\ell=\ell_{1}+\ell_{2}, \ell_{1}+\ell_{2}-1, \ldots,\left|\ell_{1}-\ell_{2}\right|$. The $z$-component of the total angular momentum of the system equals the sum of the z-components of the angular momentum of the individual particles, i.e., $m=m_{1}+m_{2}$. For a single particle with non-zero spin, its total angular momentum quantum number $j$ can be obtained by the addition of its orbital angular momentum quantum number $\ell$ and its spin angular momentum quantum number $s$, i.e., $j=\ell+s, \ell+s-1, \ldots,|\ell-s|$. In this paper, we will discuss students' understanding of the addition of angular momentum for a system containing two particles with non-zero spin angular momentum (or spin).

\subsection{INVESTIGATION OF STUDENTS' DIFFICULTIES}

In this section, we discuss the investigation of students' difficulties with the addition of angular momentum in quantum mechanics. The investigation of students' difficulties with the addition of angular momentum was conducted with many undergraduate and graduate students at the University of Pittsburgh (PITT) and other universities (Singh 2006, Singh 2007) by administering written tests and by conducting in-depth individual interviews with a subset of students. 


\subsubsection{Difficulty with the Dimension of the Hilbert Space}

\section{Difficulty 1: Confusion between the Hilbert space and the Physical space}

The dimension of a Hilbert space is equal to the number of linearly independent basis vectors, e.g., the number of linearly independent eigenstates of any operator that acts on the states in that space. For example, for a particle in a one dimensional (1D) infinite square well, the

infinitely many energy eigenstates $\left|\psi_{n}\right\rangle$ corresponding to the Hamiltonian operator can form a complete set of basis vectors for the infinite dimensional Hilbert space. We have found that these concepts are very difficult for the students. For example, the following multiple choice question was given to 15 graduate students to probe whether they could distinguish between the onedimensional physical space in which the particle is confined and the infinite dimensional Hilbert space where the state of the system lies.

- Choose all of the following statements that are correct for a particle interacting with a one dimensional (1-D) infinite square well.

(1) The appropriate Hilbert space for this system is one dimensional.

(2) The energy eigenstates of the system form a basis in a 1-D Hilbert space.

(3) The position eigenstates of the system form a basis in a 1-D Hilbert space.
A. none of the above
B. 1 only
C. 2 only
D. 3 only
E. all of the above

Many students were confused about the dimensions of the Hilbert space and the physical space. Only $40 \%$ of them chose the correct answer A that none of the three statements are correct. The Hilbert space for the system in which the state of the system lies is infinite dimensional while the physical space in which the particle is confined is one dimensional. However, $20 \%$ of the students selected choice $D$ incorrectly and believed that the position eigenstates form a basis 
in a 1D Hilbert space and 13\% believed that the energy eigenstates form a basis in a 1D Hilbert space. Another $13 \%$ selected choice $B$ and believed that the Hilbert space is one dimensional but they did not believe that statements (2) and (3) were correct.

\section{Difficulty 2: Incorrectly calculating the dimension of a product space by summing the dimensions of the subspaces}

The Hilbert space for a spin-1/2 particle is two dimensional. For example, the $z$ component of the spin of an electron has only two eigenstates $\left|s=1 / 2, m_{s}=1 / 2\right\rangle$ and $\left|s=1 / 2, m_{s}=-1 / 2\right\rangle \quad$ (or $\left|m_{s}=1 / 2\right\rangle$ and $\left|m_{s}=-1 / 2\right\rangle$ for short), and the Hilbert space corresponding to the spin angular momentum of the electron is two dimensional. If a system consists of two electrons, the product space corresponding to the spin degree of freedom will be four dimensional. The basis vectors of the four dimensional product space in the uncoupled representation are $\left|m_{s 1}=1 / 2\right\rangle\left|m_{s 2}=1 / 2\right\rangle,\left|m_{s 1}=1 / 2\right\rangle\left|m_{s 2}=-1 / 2\right\rangle,\left|m_{s 1}=-1 / 2\right\rangle\left|m_{s 2}=1 / 2\right\rangle$ and $\left|m_{s 1}=-1 / 2\right\rangle\left|m_{s 2}=-1 / 2\right\rangle$. We note that the dimensionality of the four dimensional product is the product of the dimensions of the Hilbert spaces of each of the spins separately.

Students in general have great difficulties with the dimension of a product space containing two or more angular momenta. When asked about the dimension $D$ of a product space consisting of two subspaces of dimensions $D_{1}$ and $D_{2}$, many students incorrectly believed that $D=D_{1}+D_{2}$ instead of $D_{1} \times D_{2}$. Discussions with individual students suggest that such a misconception often originates from the simplest example in which students learn about the product space for two spin-1/2 particles. In this case, the dimension of the product space is four, which equals $2 \times 2$ but is also $2+2$. When we asked students about the dimension of the product 
space for a system containing one spin-1/2 particle and one spin-1 particle, many of them provided the incorrect answer $5=2+3$ instead of the correct answer $6=2 \times 3$.

\subsubsection{Difficulty in identifying different basis vectors for the product space}

For a system consisting of two spin-1/2 particles, there are two intuitive ways to represent the basis vectors for the product space. Since the spin quantum numbers $s_{1}=1 / 2$ and $s_{2}=1 / 2$ are fixed, we can use the "uncoupled representation" and express the orthonormal basis vectors for the product space as $\left|s_{1}, m_{1}\right\rangle \otimes\left|s_{2}, m_{2}\right\rangle=\left|m_{1}\right\rangle\left|m_{2}\right\rangle$ as noted earlier. In this uncoupled representation, the operators related to each particle (subspace) act on their own states, e.g.,

$\hat{S}_{1 z}|1 / 2\rangle|-1 / 2\rangle=\frac{\hbar}{2}|1 / 2\rangle|-1 / 2\rangle$ and $\hat{S}_{2 z}|1 / 2\rangle|-1 / 2\rangle=-\frac{\hbar}{2}|1 / 2\rangle|-1 / 2\rangle$. On the other hand, we can use the "coupled representation" and find the total spin quantum number of the system of two particles together. The total spin quantum number $s$ for the two spin-1/2 particle system is either $1 / 2+1 / 2=1$ or $1 / 2-1 / 2=0$. When the total spin quantum number $s$ is 1 , the quantum numbers $m_{s}$ for the $z$-components of the total spin $S_{z}$ can be 1,0 or -1 . When the total spin is 0 , the $z$ component $m_{s}$ can only be 0 . So the basis vectors of the system are $\left|s=1, m_{s}=1\right\rangle,\left|s=1, m_{s}=0\right\rangle$, $\left|s=1, m_{s}=-1\right\rangle$ and $\left|s=0, m_{s}=0\right\rangle$. In the coupled representation, the product state of a two-spin system such as $\left|s=1, m_{s}=0\right\rangle$ is not a simple product of the states of each individual spin (although we can write each coupled state as a linear superposition of a complete set of uncoupled states).

Difficulty 1: Difficulty in choosing a convenient basis and representing an operator as an $N \times N$ matrix in an $N$ dimensional product space 
Students often have difficulty in figuring out when it would be convenient to choose the basis vectors for the product space in the coupled or uncoupled representations and many have difficulty in writing an operator in a matrix form in that chosen basis. For example, when students were asked to choose a basis and write down the matrix corresponding to the operator $\hat{S}_{1} \cdot \hat{S}_{2}=\hat{S}^{2}-\hat{S}_{1}^{2}-\hat{S}_{2}^{2}$ in that basis for two spin-1/2 particles, many students had difficulty in writing a complete set of basis vectors for the product space. Moreover, those who chose the uncoupled representation often had difficulty figuring out how to write $\hat{S}^{2}$ in a matrix form. Many students did not realize that the basis vectors in the coupled representation are the eigenstates of the operator $\hat{S}^{2}$ so the matrix elements of $\hat{S}_{1} \cdot \hat{S}_{2}$ can easily be calculated in the coupled representation. Some students incorrectly thought that the basis vectors in the product space are simply a collection of the basis vectors for the subspaces. For example, for the two spin-1/2 particle system, some students wrote down the basis vectors as $\left|s_{1}=1 / 2, m_{1}=1 / 2\right\rangle$, $\left|s_{1}=1 / 2, m_{1}=-1 / 2\right\rangle,\left|s_{2}=1 / 2, m_{2}=1 / 2\right\rangle$ and $\left|s_{2}=1 / 2, m_{2}=-1 / 2\right\rangle$.

\section{Difficulty 2: Incorrectly believing that if the operator matrix is diagonal in one representation, it must also be diagonal in another representation}

To evaluate students understanding of operators in coupled/uncoupled representation, the following multiple-choice question was given to 11 students.

- Suppose the Hamiltonian of a two spin-1/2 particle system is $\hat{H}=\gamma\left(\hat{S}_{1} \cdot \vec{B}_{1}+\hat{S}_{2} \cdot \vec{B}_{2}\right)$ in which the magnetic field $\vec{B}_{1}$ and $\vec{B}_{2}$ are both in the z-direction but with different magnitudes. Choose all of the following statements that are correct.

(a) The Hamiltonian is a diagonal matrix in the coupled representation $\left|S^{2}, S_{1}^{2}, S_{2}^{2}, S_{z}\right\rangle$. 
(b) The Hamiltonian is a diagonal matrix in the uncoupled representation $\left|S_{1}^{2}, S_{1 z}\right\rangle\left|S_{2}^{2}, S_{2 z}\right\rangle$.

(c) The Hamiltonian is a $2 \times 2$ matrix $\hat{H}=\frac{\not \hbar B_{1}}{2}\left(\begin{array}{cc}1 & 0 \\ 0 & -1\end{array}\right)+\frac{\hbar \hbar B_{2}}{2}\left(\begin{array}{cc}1 & 0 \\ 0 & -1\end{array}\right)$ in the uncoupled representation.

Since the basis vectors in the coupled representation $\left|S^{2}, S_{1}^{2}, S_{2}^{2}, S_{z}\right\rangle$ are not the eigenstates of the Hamiltonian $\hat{H}=\gamma\left(\hat{S}_{1} \cdot \vec{B}_{1}+\hat{S}_{2} \cdot \vec{B}_{2}\right)$, some of the off-diagonal elements of $\hat{H}$ will be non-zero. On the other hand, in the uncoupled representation, the basis vectors $\left|S_{1}^{2}, S_{1 z}\right\rangle\left|S_{2}^{2}, S_{2 z}\right\rangle$ are the eigenstates of $\hat{H}$ and orthogonal to each other, so all of the off-diagonal elements are zero when the $\hat{H}$ matrix is expressed in the uncoupled representation. While the correct answer is (2) only, half of the students chose both the options (1) and (2). Some students incorrectly believed that the Hamiltonian must be diagonal in both the coupled and uncoupled representations. In individual discussions, students were asked to write the operator $S_{1 z}+\frac{1}{2} S_{2 z}$ for two spin-1/2 particle system in the matrix form in the product space. A student incorrectly believed that $S_{1 z}+\frac{1}{2} S_{2 z}$ is diagonal in the coupled representation. When he was told that it was not diagonal in the coupled representation he claimed “ $\ldots S_{1 z}+S_{2 z}$ is a diagonal matrix in the coupled basis. How can there be any difference between that operator and the operator $S_{1 z}+\frac{1}{2} S_{2 z}$ when it is also a superposition of $S_{1 z}$ and $S_{2 z}$ ?”. The student had failed to observe that $S_{1 z}+S_{2 z}$ is a very special superposition of $S_{1 z}$ and $S_{2 z}$ which is diagonal in both the coupled and uncoupled representations. 


\subsubsection{Difficulty in constructing an operator matrix in the product space}

To calculate the diagonal and off-diagonal matrix elements of an operator in the product space, we must sandwich the operator in between the chosen basis vectors. For example, for the

operator $\hat{S}_{1 z}+\hat{S}_{2 z}$, when we use the basis vectors in the uncoupled representation, the matrix elements are $\left\langle m_{2}^{\prime}\left|\left\langle m_{1}^{\prime}\left|\hat{S}_{1 z}+\hat{S}_{2 z}\right| m_{1}\right\rangle\right| m_{2}\right\rangle$ where $m_{1}, m_{2}, m_{1}^{\prime}, m_{2}^{\prime}$ are either $1 / 2$ or $-1 / 2$. If we choose the order of the basis vectors to be $|1 / 2\rangle|1 / 2\rangle,|1 / 2\rangle|-1 / 2\rangle,|-1 / 2\rangle|1 / 2\rangle$ and $|-1 / 2\rangle|-1 / 2\rangle$, the operator matrix would be

$$
\hat{S}_{1 z}+\hat{S}_{2 z}=\left(\begin{array}{cccc}
\hbar & 0 & 0 & 0 \\
0 & 0 & 0 & 0 \\
0 & 0 & 0 & 0 \\
0 & 0 & 0 & -\hbar
\end{array}\right) \text {. }
$$

The basis vectors in the coupled representation $\left|s, m_{s}\right\rangle$ are also a good choice to express this operator in matrix form and the matrix is diagonal since $\left|s, m_{s}\right\rangle$ are the eigenstates of the $z$ component of total spin operator $\hat{S}_{z}=\hat{S}_{1 z}+\hat{S}_{2 z}$. Thus, when we construct the operator matrix by using the basis vectors for coupled representation in the order $|1,1\rangle,|1,0\rangle,|1,-1\rangle$ and $|0,0\rangle$, the operator matrix can be expressed as

$$
\hat{S}_{1 z}+\hat{S}_{2 z}=\left(\begin{array}{cccc}
\hbar & 0 & 0 & 0 \\
0 & 0 & 0 & 0 \\
0 & 0 & -\hbar & 0 \\
0 & 0 & 0 & 0
\end{array}\right) .
$$

We can also rearrange the sequence of basis as $|1,1\rangle,|1,-1\rangle,|1,0\rangle$ and $|0,0\rangle$ in order to move the non-zero matrix elements to the upper left corner. 
Difficulty 1: Mistakenly adding algebraically the operators in different Hilbert spaces to construct the operator for the product space

We find that the students have difficulty in building the operator matrices correctly in the product space. For example, when the students were asked to construct the matrix of $\hat{S}_{1 z}+\hat{S}_{2 z}$ in a suitable basis, some of them incorrectly claimed that the resulting matrix is two dimensional and they simply added up the matrices of the operators $\hat{S}_{1 z}$ and $\hat{S}_{2 z}$, i.e.,

$$
\hat{S}_{1 z}+\hat{S}_{2 z}=\frac{\hbar}{2}\left(\begin{array}{cc}
1 & 0 \\
0 & -1
\end{array}\right)_{1}+\frac{\hbar}{2}\left(\begin{array}{cc}
1 & 0 \\
0 & -1
\end{array}\right)_{2}
$$

Some of these students placed subscripts 1 and 2 in the matrices to differentiate the two spin- $1 / 2$ particles while others just merged them into a single matrix. Similar difficulties were found when students where asked to construct a matrix for the operator $\hat{S}_{1} \cdot \hat{S}_{2}$ choosing any suitable basis. Some students simply multiplied the $2 \times 2$ matrices corresponding to each of the spins and expressed the result as another $2 \times 2$ matrix. Discussions with individual students suggest that many students have difficulty in choosing appropriate basis vectors and obtaining the matrix elements of an operator in the product space.

\section{Difficulty 2: The dimension of the operator matrix depends on the basis vectors}

Several students displayed an inconsistency in interpreting the dimension of the product space depending upon the basis chosen. For example, some students believed that the matrix for

the operator $\hat{S}_{1 z}+\hat{S}_{2 z}$ is two dimensional in the uncoupled representation. However, when asked to write the same operator in the coupled representation, some of them used the basis vectors $|1,1\rangle,|1,-1\rangle,|1,0\rangle$ and $|0,0\rangle$ and constructed a $4 \times 4$ diagonal matrix with the eigenvalues of 
$\hat{S}_{1 z}+\hat{S}_{2 z}$ in the diagonal position. These students did not realize that the dimension of the product space is independent of the representation chosen and both the uncoupled and coupled representations for the two spin-1/2 particle system have four basis vectors. Discussions with individual students suggest that some of them were unclear about the fact that the dimension of the product space should always be equal to the number of linearly independent vectors in that space and it cannot depend on the choice of basis vectors.

\section{Difficulty 3: The Hamiltonian of the system must be known in order to construct a matrix for} an operator other than the Hamiltonian operator

We found that some students believed that the Hamiltonian of the system must be given in order for them to be able to find the matrix elements of other operators. Discussions with individual students suggest that this misconception originates from several facts. For example, some students believed that since the basis vectors are often selected to be the eigenstates of the Hamiltonian, these are the only basis vectors that can be used to construct the matrix for any operator. Also, students were taught how to construct the Hamiltonian matrix for a single electron spin in a uniform magnetic field (Larmor precession of spin) and later they were taught how to construct the matrix of a Hamiltonian such as $\hat{H}=\gamma\left(\hat{S}_{1} \cdot \vec{B}+\hat{S}_{2} \cdot \vec{B}\right)$ in the product space. There was discussion about choosing a convenient basis that will make the Hamiltonian or other operators diagonal. There is also emphasis throughout the course on the role of the Hamiltonian in determining the time-evolution of the system and allowed energies. Some of the students overgeneralized the importance of the Hamiltonian in other contexts and claimed that they cannot 
construct the matrix for the operator $\hat{S}_{1 z}+\frac{1}{2} \hat{S}_{2 z}$ in the product space without knowing the Hamiltonian of the system.

\subsubsection{Difficulty in finding the probabilities for measuring an observable}

We find that students often have difficulty in understanding that a particular choice of basis vectors for the product space is suitable for questions related to the probabilities of measuring different observables. For example, if the question is related to the probabilities of measuring $S_{1 z}$ or $S_{2 z}$ in a product state written in the coupled representation, it is advisable to change the basis to the uncoupled representation. The coefficients in front of the normalized basis vectors in the uncoupled representation can then be related to the probabilities of measuring $S_{1 z}$ or $S_{2 z}$. Similarly, the coupled basis may be the appropriate basis for other probabilities, e.g., the probability of measuring the magnitude of the total spin angular momentum.

We found several types of difficulties related to the probabilities of measuring different observables in the product space. One type of difficulty in realizing an appropriate choice of basis in the product space for answering questions related to probabilities of measuring a particular observable. This difficulty was partly due to the fact that students did not realize which basis vectors were eigenvectors of operators corresponding to a particular observable and why it is easy to find the probabilities of measuring an observable if the state of the system is written in terms of the eigenstates of the observable. The second type of difficulty was related to transforming from one basis to another (e.g., from coupled to uncoupled or vice versa) using the Clebsch-Gordan (C-G) coefficient table and collecting all of the coefficients of similar terms before taking the absolute square of the coefficients to find the probabilities. This latter difficulty 
in product space is similar to those found for a single spin. It can be illustrated with the following example. Suppose a single spin-1/2 state is given by the following expression after certain manipulations:

$$
\left(a\left|s=\frac{1}{2}, m_{s}=\frac{1}{2}\right\rangle+a^{\prime}\left|s=\frac{1}{2}, m_{s}=-\frac{1}{2}\right\rangle\right)+\left(b\left|s=\frac{1}{2}, m_{s}=\frac{1}{2}\right\rangle+b^{\prime}\left|s=\frac{1}{2}, m_{s}=-\frac{1}{2}\right\rangle\right),
$$

where $a, a^{\prime}, b$ and $b^{\prime}$ are constant coefficients. When we asked students to calculate the probability of obtaining $\hbar / 2$ for $S_{z}$ (corresponding to $m_{s}=1 / 2$ ), several students incorrectly

responded that it is $|a|^{2}+|b|^{2}$. However, the coefficients with the same basis vector $\left|s=1 / 2, m_{s}=1 / 2\right\rangle$ should be combined first as $(a+b)\left|s=1 / 2, m_{s}=1 / 2\right\rangle$ to yield the correct

probability for measuring $\hbar / 2$ for $S_{z}$ to be $|a+b|^{2}$. This difficulty related to finding the appropriate probability amplitude by combining the coefficients of the same basis vector got worse when dealing with a product space and using the C-G coefficients to transform from one basis to another because some students believed that the same basis vectors in different brackets related to different angular momenta.

\subsection{QUILT FOR ADDITION OF ANGULAR MOMENTUM}

\subsubsection{Dimension of Hilbert space}

The QuILT related to the addition of angular momentum has two parts: one part is related to the coupled representation and another to the uncoupled representation. At the beginning of the first part of the QuILT, students are asked about the dimension of the product space for two spin-1/2 
systems. Together with the correct answer that the dimension is $4=2 \times 2$, a distractor $4=2+2$ was also given. To help students understand that the dimension of the product space is the product of the dimensions of the subspaces, another question asks students to consider whether the basis vectors in the product space are only $\left|s_{1}, m_{s 1}\right\rangle$, only $\left|s_{2}, m_{s 2}\right\rangle$ or their direct product $\left|s_{1}, m_{\mathrm{s} 1}\right\rangle \otimes\left|s_{2}, m_{\mathrm{s} 2}\right\rangle$ and the implications for the basis vectors in concrete cases. Then, the students go through a guided approach to constructing the basis vectors in the uncoupled representation for two spin-1/2 particles (each with the $z$-component of spin quantum numbers $\pm 1 / 2$ ) , e.g., $|1 / 2\rangle_{1}|-1 / 2\rangle_{2}$ or $|\uparrow\rangle_{1}|\downarrow\rangle_{2}$ and learn about the fact that the operators $\hat{S}_{1 z}$ and $\hat{S}_{2 z}$ only act on their respective subspaces in the uncoupled representation.

After help in constructing basic understanding about the uncoupled representation, students are given the following multiple-choice question:

- Consider the following statements based upon what you have learned so far in this tutorial and choose all the statements that are correct. $|1 / 2\rangle_{1}|1 / 2\rangle_{2}$ is an eigenstate of $\hat{S}_{1 z}$ and $\hat{S}_{2 z}$ but not $\hat{S}_{1}^{2}$ or $\hat{S}_{2}^{2}$.

(b) $\quad|1 / 2\rangle_{1}|1 / 2\rangle_{2}$ is an eigenstate of $\hat{S}_{1 z}, \hat{S}_{2 z}, \hat{S}_{1}^{2}$ and $\hat{S}_{2}^{2}$.

(c) $\quad|1 / 2\rangle_{1}|1 / 2\rangle_{2}$ is an eigenstate of $\hat{S}_{1 z}, \hat{S}_{2 z}, \hat{S}_{1}$ and $\hat{S}_{2}$.

Students learn that in the uncoupled representation, the basis vectors are eigenstates of the individual spin operators $\hat{S}_{1 z}, \hat{S}_{2 z}, \hat{S}_{1}^{2}$ and $\hat{S}_{2}^{2}$. They also learn to write the basis vectors in a matrix form via guided questions as follows: 
- Which one of the following is a correct matrix representation of the basis vectors $|1 / 2\rangle_{1}|1 / 2\rangle_{2},|1 / 2\rangle_{1}|-1 / 2\rangle_{2},|-1 / 2\rangle_{1}|1 / 2\rangle_{2}$ and $|-1 / 2\rangle_{1}|-1 / 2\rangle_{2}$ for a two spin-1/2 particles system?
(a) $\left(\begin{array}{l}1 \\ 1\end{array}\right),\left(\begin{array}{l}1 \\ 0\end{array}\right),\left(\begin{array}{l}0 \\ 1\end{array}\right)$ and $\left(\begin{array}{l}0 \\ 0\end{array}\right)$
(b) $\left(\begin{array}{l}1 \\ 0 \\ 0 \\ 0\end{array}\right),\left(\begin{array}{l}0 \\ 1 \\ 0 \\ 0\end{array}\right),\left(\begin{array}{l}0 \\ 0 \\ 1 \\ 0\end{array}\right)$ and $\left(\begin{array}{l}0 \\ 0 \\ 0 \\ 1\end{array}\right)$
(c) $\left(\begin{array}{l}1 \\ 0 \\ 0 \\ 0\end{array}\right),\left(\begin{array}{l}0 \\ 0 \\ 0 \\ 1\end{array}\right),\left(\begin{array}{l}1 \\ 0 \\ 1 \\ 0\end{array}\right)$ and $\left(\begin{array}{l}0 \\ 1 \\ 0 \\ 1\end{array}\right)$
(d) $\left(\begin{array}{l}1 \\ 0 \\ 1 \\ 0\end{array}\right),\left(\begin{array}{l}1 \\ 0 \\ 0 \\ 1\end{array}\right),\left(\begin{array}{l}0 \\ 1 \\ 1 \\ 0\end{array}\right)$ and $\left(\begin{array}{l}0 \\ 1 \\ 0 \\ 1\end{array}\right)$

Students are also provided guidance in constructing various matrices in the uncoupled representation by helping them learn to calculate the individual matrix elements. For example, for $\hat{S}_{1 z}$ or $\hat{S}_{1}^{2}$ operators, students learn that $\hat{S}_{1 z}|1 / 2\rangle_{1}|-1 / 2\rangle_{2}=(\hbar / 2)|1 / 2\rangle_{1}|-1 / 2\rangle_{2}$ and $\left(\hat{S}_{1 z}+\hat{S}_{2 z}\right)|1 / 2\rangle_{1}|-1 / 2\rangle_{2}=(\hbar / 2-\hbar / 2)|1 / 2\rangle_{1}|-1 / 2\rangle_{2}=0$. Then, the students can conclude that one of the matrix elements is ${ }_{2}\left\langle-1 /\left.2\right|_{1}\left\langle 1 / 2\left|\left(\hat{S}_{1 z}+\hat{S}_{2 z}\right)\right| 1 / 2\right\rangle_{1} \mid-1 / 2\right\rangle_{2}=0$.

In order to generalize their understanding of the product space to more complicated situations, students are later asked to consider the product space of a three spin-1/2 particle system in the uncoupled basis. One question explicitly asks them to consider the dimension in this case as follows:

- What is the dimensionality of the spin space of a three spin-1/2 system?
(a) 2
(b) $2+2+2=6$
(c) $3^{2}=9$
(d) $2^{3}=8$

Here, students are given an opportunity to think about the fact that the dimension of a product space is the product of the dimensions of the subspaces. They are further asked to 
construct the eight basis vectors for the product space of three spin-1/2 systems in the uncoupled representation. Students construct a complete set of eight basis vectors and then calculate several diagonal and off-diagonal matrix elements of the operator $\hat{S}_{1 z}+\hat{S}_{2 z}+\hat{S}_{3 z}$. Then, they construct the entire $8 \times 8$ matrix for the operator $\hat{S}_{1 z}+\hat{S}_{2 z}+\hat{S}_{3 z}$ in the uncoupled representation.

\subsubsection{Constructing matrices for different operators for the product space of two spin-1/2 systems in the uncoupled representation}

In the QuILT, students are asked to calculate the following matrices in the uncoupled representation: $\hat{H}_{1}=\left(4 E_{0} / \hbar\right)\left(\hat{S}_{1} \cdot \hat{S}_{2}\right)$ and $\hat{H}_{2}=-\mu\left(\hat{S}_{1} \cdot \vec{B}+\hat{S}_{2} \cdot \vec{B}\right)$. Apart from the constants $\mu$, $\vec{B}$ and $4 E_{0} / \hbar$, students must compare the properties of the operators $\hat{S}_{1 z}+\hat{S}_{2 z}$ and $\hat{S}_{1} \cdot \hat{S}_{2}$. Students learn that since the basis vectors in the uncoupled representation are orthonormal eigenstates of $\hat{S}_{1 z}$ and $\hat{S}_{2 z}$, all the off-diagonal elements of the operator $\hat{S}_{1 z}+\hat{S}_{2 z}$ are zero. Students are also asked to consider the following conceptual multiple-choice question to review why the operator $\hat{S}_{1 z}+\hat{S}_{2 z}$ can be written as a diagonal matrix in the uncoupled representation.

- $\quad \hat{H}_{2}=\mu\left(\hat{S}_{1} \cdot \vec{B}+\hat{S}_{2} \cdot \vec{B}\right)$ can be written as a diagonal matrix in the uncoupled representation because

(a) The basis vectors are eigenstates of $\hat{H}_{2}$ because $\hat{H}_{2}$ commutes with the operators $\hat{S}_{1 z}$, $\hat{S}_{2 z}, \hat{S}_{1}^{2}$ and $\hat{S}_{2}^{2}$.

(b) $\hat{H}_{2}$ is a Hamiltonian operator which must be diagonal no matter what basis you choose.

(c) We are dealing with spin-1/2 systems. $\hat{H}_{2}$ will not be diagonal if we had two spin-1 systems. 
After learning about how to construct the matrix for the operator $\hat{S}_{1 z}+\hat{S}_{2 z}$, students learn a more complicated case $\hat{H}_{1}=\left(4 E_{0} / \hbar\right) \hat{S}_{1} \cdot \hat{S}_{2}$. They are first asked the following question to help them think about why it is more convenient to write the operator in the form $\hat{S}_{1 x} \cdot \hat{S}_{2 x}+\hat{S}_{1 y} \cdot \hat{S}_{2 y}+\hat{S}_{1 z} \cdot \hat{S}_{2 z}$ rather than $\hat{S}^{2}-\hat{S}_{1}^{2}-\hat{S}_{2}^{2}$ to calculate the matrix elements when the uncoupled basis is used.

- Consider the following conversation between Pria and Mira:

Pria: Is $\left(4 E_{0} / \hbar\right) \hat{S}_{1} \cdot \hat{S}_{2}$ or $\left(2 E_{0} / \hbar\right)\left(\hat{S}^{2}-\hat{S}_{1}^{2}-\hat{S}_{2}^{2}\right)$ the more convenient form for writing $\hat{H}_{1}$ in the matrix form in the uncoupled representation without using a table?

Mira: Since the basis vectors $\left|m_{s}\right\rangle_{1}\left|m_{s}\right\rangle_{2}$ are not the eigenstates of $\hat{H}_{1}$, we have to be careful. It is the form $\left(4 E_{0} / \hbar\right) \hat{S}_{1} \cdot \hat{S}_{2}$ that is more useful because we can write $\hat{S}_{1} \cdot \hat{S}_{2}=\hat{S}_{1 x} \cdot \hat{S}_{2 x}+\hat{S}_{1 y} \cdot \hat{S}_{2 y}+\hat{S}_{1 z} \cdot \hat{S}_{2 z}$. Then we can write the $x$ and $y$ components of spin in terms of the raising and lowering operators and we know how they act on $\left|m_{s}\right\rangle_{1}\left|m_{s}\right\rangle_{2}$.

Do you agree with Mira? Explain.

Then, the students learn to rewrite the operator using the raising and lowering operators such that $\hat{S}_{1} \cdot \hat{S}_{2}=\left(\hat{S}_{1-} \cdot \hat{S}_{2+}+\hat{S}_{1+} \cdot \hat{S}_{2-}\right) / 2+\hat{S}_{1 z} \cdot \hat{S}_{2 z}$ and they practice applying the raising and lowering operators to the basis vectors in the uncoupled representation as in the following example:

- Which one of the following is correct?

(a) $\quad \hat{S}_{1-} \hat{S}_{2+}|1 / 2\rangle_{1}|1 / 2\rangle_{2}=\left(\hat{S}_{1-}|1 / 2\rangle_{1}\right)\left(\hat{S}_{2+}|1 / 2\rangle_{2}\right)=0$

(b) $\quad \hat{S}_{1-} \hat{S}_{2+}|1 / 2\rangle_{1}|1 / 2\rangle_{2}=\left(\hat{S}_{1-}|1 / 2\rangle_{1}\right)\left(\hat{S}_{2+}|1 / 2\rangle_{2}\right)=\hbar^{2}|1 / 2\rangle_{1}|1 / 2\rangle_{2}$ 
(c) $\quad \hat{S}_{1-} \hat{S}_{2+}|1 / 2\rangle_{1}|1 / 2\rangle_{2}=\left(\hat{S}_{1-}|1 / 2\rangle_{1}\right)\left(\hat{S}_{2+}|1 / 2\rangle_{2}\right)=2 \hbar^{2}|1 / 2\rangle_{1}|1 / 2\rangle_{2}$

Students learn that calculating the matrix elements of an operator in the uncoupled basis is relatively easy after expressing the operator by a combination of $\hat{S}_{1 z}, \hat{S}_{2 z}, \hat{S}_{1 \pm}$ and $\hat{S}_{2 \pm}$, so that each spin component can act on its own subspace. Students are also asked to explain the characteristics of the operators that will be diagonal in the uncoupled representation and they are given multiple opportunities to test what they predict in concrete situations and reconcile the differences if there are any.

At the end of the first part of the QuILT, students are given the following question:

- Consider the following conversation between Andy and Caroline:

Andy: For the question about choosing a basis for two spin-1/2 systems, we do not necessarily have to choose a basis in the product space which is an eigenstate of $\hat{S}_{1 z}$ and $\hat{S}_{2 z}$.

Caroline: I disagree. We must choose a basis in the product space which is an eigenstate of $\hat{S}_{1 z}$ and $\hat{S}_{2 z}$.

Whom do you agree with? Explain.

This question is used to help students understand that the basis vectors can be chosen according to our convenience. Usually, if an operator can be put into a diagonal matrix form in a particular basis, that basis may be more convenient than others. Discussion about these questions also leads to a smooth transition to other basis vectors, e.g., the coupled representation.

\subsubsection{Introducing the coupled representation}

Students are asked to list all of the possible total spin quantum numbers $s$ for the total spin angular momentum $\vec{S}=\vec{S}_{1}+\vec{S}_{2}$ for the product space of two spin-1/2 systems. They also list the 
quantum numbers for the $z$-component of total spin $m_{s}$ when $s=1$ and 0 . Students contemplate why a complete set of coupled states denoted by quantum numbers $s$ and $m_{s}$ and written as $\left|s, m_{s}\right\rangle$ forms a set of basis vectors for the product space for a system of two spin-1/2 particles. Some questions help students learn to apply different operators such as $\hat{S}^{2}, \hat{S}_{1}^{2}, \hat{S}_{2}^{2}$ and $\hat{S}_{z}$ on the states $\left|s, m_{s}\right\rangle$. Students also learn that the basis vectors in the coupled representation are orthonormal to each other. As shown in the multiple choice question below, the QuILT also helps students contemplate the differences between the coupled and uncoupled basis vectors.

- Choose all of the following statements that are correct about the difference between the "coupled" and "uncoupled" representations of the multi-spin system.

(a) In the coupled representation, you cannot decompose the product state of a two-spin system into products of states of each individual spin.

(b) In the uncoupled representation, you can decompose the product state of a two-spin system into products of states of each individual spin.

(c) The basis vectors in the uncoupled representation are eigenstates of $\hat{S}_{1}^{2}, \hat{S}_{1 z}, \hat{S}_{2}^{2}$ and $\hat{S}_{2 z}$, whereas the basis vectors in the coupled representation are eigenstates of $\hat{S}^{2}, \hat{S}_{1}^{2}, \hat{S}_{2}^{2}$ and $\hat{S}_{z}=\hat{S}_{1 z}+\hat{S}_{2 z}$.

Through these types of questions, students learn that in the coupled representation, the basis vectors in the product space are such that the individual states of the two particles cannot be separated from each other. They also observe that the basis vectors in the coupled and uncoupled representations are not the eigenstates of the same operators. For example, the basis vectors in the coupled representation are the eigenstates of the square of the total spin operator $\hat{S}^{2}$ but the basis vectors in the uncoupled representations are not the eigenstates of this operator. 
On the other hand, the basis vectors in both the coupled and uncoupled representations are eigenstates of the operators $\hat{S}_{1}^{2}$ and $\hat{S}_{2}^{2}$.

\subsubsection{Constructing matrices for different operators for the product space of two spin-1/2 systems in the coupled representation}

In the QuILT, students are given the task of writing the same operators $\hat{H}_{1}=\gamma\left(\hat{S}_{1} \cdot \hat{S}_{2}\right)$ and $\hat{H}_{2}=\mu\left(\hat{S}_{1} \cdot \vec{B}+\hat{S}_{2} \cdot \vec{B}\right)$ in the coupled representation that they had earlier learned to write in the uncoupled representation via a guided inquiry process. They are also asked to compare the matrices in the coupled representation with those in the uncoupled representation. They learn that in the coupled representation, it is convenient to write the operator $\hat{S}_{1} \cdot \hat{S}_{2}$ in the form $\hat{S}^{2}-\hat{S}_{1}^{2}-\hat{S}_{2}^{2}$ so the matrix elements can be easily calculated. They learn that the operator $\hat{S}_{1} \cdot \hat{S}_{2}$ is diagonal in the coupled representation. There are discussions in the QuILT to help students understand why the same operator is diagonal in one basis but non-diagonal in another basis. The operator $\hat{H}_{2}=\mu\left(\hat{S}_{1} \cdot \vec{B}+\hat{S}_{2} \cdot \vec{B}\right)$ is diagonal in both the coupled and uncoupled representations since the basis vectors in the coupled representation are the eigenstates of the operator $\hat{S}_{z}=\hat{S}_{1 z}+\hat{S}_{2 z}$. Students are also asked to express the matrix for $\hat{H}_{2}$ in the block diagonal form where all the non-zero terms are confined to a smaller block rather than being spread out in the full $4 \times 4$ matrix. This process helps students understand that they can arrange the order of basis vectors as they wish. 


\subsection{PRELIMINARY EVALUATION}

We designed a pretest and a posttest to assess the issues related to the addition of angular momentum. The pretest was administered to 9 undergraduate students after traditional instruction and the posttest was administered to the same group of students after they had finished the QuILT. The questions in the pretest and the posttest were very similar but used product spaces for quantum systems with different spin. In particular, in the pretest, the system contained two spin-1/2 particles, while the system in the posttest had one spin-1/2 particle and one spin-1 particle. The pretest question was as follows:

- Two spin $1 / 2$ systems (with the spin quantum numbers $s_{1}=1 / 2$ and $s_{2}=1 / 2$ ) at fixed locations in space (only consider spin degrees of freedom) interact with each other, and with a uniform magnetic field $\vec{B}$ pointing in the $+\mathrm{z}$ direction. When the magnetic field is off, the interaction between the spins is given by the Hamiltonian

$$
\hat{H}_{1}=\left(4 E_{0} / \hbar\right) \vec{S}_{1} \cdot \vec{S}_{2}=\left(2 E_{0} / \hbar\right)\left(S^{2}-S_{1}^{2}-S_{2}^{2}\right)
$$

where $\hat{S}=\hat{S}_{1}+\hat{S}_{2}$ and $E_{0}$ is a constant. The magnetic field interacts with each spin as follows:

$$
\hat{H}_{2}=-\mu\left(\hat{S}_{1} \cdot \vec{B}+\hat{S}_{2} \cdot \vec{B}\right) .
$$

(a) Write down a complete set of basis vectors for the vector space of a system of two spin1/2 particles. Explain the labels you are using to identify your basis states.

(b) Express the Hamiltonian $H_{1}$ in the basis you have chosen. (Hint: Write it down as an $N \times N$ matrix).

(c) Express the Hamiltonian $\mathrm{H}_{2}$ in the basis you have chosen.

(d) Are both $\mathrm{H}_{1}$ and $\mathrm{H}_{2}$ diagonal matrices in the basis you chose? 
For question (a) in the pretest, $22 \%$ of the students wrote the uncoupled basis and $33 \%$ of them wrote the coupled basis vectors. Another 33\% of them expressed the coupled singlet/triplet states using the uncoupled basis vectors, e.g., $|1,1\rangle=|\uparrow \uparrow\rangle$ and $|0,0\rangle=\frac{1}{\sqrt{2}}(|\uparrow \uparrow\rangle-|\downarrow \downarrow\rangle)$. One student gave an incorrect response, e.g., $|1 / 2, \pm 1 / 2\rangle_{1}$ and $|1 / 2, \pm 1 / 2\rangle_{2}$, which indicated that he believed that the basis vectors in the product space are the same as the basis vectors in the subspaces. In the posttest, $45 \%$ of the students wrote the uncoupled basis vectors and $55 \%$ of them chose the coupled basis vectors when answering question (a) (but for the product space of a spin-1/2 and a spin-1 system).

Only two out of the nine students knew how to calculate the matrices for the operators $\mathrm{H}_{1}$ and $\mathrm{H}_{2}$ in the pretest. 22\% of the students incorrectly simply added or multiplied the matrices for the spin operators for the subspaces. The other $55 \%$ of the students had no idea about how to calculate the matrix elements of the operators for a given set of basis vectors. In the posttest, one student could not calculate the matrix elements and another student incorrectly used the states $|\uparrow \uparrow\rangle$ as the basis vectors to construct a 4X4 matrix although he wrote down the coupled representation in question (a). $77 \%$ of the students in the posttest knew that the operator matrices were six dimensional but two of them who chose the uncoupled basis in question (a) still had difficulty in constructing the matrix for the operator $\mathrm{H}_{1}$ in question (b) since they did not express $\mathrm{H}_{1}$ using the raising and lowering operator. For the five students who chose the coupled representation, all of them could correctly construct the matrices for operators $\mathrm{H}_{1}$ and $\mathrm{H}_{2}$ except for a few calculation errors.

In the pretest, two students answered question (d) correctly with proper reasoning. Another two students who chose the coupled basis in question (a) also answered question (d) 
correctly but they neither calculated the operator matrices nor provided an explanation. Altogether $77 \%$ of the students did not know whether the operator matrices should be diagonal or not in a particular basis. In the posttest, two students did not answer the question (d) and another student incorrectly believed that both $\mathrm{H}_{1}$ and $\mathrm{H}_{2}$ were diagonal matrices in the uncoupled representation. The other $66 \%$ of the students who had chosen either the coupled or uncoupled representation in question (a) correctly answered question (d).

Questions (a), (b) and (c) all counted for 3 points and question (d) counted for 1 point in both the pre and post tests so that the full score is 10 for the entire test. The average correct percentage is $39 \%$ for the pretest and $73 \%$ for the posttest. When we interviewed some students individually using a think-aloud protocol, they mentioned that they believed that the uncoupled representation was always expressed with the up and down arrows. Moreover, they confused the uncoupled basis vectors $\left|S_{1 z}\right\rangle\left|S_{2 z}\right\rangle$, which is a simplified expression of $\left|S_{1}^{2}, S_{1 z}, S_{2}^{2}, S_{2 z}\right\rangle$, for basis vectors in the coupled representation. Using the feedback we modified our QuILT and we replaced the up-down arrows such as $|\uparrow \uparrow\rangle$ with the quantum numbers $| \pm 1 / 2\rangle_{1}| \pm 1 / 2\rangle_{2}$ in order to clarify the role of the quantum numbers of the individual subspaces in the uncoupled representations so that students can generalize what they learn about the product space of two spin-1/2 systems to other product spaces readily.

\subsection{SUMMARY}

We find that students have many common difficulties related to the addition of angular momentum. For example, many students were unclear about the dimension of the product space 
and they believed that the dimension of the product space is the sum of the dimensions of the subspaces. Students also had difficulty in distinguishing between the basis vectors in the coupled and uncoupled representations and had difficulty in determining how to choose an appropriate basis for the product space to answer questions related to the measurements of different observables. While changing basis using C-G coefficients, students also had difficulty in determining how to calculate the probabilities of measuring different observables. Students struggled to construct the matrix of an operator in a convenient basis in the product space. Some students believed that the dimension of a product space in the coupled and uncoupled representations is different. In particular, some students simply added the matrices for two spin$1 / 2$ particles to construct the matrix of the operator $\hat{S}_{1 z}+\hat{S}_{2 z}$ such that the resulting matrix in the product space was still two dimensions. Some students had difficulty understanding why the operator $\hat{S}_{1 z}+\hat{S}_{2 z}$ is diagonal in the uncoupled representation but $\hat{S}_{1 z}+\frac{1}{2} \hat{S}_{2 z}$ is not. Some believed that they should be given the Hamiltonian of the system to write any operator in the matrix form in a given basis.

We developed the research-based QuILT and concept tests to improve students' understanding of the addition of angular momentum. They provide a guided approach to bridge the gap between the quantitative and conceptual issues related to addition of angular momentum and help students connect different concepts and build a knowledge structure. Both these learning tools keep students actively engaged in the learning process. Our preliminary data show that the QuILT and concept tests help students better understand concepts related to the addition of angular momentum. 


\subsection{CHAPTER REFERENCE}

Griffiths, D. (1995). Introduction to Quantum Mechanics, Upper Saddle River, NJ: Prentice Hall.

Singh, C. (2006). “Assessing and improving student understanding of quantum mechanics”, in 2006 AIP Conference, Vol. 818, pp. 69-72

Singh, C. (2007). "Student difficulties with quantum mechanics formalism", in 2007 AIP Conference, Vol. 883, pp. 185-188 


\title{
8.0 IMPROVING STUDENTS' UNDERSTANDING OF QUANTUM MECHANICS BY USING PEER INSTRUCTION TOOLS IN CLASS
}

\begin{abstract}
8.1 ABSTRACT
In this chapter, we describe the cognitive and social issues in learning quantum mechanics which are important for bridging the gap between the quantitative and conceptual aspects of quantum mechanics. We also discuss the development and implementation of the research-based concept tests as the peer instruction tools to help students build a robust knowledge structure. The preliminary evaluations show that the concept tests are effective in helping students develop a good grasp of quantum mechanics.
\end{abstract}

\subsection{BACKGROUND}

Quantum mechanics provides a coherent framework for reasoning about microscopic phenomena. When the Hamiltonian of the system is modeled appropriately to account for the essential interactions, the theory of quantum mechanics has never failed to explain observations. However, the conceptual framework of quantum mechanics is often unintuitive to our daily experience in the classical world. For example, according to the quantum theory, the position, momentum, energy and other observables are generally not well-defined for a quantum system. We can only 
predict the probability of obtaining different values based upon the wavefunction when we perform a measurement. This probabilistic interpretation of quantum mechanics is challenging for students.

Moreover, according to the widely-taught Copenhagen interpretation of quantum mechanics, the measurement of a physical observable changes the wavefunction if the initial wavefunction is not the eigenfunction of the corresponding operator. Thus, the evolution of the quantum system according to the Time Dependent Schrödinger Equation (TDSE) is separated from what happens during the measurement of an observable. Students often have difficulties with the collapse of the wavefunction upon a quantum measurement. Prior research (Singh 2007) shows that many students have common misconceptions about the collapse of the wavefunction during the measurement, e.g., many students incorrectly believe that either the wavefunction gets stuck in the eigenstate of the operator after the measurement of the observable or it goes back to the original wavefunction after a long time. From the discussion with the students, we found that many students had not internalized that the wavefunction will again evolve according to the TDSE starting from the collapsed eigenfunction after the measurement.

In quantum theory, position and momentum are not independent variables that evolve in a deterministic way but are operators in the Hilbert space in which the states of the system are vectors. For a given state of the quantum system, the probability of measuring position or momentum depends on each other since the momentum space wavefunction can be retrieved from the position space wavefunction via Fourier transform, and vice versa. The eigenstates of the position or momentum operators span the Hilbert space so that any state of the system can be expressed as a linear superposition of a complete set of position eigenstates or momentum eigenstates. The measurement of position (or momentum) collapses the wavefunction of the 
system into a position (or momentum) eigenfunction with a certain probability depending on the initial state of the system. These concepts are challenging for students.

In addition to the lack of direct exposure to microscopic phenomena described by quantum mechanics, the mathematical facility required in quantum mechanics can increase students' cognitive load. Students are desired to have a good grasp of linear algebra, differential equations and complex variables, etc. If a student is not facile in mathematics, he/she may become overwhelmed by the mathematical details and may not have the opportunity to focus on the conceptual framework of quantum mechanics. Earlier research shows that conceptual learning can be impeded by the lack of mathematical facility (Singh 2007). Similarly, misconceptions about conceptual aspects of quantum mechanics also cause mathematical errors which do not occur for the students in a linear algebra course.

Many of students' misconceptions in the introductory physics are due to an overgeneralization of everyday experiences. However, one may assume that there may be advantages in quantum mechanics since the microscopic world does not directly deal with observable phenomena in daily experience so students are unlikely to have alternative conceptions. Unfortunately, this assumption is not true. Students have many misconceptions about the quantum mechanics model itself and about exploiting this model to infer the behavior of a quantum system. Students often over-generalize their intuition from the classical world to the quantum world which can lead to incorrect inferences. 


\subsection{OVERVIEW OF CONCEPT TESTS}

As mentioned in the first chapter of the thesis, the notion of the "zone of proximal development" (ZPD) focuses on what students can do on their own vs. with the help of an instructional strategy that accounts for their prior knowledge and builds on it. The unintuitive nature of quantum mechanics and other issues discussed earlier imply that scaffolding is critical for helping students learn concepts. By carefully designed instruction, scaffolding can be used to stretch students' learning far beyond their initial knowledge. We take into account the cognitive issues and students’ prior knowledge to develop the concept tests for peer instruction to help students build intuition about quantum phenomena and reduce difficulties in learning quantum mechanics.

The concept test for quantum mechanics contains about 500 multiple-choice questions. All the concept test questions focus on the conceptual aspects of quantum mechanics. In some of the concept test questions, students are expected to have the basic knowledge about calculus and linear algebra. However, complicated mathematical skills are not involved in any of the concept test questions. When a concept test question is presented in the class, students need to first consider the question by themselves and then discuss it with their partners. Students are usually given one to two minutes to answer each concept test question. After the students have submitted

their answers using the clickers, the instructor can explain the correct answer to the students and lead further discussion according to the distribution of students' answers. To encourage students to submit the answers according to their intuition of physics, most of the credits (e.g., 80\%) are awarded to the students for trying to answer the question even if they select the wrong choice. 


\subsection{SAMPLE CONCEPT TEST QUESTIONS}

The concept test questions related to a particular concept or principle of quantum mechanics are usually developed in a sequence to explain the same concept from different perspectives. For example, we can use mathematical representation in one question and graphical representation in the next question to help students build intuition about the abstract concepts in quantum mechanics. Many concept test questions are designed in "easy-moderate-difficult" or "easydifficult-difficult” types (Reay et al. 2008) to help the students learn the concepts in a guided approach. As mentioned in Chapter 3 about bound and scattering state wavefunctions, using a concept test in class can improve students' understanding of the related concepts. In this section, I will discuss several concept test questions about the bound and scattering state wavefunctions which have been used for a junior-senior level quantum mechanics course in the 2010 fall semester. The concept test questions were presented in the lecture before the students had used the QuILT on bound and scattering state wavefunctions. All the sample questions can be found in the appendix of this chapter.

The first concept test question (CT1) is a relatively easy question which shows the students a basic model of a bound state. Most students correctly recognized that the energy level given in CT1 corresponds to a bound state for a 1D finite square well. Only 2 out of 18 students incorrectly chose the option A which represents an alternative conception that the energy eigenfunction for a given quantum system can be a bound state and a scattering state simultaneously.

The second concept test question (CT2) asked the students to review several models of potential energies and judge which model allows both bound and scattering states. The difficulty level of this question was moderate. About $72 \%$ of the students chose the correct models, i.e., the 
1D finite square potential energy well and the $1 \mathrm{D}$ delta function potential energy well. The third concept test question (CT3) also asked the students to choose the potential energies that allow both bound and scattering states. However, unlike the verbal representation of the basic models in CT2, we use graphical representation to show the new models of potential energy wells in CT3. Since the students had not directly learned about these potential energies before, they must apply their previous knowledge about bound and scattering states to these novel situations in CT3. Only 33\% of the students chose the correct answer C and 50\% of the students incorrectly believed that the option (3) also allows both bound and scattering state wavefunctions. In fact, only scattering state wavefunctions can exist in the potential energy in option (3) because the possible energy levels must be higher than the potential energy at plus and minus infinity. Students had an active discussion before they submitted the answer of CT3. When some students found their ideas were incorrect, they were in a state of disequilibrium (Piaget 1964) and eager to resolve the discrepancy between their previous knowledge and the new situation.

\subsection{PRELIMINARY EVALUATION}

We have conducted many tests and surveys to investigate the effectiveness of the concept tests in helping students learn quantum mechanics. We found that students' understanding of quantum mechanics improved after using the concept tests as their peer instruction tools. To illustrate students' improvement after the concept tests, in this chapter we show the results of a quiz about the 1D infinite square potential energy well which is designed to examine students' understanding of some basic concepts and principles in quantum mechanics. Some other test and 
survey results about the effectiveness of the concept tests are discussed in Chapter 3 and Chapter 5 of this thesis.

The quiz on the $1 \mathrm{D}$ infinite square well was administered to three classes (experimental groups) taking a junior-senior level quantum mechanics course with concept tests in 2008, 2009 and 2010. The numbers of students in these classes are 25, 13 and 20 respectively. The concept test questions used in the three experimental groups were not exactly the same because we kept refining the concept tests based on both the professors' suggestions and students' responses. We also gave the quiz to a comparison group of 18 students receiving traditional instruction about quantum mechanics without using concept tests. There is a significant difference (p value less than 0.0001) in students' performances between the experimental groups (with lectures and concept tests) and the comparison group (with only traditional lectures). We have also observed continuous improvement in the experimental groups of students through 2008 to 2010 as we refine our concept tests.

The quiz contained 7 multiple-choice questions and 3 open-ended questions, all of which tested the basic quantum mechanical concepts related to the model of a $1 \mathrm{D}$ infinite square potential energy well, e.g., possible wavefunctions allowed in an infinite square well, time evolution of the wavefunction in the well, energy or position measurement, etc. Each question is assigned one credit and the total score for this quiz is 10 . The average score for the three experimental groups in 2008, 2009 and 2010 are 5.5, 7.0 and 7.6, respectively. The average score for the comparison group students is only 1.8. The distribution of students' individual scores is shown in Figure 5.1. The horizontal axis represents the possible scores that a student can obtain and the vertical axis represents the percentage of students in each of the four groups who obtained a particular score. The comparison group and the experimental groups in 2008, 2009 
and 2010 are marked in the colors of red, blue, yellow and green respectively. For example, the green bar on the rightmost of Figure 8.1 indicates that $30 \%$ of the students in the experimental group in 2010 got the full score (10 points) in the quiz. In the comparison group with only traditional instruction, most students got scores below 3 points and only one student got 6 points as the highest score. However, in each of the experimental groups with concept tests, most students obtained scores greater than 4 points. As we refined the concept tests through 2008 to 2010, higher percentages of students obtained high scores (9 points and 10 points) in the quiz on the 1D infinite square well.

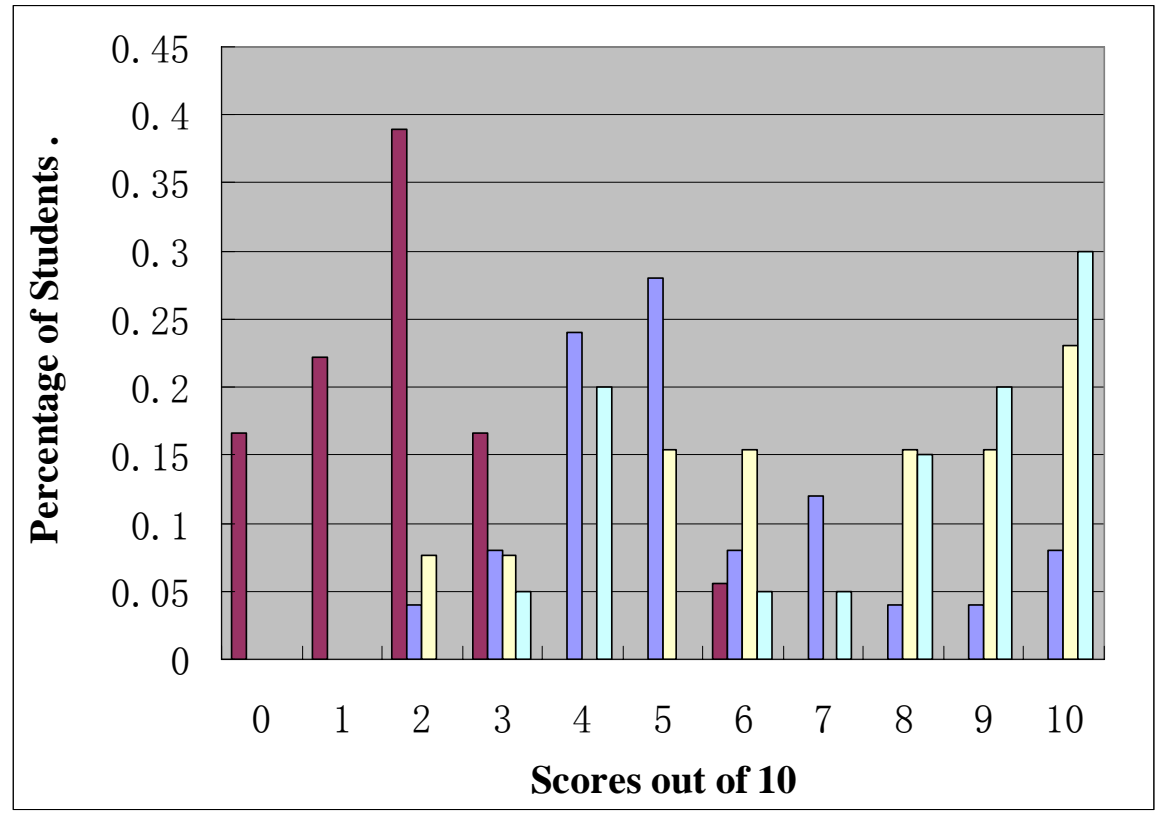

Figure 8.1 The distribution of students' individual scores on the 1D infinite square well quiz. The horizontal axis represents the possible scores on the quiz that a student can obtain and the vertical axis represents the percentage of students in each of the four groups who obtained a particular score. The comparison group and the experimental groups in 2008, 2009 and 2010 are marked in the colors of red, blue, yellow and green, respectively. 


\subsection{CONCLUSION}

We have used the concept tests as the peer instruction tools in junior-senior level quantum

mechanics courses for three years. The comparison between the classes using concept tests and the class having only traditional lectures suggests that the concept tests are effective in improving students' understanding of quantum mechanics. We also observed continuous improvement of students' performance in the conceptual surveys of quantum mechanics as we refined our concept tests through 2008 to 2010.

\subsection{APPENDIX}

\section{Concept Test Question 1}

Which one of the following statements is correct about an electron in a finite square well with a definite energy $E$ as shown in Figure 8.2.

A. The electron is in $a$ bound state between $x=0$ and $x=a$ and is in a scattering state everywhere else.

B. The electron is in a bound state.

C. The electron is in a scattering state.

D. Whether the electron is in a bound or scattering state cannot be determined without knowing the wavefunction of the electron.

E. None of the above

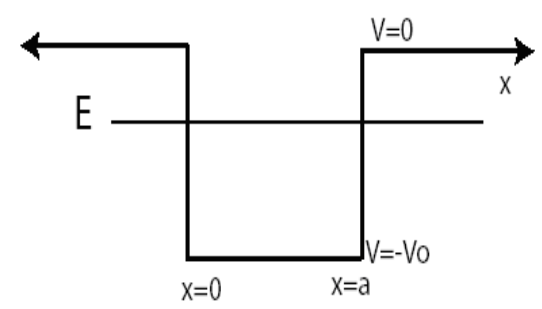

Figure 8.2 An electron with energy E interacting with a 1D finite square well. 


\section{Concept Test Question 2}

Choose all of the following $1 D$ potential energy functions that allow both bound and scattering states.

(1) Finite square well

(2) Delta function potential energy well

(3) Delta function potential energy barrier

(4) Simple harmonic oscillator potential energy
A. 1 only
B. 2 only
C. 1 and 2 only
D. 1, 2 and 3 only E. all of the above

\section{Concept Test Question 3}

Choose all of the following $1 D$ potential energies (Figure 8.3) that allow both bound and scattering states.
A. all
B. 2 only
C. 2 and 4 only
D. 3 and 4 only
E. 2, 3 and 4 only

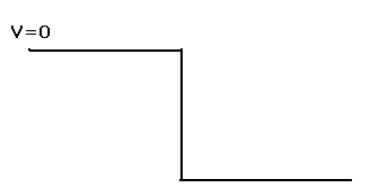

(1)

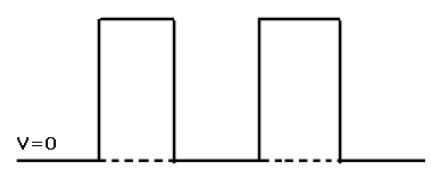

(3)

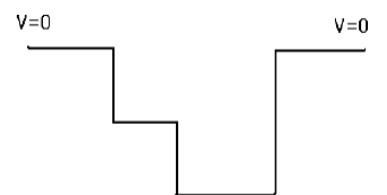

(2)

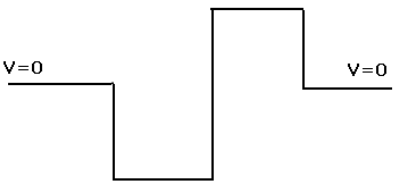

(4)

Figure 8.3 Potential energies that may allow bound states or scattering states or both. 


\subsection{CHAPTER REFERENCE}

Singh, C. (2007). "Student difficulties with quantum mechanics formalism" In 2007 Physics Education Research Conference, L. Hsu, C. Henderson, and L. McCullough, (eds.), AIP Conf. Proc. 883, pp. 185-188.

Reay, N., Li, P. and Bao, L. (2008) “Testing a new voting machine question methodology”, Am. J. Phys, 76(2), 171-178

Piaget, J. (1964). "Development and learning", R. Ripple and V. Rockcastle, (eds.), Piaget Rediscovered. New York: Cornell University Press, pp. 29. 


\subsection{SURVEYING STUDENTS' UNDERSTANDING OF QUANTUM MECHANICS}

\subsection{ABSTRACT}

Development of conceptual multiple-choice tests related to a particular physics topic is important for designing research-based learning tools to reduce the difficulties. We explore the difficulties that the advanced undergraduate and graduate students have with non-relativistic quantum mechanics of one particle in one spatial dimension. We developed a research-based conceptual multiple-choice survey that targets these issues to obtain information about the common difficulties and administered it to more than a hundred students from seven different institutions. The issues targeted in the survey include the set of possible wavefunctions, bound and scattering states, quantum measurement, expectation values, the role of the Hamiltonian, time-dependence of the wavefunction and time-dependence of the expectation value. We find that the advanced undergraduate and graduate students have many common difficulties with these concepts and that research-based tutorials and peer-instruction tools can significantly reduce these difficulties. The survey can be administered to assess the effectiveness of various intructional strategies. 


\section{$9.2 \quad$ INTRODUCTION}

Learning quantum mechanics (QM) is challenging. The QM formalism is not very intuitive and it is very different from classical mechanics that students are used to from their everyday experiences and previous courses (Singh 2001, Jolly et al. 1998, Wittmann et al. 2002, Singh et al. 2006, Singh 2008a \& 2008b). Moreover, a good understanding of the QM formalism requires a good grasp of mathematics including linear algebra, differential equations and special functions. Despite the mathematical facility required to master QM, the formalism of QM has a coherent conceptual framework. For learning to be meaningful, it is critical that the goals of a QM course, the instructional design and the assessment of learning are all aligned with each other. Since the students focus on what they are assessed on, the assessment of learning in QM should not only rely on measuring their facility with solving differential equations, it should also focus on their understanding of the conceptual framework and knowledge structure of QM. Without a coherent framework, students are unlikely to retain much after the QM course is over.

Research-based conceptual multiple choice surveys are useful tools for evaluating students’ understanding of various topics (Aubrecht \& Aubrecht 1983, Nitko 1996). The multiple choice surveys are easy to administer and grade. Their scores are objective and amenable to statistical analysis so that different instructional methods or different student populations can be readily compared. The Force Concept Inventory (FCI) is a conceptual multiple-choice test (Hestenes et al. 1992) that helped many instructors recognize that many introductory physics students were not developing a functional understanding of force concepts although they performed reasonably well on quantitative problems (often using an algorithmic approach). Other conceptual surveys have also been designed for many physics topics, e.g., electricity \& magnetism (Maloney et al. 2001). These surveys reveal that students have many common 
conceptual difficulties with different topics in classical physics. Research-based instructional strategies have been shown to improve students' conceptual understanding of some of these topics significantly (Hake 1998).

The conceptual difficulties that students have in the upper-level undergraduate courses (and even graduate students) are manifested even within the most stripped-down versions of quantum mechanics, i.e., non-relativistic quantum mechanics of one particle in one spatial dimension. We developed the Quantum Mechanics Survey (QMS), which is a 31-item multiple choice test covering various topics. The survey was developed by consulting with many QM instructors about the goals of their course, the topics their students should have definitely mastered and by iterating different versions of the open-ended and multiple-choice questions with a subset of them at various stages of the development of the survey. To investigate students' difficulties with various concepts, we administered free-response and multiple-choice questions and conducted interviews with individual students using a think-aloud protocol (Chi 1994). Individual interviews with the students during the investigation of the difficulties and the development of the survey were useful to obtain an in-depth understanding of students' thought processes.

\subsection{SURVEY DESIGN}

The QMS focuses on assessing students’ understanding of the conceptual framework of QM instead of assessing their mathematical skills. One can reason about all of the questions in the QMS conceptually and one need not necessarily perform any complicated integrals in order to answer them. Since the QMS focuses on quantum systems in one spatial dimension, the concept 
of orbital angular momentum was excluded from the survey. We also deliberately excluded the spin angular momentum and the Dirac notation from the QMS to ensure that the survey can be used after most of the first semester junior-senior level QM courses regardless of the textbook, the institution or the instructor.

We paid particular attention to the issues of reliability and validity (Aubrecht \& Aubrecht 1983, Nitko 1996) while designing the QMS. Reliability refers to the relative degree of consistency between the test scores if an individual repeats the test procedures. Validity refers to the appropriateness of interpreting the test scores. To ensure that the survey is valid, the opinions of 12 instructors about the goals of a junior-senior level QM course and the concepts their students should have definitely learned were taken into account during the development of the QMS. Apart from asking the instructors about these issues in online surveys, we discussed these issues individually with several instructors at the University of Pittsburgh (Pitt) who had taught QM at the junior-senior undergraduate level and/or at the graduate level.

The QMS includes a wide range of topics that the instructors expected their students to know such as the set of possible wavefunctions for a quantum system, the expectation value of a physical observable and its time dependence, the role of the Hamiltonian of a system, the stationary states and the non-stationary states and issues related to their time development, and quantum measurements. The quantum mechanical models in the QMS are all confined to one spatial dimension (1D), e.g., the 1D infinite/finite square well, the 1D simple harmonic oscillator (SHO) and the free particle. Before developing the questions for the QMS, we first developed a test blueprint based upon the instructors' feedback which provided a framework for deciding the desired test attributes. The specificity of the test plan helped us to determine the extent of content covered and the cognitive complexity levels of the questions. 
In developing good alternative choices for the multiple-choice questions, we took advantage of the prior investigations of advanced students' difficulties with various topics in quantum mechanics at the junior/senior level, e.g., the set of possible wavefunctions, quantum measurements, time dependence of the wavefunction and expectation values (Singh 2001, Jolly et al. 1998, Wittmann et al. 2002, Singh et al. 2006, Singh 2008a \& 2008b). The alternative choices for each question often had distractors which reflected students' common misconceptions to increase the discriminating properties of the questions. Having good distractors in the alternative choices is important so that the students do not select the correct answer for the wrong reason. To further investigate students' difficulties with some concepts before designing the multiple-choice questions for the QMS, we developed and administered to the students open-ended (or free-response) questions. The answers to the open-ended questions were summarized and categorized and helped us develop good alternative choices for the multiple-choice questions in the QMS. Statistical analysis such as distribution of choices and correlation between distractors was conducted on the multiple-choice questions as they were developed and refined.

We also interviewed individual students using a think-aloud protocol (Chi 1994) to develop a better understanding of students' reasoning process when they were answering the open-ended and multiple-choice questions. During these interviews, some previously unnoticed difficulties and misconceptions were revealed. These common difficulties were incorporated into the newer version of the written tests and ultimately into the multiple-choice questions in the survey developed. Four professors at Pitt reviewed the different versions of the QMS several times to examine its appropriateness and relevance for the upper-level undergraduate QM courses and to detect any possible ambiguity in item wording. Many professors from other 
universities have also provided valuable comments to fine tune the survey. Some of the questions were inspired by the learning tools for QM such as the concept tests and Quantum Interactive Learning Tutorials (QuILTs) we have developed (Singh 2008b). Students' feedback to these questions is also an important resource for us to improve the clarity of QMS. Since we wanted the QMS to be administered within one class period, the final version of the QMS is limited to 31 multiple-choice questions. Each question has one correct and four alternative choices.

\subsection{THE SURVEY RESULTS}

The QMS was administered to 109 students from seven universities (8 different classes were involved since both the upper-level undergraduate and graduate classes took the QMS at one institution). Among the 109 students, 15 were first-year graduate students enrolled in a full year graduate QM course and they were administered the QMS after their first-semester graduate level QM course. The others were undergraduate students who had taken at least a one-semester QM course at the junior-senior level. One of these junior-senior level classes in which students were enrolled for a full-year course used research-based learning tools such as concept tests and QuILTs (Singh 2008b). The QMS was given twice to this class, at the end of the first semester (11 students) and at the end of the second semester (9 students).

The average score on the QMS for 109 students (only includes the first score of the students who took it twice) is $37.5 \%$. The reliability coefficient $\alpha$ (Aubrecht $\&$ Aubrecht 1983, Nitko 1996) for the survey is 0.87 , which is reasonably good from the standards of test design. The item difficulty of each question (percentage of students who correctly answered each question), shown in Figure 9.1, approximately ranged between 0.2 and 0.8 . Most of the item 
difficulties (26 out of 31) were below 0.5. Figure 9.2 shows the item discrimination which represents the ability of a question to distinguish between the high and low performing students in the overall test. One measure of item discrimination is the point biserial discrimination (PBD) coefficient (Aubrecht \& Aubrecht 1983, Nitko 1996), which is the correlation between "the score on a particular question" for each student and "the total test score minus the score on that question" for each student. The PBD approximately ranged from 0.2 to 0.6 with half of the questions with PBD higher than 0.4 and two items with PBD lower than 0.2. The standards of test design (Aubrecht \& Aubrecht 1983, Nitko 1996) indicate that the QMS questions have reasonably good PBD.

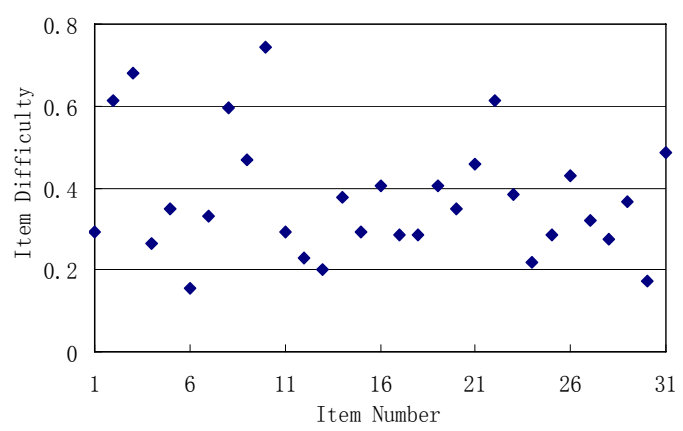

Figure 9.1 Item Difficulty (fraction correct) for each item on the test for 109 students

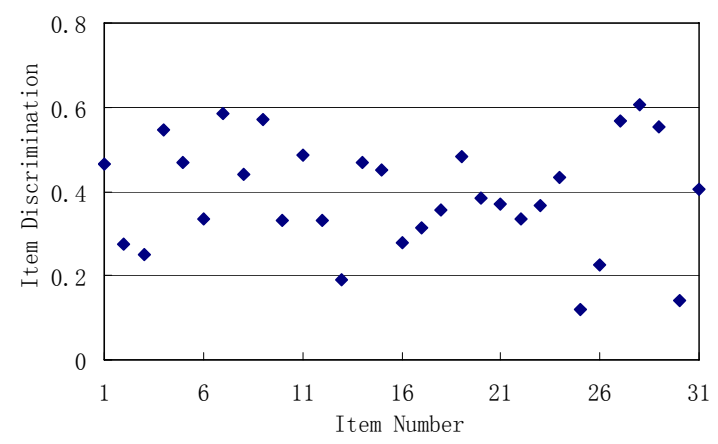

Figure 9.2 Item Discrimination (PBD coefficient) for each item on the test for 109 students

The average score for the upper-level undergraduate class that used the concept tests and the QuILTs throughout the semester was 71.8\% at the end of the first semester in which all of the 
relevant concepts were covered and 74.9\% at the end of the second semester of QM. During the second (spring) semester, these students were enrolled in the QM II course, which included identical particles and approximate methods such as the time-independent and dependent perturbation theories, variational method, scattering theory and WKB approximation. The course did not directly involve the contents in the QMS. It is encouraging that the average student performance did not deteriorate after a whole semester. In classes that did not use the researchbased learning tools, the average score was $48.4 \%$ for the graduate course (15 students) and $31.0 \%$ for the undergraduate courses. The reliability coefficients $\alpha$ were greater than 0.8 for both the class that used the research-based learning tools and the group that did not use them.

\subsection{ITEM ANALYSIS}

Table 9.1 shows one particular categorization of the questions in the QMS based upon the concepts. The table provides only one of the several possible ways to classify the questions. Our prior research shows (Lin \& Singh, 2010) that different instructors categorize a given QM question in different ways so the categorization shown in Table 9.1 is only one of them that we found convenient. The group "Other” includes questions about the uncertainty principle, the concept of degeneracy in the context of a free particle, and the Ehrenfest theorem that says that the expectation value of a physical observable obeys the classical laws (Singh 2001, Jolly et al. 1998, Wittmann et al. 2002, Singh et al. 2006, Singh 2008a \& 2008b). Below, we summarize the

common difficulties found via the QMS in the sub-category of the time dependence of expectation values. The full version of the QMS can be found in the Thesis Appendix C. 
Table 9.1 A possible categorization of the QMS questions and the number of questions belonging to each category. The same questions may address multiple categories.

\begin{tabular}{|l|c|}
\hline Concepts & Number \\
\hline Possible Wavefunctions & 5 \\
Bound/Scattering States & 5 \\
\hline Measurement & 9 \\
\hline Expectation Values & 3 \\
Time Dependence of expectation values & 4 \\
\hline Stationary vs. Non-Stationary States & 8 \\
\hline Role of the Hamiltonian & 3 \\
\hline Time Dependence of Wavefunction & 7 \\
\hline Other & 3 \\
\hline
\end{tabular}

Table 9.2 shows the percentages of students selecting the choices A-E on two problems related to the time dependence of expectation values. The correct responses are in bold italicized font. X refers to the percentage of students who did not attempt that question (left that question blank).

Table 9.2 Students' responses for the two questions about the time dependence of expectation values

\begin{tabular}{|c|c|c|c|c|c|c|}
\hline & $A$ & $B$ & $C$ & $D$ & $E$ & $X$ \\
\hline Q2 & $8 \%$ & $7 \%$ & $5 \%$ & $17 \%$ & $63 \%$ & $0 \%$ \\
\hline Q23 & $8 \%$ & $9 \%$ & $14 \%$ & $\mathbf{4 1 \%}$ & $18 \%$ & $10 \%$ \\
\hline
\end{tabular}

Questions (2) and (23) ask students about the time dependence of the expectation values of different physical observables in a stationary or a non-stationary state, respectively. The questions are as follows:

- Q2. Suppose that at time $t=0$, System I (1D infinite square well) is in the first excited state.

Choose all of the following expectation value(s) that depend on time. 

(1) $<x>$
(2) $<p>$
(3) $<H>$
A. 1 only
B. 2 only
C. 3 only
D. 1 and 2 only
E. None of the above

- Q23. Suppose at time $t=0$, System III $(1 D$ SHO $)$ is in the state $\left(\psi_{1}+\psi_{2}\right) / \sqrt{2}$. Choose all of the following expectation values that depend on time.
(1) $<x>$
(2) $<p>$
(3) $<H>$
A. 1 only
B. 2 only
C. 3 only
D. 1 and 2 only
E. All of the above

In Question (2), the initial state is an energy eigenstate, so the expectation value of any time-independent operator is time-independent. The most common mistake in Question (2) was that the students believed that the expectation values of position and momentum depend on time in a stationary state. The initial state in Question (23) is a linear superposition of the stationary states $\left(\psi_{1}+\psi_{2}\right) / \sqrt{2}$. The expectation value of energy is still time independent because the probability of obtaining energies $E_{1}$ or $E_{2}$ is always $50 \%$. But the expectation values of position and momentum depend on time. Students need not evaluate the integrals to determine the correct response. Instead, if they realize that for a non-stationary state, the probability density changes with time, they can conclude that the expectation value of position and momentum must change with time. Another way to reason is to realize that the position and momentum operators do not commute with the Hamiltonian so their expectation values will depend on time in a nonstationary state. In Question (23), 18\% of the students mistakenly thought that all the expectation values (position, momentum and energy) depend on time and $14 \%$ chose option $\mathrm{C}$ (only $<\mathrm{H}>$ depends on time) which is the opposite to the correct answer D (by contrast, for Question (2) only $5 \%$ of the students believed that $<\mathrm{H}>$ depends on time but $\langle\mathrm{x}\rangle$ and $\langle$ p $>$ do not when the system was in a stationary state). 


\subsection{SUMMARY}

Identification of students' difficulties can help catalyze the design of better instruction strategies and learning tools to improve students' understanding of QM. We have developed a researchbased multiple-choice survey to assess students' conceptual understanding of QM. The survey explores students' proficiency with the formalism of QM in 1D. During the development of the survey, we obtained feedback from QM instructors at various institutions, administered freeresponse and multiple-choice questions to students and conducted individual interviews with a subset of students to elucidate the difficulties students have with the concepts. The alternative choices for the multiple-choice questions on the survey often deal with the common difficulties found in these investigations.

The 31-item QMS was administered to 109 students in advanced undergraduate and graduate QM courses in seven different institutions to get a quantitative understanding of the universal nature of the difficulties. We found that the advanced students have common difficulties about various topics including the set of possible wavefunctions, quantum measurement, expectation values, stationary vs. non-stationary states, and time dependence of wavefunctions and expectation values. We also investigated the extent to which research-based learning tools can help students learn these concepts and found that the difficulties were significantly reduced when students used concept tests and QuILTs.

The QMS can be administered to students in the upper-level undergraduate courses after instruction. It can also be used as a preliminary test for the graduate students to evaluate their background knowledge in QM before they take the graduate-level QM courses. Those developing instructional strategies to improve students' understanding of QM can also benefit from taking into account the difficulties highlighted by the QMS. 


\subsection{CHAPTER REFERENCE}

Singh, C. (2001). "Student understanding of quantum mechanics”, Am. J. Phys., 69(8), 885-896.

Jolly, P., Zollman, D., Rebello, S. and Dimitrova, A. (1998). "Visualizing motion in potential wells”, Am. J. Phys., 66(1), 57-63.

Wittmann, M., Steinberg, R. and Redish, E. (2002). "Investigating student understanding of quantum physics: Spontaneous models of conductivity”, Am. J. Phys., 70(3), 218-226.

Singh, C., Belloni, M., and Christian, W. (2006). "Improving student's understanding of quantum mechanics." Physics Today, 8(43-49).

Singh, C. (2008a). "Student understanding of quantum mechanics at the beginning of graduate instruction”, Am. J. Phys, 76(3), 277-287

Singh, C. (2008b). "Interactive learning tutorials on quantum mechanics”, Am. J. Phys, 76(4), 400-405

Aubrecht, G. and Aubrecht, J. (1983). “Constructing objective tests”, Am. J. Phys. 51(7), 613620.

Nitko, A. (1996). Educational Assessments of Students ( $2^{\text {nd }}$ ed.), Englewood Cliffs, NJ: PrenticeHall/Merrill Education

Hestenes, D., Wells, M., and Swackhamer, G. (1992). "Force concept inventory." The Physics Teacher, 30(3), 141-158.

Maloney, D., O’Kuma, T., Hieggelke, C. and Van Heuvelen, A. (2001). “Surveying students' conceptual knowledge of electricity and magnetism”, Am. J. Phys. 69(7), S12-S23.

Hake, R. (1998). "Interactive-engagement versus traditional methods: A six-thousand-student survey of mechanics test data for introductory physics courses”, Am. J. Phys, 66(1), 6474

Chi, M. (1994). “Thinking Aloud”, M. Van Someren, Y. Barnard, and J. Sandberg (eds.), The Think Aloud Method: A Practical Guide to Modeling Cognitive Processes. Edited by London: Academic.

Lin, S. Y., and Singh, C. (2010). "Categorization of quantum mechanics problems by professors and students." Euro. J. Phys. 31, 57-68. 


\subsection{CONCLUSIONS AND FUTURE CONSIDERATIONS}

In this thesis, I discussed investigations of students' common difficulties in learning quantum mechanics. Based on these investigations, we developed research-based learning tutorials (QuILTs) and peer instruction tools that strive to reduce the difficulties and help students build a good knowledge structure of quantum mechanics. We also designed the Quantum Mechanics Survey (QMS) as an assessment tool to evaluate students' conceptual understanding of various topics in quantum mechanics.

\subsection{QUANTUM INTERACTIVE LEARNING TUTORIALS (QUILTS)}

Due to the non-intuitive nature of quantum mechanics, scaffolding is particularly important for helping students build a hierarchical knowledge structure of quantum mechanics. The QuILTs provide a guided approach to improving students' understanding of the basic concepts in quantum mechanics. In order to develop students' intuition about quantum mechanics, computerbased visualization tools are employed in several QuILTs to help students take advantage of the visual representation of the quantum mechanical concepts. Preliminary assessments indicate that the QuILTs are effective in improving students' understanding of the targeted concept and principles. 
Further research is needed to evaluate students' self-monitoring and meta-cognitive skills when they learn from the QuILTs on their own as a self-study tool. This study will be particularly useful because some instructors may be unwilling to use the class time to have students work on them as a group and may simply make them available as a self-study tool. Also, future study can investigate the retention of the students' knowledge of the concepts targeted in the QuILTs after a long time period, e.g., several years. It will also be useful to explore the facility of advanced students in transferring what they learned in one context to another context in quantum mechanics. Research can also be carried out to investigate if students are able to transfer relevant learning in the quantum mechanics course to other upper-level physics courses or whether such transfer is challenging.

\subsection{CONCEPT TESTS}

We designed the concept tests as a peer instruction tool. By discussing the concept test questions with their classmates, students are likely to learn quantum mechanics better. The concept tests are easy to integrate with traditional lectures and are likely to be adapted in a widespread manner. The results of the quizzes and surveys about various topics in quantum mechanics indicate that the students who used the concept tests in class have a better conceptual understanding of quantum mechanics than those who had only traditional lectures.

Prior research (Singh 2005) has shown that the introductory physics students can benefit from peer instruction even with minimal guidance from the instructors. In this study, the students who worked with peers not only outperformed an equivalent group of students who worked alone on the same problems, but collaboration with a partner led to "co-construction" of 
knowledge in 29\% of the cases. Co-construction of knowledge occurs when neither student who engaged in the peer collaboration was able to answer the questions by him/herself, but both gave the correct answer after working with each other on a post-test given individually to each person. Similar research using the concept tests on quantum mechanics would be very helpful in highlighting whether advanced students are more likely to co-construct knowledge than the introductory students without instructors' support. It will also be useful to explore if students in the upper-level courses such as quantum mechanics are more advanced in their communication about physics with peers compared to introductory physics students.

\subsection{QUANTUM MECHANICS SURVEY (QMS)}

Valid and reliable multiple-choice tests related to quantum mechanics concepts are important for evaluating learning and designing research-based learning tools to reduce these difficulties. We developed a research-based conceptual multiple-choice survey that targets various basic topics in quantum mechanics to obtain information about students' common difficulties. The Quantum Mechanics Survey (QMS) focuses on the fundamental concepts and principles underlying the behavior of one-dimensional quantum systems. The topics covered in the QMS were determined by collecting the opinions of many instructors in different universities about the "must-learns" in their quantum mechanics courses. The QMS was administered in seven universities. We found that the students in the junior-senior level quantum mechanics courses have common difficulties about various topics including quantum measurement, stationary vs. non-stationary states, possible wavefunctions, etc. The results of the QMS also indicated that the research-based learning tools can help students better learn these concepts and significantly reduce their 
common difficulties. Future research using this assessment tool can focus on comparing students' conceptual understanding of quantum mechanics with different teaching strategies and learning tools. It will be useful to find out whether certain difficulties and misconceptions can be effectively reduced by certain types of learning tools but not reduced by other types of learning tools.

\subsection{CHAPTER REFERENCE}

Singh, C. (2005). "Impact of peer interaction on conceptual test performance." Am. J. Phys., 73(5), 446-451. 
APPENDIX A

\section{QUILT FOR QUANTUM MEASUREMENT* (CHAPTER 5)}

*The warm-up of the tutorial for quantum measurement is not included in Appendix A.

\section{Part 1 Quantum measurement without time evolution after measurement}

Note:

$\diamond \quad$ In the simulations, the $y$-axis represents $|\psi(x)|$ (the absolute value of the wavefunction) instead of $\psi(x)$.

\section{What is quantum measurement?}

No matter what the initial state of the quantum system is, when we measure an observable, the system collapses into an eigenstate of the corresponding operator. Therefore, measurement of an observable can be considered as projecting the initial state onto an eigenstate of the operator. For example, suppose we measure the energy of a particle in the initial state $|\Psi\rangle$ which is not an energy eigenstate. Let the energy eigenstates (eigenstates of the Hamiltonian) be denoted in order of increasing energy as $\left|\psi_{1}\right\rangle,\left|\psi_{2}\right\rangle,\left|\psi_{3}\right\rangle, \ldots,\left|\psi_{n}\right\rangle, \ldots$, where $n$ is a positive integer. Then, to find the probability of measuring energy $E_{n}$, we can project the initial state $|\Psi\rangle$

onto the energy eigenstate $\left|\psi_{n}\right\rangle$ as $\left\langle\psi_{n} \mid \Psi\right\rangle$ and then calculate the probability as $\left|\left\langle\psi_{n} \mid \Psi\right\rangle\right|^{2}$. 
Now answer the following questions.

(1) Write $\left\langle\psi_{n} \mid \Psi\right\rangle$ in the position representation? (Hint : Spectral decomposition of identity gives $\left.\int d x|x\rangle\langle x|=1\right)$

(2) What is the dimension /unit of $\left\langle\psi_{n} \mid \Psi\right\rangle$ ?
A. Length, e.g., nanometer ( $\mathrm{nm}$ )
B. Inverse length, e.g., $1 / \mathrm{nm}$
C. Inverse square length, e.g., $1 / \mathrm{nm}^{2}$
D. Dimensionless / Unitless

(3) What is the physical meaning of $\left|\left\langle\psi_{n} \mid \Psi\right\rangle\right|^{2}$ ?

Now let's use the idea of projecting a general state along an energy eigenstate to find the probability of measuring a particular energy for a 1-D infinite square well.

\section{1-D infinite square well}

For a particle in a 1-D infinite square well with Hamiltonian $\hat{H}=\frac{\hat{p}^{2}}{2 m}+V(x)(V(x)=0$ when $0<x<a$ and $V(x)=+\infty$ otherwise), the $n^{\text {th }}$ energy is $E_{n}=\frac{n^{2} \pi^{2} \hbar^{2}}{2 m a^{2}}(n=1,2,3, \ldots)$, and the energy eigenfunction corresponding to $E_{n}$ is $\psi_{n}(x)=\sqrt{\frac{2}{a}} \sin \left(\frac{n \pi x}{a}\right)$ when $0<x<a$ and $\psi_{n}(x)=0$ elsewhere. Answer the following questions. (Questions 1--10) 
1. Suppose the initial state of the particle is $\left|\psi_{1}\right\rangle$. If we measure the energy of the particle, what result(s) can we obtain?
A. Only $E_{1}$
B. Any of $E_{n}, \mathrm{n}=1,2,3, \ldots$
C. $\sum c_{n} E_{n}, c_{n}$ are constants and at least two of $c_{n}$ are non-zero, $n=1,2,3, \ldots$
D. Any value of energy $E$ is possible as long as $E \geq E_{1}$

2. In the previous problem (problem 1), after the measurement of energy, what state will the particle be in?
A. Definitely in the state $\left|\psi_{1}\right\rangle$
B. Any of the states $\left|\psi_{n}\right\rangle, n=1,2,3, \ldots$
C. $\sum_{n} A_{n}\left|\psi_{n}\right\rangle, A_{n}$ are constants and at least two of $A_{n}$ are non-zero, $n=1,2,3, \ldots$
D. None of the above

\section{Simulation 1}

Double click the simulation "psi1" on the left column of the program window. The initial state of the system in this simulation is $\left|\psi_{1}\right\rangle$. Next, choose " $E$ " (energy) at the lower right corner of the new window. Click the button "measure" in the lower middle part of the window. Does the shape of the absolute value of the wave function change? Is this result consistent with your answer to question 2? What is the measured energy corresponding to the wave function you have obtained?

3. Suppose the initial state of the particle is $\frac{1}{\sqrt{2}}\left(\left|\psi_{1}\right\rangle+\left|\psi_{2}\right\rangle\right)$. If we measure the energy of the particle, what result can we obtain?
A. $\left(E_{1}+E_{2}\right) / 2$
B. $E_{1}$ or $E_{2}$
C. Any of $E_{n}, \mathrm{n}=1,2,3, \ldots$
D. $\sum c_{n} E_{n}, c_{n}$ are constants and at least two of $c_{n}$ are non-zero, $n=1,2,3, \ldots$ 
4. In the previous problem (problem 3), after the measurement of energy, what state will the particle be in?
A. $\frac{1}{\sqrt{2}}\left(\left|\psi_{1}\right\rangle+\left|\psi_{2}\right\rangle\right)$.
B. Only $\left|\psi_{1}\right\rangle$ or $\left|\psi_{2}\right\rangle$
C. Any of $\left|\psi_{n}\right\rangle$ with non-zero probability, $n=1,2,3, \ldots$
D. $\sum_{n} A_{n}\left|\psi_{n}\right\rangle, A_{n}$ are constants and at least two of $A_{n}$ are non-zero, $n=1,2,3, \ldots$
E. None of the above

5. Suppose the initial state is $|\Psi\rangle=\frac{1}{\sqrt{2}}\left(\left|\psi_{1}\right\rangle+\left|\psi_{2}\right\rangle\right)$. If you measure the energy of the system, what is the probability of measuring energy $E_{n}$ in Dirac notation? For the given initial state, the probability of measuring which of the energies is non-zero? Is this result consistent with your answers to question 3 ?

\section{Simulation 2}

Choose the simulation "psi1+psi2". The initial state of the system in this simulation is $\frac{1}{\sqrt{2}}\left(\left|\psi_{1}\right\rangle+\left|\psi_{2}\right\rangle\right)$. Next, choose “ $E$ ” (energy) at the lower right corner of the window. Then click the button "measure" in the lower middle of the window. Does the shape of the absolute value of the wave function change?

Now click the button with a curved arrow (just to the left of the measure button) to reset the initial state to $\frac{1}{\sqrt{2}}\left(\left|\psi_{1}\right\rangle+\left|\psi_{2}\right\rangle\right)$. Then measure the energy again. Do you obtain the same state after this second measurement of energy as what you obtained after the first measurement of energy in the state $\frac{1}{\sqrt{2}}\left(\left|\psi_{1}\right\rangle+\left|\psi_{2}\right\rangle\right)$ ? If yes, do you expect that you may obtain a different state when you measure energy in the next trial after resetting the initial state to $\frac{1}{\sqrt{2}}\left(\left|\psi_{1}\right\rangle+\left|\psi_{2}\right\rangle\right)$ ? Is this result consistent with your answer to question 4 ? 
Since $\left|\psi_{1}\right\rangle$ and $\left|\psi_{2}\right\rangle$ are orthogonal $\left(\left\langle\psi_{1} \mid \psi_{2}\right\rangle=0\right),\left\langle\psi_{1}\left(\frac{1}{\sqrt{2}}\left|\psi_{1}+\psi_{2}\right\rangle\right)=\frac{1}{\sqrt{2}}\left\langle\psi_{1} \mid \psi_{1}\right\rangle\right.$ and the probability of measuring $E_{1}$ and the initial state collapsing into $\psi_{1}$ after the measurement of energy is $\left|\frac{1}{\sqrt{2}}\left\langle\psi_{1} \mid \psi_{1}\right\rangle\right|^{2}=\frac{1}{2}$. Similarly, the probability of measuring $E_{2}$ and collapsing the initial state into $\psi_{2}$ after the measurement of energy is $\left|\frac{1}{\sqrt{2}}\left\langle\psi_{2} \mid \psi_{1}+\psi_{2}\right\rangle\right|^{2}=\left|\frac{1}{\sqrt{2}}\left\langle\psi_{2} \mid \psi_{2}\right\rangle\right|^{2}=\frac{1}{2}$. For any other energy eigenstate $\psi_{n},\left\langle\psi_{n} \mid \psi_{1}+\psi_{2}\right\rangle=0$, so the probability is zero for those states and the system cannot collapse to any $\left|\psi_{n}\right\rangle$ other than $\left|\psi_{1}\right\rangle$ or $\left|\psi_{2}\right\rangle$ when we measure the energy for the state $\frac{1}{\sqrt{2}}\left(\left|\psi_{1}\right\rangle+\left|\psi_{2}\right\rangle\right)$.

Now use the method of projecting the general state along an energy eigenstate to answer the following questions (questions 6-8).

6. Suppose the normalized initial state of the particle is $\sum_{n} A_{n}\left|\psi_{n}\right\rangle$, where $A_{n}$ are constants and at least two of $A_{n}$ are non-zero $(n=1,2,3, \ldots)$. If we measure the energy of the particle, what result can we obtain?
A. Any of $E_{n}$ for which $A_{n} \neq 0$.
B. $A_{n} E_{n}$
C. $\sum_{n} A_{n} E_{n}$
D. $\sum_{n}\left|A_{n}\right|^{2} E_{n}$

7. In the previous problem (question 6), what is the probability of measuring energy $E_{n}$ in the state $\sum_{n} A_{n}\left|\psi_{n}\right\rangle$ ? Note that $A_{n}$ can be a complex number.
A. $A_{n}$
B. $\left|A_{n}\right|$
C. $\left(A_{n}\right)^{2}$
D. $\left|A_{n}\right|^{2}$ 
8. In problem 6 , after the measurement of energy, what normalized state will the particle be in?

A. Any one of the energy eigenstates $\left|\psi_{n}\right\rangle$ corresponding to the energy measured.

B. Any one of the states $A_{n}\left|\psi_{n}\right\rangle$.

C. $\sum_{n} A_{n}\left|\psi_{n}\right\rangle$

D. $\sum_{n}\left|A_{n}\right|^{2}\left|\psi_{n}\right\rangle$

E. None of the above

\section{Simulation 3}

Choose the simulation "psi1+psi_n”. The initial state of the system in this simulation is $\sum_{n} A_{n}\left|\psi_{n}\right\rangle$ with equal coefficient $A_{n}$ for $n \leq 9$ and $A_{n}=0$ for $n>9$. Next, choose " $E$ " (energy) at the lower right corner of the window. Then click the button "measure" in the lower middle of the window. What state do you obtain? Set back the simulation to the initial state and measure again to check whether you can get a different state. Explain what is the probability of obtaining a particular state $\left|\psi_{n}\right\rangle$.

9. The orthonormal energy eigenfunctions $\psi_{n}$ for a 1D infinite square well satisfy $\int_{-\infty}^{+\infty} \psi_{n}^{*}(x) \psi_{m}(x) d x=\delta_{m n}$, where $\delta_{m n}=1$ when $m=n$, and $\delta_{m n}=0$ otherwise. Any state $|\Psi\rangle$ can be expressed as $|\Psi\rangle=\sum_{n} A_{n}\left|\psi_{n}\right\rangle$ because $\left|\psi_{n}\right\rangle$ form a complete set of vectors for the Hilbert space in which the state of the system lies. Find $A_{n}$ in terms of $|\Psi\rangle$ and $\left|\psi_{n}\right\rangle$ first in the Dirac notation form and then in the integral form in the position representation. (The hint is on the last page of part 1, after question 25.) 
10. Suppose the wavefunction of the particle in the initial state is $\Psi(x)=A x(a-x)$ (A is a normalization constant) when $0<x<a$ and $\Psi(x)=0$ otherwise. If we measure the energy of the particle, what is the probability of obtaining $E_{n}$ ? $(n=1,2,3, \ldots)$ Use the idea of projecting the initial state along an energy eigenstate to find the probability of measuring energy $E_{n}$. Write down your answer in both the Dirac notation and integral form in the position representation You need NOT evaluate the integral but you should show suitable limits for the integral.

For a particle interacting with a simple harmonic oscillator (SHO) potential energy, the energies are $E_{n}=\left(n+\frac{1}{2}\right) \hbar \omega(n=0,1,2, \ldots)$, and the energy eigenfunctions corresponding to $E_{n}$ are $\psi_{n}(\xi)=\left(\frac{m \omega}{\pi \hbar}\right)^{1 / 4} \frac{1}{\sqrt{2^{n} n !}} H_{n}(\xi) e^{-\xi^{2} / 2}$, where $H_{n}(\xi)$ is the $n^{\text {th }}$ Hermite polynomial and $\xi \equiv \sqrt{\frac{m \omega}{\hbar}} x$ is a dimensionless variable. The first three Hermite polynomials are $H_{0}(x)=1$, $H_{1}(x)=2 x, H_{2}(x)=4 x^{2}-2$. Answer questions $11 \& 12$.

11. Suppose the wavefunction of a simple harmonic oscillator in the initial state is a Gaussian function $\psi(\xi)=A e^{-\xi^{2} / 2}$, where $A$ is a normalization constant. If we measure the energy of the simple harmonic oscillator, what energy can we obtain?
A. $E=\hbar \omega$ only
B. $E=\sum_{n=0}^{\infty}\left(n+\frac{1}{2}\right) \hbar \omega$
C. $E=\frac{1}{2} \hbar \omega$ only
D. Any of the energies $E_{n}=\left(n+\frac{1}{2}\right) \hbar \omega, n=0,1,2, \ldots$ 
12. Suppose the initial state of a simple harmonic oscillator is a Gaussian function not centered around $x=0$ (where the potential energy is minimum). The initial state can be expressed as $\psi(\xi)=A e^{-\left(\xi-\xi_{0}\right)^{2} / 2}$, where $A$ is a normalization constant and $\xi_{0} \neq 0$. If we measure the energy of the simple harmonic oscillator, what result(s) can we obtain?
A. $E=\hbar \omega$ only
B. $E=\sum_{n=0}^{\infty}\left(n+\frac{1}{2}\right) \hbar \omega$
C. Only ground state energy $E_{0}=\frac{1}{2} \hbar \omega$ since the wavefunction is still Gaussian
D. Any of the energies $E_{n}=\left(n+\frac{1}{2}\right) \hbar \omega, \mathrm{n}=0,1,2, \ldots$

No matter what the initial state is, when we measure the energy of a quantum SHO, we always measure an energy eigenvalue (allowed energy) and collapse the wavefunction into an energy eigenstate of the SHO. It is the Hamiltonian of the system that determines the energy eigenstates and allowed energies of the system. The initial state determines the possibility of collapsing into different energy eigenstates and measuring the corresponding energy when measuring the energy of the system.

\section{Measurement of the position when the initial state is an energy eigenstate,}

Consider an electron in a 1-D infinite square well with $V=0$ when $0<x<a$ and $V=+\infty$ otherwise. Answer the following questions (questions 13 -- 18).

13. Suppose the initial state of the particle is the ground state $\left|\psi_{1}\right\rangle$. If we measure the position of the particle, what possible values can we obtain? Will we obtain the same value if we perform position measurements on a large number of identically prepared systems? Explain.
A. $x=a$ only
B. $x=a / 2$ only
C. $0<x<a$
D. Any value between $-\infty$ and $+\infty$ 
14. In the previous problem (question 13), after the measurement of position, which one of the following wavefunctions will the particle be in if we find the particle at $x=x_{0}$ ?
A. $\psi(x)=\sqrt{\frac{2}{a}} \sin \left(\frac{\pi x}{a}\right)$
B. $\psi(x)=\sqrt{\frac{2}{a}} \sin \left(\frac{\pi\left(x-x_{0}\right)}{a}\right)$
C. $\psi(x)=\delta(x)$
D. $\psi(x)=\delta\left(x-x_{0}\right)$

15. Let's find the probability density of measuring the position of the particle in state $\left|\psi_{1}\right\rangle$ (questions 13\&14) using the projection method in the Dirac notation and in the position representation. First write down the wavefunction (in the position representation) of the particle in the initial state $\left|\psi_{1}\right\rangle$ (the ground state). Then, consider the wavefunction of the position eigenstate $\left|x_{0}\right\rangle$ with eigenvalue $x_{0}$. Calculate the projection $\left\langle x_{0} \mid \psi_{1}\right\rangle$ of the state $\left|\psi_{1}\right\rangle$ along the position eigenstate $\left|x_{0}\right\rangle$ in the position represenation by writing down the integral explicitly. What is the probability density $\left|\left\langle x_{0} \mid \psi_{1}\right\rangle\right|^{2}$ for finding the particle at the position $x=x_{0}$ ? Is this result consistent with Born's interpretation of the wavefunction? Explain. (Hint: The spectral decomposition of identity is $\int_{\text {all }} d x^{\prime}\left|x^{\prime}\right\rangle\left\langle x^{\prime}\right|=1$.)

16. Born's statistical interpretation of the wavefunction says that $|\psi(x, t)|^{2} d x$ gives the probability of finding the particle between $x$ and $x+d x$ at time $t$. Does your result in question 15 support this statistical interpretation? Explain.

\section{Simulation 4}

Double click the simulation “QM measurement”. Then choose the simulation “psi1”. The initial state in this simulation is $\left|\psi_{1}\right\rangle$. Next, choose " $x$ " (position) and click the button "measure" in the lower middle of the window. What is the (approximate) position of the particle measured? Set back the simulation to the initial state $\left|\psi_{1}\right\rangle$ and measure the position again. Is the particle found at the same position as your first measurement? Explain your observation. Is this result consistent with your answer to question 13 ? 
(Note that the position eigenfunction in the simulation is not a perfect delta function due to constraints in the simulation. However, the delta function is an ideal model which does not exist in the real world. For example, when an electron in a double slit experiment hits the far away screen, it leaves a spot with a finite width.)

17. Suppose the initial state of the particle is $\frac{1}{\sqrt{2}}\left(\left|\psi_{1}\right\rangle+\left|\psi_{2}\right\rangle\right)$. If we measure the position of the particle, what result can we obtain?
A. $x=a$ only
B. $x=a / 2$ only
C. $0 \leq x \leq a$
D. Any value between $-\infty$ and $+\infty$

18. In the previous problem (question 17), after the measurement of position, what state will the particle be in if we find the particle at $x=x_{0}$ ? Write down this state in Dirac notation and in position representation. What is the probability density for measuring the position $x=x_{0}$ ? (Hint: $\int_{\text {all }} d x|x\rangle\langle x|=1$. You can calculate the projection $\left\langle x_{0} \mid \Psi\right\rangle$ in the position representation by writing down the integral $\int_{\text {all }} d x\left\langle x_{0} \mid x\right\rangle\langle x \mid \Psi\rangle=\int_{\text {all }} d x \delta\left(x-x_{0}\right) \Psi(x)$.)

\section{Simulation 5}

Double click the simulation "QM measurement”. Then choose the simulation "psi1+psi2".

The initial state of the system in this simulation is $\frac{1}{\sqrt{2}}\left(\left|\psi_{1}\right\rangle+\left|\psi_{2}\right\rangle\right)$. Next, choose " $x$ " (position) and click the button "measure" in the lower middle of the window. What is the (approximate) position of the particle? Set back the simulation to the initial state $\frac{1}{\sqrt{2}}\left(\left|\psi_{1}\right\rangle+\left|\psi_{2}\right\rangle\right)$ and measure the position again. Is the particle found at the same position as your first measurement? Explain your observation. Is this result consistent with your answer to question 17? 


\section{Measurement of the position when the initial state is an energy eigenstate of the SHO,}

Consider a particle interacting with a simple harmonic oscillator potential energy well.

Answer questions 19 \& 20 .

19. A simple harmonic oscillator is in the ground state with a normalized Gaussian wave function as shown. If we measure the position of the particle, what results can we obtain? The classical turning points are $\pm a$ where $a=\sqrt{\frac{\hbar}{m \omega}}$

A. $x=0$ only

B. $x= \pm a$ only

C. Any value between $-a$ and $a$

D. Any value between $-\infty$ and $+\infty$

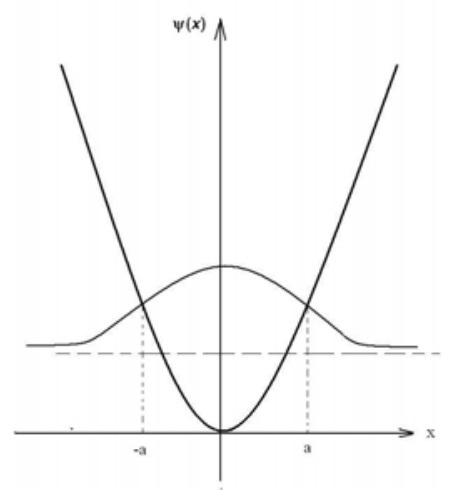

20. In the previous problem (question 19), after the measurement of position, what state will the particle be in if we find the particle at $x=x_{0}$ ? Write down this state in position representation. Use the idea of projection to write the probability density of measuring $x=x_{0}$ in Dirac notation and in the position representation when the position measurement was performed in the ground state of the SHO.

\section{Measurement of the position when the initial state is arbitrary}

Consider a particle in a 1-D infinite square well with $V=0$ when $0<x<a$ and $V=+\infty$ elsewhere. Answer the following questions (21 \& 22).

21. Suppose the wavefunction of a particle in the initial state is $\Psi(x)=A \sin ^{2}(\pi x / a)$ where $A$ is a normalization constant. If we measure the position of the particle, what is the probability density for finding the particle at $x=x_{0}$ ? Use the idea of projection to explain your answer by writing down the probability density in Dirac notation and in the position representation. 
22. In the previous problem (question 21), immediately after the measurement of position, what state will the particle be in? Write down the wavefunction of the particle in this state mathematically and also sketch it graphically in the position representation.

23. Choose all of the following statements that are correct.

(1) The shape of the position eigenfunction depends on the Hamiltonian.

(2) The shape of the energy eigenfunction depends on the Hamiltonian.

(3) No matter what kind of Hamiltonian the system has, the position eigenfunction is always a delta function in position space.
A. 1 only
B. 3 only
C. 1 and 2
D. 2 and 3
E. None of the above

24. Consider the following statement: If the initial state is $|\Psi\rangle$ for a particle in a 1-D infinite square well, $\left|\left\langle\psi_{1}|H| \Psi\right\rangle\right|^{2}$ is the probability of obtaining energy $E_{1}$ when measuring the energy of the particle. Do you agree with this statement? Explain. (Hint : Consider the unit of $\left.\left\langle\psi_{1}|H| \Psi\right\rangle.\right)$

25. For a particle in a 1-D infinite square well, suppose its initial state is $|\Psi\rangle$. What are the physical meanings of $\langle\Psi|H| \Psi\rangle$ and $\langle\Psi|x| \Psi\rangle$ ?

Hint for question 9: In position representation, $\psi(x)=\sum_{n} A_{n} \psi_{n}(x)$. Use Fourier trick.

Multiple both sides by $\psi_{m}^{*}$, integrate over all space and use orthonormality of energy eigenstates. Note that $\int_{-\infty}^{+\infty} \psi_{m}^{*}(x) \Psi(x) d x=\sum_{n} A_{n} \int_{-\infty}^{+\infty} \psi_{m}^{*} \psi_{n} d x$ and $\sum_{n} A_{n} \delta_{m n}=A_{m}$. Alternatively, in Dirac notation, $\left\langle\psi_{m} \mid \psi\right\rangle=\sum_{n} A_{n}\left\langle\psi_{m} \mid \psi_{n}\right\rangle=\sum_{n} A_{n} \delta_{m n}=A_{m}$. We can use $\int_{\text {all }}|x\rangle\langle x| d x=1$ to write $\left\langle\psi_{m} \mid \psi\right\rangle$ in position representation. 


\section{Part 2 Quantum measurement and time evolution}

For a particle in a 1-D infinite square well $(V(x)=0$ when $0<x<a$ and $V=+\infty$ elsewhere), the energies are $E_{n}=\frac{n^{2} \pi^{2} \hbar^{2}}{2 m a^{2}}(n=1,2,3, \ldots)$ and the energy eigenstate corresponding to each $E_{n}$ is $\psi_{n}(x)=\sqrt{\frac{2}{a}} \sin \left(\frac{n \pi x}{a}\right)$ when $0<x<a$ and $\psi_{n}(x)=0$ otherwise. Answer the following questions. (Question 26 34)

26. At time $t=0$, the initial state of the particle is the ground state $\psi_{1}$. If we measure the energy of the particle at time $t$, what result(s) can we obtain?
A. Only $E_{1}$
B. Only $E_{1} e^{-i E_{1} t / \hbar}$
C. Any of the energies $E_{n}, \mathrm{n}=1,2,3, \ldots$
D. Any of $E_{n} e^{-i E_{n} t / \hbar}, n=1,2,3, \ldots$
E. $\sum c_{n} E_{n}, c_{n}$ are constants and at least two of $c_{n}$ are non-zero, $n=1,2,3, \ldots$

27. In the previous problem (question 26), after the measurement of energy, what state will the particle be in?
A. The ground state $\left|\psi_{1}\right\rangle$
B. Any of the states $\left|\psi_{n}\right\rangle, n=1,2,3, \ldots$
C. $\sum_{n} A_{n}\left|\psi_{n}\right\rangle, A_{n}$ are constants and at least two of $A_{n}$ are non-zero, $n=1,2,3, \ldots$
D. None of the above

\section{Simulation 6}

- Choose the simulation "psi1". The initial state in this simulation is $\psi_{1}$. Next, click the triangular button (to start and stop the time evolution) on the lower left corner of the window. You can see a clock at the lower right corner of the window showing the time. Does the shape of the absolute value of wavefunction change with time? Why is an energy eigenstate called a "stationary state"?

- Now measure the energy around $t=2$ units. What is the state of the system after the energy 
measurement? Set back the simulation to the initial state $\left|\psi_{1}\right\rangle$ and measure the energy again around $t=3$ units. Is the result the same as your first measurement (around $t=2$ units)? Is this result consistent with your answer to question 26 ?

28. Suppose the initial state of the particle is the first excited state $\left|\psi_{2}\right\rangle$. When you measure the energy of the particle, is it possible to obtain the ground state energy $E_{1}$ ? Explain.

29. At time $t=0$, the initial state of the particle is $\frac{1}{\sqrt{2}}\left(\left|\psi_{1}\right\rangle+\left|\psi_{2}\right\rangle\right)$. If we measure the energy of the particle after time $t$, what result(s) can we obtain?

A. $\left(E_{1}+E_{2}\right) / 2$

B. $E_{1}$ or $E_{2}$

C. $\left(E_{1} e^{-i E_{1} t / \hbar}+E_{2} e^{-i E_{2} t / \hbar}\right) / 2$

D. $E_{1} e^{-i E_{1} t / \hbar}$ or $E_{2} e^{-i E_{2} t / \hbar}$

E. Any of $E_{n}, n=1,2,3, \ldots$

30. In the previous problem (question 29), right BEFORE the measurement of energy, what state will the particle be in?
A. $\frac{1}{\sqrt{2}}\left(\left|\psi_{1}\right\rangle+\left|\psi_{2}\right\rangle\right)$
B. $\left|\psi_{1}\right\rangle$ or $\left|\psi_{2}\right\rangle$
C. $\frac{1}{\sqrt{2}}\left(\left|\psi_{1}\right\rangle e^{-i E_{1} t / \hbar}+\left|\psi_{2}\right\rangle e^{-i E_{2} t / \hbar}\right)$
D. $\left|\psi_{1}\right\rangle e^{-i E_{1} t / \hbar}$ or $\left|\psi_{2}\right\rangle e^{-i E_{2} t / \hbar}$

31. In the previous problem (question 29), after the measurement of energy, what state will the particle be in?
A. $\frac{1}{\sqrt{2}}\left(\left|\psi_{1}\right\rangle+\left|\psi_{2}\right\rangle\right)$
B. Either $\left|\psi_{1}\right\rangle$ or $\left|\psi_{2}\right\rangle$
C. Any of $\left|\psi_{n}\right\rangle, n=1,2,3, \ldots$
D. $\sum_{n} A_{n}\left|\psi_{n}\right\rangle, A_{n}$ is constant and at least two of $A_{n}$ are non-zero, $n=1,2,3, \ldots$
E. None of the above 


\section{Simulation 7}

- Open the simulation "psi1+psi2". The initial state in this simulation is $\frac{1}{\sqrt{2}}\left(\left|\psi_{1}\right\rangle+\left|\psi_{2}\right\rangle\right)$. Start the time evolution. Does the shape of the absolute value of the wavefunction change with time? Is the state $\frac{1}{\sqrt{2}}\left(\left|\psi_{1}\right\rangle+\left|\psi_{2}\right\rangle\right)$ a stationary state?

- Now measure the energy around $t=2$ units. What is the state of the particle after the energy measurement? Suppose you obtain state $\left|\psi_{i}\right\rangle$ ( $i=1$ or 2 ) in the first measurement of energy. If you set back the simulation to the initial state $\frac{1}{\sqrt{2}}\left(\left|\psi_{1}\right\rangle+\left|\psi_{2}\right\rangle\right)$ and measure the energy again around the same time $t=2$ units, do you think you have the same probability of obtaining $\left|\psi_{i}\right\rangle$ as in your first measurement? Does the probability of obtaining $\left|\psi_{i}\right\rangle$ change if you re-initialize the state and measure the energy around the time $t=3$ units? (Note that you only need to write down your conclusion and explanation without measuring the energy repeatedly to estimate the probability.)

32. At time $t=0$, the initial normalized state of the particle is $\sum A_{n} \psi_{n}$, where $A_{n}$ are normalized non-zero constants. If we measure the energy of the particle at time $t$, what result can we obtain?
A. Any of $E_{n}, n=1,2,3, \ldots$
B. Any of $A_{n} E_{n}$
C. Any of $A_{n} E_{n} e^{-i E_{n} t / \hbar}$
D. $\sum_{n} A_{n} E_{n} e^{-i E_{n} t / \hbar}$
E. $\sum_{n}\left|A_{n}\right|^{2} E_{n} e^{-i E_{n} t / \hbar}$

33. In problem 32, right BEFORE the measurement of energy, what state will the particle be in?
A. $\sum_{n} A_{n}\left|\psi_{n}\right\rangle$
B. $\left|\psi_{n}\right\rangle$
C. $\sum_{n} A_{n}\left|\psi_{n}\right\rangle e^{-i E_{n} t / \hbar}$
D. $\left|\psi_{n}\right\rangle e^{-i E_{n} t / \hbar}$ 
34. In problem 33, what is the probability of measuring energy $E_{n}$ ?

(1) $A_{n} e^{-i E_{n} t / \hbar}$

(2) $\left|A_{n}\right|^{2}$

(3) $\left|A_{n} e^{-i E_{n} t / \hbar}\right|^{2}$
A. 1 only
B. 2 only
C. 3 only
D. 2 and 3 only
E. all of the above

\section{Simulation 8}

- Choose the simulation "psi1+psi_n”. The initial state in this simulation is $\sum_{n} A_{n}\left|\psi_{n}\right\rangle$. Start the time evolution. Does the shape of the absolute value of the wavefunction change with time?

- Reset the simulation to the initial state $\sum_{n} A_{n}\left|\psi_{n}\right\rangle$ and make an energy measurement at time $t=0$. Sketch the wave function you observed in the simulation. Which energy eigenstate $\left|\psi_{i}\right\rangle$ do you obtain? Which energy have you measured?

- Now reset the simulation to the initial state $\sum_{n} A_{n}\left|\psi_{n}\right\rangle$ and start the time evolution. Measure the energy around $t=2$ units. What is the state of the particle after the energy measurement? What is the energy that you measured? Write down how the state $\sum_{n} A_{n}\left|\psi_{n}\right\rangle$ evolves with time and calculate the probability of measuring energy $E_{n}$. Does the probability of measuring a particular energy $E_{n}$ and collapsing into an energy eigenstate $\left|\psi_{n}\right\rangle$ change with time? Explain. (You only need to write down your conclusion and explanation without measuring the energy repeatedly to estimate the probability.) 
For a particle interacting with a simple harmonic oscillator (SHO) potential, the allowed energies are $E_{n}=\left(n+\frac{1}{2}\right) \hbar \omega(n=0,1,2, \ldots)$, and the energy eigenstate corresponding to each $E_{n}$ is $\psi_{n}(\xi)=\left(\frac{m \omega}{\pi \hbar}\right)^{1 / 4} \frac{1}{\sqrt{2^{n} n !}} H_{n}(\xi) e^{-\xi^{2} / 2}$, where $H_{n}(\xi)$ is the $n^{\text {th }}$ Hermite polynomial and $\xi \equiv \sqrt{\frac{m \omega}{\hbar}} x$ is a dimensionless variable. The first three Hermite polynomials are $H_{0}(x)=1$, $H_{1}(x)=2 x, H_{2}(x)=4 x^{2}-2$. Answer the following questions (questions $35 \& 36$ ).

\section{Measurement of the energy of SHO at time $t>0$.}

35. At time $t=0$, suppose the initial state of a simple harmonic oscillator is a Gaussian function $\psi(x)=A e^{-\xi^{2} / 2}$, where $A$ is a positive constant. If we measure the energy of the simple harmonic oscillator at time $t$, what result can we obtain?
A. $E=\hbar \omega$ only
B. $E=\sum_{n=0}^{\infty}\left(n+\frac{1}{2}\right) \hbar \omega, n=0,1,2, \ldots$
C. $E=\frac{1}{2} \hbar \omega$ only
D. Any of the energies $E_{n}=\left(n+\frac{1}{2}\right) \hbar \omega, n=0,1,2, \ldots$

36. In the previous problem (question 35), after the measurement of energy, what state will the particle be in?
A. $\psi(x)=\sqrt{\frac{2}{a}} \sin \left(\frac{\pi x}{a}\right)$
B. $\psi(x)=\sqrt{\frac{2}{a}} \sin \left(\frac{n \pi x}{a}\right), n=1,2,3, \ldots$
C. $\psi(x)=\left(\frac{m \omega}{\pi \hbar}\right)^{1 / 4} \frac{1}{\sqrt{2^{n} n !}} H_{n}(x) e^{-x^{2} / 2}, n=0$ only
D. Any of $\psi(x)=\left(\frac{m \omega}{\pi \hbar}\right)^{1 / 4} \frac{1}{\sqrt{2^{n} n !}} H_{n}(x) e^{-x^{2} / 2}, n=1,2,3, \ldots$
E. $\psi(x)=\sum c_{n} \psi_{n}$, where $\psi_{n}(x)=\left(\frac{m \omega}{\pi \hbar}\right)^{1 / 4} \frac{1}{\sqrt{2^{n} n !}} H_{n}(x) e^{-x^{2} / 2}, n=0,1,2, \ldots$ 
Now consider a particle in a 1-D infinite square well $(V(x)=0$ when $0<x<a$ and

$V(x)=+\infty$ otherwise). Answer the following questions (question 37-40).

37. Suppose at time $t=0$ the initial state wavefunction of the particle is $\Psi(x)=A x(a-x)$ for $0<x<a$ and $\Psi(x)=0$ otherwise. If you measure the energy of the particle at time $t$, what is the probability of obtaining $E_{n}$ ? You can leave the probability as an integral. (Hint: Recall question 9 in the first part of this tutorial. You can write $\Psi(x)$ in the basis of energy eigenfunctions as $\Psi(x)=\sum_{n} A_{n} \psi_{n}$ and find the coefficients $A_{n}$ by projecting the state $|\Psi\rangle$ along the energy eigenstate $\left|\psi_{n}\right\rangle$ or by using the Fourier trick. )

38. Given the wavefunction at time $t=0$, why is it useful to write the state of a quantum system as a superposition of energy eigenstates to find the wavefunction after time $t$ ? (The answer is on the last page of the tutorial.)

\section{Measurement of postion}

39. Harry and Sally prepare the same initial wavefunction $\frac{\psi_{1}(x)+\psi_{2}(x)}{\sqrt{2}}$ which is a linear superposition of the energy eigenfunctions $\psi_{1}(x)$ and $\psi_{2}(x)$ in their labs at time $\boldsymbol{t}=\mathbf{0}$. They each make a measurement of the position of the electron after different time $\boldsymbol{t}$. The wave function at time $\boldsymbol{t}$ is $\Psi(x, t)=\frac{\psi_{1}(x) e^{-i E_{1} t / \hbar}+\psi_{2}(x) e^{-i E_{2} t / \hbar}}{\sqrt{2}}$. Harry measures the position of his electron at time $t=1$ unit and Sally measures the position of her electron at time $t=3$ units. Consider the following conversation between Harry and Sally.

Harry: The probability that I will find my electron between $x_{0}$ and $x_{0}+d x$ is not the same as the probability that you will find your electron between $x_{0}$ and $x_{0}+d x$. The probability is determined by the absolute square of the wave function, $\left|\Psi\left(x_{0}, t\right)\right|^{2} d x$, which depends on time. 
Sally: I agree that the probability density for measuring position depends on $\left|\Psi\left(x_{0}, t\right)\right|^{2}$.

But when you calculate $\left|\Psi\left(x_{0}, t\right)\right|^{2}$, the time dependent phase factors will cancel out and the probability density will be time independent. You and I have the same probability of measuring the position between $x_{0}$ and $x_{0}+d x$.

Harry: The time-dependent phase factors do not drop out of the cross terms. We need to square the whole wave function, not only the coefficients of $\psi_{1}(x)$ and $\psi_{2}(x)$ separately. That is why we get time dependent cross terms.

With whom do you agree? Explain. Use the simulation "psi1+psi2" to justify your answer. (In this simulation, the position eigenfunction is drawn as a narrow function (but not a delta function) due to constraints in the simulation. It is an approximation for a delta function obtained in an ideal position measurement which has an infinitely high peak and infinitesimal width.)

\section{Measurement of energy}

40. Harry and Sally prepare the same initial state wavefunctions $\frac{\psi_{1}(x)+\psi_{2}(x)}{\sqrt{2}}$ from energy eigenfunctions $\psi_{1}(x)$ and $\psi_{2}(x)$ in their labs at time $\boldsymbol{t}=\mathbf{0}$. Harry measures the energy of his electron in a $1 D$ infinite square well at $t=1$ unit and Sally measures the energy of her electron in an identical $1 D$ infinite square well at time $t=3$ units. Consider the following conversation between Harry and Sally.

Harry: The probability that I will measure energy $E_{n}$ is not the same as the probability that you will measure energy $E_{n}$. The probability is determined by the absolute square of the wavefunction, $|\Psi(x, t)|^{2}$, which depends on time. 
Sally: No. The probability of measuring position depends on the absolute square of the wave function. This time we are measuring energy. The time-dependent phase factors will cancel out because only one factor $e^{-i E_{n} t / \hbar}$ can contribute in calculating the probability of measuring a particular energy $E_{n}$. Thus, the probability of obtaining $E_{n}$ will be time independent. You and I have the same probability of measuring energy $E_{n}$.

Harry: But there will be cross terms in the square of the wave function. The phase factors do not drop out for the cross terms.

Sally: I disagree. The probability of measuring energy is determined by the square of the coefficients of each of the energy eigenfunctions $\psi_{1}(x)$ and $\psi_{2}(x)$. We do not square the entire wavefunction, we only square the coefficients of each energy eigenfunction and the time dependence drops out. For example, the probability of measuring energy $E_{1}$ is given by: $p\left(E_{1}\right)=\left|\frac{e^{-i E_{1} t / \hbar}}{\sqrt{2}}\right|^{2}=\frac{1}{2}$, which is time independent.

With whom do you agree? Explain.

\section{Simulation 9* (Complete if time is available)}

If you are not sure about the answer to question 40 , you may check it with the simulation. Measure the energy at $t=1$ unit for 20 trials, and estimate the probability of obtaining $E_{1}$. Then measure the energy at $t=3$ units for 20 trials and estimate the probability of obtaining $E_{1}$. Combine your data with other groups' to make the result statistically reliable. 


\section{Consecutive measurements}

Measure the energy of the system first and then measure the energy again.

41. At time $t=0$, the initial state of the particle is $\frac{1}{\sqrt{2}}\left(\left|\psi_{1}\right\rangle+\left|\psi_{2}\right\rangle\right)$. We first measure the energy of the particle at time $t$ and obtain the energy $E_{1}$. Then we immediately measure the energy again. What result can we obtain in the second measurement? Explain your choice.
A. Only $E_{1}$
B. Either $E_{1}$ or $E_{2}$
C. Only $E_{1} e^{-i E_{1} t / \hbar}$
D. Either $E_{1} e^{-i E_{1} t / \hbar}$ or $E_{2} e^{-i E_{2} t / \hbar}$
E. Any of $E_{n}, \mathrm{n}=1,2,3, \ldots$

42. In the previous problem (question 41), after the second measurement of energy, what state will the particle be in?
A. $\left|\psi_{1}\right\rangle$ or $\left|\psi_{2}\right\rangle$
B. Any of $\left|\psi_{n}\right\rangle, \mathrm{n}=1,2,3, \ldots$
C. $\left|\psi_{1}\right\rangle$
D. $\frac{1}{\sqrt{2}}\left(\left|\psi_{1}\right\rangle+\left|\psi_{2}\right\rangle\right)$
E. None of the above

\section{Simulation 10}

Choose the simulation "psi1+psi2". The initial state in this simulation is $\frac{1}{\sqrt{2}}\left(\left|\psi_{1}\right\rangle+\left|\psi_{2}\right\rangle\right)$.

Start the time evolution. Around $t=2$ units, first click the start/stop button to pause the time evolution and then measure the energy. What state do you obtain? Then measure the energy again without re-initializing the wavefunction. Is the state the same as the state you observed after your first measurement? 
43. In question 41, if the time interval between the first and second energy measurement is $\Delta t>0$, what is the measured energy and state of the particle after the second measurement?

\section{Simulation 11}

Choose the simulation "psi1+psi2”. The initial state in this simulation is $\frac{1}{\sqrt{2}}\left(\left|\psi_{1}\right\rangle+\left|\psi_{2}\right\rangle\right)$.

Start the time evolution. Around $t=2$ units, first click the start/stop button to pause the time evolution and then measure the energy. What state do you obtain? Then start the time evolution and measure the energy again at $t=3$ units without re-initializing the wavefunction. Is the state the same as your first measurement? (Note that the clock would return to zero when you restart the time evolution.)

First measure the energy of the system and then measure the position after the energy measurement. .

44. At time $t=0$, the initial state of the particle is $\frac{1}{\sqrt{2}}\left(\left|\psi_{1}\right\rangle+\left|\psi_{2}\right\rangle\right)$. We first measure the energy of the particle at time $t=t_{0}$ and obtain the result $E_{1}$. Then we immediately measure the position of the particle (also at time $t=t_{0}$ ). What is the probability of finding the particle in the region between $x_{0}$ and $x_{0}+d x$ ?

45. In the previous problem (question 44), if the measurement of position is made at $t=t_{1}$ instead of $t_{0}$ (not immediately after the energy measurement), what is the probability of finding the particle in the region between $x_{0}$ and $x_{0}+d x$ ? If the particle is found at $x=x_{0}$, what is the state of the particle after the position measurement in Dirac notation and in the position representation? 


\section{Simulation 12}

- Choose the simulation "psi1+psi2". The initial state in this simulation is $\frac{1}{\sqrt{2}}\left(\left|\psi_{1}\right\rangle+\left|\psi_{2}\right\rangle\right)$. Start the time evolution. Around $t=2$ units, first click the start/stop button to pause the time evolution and then measure the energy. What state do you obtain? Explain

- Start the time evolution. Does the shape of the absolute value of wave function change with time? According to your wave function, what is the most probable position for finding the particle? Does this most probable position change with time? Explain

First measure the position of the system and then measure the position again.

46. We first measure the position of a particle in a 1-D infinite square well at time $t=0$ and find the particle at $x=x_{0}$. At time $t(>0)$ after the position measurement, what state will the particle be in? Write your answer in terms of an expansion in a complete set of energy eigenstates. Use $\psi_{n}$ and $E_{n}$ to denote the energy eigenstates and energy eigenvalues. (Hint: refer to question 37)

47. In question 46 , when we make a second measurement of position at time $t(>0)$, what is the probability density of finding the particle at $x=x_{0}$ ? Does the probability density depend on time $t$ when the measurement was performed?

48. In question 46, if the second measurement of position is made immediately after the first position measurement at time $t=0$, what is the probability density of finding the particle at $x=x_{0}$ ?

\section{Simulation 13}

- Choose the simulation "psi1+psi2". The initial state in this simulation is $\frac{1}{\sqrt{2}}\left(\left|\psi_{1}\right\rangle+\left|\psi_{2}\right\rangle\right)$. Start the time evolution. Around $t=2$ units, first click the start/stop button to pause the time evolution and then measure the position. What state do you obtain? Then measure the position again after the first position measurement without starting the time evolution, will you obtain the same state as the first position measurement? Explain. 
- Then start the time evolution. Does the shape of wave function change with time? Will the wave function go back to the state $\frac{1}{\sqrt{2}}\left(\left|\psi_{1}\right\rangle+\left|\psi_{2}\right\rangle\right)$ ? According to your wave function at $t=10$ units, what is the most probable position for finding the particle? Does this most probable position change with time? Explain.

(Note that if you make the second position measurement immediately after the first position measurement, you may find that the wavefunction after the second measurement shifts its position somewhat. This is because the wavefunction in which the system collapses after the position measurement in our simulation is not an ideal position eigenfunction (it is not a delta function). If we had a delta function, the position eigenfunction would be highly localized and the second measurement of position in immediate succession would give us the same result as the first position measurement.)

First measure the position of the system and then measure the energy after the position measurement.

49. Suppose we measure the position of a particle for the initial state $\frac{1}{\sqrt{2}}\left(\left|\psi_{1}\right\rangle+\left|\psi_{2}\right\rangle\right)$ in a 1-D infinite square well at time $t=0$ and find the particle at $x=x_{0}$. Then we measure the energy of the particle immediately after the position measurement. What is the probability of obtaining the ground state energy? (Hint: In order to find the probability of measuring energy, the wavefunction must be expanded in term of a complete set of energy eigenstates.)

50. In question 49, if we perform the measurement of energy after time $t=t_{0}$, what is the probability of measuring the ground state energy? Is the result the same as the result for the immediate energy measurement? (Hint: Find the wavefunction after time $t$ and then calculate the probability of measuring ground state energy.) 


\section{Simulation 14}

- Choose the simulation "psi1+psi2". The initial state in this simulation is $\frac{1}{\sqrt{2}}\left(\left|\psi_{1}\right\rangle+\left|\psi_{2}\right\rangle\right)$. Start the time evolution. Around $t=2$ units, first click the start/stop button to pause the time evolution and then measure the position. Draw the shape of the wavefunction you obtain after the position measurement. Is this what you expected?

- Then measure the energy without restarting the time evolution. Can you predict what energy you will obtain (which energy eigenstate your system will collapse to) after the energy measurement? Will you obtain any energy eigenstates other than $\left|\psi_{1}\right\rangle$ and $\left|\psi_{2}\right\rangle$ ? Explain.

Answer to question 38

The Hamiltonian governs the time evolution of the system according to the time dependent Schrödinger equation (TDSE). Since energy eigenstates $\left|\psi_{n}\right\rangle$ are eigenstates of the $\hat{H}$ operator, the energy eigenstates have a simple time evolution of the form $\left|\psi_{n}\right\rangle e^{-i E_{n} t / \hbar}$. When we write a general state as a superposition of the energy eigenstates (or stationary states), each term in the superposition evolves according to a different phase of the type $e^{-i E_{n} t / \hbar}$ (assuming no degeneracy) so that the state at time $t$ is $\sum_{n} A_{n}\left|\psi_{n}\right\rangle e^{-i E_{n} t / \hbar}$ where $A_{n}$ can be calculated by using the Fourier trick in position representation or by projecting the initial state along the energy eigenstate $\left|\psi_{n}\right\rangle$. 


\section{APPENDIX B}

\section{QUILT FOR STERN-GERLACH EXPERIMENT* (CHAPTER 6)}

* The warm-up and homework parts of the tutorial for Stern-Gerlach experiment are not included in Appendix B.

In this tutorial, we will learn about the basics of quantum mechanics via Stern-Gerlach experiment and use simulations to check the results of Stern-Gerlach experiment after making predictions. Let's do some practice first.

\section{Prediction:}

A beam of atoms in the initial state $|\uparrow\rangle_{z}$ passes the SGZ- (magnetic field gradient in the $-\mathrm{z}$ direction). Two detectors are placed after the SGZ- to count the atoms coming out of the upper and lower channel. What is the probability that each detector clicks when an atom passes? (The pictorial representations of the Stern-Gerlach apparatus are plotted in Figure 6.8 (Section 6.8), which is also a part of the QuILT for Stern-Gerlach experiment. It is not reproduced here.) 


\section{Simulation:}

Double click the green arrow "z-up pass SGZ-" on the left column to check your answer. On the top of the simulation window, click the green button "GO" to start the simulation and click the red button "STOP" to pause the simulation. The "RESET" button clears all the detector counts to zero. The buttons "STEP1"/“STEP1000" send 1 or 1000 particles, respectively, through the SGA (Stern-Gerlach apparatus).

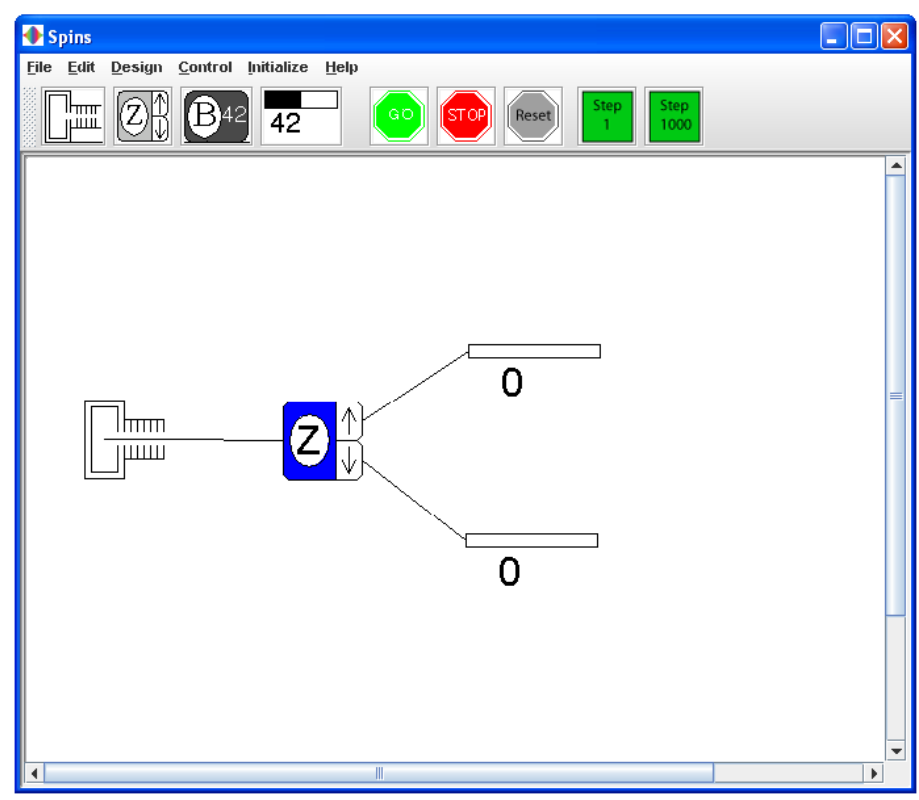

Now let's get started with the tutorial. Note that for all the SGAs used in the simulation, the magnetic field gradient is always in the negative direction. The gradient directions of SGX, SGY and SGZ shown in the simulation are along $-x,-y$ and $-z$ axes. So they will deflect the spin-up state to the upper channel and the spin-down state to the lower channel. 
First predict the answers to the following problem. After that, the tutorial will provide systematic guidance in solving this problem.

You send silver atoms in an initial spin state $|\uparrow\rangle_{z}$ one at a time through two SGAs with magnetic field gradients as shown below. Suitable detectors are placed as shown. One detector is between the two SGAs (in the lower channel) and the other after both SGAs (in the upper channel). What is the probability that a given single atom will cause the "up" detector to click after passing through this system of two SGAs? What is the spin state of the atoms collected in the lower channel after SGZ-?

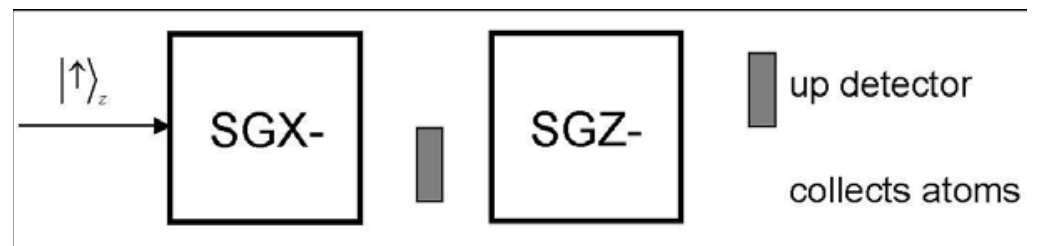

Step1: Write the initial state in a basis most suitable for analyzing the effects of passing through SGX-. [Hint: The time evolution of a system is convenient to analyze choosing the energy eigenstates as the basis vectors. If the Hamiltonian $\hat{H}$ commutes with the $\hat{S}_{x}$, the energy eigenstates are $|\uparrow\rangle_{x}$ and $\left.|\downarrow\rangle_{x}\right]$.

1. Which one of the following gives the correct relationship between the normalized eigenstates of $\hat{S}_{z}$ and $\hat{S}_{x}$.
A. $|\uparrow\rangle_{z}=\frac{1}{\sqrt{2}}\left(|\uparrow\rangle_{x}+i|\downarrow\rangle_{x}\right),|\downarrow\rangle_{z}=\frac{1}{\sqrt{2}}\left(|\uparrow\rangle_{x}-i|\downarrow\rangle_{x}\right)$
B. $|\uparrow\rangle_{z}=\frac{1}{\sqrt{2}}\left(|\uparrow\rangle_{x}+|\downarrow\rangle_{x}\right),|\downarrow\rangle_{z}=\frac{1}{\sqrt{2}}\left(|\uparrow\rangle_{x}-|\downarrow\rangle_{x}\right)$
C. $|\uparrow\rangle_{z}=\frac{1}{\sqrt{2}}\left(a|\uparrow\rangle_{x}+b|\downarrow\rangle_{x}\right),|\downarrow\rangle_{z}=\frac{1}{\sqrt{2}}\left(a|\uparrow\rangle_{x}-b|\downarrow\rangle_{x}\right)$, where $a$ and $b$ can be any complex numbers that satisfy $|a|^{2}+|b|^{2}=1$

D. $|\uparrow\rangle_{z}=|\downarrow\rangle_{x},|\downarrow\rangle_{z}=|\uparrow\rangle_{x}$ 
2. John sends silver atoms in the $|\uparrow\rangle_{z}$ state through an SGX-. He places a "down" detector to block some silver atoms and collects the atoms coming out in the "upper channel". Which one of the following normalized spin states has John prepared in the "upper channel"? Think about how you can use the SGAs to check the state. Draw a figure below and explain. Hint: if all of the atoms passing through an SGX are collected by the upper (or lower) detector, the spin state of the atoms is purely $|\uparrow\rangle_{x}$ (or $|\downarrow\rangle_{x}$ ).
A. $|\uparrow\rangle_{z}$
B. $|\uparrow\rangle_{x}$
C. $\frac{1}{2}|\uparrow\rangle_{z}$
D. $\frac{1}{\sqrt{2}}|\uparrow\rangle_{2}$

㑱Simulation: Now use the two simulations "z-up pass SGX-1" and "z-up pass SGX-2" to check your answers. In the first simulation, the atoms prepared in the "upper channel" passed through a SGZ-. In the second simulation, the atoms prepared in the upper channel passed through a SGX-. Is your prediction in question 2 consistent with the observations in these simulations? If not, reconcile the difference.

Step2: Find the fraction of atoms that would pass through the second SGA (which were not absorbed by the first detector).

3. In question 2, what is the probability of the "down" detector clicking when John sends a silver atom? Does this probability depend on how much time the atom has stayed in the nonuniform magnetic field so long as the detectors are placed in appropriate locations after the SGA? Explain.
A. 1
B. 0
C. 0.5
D. It is between 0 and 0.5 , but the exact probability cannot be inferred from the given information. 
Simulation: After you have predicted the answer to the previous question, click on the simulation "z-up pass SGX-" to check your answers. In this simulation, we use detectors in both channels to estimate the probability. But if you want to prepare the atoms in $|\uparrow\rangle_{x}$ or $|\downarrow\rangle_{x}$ state, then you should use only one detector to block the unwanted component.

4. In question 2, if John measures $\hat{S}_{x}$ for the atoms he prepared in the "upper channel”, what is the probability of measuring $+\frac{\hbar}{2}$ ?
A. 1
B. 0
C. 0.5
D. It is between 0 and 0.5 , but the exact probability cannot be inferred from the given information.

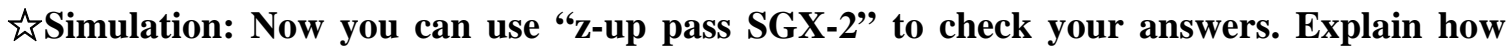
your observation is consistent with your prediction. If it is not consistent, reconcile the difference.

In questions 1 to 4, you have learned the relationship between the eigenstates of $\hat{S}_{z}$ and $\hat{S}_{x}$. Now apply similar ideas to $\hat{S}_{z}$ and $\hat{S}_{y}$. Answer questions 5 to 8 .

5. Which one of the following gives the correct relationship between the normalized eigenstates of $\hat{S}_{z}$ and $\hat{S}_{y}$.
A. $|\uparrow\rangle_{z}=\frac{1}{\sqrt{2}}\left(|\uparrow\rangle_{y}+i|\downarrow\rangle_{y}\right),|\downarrow\rangle_{z}=\frac{1}{\sqrt{2}}\left(i|\uparrow\rangle_{y}-|\downarrow\rangle_{y}\right)$
B. $|\uparrow\rangle_{z}=\frac{1}{\sqrt{2}}\left(|\uparrow\rangle_{y}-i|\downarrow\rangle_{y}\right),|\downarrow\rangle_{z}=\frac{-i}{\sqrt{2}}\left(|\uparrow\rangle_{y}+i|\downarrow\rangle_{y}\right)$
C. $|\uparrow\rangle_{z}=\frac{1}{\sqrt{2}}\left(a|\uparrow\rangle_{x}+b|\downarrow\rangle_{x}\right),|\downarrow\rangle_{z}=\frac{1}{\sqrt{2}}\left(a|\uparrow\rangle_{x}-b|\downarrow\rangle_{x}\right)$, where $a$ and $b$ can be any complex numbers that satisfy $|a|^{2}+|b|^{2}=1$
D. $|\uparrow\rangle_{z}=|\downarrow\rangle_{y},|\downarrow\rangle_{z}=|\uparrow\rangle_{y}$ 
6. Check that ${ }_{z}\langle\uparrow \mid \downarrow\rangle_{z}=0$ by expressing $|\uparrow\rangle_{z}$ and $|\downarrow\rangle_{z}$ in the $\hat{S}_{y}$ basis as above.

7. John sends silver atoms in the $|\uparrow\rangle_{z}$ state through a SGY-. He places a "down" detector to block some silver atoms and collects the atoms coming out in the "upper channel". What is the probability of the "down" detector clicking for each atom sent when John sends the silver atoms?
A. 1
B. 0
C. 0.5
D. It is between 0 and 0.5 , but the exact probability cannot be inferred from the given information.

话Simulation: Now you can click on the simulation “z-up pass SGY-” to check your answers. Explain how your observation is consistent with your prediction. If it is not consistent, reconcile the difference.

8. In the previous experiment, which one of the following normalized spin states has John prepared in the "upper channel”?
A. $|\uparrow\rangle_{z}$
B. $|\uparrow\rangle_{y}$
C. $\frac{1}{2}|\uparrow\rangle_{y}$
D. John has not prepared anything. Everything gets blocked by the "down” detector.

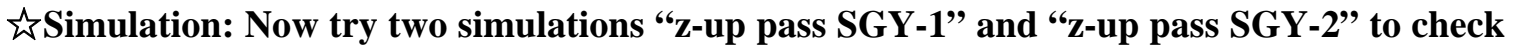
your answers. In the first simulation, the atoms prepared in the "upper channel" passed through a SGZ-. In the second simulation, the atoms prepared in the upper channel passed through a SGX-. Based upon the observations in the two simulations, is your prediction in question 8 consistent with the simulation? 


\section{Step3: As in Step 1, write the spin state of atoms before SGZ in a proper basis which}

\section{helps to analyze the time evolution in SGZ.}

9. The "down" detector between SGX and SGZ will collapse the state of the silver atoms. If the detector clicks, the atom gets absorbed by the detector. If the detector does not click, write down the spin state after passing through the SGX right before entering SGZ. Express this spin state in a basis that is most suitable for determining the time evolution after the atoms have passed through the SGZ.

\section{Now let's solve the problem given at the beginning of this tutorial.}

10. You send silver atoms in an initial spin state $|\uparrow\rangle_{z}$ one at a time through two SGAs with magnetic field gradients as shown below. Suitable detectors are placed as shown. One detector is between the two SGAs (in the lower channel) and the other after both SGAs (in the upper channel). What is the probability that a given single atom will cause the "up" detector to click after passing through this system of two SGAs?

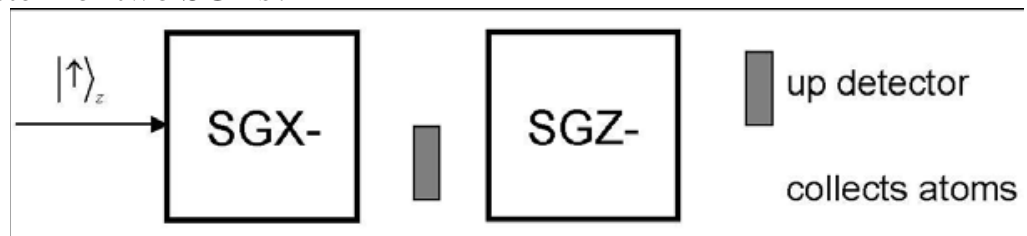
A. 1
B. 0
C. 0.5
D. 0.25

话Simulation : Now you can click the simulation "z-up pass SGX-1" to check your answer. Note that instead of collecting atoms, we have put detectors in both the upper and lower channels at the end to estimate the probability. Explain whether your prediction is consistent with the observation.

11. Consider the following conversation between Andy and Caroline:

Andy: I don't understand the answer to the previous question (question 10).

Caroline: When an atom in the state $|\uparrow\rangle_{z}=\frac{1}{\sqrt{2}}\left(|\uparrow\rangle_{x}+|\downarrow\rangle_{x}\right)$ passes through the SGX, each eigenstate of $\hat{S}_{x}$ gets spatially separated. If the detector between SGX and SGZ does not click, the state of 
that silver atom must have collapsed to $|\uparrow\rangle_{x}$. Since the atom in this state passes through SGZ next, we must write $|\uparrow\rangle_{x}=\frac{1}{\sqrt{2}}\left(|\uparrow\rangle_{z}+|\downarrow\rangle_{z}\right)$. You can see that $|\uparrow\rangle_{x}$ is a superposition of the eigenstates of $\hat{S}_{z}$ with equal weight to $|\uparrow\rangle_{z}$ and $|\downarrow\rangle_{z}$. This helps us find the probability that the second detector clicks.

Andy: Is an eigenstate of any one component of spin, say $|\uparrow\rangle_{y}$ for $\hat{S}_{y}$, a superposition of the eigenstates of any of the other two components, say $\hat{S}_{z}$ or $\hat{S}_{x}$, with equal weight?

Caroline: Yes. There may be a phase factor such as "i” (where $i=\sqrt{-1}$ ) when you write $|\uparrow\rangle_{y}$ in terms of the superposition of $|\uparrow\rangle_{z}$ and $|\downarrow\rangle_{z}$ but the probability is the same for both eigenstates of $\hat{S}_{z}$.

Do you agree with Caroline? Explain.

12. In the previous experiment (question 10), you collect the silver atoms that are not blocked at the end after they have passed through both SGAs. Which one of the following is the spin state of the silver atom you collect at the end in the lower channel?
A. $|\uparrow\rangle_{z}$
B. $|\downarrow\rangle_{z}$
C. $|\downarrow\rangle_{x}$
D. You do not collect anything because all atoms passing through the second SGA are blocked by the detector

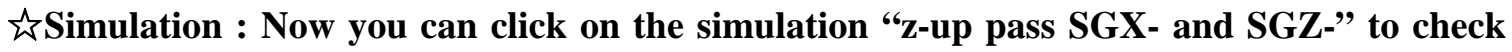
your answers. Explain any discrepancy between your prediction and observation. Note that the SGZ is only inserted to check the final state. 
13. Consider the following conversation between Andy and Caroline:

Andy: There must be something wrong with the answer to the previous question (question 12).

How can the $|\uparrow\rangle_{z}$ that we inputted give $|\downarrow\rangle_{z}$ on the way out?

Caroline: I disagree. If you let atoms in the state $|\uparrow\rangle_{z}$ pass through SGZ only, you will never obtain $|\downarrow\rangle_{z}$ on the way out. However, $|\downarrow\rangle_{z}$ is obtained in the above experiment because we have inserted SGX at an intermediate stage. Think of the analogy with vertically polarized light passing directly through a horizontal polarizer vs. passing first through a polarizer at $45^{\circ}$ followed by a horizontal polarizer. There is no light at the output if vertically polarized light passes directly through a horizontal polarizer. On the other hand, if the polarizer at $45^{\circ}$ is present, light becomes polarized at $45^{\circ}$ after the $45^{\circ}$ polarizer which is a linear superposition of horizontal and vertical polarization. Therefore, some light comes out through the horizontal polarizer placed after the $45^{\circ}$ polarizer.

Do you agree with Caroline? Explain. Also, comment on how good is the analogy between the spin-1/2 state of the atoms and the polarization state of photons.

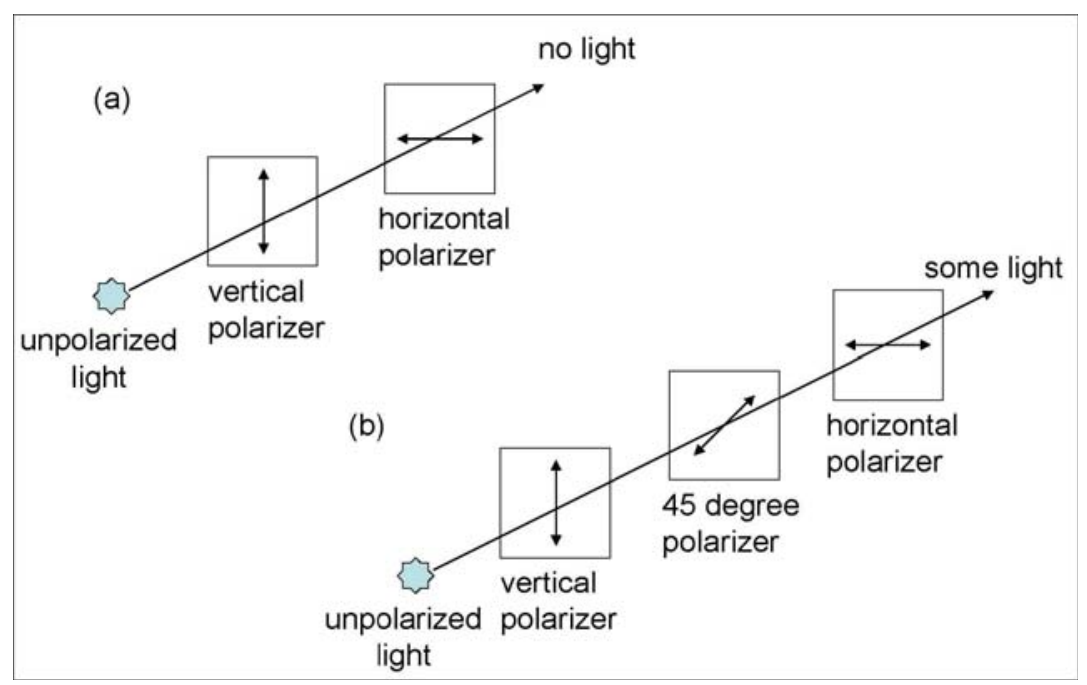


Now consider the following problem. Questions 14 to 16 provide the steps to solve this problem.

Consider two situations as below.

Situation 1: The beam of atoms is in the pure state $\frac{1}{\sqrt{2}}\left(|\uparrow\rangle_{z}+|\downarrow\rangle_{z}\right)$.

Situation 2: The beam of atoms is an unpolarized mixture, half of which is $|\uparrow\rangle_{z}$ and the other half $|\downarrow\rangle_{z}$.

Design an experiment to differentiate these two beams of atoms. (You should be able to tell after your experiment that one of the beams is in a pure state and the other is a mixture.)

14. Read the following statement and answer the questions.

Andy: There is no difference between silver atoms in a "pure" state given by $\frac{1}{\sqrt{2}}\left(|\uparrow\rangle_{z}+|\downarrow\rangle_{z}\right)$ and an unpolarized mixture in which half of the atoms are in the $|\uparrow\rangle_{z}$ state and half are in the $|\downarrow\rangle_{z}$ state. If we had sent atoms in the superposition state $\frac{1}{\sqrt{2}}\left(|\uparrow\rangle_{z}+|\downarrow\rangle_{z}\right)$ through the SGZ, half of them would have registered in the "up" detector and half of them would have been collected in the lower channel. The outcome will be exactly the same if we had sent a 50/50 mixture of $|\uparrow\rangle_{z}$ and $|\downarrow\rangle_{z}$ through the SGZ. So there is no way to distinguish a mixture from a superposition.

Question : Is the statement above correct? Explain.

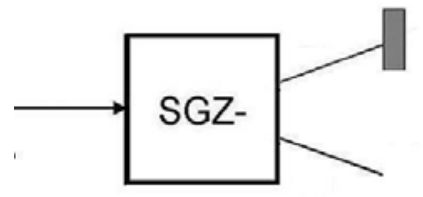


15. Remember the analogy between spin states and polarized photons in question 13. Suppose you have a beam of pure polarized photons with 45 degrees polarization and another beam of unpolarized mixture with half of the photons vertically polarized and half horizontally polarized. Will a vertical or horizontal polarizer tell you which beam is in a pure state? What polarizer could you use to differentiate the two beams of photons?

16. Based upon the analogy for distinguishing between pure polarized photons and a beam of photon mixture, what kind of SGA could you use to differentiate the two beams of atoms in question 14? Draw a sketch below to explain your choice. Do not forget to put the detectors in the correct positions. (Hint: Use the simulations " $z$-up pass SGX" and "z-up+z-down pass SGX" to check your answer. In the simulation "z-up+z-down pass SGX", the incoming particles are in the pure state $\frac{1}{\sqrt{2}}\left(|\uparrow\rangle_{z}+|\downarrow\rangle_{z}\right)$.)

\section{Questions 18 and 19 relate to the simulation “unknown state”. Run the simulation}

\section{"unknown state" first. Then answer the following questions.}

18. Write down at least 3 different possible spin states of the incoming particles that will show the behavior seen in the simulation. The incoming particles do not necessarily have identical spin states. Explain your reasoning for your choices.

19. Choose two of the different possible spin states you predicted for the simulation you saw. Now come up with some simulations using SGAs that would distinguish between the two possible spin states. You can choose one or more SGAs to find out which of the two spin states it is. Share your set-up with others in your class. 


\section{Instructions on building your own SGA:}

(1) Open the simulation "unknown state” or any other existing simulation.

(2) Choose "File $\rightarrow$ New" on the simulation menus. Then you would have a white board.

(3) Click the first button "New Gun" to add a particle source to the white board. You can click the icon on the white board and drag it to a new position.

(4) Choose "Initialize $\rightarrow$ User State" to set up the initial spin state of the particle source. You can choose the basis X, Y or Z regarding spin Sx, Sy and Sz. And then you can input the coefficient of spinup and spin-down in the table. Take Sx as an example.

\begin{tabular}{|c|c|c|c|c|}
\hline System & Real 1 & Imag 1 & Real 2 & Imag 2 \\
\hline Spin $1 / 2$ & 1 & 2 & 3 & 4 \\
\hline
\end{tabular}

The coefficient in the table above means the initial state is $(1+2 i)\left|\uparrow_{x}\right\rangle+(3+4 i)\left|\downarrow_{x}\right\rangle$

*Remember to press "Enter” on the keyboard after you have input all the numbers.

5. Click the second button "New Analyzer" to add the Stern-Gerlach apparatus. The letter "X”, "Y" and "Z" represent the direction of the magnetic field gradient. You can change the letter by clicking it. The letter " $n$ ” represent a customized direction which could be defined by "Design $\rightarrow$ Change Angles".

6. Click the fourth button "New Counter" to add the detector. Drag it to the proper position.

7. Connect the particle source and the SGA by clicking the end on the right side of the particle source and dragging a line to the left side of the SGA. Then click the upper or lower channel of the SGA and drag a line to the detector to connect them together.

8. Click "Go" to test whether the simulation works well. 
APPENDIX C

\section{QUANTUM MECHANICS SURVEY (CHAPTER 9)}




\section{Quantum Mechanics Survey}

\section{Instructions for all of the questions}

$* \hat{x}, \hat{p}$ and $\hat{H}$ correspond to the position, momentum and Hamiltonian operators, respectively, for a given quantum system.

* A physical observable is "well-defined" when it has a definite value for a given wavefunction.

* "1D" is an abbreviation for "one dimensional". All questions refer to systems in one spatial dimension.

* The wavefunction at time $t$ is written as $\Psi(x, t)$

System I: A particle interacts with a one-dimensional infinite square well of width $a(V(x)=0$ for $0 \leq x \leq a$ and $V(x)=+\infty$ otherwise) as shown. The stationary states are $\psi_{n}(x)=\sqrt{\frac{2}{a}} \sin (n \pi x / a)$ and the allowed energies are $E_{n}=\frac{n^{2} \pi^{2} \hbar^{2}}{2 m a^{2}}$ where $n=1,2,3, \ldots, \infty$.

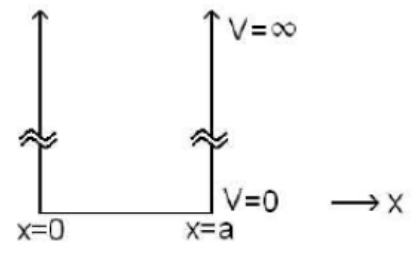

Infinite Square Well

Questions 1 through 15 relate to System I, shown above:

1. Choose all of the following wave functions that are possible for System I at time $t=0$.

$\Psi(x, 0)$ and $d \Psi(x, 0) / d x$ are both continuous and single-valued in the region $0<x<a$

for the states shown below.

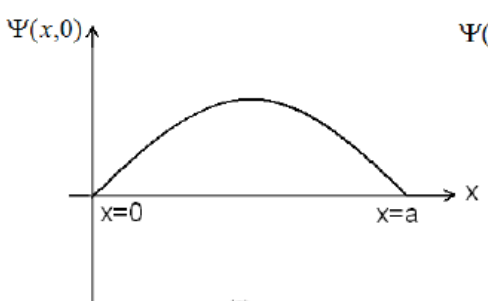

(I)

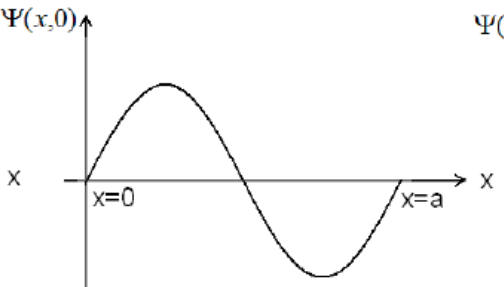

(II)

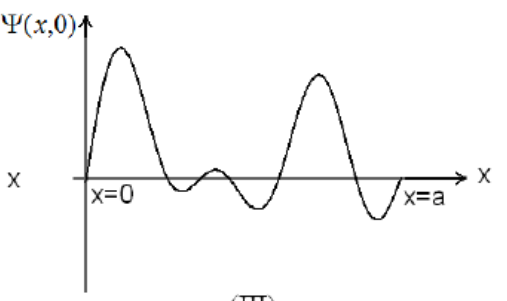

(III)
A. None of the above
B. All of the above
C. (I) only
D. (I) and (II) only
E. (I) and (III) only

2. Suppose that at time $t=0$, System I is in the first excited state. Choose all of the following expectation value(s) that depend on time.
(1) $\langle x\rangle$
(2) $\langle p\rangle$
(3) $\langle H\rangle$
A. 1 only
B. 2 only
C. 3 only
D. 1 and 2 only
E. None of the above 
System I: A particle interacts with a one-dimensional infinite square well of width $a(V(x)=0$ for $0 \leq x \leq a$ and $V(x)=+\infty$ otherwise) as shown. The stationary states are $\psi_{n}(x)=\sqrt{\frac{2}{a}} \sin (n \pi x / a)$ and the allowed energies are $E_{n}=\frac{n^{2} \pi^{2} \hbar^{2}}{2 m a^{2}}$ where $n=1,2,3, \ldots, \infty$.

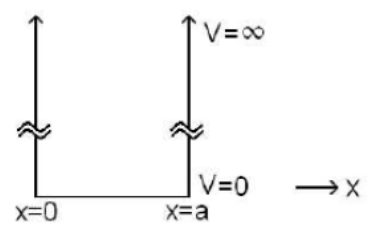

Infinite Square Well

3. Consider the following wavefunction: $\Psi(x, 0)=\sqrt{2 / a} \sin (5 \pi x / a)$. Which one of the following is the probability density $|\Psi(x, t)|^{2}$, at time $t$ ?
A. $|\Psi(x, t)|^{2}=\frac{2}{a} \sin ^{2}(5 \pi x / a) \cos ^{2}\left(E_{5} t / \hbar\right)$ time-independent.
B. $|\Psi(x, t)|^{2}=\frac{2}{a} \sin ^{2}(5 \pi x / a) \exp \left(-i 2 E_{5} t / \hbar\right)$
E. None of the above.
C. $|\Psi(x, t)|^{2}=\frac{2}{a} \sin ^{2}(5 \pi x / a) \sin ^{2}\left(E_{5} t / \hbar\right)$

D. $|\Psi(x, t)|^{2}=\frac{2}{a} \sin ^{2}(5 \pi x / a)$ which is

4. Consider the following wavefunction: $\Psi(x, 0)=A \sin ^{5}(\pi x / a)$, where $A$ is a suitable normalization constant. Which one of the following is the probability density $|\Psi(x, t)|^{2}$, at time $t$ ?
A. $|\Psi(x, t)|^{2}=|A|^{2} \sin ^{10}(\pi x / a) \cos ^{2}\left(E_{5} t / \hbar\right)$ time-independent.
B. $|\Psi(x, t)|^{2}=|A|^{2} \sin ^{10}(\pi x / a) \exp \left(-i 2 E_{5} t / \hbar\right)$
E. None of the above.
C. $|\Psi(x, t)|^{2}=|A|^{2} \sin ^{10}(\pi x / a) \sin ^{2}\left(E_{5} t / \hbar\right)$

D. $|\Psi(x, t)|^{2}=|A|^{2} \sin ^{10}(\pi x / a)$ which is

5. Suppose the particle for System I is in the ground state with wavefunction $\psi_{1}(x)$. Which one of the following is the probability that the particle will be found in a narrow range between $x$ and $x+d x$ ?
A. $\left|\psi_{1}(x)\right|^{2} d x$
B. $x\left|\psi_{1}(x)\right|^{2} d x$
D. $\int_{-\infty}^{+\infty} x\left|\psi_{1}(x)\right|^{2} d x$
E. None of the above.
C. $\quad \int_{x}^{x+d x} x\left|\psi_{1}(x)\right|^{2} d x$ 
System I: A particle interacts with a one-dimensional infinite square well of width $a(V(x)=0$ for $0 \leq x \leq a$ and $V(x)=+\infty$ otherwise) as shown. The stationary states are $\psi_{n}(x)=\sqrt{\frac{2}{a}} \sin (n \pi x / a)$ and the allowed energies are $E_{n}=\frac{n^{2} \pi^{2} \hbar^{2}}{2 m a^{2}}$ where $n=1,2,3, \ldots, \infty$.

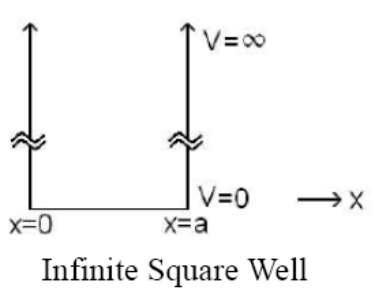

6. Choose all of the following statements that are correct for System I. In all the options, $A_{n}$ are suitable expansion coefficients such that the wavefunction is normalized and at least two $A_{n}$ are nonzero.

(1) $\Psi(x, 0)=\sum_{n} A_{n} \psi_{n}(\mathrm{x})$ is a possible wavefunction for the particle at time $t=0$.

(2) $\Psi(x, 0)=\sum_{n} A_{n} \psi_{n}(\mathrm{x})$ satisfies the time independent Schrödinger equation, $\hat{H} \Psi(x, 0)=E \Psi(x, 0)$.

(3) $\Psi(x, t)=\sum_{n} A_{n} \psi_{n}(\mathrm{x}) \exp \left(-i E_{n} t / \hbar\right)$ satisfies the time dependent Schrödinger equation, $i \hbar \frac{\partial \Psi(x, t)}{\partial t}=\hat{H} \Psi(x, t)$
A. 1 only
B. 2 and 3 only
C. 1 and 2 only
D. 1 and 3 only
E. All of the above

7. The wavefunction for System I at time $t=0$ is $\sqrt{2 / 7} \psi_{1}(x)+\sqrt{5 / 7} \psi_{2}(x)$ when you perform a measurement of energy. The energy measurement yields $4 \pi^{2} \hbar^{2} / 2 m a^{2}$. Which one of the following is the spatial part of the normalized wavefunction (excluding the time part) after the energy measurement? (Ignore the overall phase of the wavefunction.)
(1) $\psi_{2}(x)$
(2) $\sqrt{5 / 7} \psi_{2}(x)$
(3) $\sqrt{2 / 7} \psi_{1}(x)+\sqrt{5 / 7} \psi_{2}(x)$
A. 1 only
B. 2 only
C. 3 only

D. Depends on how long you wait after the energy measurement. At the instant energy is measured, it will be (1) but a long time after that it will be (3).

E. Depends on how long you wait after the energy measurement. At the instant energy is measured, it will be (2) but a long time after that it will be (3). 


\begin{tabular}{|l|l|} 
System I: A particle interacts with a one-dimensional infinite \\
square well of width $a(V(x)=0$ for $0 \leq x \leq a$ and \\
$V(x)=+\infty$ otherwise $)$ as shown. The stationary states are \\
$\psi_{n}(x)=\sqrt{\frac{2}{a}} \sin (n \pi x / a)$ and the allowed energies are \\
$E_{n}=\frac{n^{2} \pi^{2} \hbar^{2}}{2 m a^{2}}$ where $n=1,2,3, \ldots, \infty$.
\end{tabular}

8. Consider a wavefunction for System I given by $\Psi(x, t=0)=\sqrt{4 / 7} \psi_{1}(x)+\sqrt{3 / 7} \psi_{2}(x)$. Which one of the following statements is true about the measurement of energy for this system?

A. The only possible value of energy measured will be $4 E_{1} / 7+3 E_{2} / 7$.

B. The only possible values measured will be the ground state energy $E_{1}$ or the first excited state energy $E_{2}$.

C. The possible values of energy measured will be $E_{n}=n^{2} \pi^{2} \hbar^{2} /\left(2 m a^{2}\right)$ (where $\mathrm{n}$ is any positive integer) but the probability of measuring $E_{1}$ is largest.

D. The possible values of energy measured will be $E_{n}=n^{2} \pi^{2} \hbar^{2} /\left(2 m a^{2}\right)$ (where $\mathrm{n}$ is any positive integer) and all $E_{n}$ are equally probable.

E. None of the above.

9. Consider the following wavefunction for System I: $\Psi(x, t=0)=\frac{1}{\sqrt{3}} \psi_{1}(\mathrm{x})+i \sqrt{\frac{2}{3}} \psi_{2}(\mathrm{x})$. Choose all of the following statements that are correct about the expectation value of the energy of the system at time $t=0$.

(1) $\langle E\rangle=\frac{1}{3} E_{1}-\frac{2}{3} E_{2}$

(2) $\langle E\rangle=\frac{1}{3} E_{1}+\frac{2}{3} E_{2}$

(3) $\langle E\rangle=\int_{0}^{a} \Psi^{*}(x, 0) \hat{H} \Psi(x, 0) d x$
A. 1 only
D. 1 and 3 only
B. 2 only
E. 2 and 3 only
C. 3 only 
System I: A particle interacts with a one-dimensional infinite square well of width $a(V(x)=0$ for $0 \leq x \leq a$ and $V(x)=+\infty$ otherwise) as shown. The stationary states are $\psi_{n}(x)=\sqrt{\frac{2}{a}} \sin (n \pi x / a)$ and the allowed energies are $E_{n}=\frac{n^{2} \pi^{2} \hbar^{2}}{2 m a^{2}}$ where $n=1,2,3, \ldots, \infty$

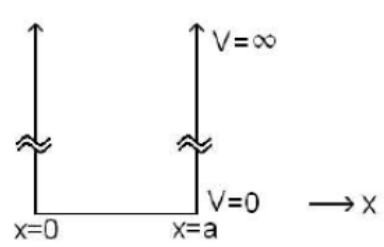

Infinite Square Well

10. Consider the following wavefunction for System I: $\Psi(x, t=0)=\frac{1}{\sqrt{3}} \psi_{1}(\mathrm{x})+i \sqrt{\frac{2}{3}} \psi_{2}(\mathrm{x})$. Choose all of the following statements that are correct about the expectation value of the energy of the system at time $t>0$.

(1) $\langle E\rangle=\frac{1}{3} E_{1} e^{-i E_{1} t / \hbar}-\frac{2}{3} E_{2} e^{-i E_{2} t / \hbar}$

(2) $\langle E\rangle=\frac{1}{3} E_{1} e^{-i E_{1} t / \hbar}+\frac{2}{3} E_{2} e^{-i E_{2} t / \hbar}$

(3) $\langle E\rangle=\int_{0}^{a} \Psi^{*}(x, t) \hat{H} \Psi(x, t) d x$
A. None of the above
D. 3 only
B. 1 only
E. 1 and 3 only
C. 2 only

11. Consider the following wavefunction for System I at time $t=0: \Psi(x, 0)=A x(a-x)$ for $0 \leq x \leq a$ and $\Psi(x, 0)=0$ otherwise. $A$ is a normalization constant. Which one of the following expressions correctly represents the probability of measuring the energy $E_{n}$ in the state $\Psi(x, 0)$ ?
A. $\left|\int_{0}^{a} \psi_{n}^{*}(x) \hat{H} \Psi(x, 0) d x\right|^{2}$
C. $\left|\psi_{n}^{*}(x) \hat{H} \Psi(x, 0)\right|^{2}$
B. $\left|\int_{0}^{a} \psi_{n}^{*}(x) \Psi(x, 0) d x\right|^{2}$
D. $\left|\psi_{n}^{*}(x) \Psi(x, 0)\right|^{2}$
E. $|\Psi(x, 0)|^{2}$ 
System I: A particle interacts with a one-dimensional infinite square well of width $a(V(x)=0$ for $0 \leq x \leq a$ and $V(x)=+\infty$ otherwise) as shown. The stationary states are $\psi_{n}(x)=\sqrt{\frac{2}{a}} \sin (n \pi x / a)$ and the allowed energies are $E_{n}=\frac{n^{2} \pi^{2} \hbar^{2}}{2 m a^{2}}$ where $n=1,2,3, \ldots, \infty$.

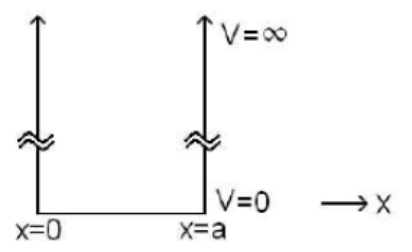

Infinite Square Well

12. Particle $A$ is in a $1 D$ infinite square well (system I) and particle B is in a separate, identical well. At time $t=0$, particle $\mathrm{A}$ is in the state $\frac{1}{\sqrt{2}}\left(\psi_{1}+\psi_{2}\right)$, and particle $\mathrm{B}$ is in the state $\frac{1}{\sqrt{2}}\left(\psi_{1}+i \psi_{2}\right)$. Choose all of the following statements that are correct at a given time $t>0$.

(1) The particles A and B have the same expectation value of position at time $t$.

(2) The particles A and B have the same expectation value of momentum at time $t$.

(3) The particles A and B have the same expectation value of energy at time $t$.
A. 1 only
B. 2 only
C. 3 only
D. All of the above
E. None of the above

13. At time $t=0$, the state for System I is $\frac{1}{\sqrt{2}}\left(\psi_{1}+\psi_{2}\right)$. We first measure the position of the particle at time $t=0$ and obtain the result $x_{0}$. Immediately after the position measurement, we measure the energy. What possible result(s) can we obtain for the energy measurement?

A. We can only measure either $E_{1}$ or $E_{2}$.

B. We can obtain one of the energy values $E_{n}=\frac{n^{2} \pi^{2} \hbar^{2}}{2 m a^{2}}$ where $n$ can be an arbitrarily large integer.

C. We can only measure $\frac{1}{2}\left(E_{1}+E_{2}\right)$.

D. We may measure any energy $E=\sum_{n=1}^{\infty} c_{n} E_{n}$ where $c_{n}$ are coefficients so that $\sum_{n=1}^{\infty}\left|c_{n}\right|^{2}=1$.

E. None of the above. 
System I: A particle interacts with a one-dimensional infinite square well of width $a(V(x)=0$ for $0 \leq x \leq a$ and $V(x)=+\infty$ otherwise) as shown. The stationary states are $\psi_{n}(x)=\sqrt{\frac{2}{a}} \sin (n \pi x / a)$ and the allowed energies are $E_{n}=\frac{n^{2} \pi^{2} \hbar^{2}}{2 m a^{2}}$ where $n=1,2,3, \ldots, \infty$.

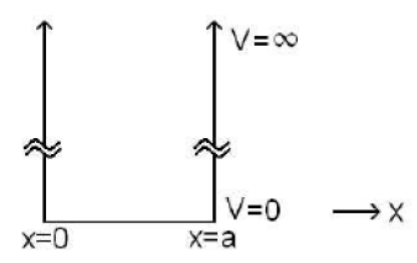

Infinite Square Well

14. At time $t=0$, consider the function $\Psi(x, t=0)=A \sin ^{2}(\pi x / a)$ for $0 \leq x \leq a$ and $\Psi(x, 0)=0$ otherwise. $A$ is a suitable normalization constant. Choose all of the following statements that are correct about whether this function is a possible wavefunction for System I at time $t=0$.

(1) It is not a possible wavefunction because it is not in the form $A \sin (n \pi x / a)$ where $\mathrm{n}=1$, $2,3, \ldots \infty$.

(2) It is not a possible wavefunction because it does not satisfy the time independent Schrödinger equation $H \Psi(x, 0)=E \Psi(x, 0)$.

(3) It is a possible wavefunction for two particles instead of a single particle.
A. 1 only
B. 2 only
C. 3 only
D. 1 and 2 only
E. none of the above

15. A particle is initially in the ground state of an infinite square well with boundaries between $0 \leq x \leq a$. At time $t=0$ the width of the well suddenly increases to $2 a$ (boundaries between $0 \leq x \leq 2 a$ ) so that the wavefunction does not have time to change during the increase in width. Which one of the following statements is correct at time $t>0$ ?

A. The wavefunction of the particle will stay in the ground state of the initial well for all future times even though the well has expanded.

B. The wavefunction of the particle will evolve to the ground state wavefunction of the new well and stay in that state.

C. The wavefunction of the particle will change to the first excited state of the new well and stay in that state.

D. The probability density function of the particle will change with time for all $t>0$.

E. None of the above. 


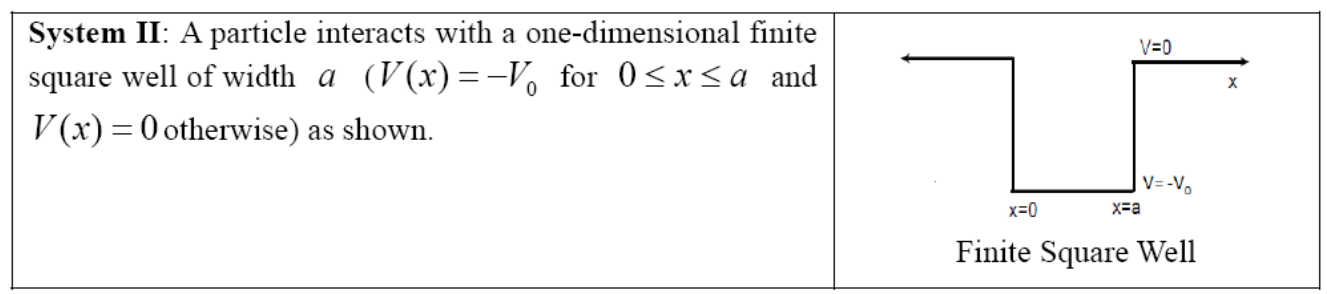

Questions 16 through 19 relate to System II as shown above:

16. Choose all of the following statements that are correct about the wavefunction shown below for system II at time $t=0 . \Psi(x, 0)$ and $d \Psi(x, 0) / d x$ are continuous and single-valued everywhere. The wavefunction $\Psi(x, 0)$ is zero in the regions $x<b_{1}$ and $x>b_{2}$. Assume that the area under the $|\Psi(x, 0)|^{2}$ curve is 1 .

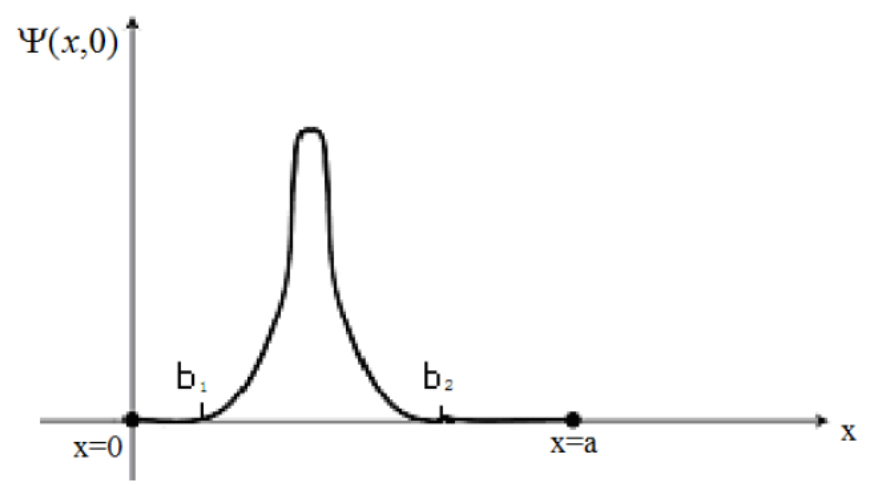

(1) It is a possible wavefunction.

(2) It is not a possible wavefunction because it does not satisfy the boundary conditions. Specifically, it goes to zero inside the well.

(3) It is not a possible wavefunction because the probability of finding the particle outside the finite square well is zero but quantum mechanically it must be nonzero.
A. 1 only
B. 2 only
C. 3 only
D. 2 and 3 only
E. None of the above

17. Suppose you perform a measurement of the position of the particle when it is in the first excited state of system II. Choose all of the following statements that are correct about this experiment:

(1) Right after the position measurement, the wavefunction will be peaked about a particular value of position.

(2) A long time after the position measurement, the wavefunction will go back to the first excited state wavefunction.

(3) The wavefunction will not go back to the first excited state wavefunction even if you wait for a long time after the position measurement.
A. 1 only
B. 2 only
C. 3 only
D. 1 and 2 only
E. 1 and 3 only 


\begin{tabular}{|l|l|}
\hline System II: A particle interacts with a one-dimensional finite \\
square well of width $a\left(V(x)=-V_{0}\right.$ for $0 \leq x \leq a$ and \\
$V(x)=0$ otherwise) as shown. & $\begin{array}{c}\mathrm{x}=0 \quad \\
\text { Finite Square Well }\end{array}$ \\
\hline $\begin{array}{l}\mathrm{v}=-\mathrm{v}_{0} \\
\mathrm{x}\end{array}$
\end{tabular}

18. Choose all of the following statements that are correct for System II, regardless of whether it is in a bound state or a scattering state.

(1) The particle may be found outside the region between $x=0$ and $x=a$.

(2) The energy levels of the system are discrete.

(3) The stationary state wavefunctions may or may not be normalizable but we can make a normalizable wavefunction by using their linear superposition.
A. 1 only
B. 2 only
C. 3 only
D. 1 and 3 only
E. All of the above

19. A particle interacts with a finite square well of width $a$ as shown below. Two possible energies $E_{1}$ and $E_{2}$ are such that $-V_{0}<E_{1}<0$ and $E_{2}>0$. Choose all of the following statements that are correct about a particle in a bound or a scattering state.

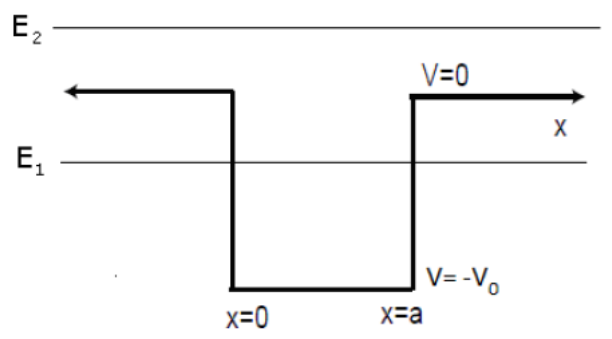

(1) A single particle in a given stationary state can have an energy $E_{1}$ inside the well and have a different energy $E_{2}$ outside the well.

(2) Whether the particle is in a bound or a scattering state depends on the location of the particle if the energy of the particle is $E_{2}$.

(3) Whether the particle is in a bound or a scattering state depends on the location of the particle if the energy of the particle is $E_{1}$.
A. 1 only
B. 2 only
C. 3 only
D. 2 and 3 only
E. none of the above 
System III: A particle with mass $m$ interacts with a one-dimensional simple harmonic oscillator well $V(x)=K x^{2} / 2$ as shown. The stationary states are $\psi_{n}(x)$ and the allowed energies are $E_{n}=(n+1 / 2) \hbar \omega$ where $n=0,1,2,3, \ldots, \infty$ and $\omega=\sqrt{K / m}$.

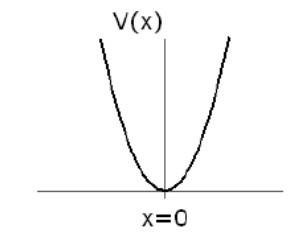

Simple Harmonic Oscillator

Questions 20-23 refer to System III shown above:

20. Choose all of the following statements that are correct about system III with a definite energy:

(1) Although classically the particle has the highest probability of being found at its (classical) turning points, for the quantum mechanical ground state, it is most likely to be found at the center of the well.

(2) The probability of finding the particle at the center of the well is zero for the first excited state.

(3) The probability of finding the particle is zero in the regions where the energy $E$ of the particle is less than $V(x)$.
A. 1 only
B. 2 only
C. 1 and 2 only
D. 2 and 3 only
E. all of the above

21. Consider two particles A and B in independent 1D simple harmonic oscillator potential energy wells, both identical to System III. Choose all of the following statements that are correct.

(1) If the expectation value of the momentum of particle $\mathrm{A}$ is larger than that for particle $\mathrm{B}$ $\left(\left\langle p_{A}\right\rangle>\left\langle p_{B}\right\rangle\right)$, the uncertainty in the position of particle $\mathrm{A}$ is smaller than that for particle

$$
\mathrm{B}\left(\Delta x_{A}<\Delta x_{B}\right) .
$$

(2) If the expectation value of the momentum of particle $\mathrm{A}$ is larger than that for particle $\mathrm{B}$ $\left(\left\langle p_{A}\right\rangle>\left\langle p_{B}\right\rangle\right)$, the uncertainty in the momentum of particle $\mathrm{A}$ is larger than that for particle B $\left(\Delta p_{A}>\Delta p_{B}\right)$.

(3) If the expectation value of the position of particle $\mathrm{A}$ is larger than that for particle $\mathrm{B}$ $\left(\left\langle x_{A}\right\rangle>\left\langle x_{B}\right\rangle\right)$, the expectation value of the momentum of particle $\mathrm{A}$ is smaller than that for particle $\mathrm{B}\left(\left\langle p_{A}\right\rangle<\left\langle p_{B}\right\rangle\right)$.
A. None of the above
B. 1 only
C. 2 only
D. 3 only
E. 1 and 3 only 
System III: A particle with mass $m$ interacts with a one-dimensional simple harmonic oscillator well $V(x)=K x^{2} / 2$ as shown. The stationary states are $\psi_{n}(x)$ and the allowed energies are $E_{n}=(n+1 / 2) \hbar \omega$ where $n=0,1,2,3, \ldots, \infty$ and $\omega=\sqrt{K / m}$.

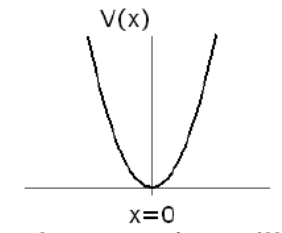

Simple Harmonic Oscillator

22. You prepare System III in an unknown superposition of $\psi_{0}$ and $\psi_{3}$. Choose all of the

following statements that are correct about how to experimentally estimate the probability distribution of the possible energies that can be measured in this state:

(1) Perform repeated measurements of the energy of the same particle a very large number of times to obtain the probability distribution.

(2) Perform a measurement of the particle's energy. Then, wait for a long time before measuring the energy again. Repeat this process a very large number of times on that particle to obtain the probability distribution.

(3) Perform a measurement of the particle's energy. Then, prepare the system again such that it has the original unknown superposition wave function. Then, measure the energy again. Repeat this process of preparation and measurement a very large number of times to obtain the probability distribution.
A. 1 only
B. 2 only
C. 3 only
D. 2 and 3 only
E. All of the above

23. Suppose at time $t=0$, System III is in the state $\frac{1}{\sqrt{2}}\left(\psi_{1}+\psi_{2}\right)$. Choose all of the following expectation values that depend on time.
(1) $\langle x\rangle$
(2) $\langle p\rangle$
(3) $\langle H\rangle$
A. 1 only
B. 2 only
C. 3 only
D. 1 and 2 only
E. all of the above

24. Choose all of the 1D potential energies shown in the figures below that allow both bound and scattering states.

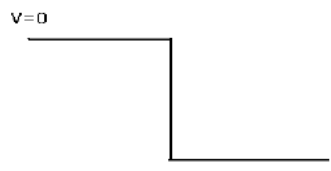

(1)

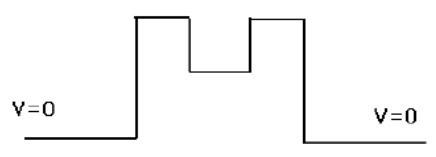

(3)
A. 1 only
B. 2 only
C. 2 and 4 only
D. 3 and 4 only
E. 2, 3 and 4 only

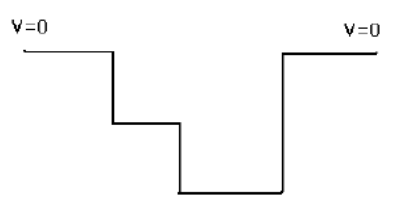

(2)

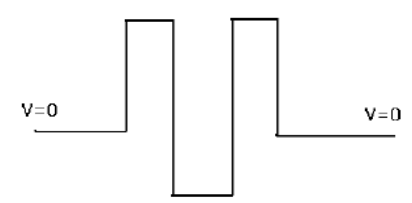

(4) 
25. $e^{i k x}$ is a stationary state wavefunction for a free particle in one dimension with momentum $p=\hbar k$. If the wavefunction of a free particle is $e^{i k x}+e^{-i k x}$, choose all of the following statements that are correct. (Ignore the wavefunction normalization issues in this problem.)

(1) The particle is still in a stationary state.

(2) The expectation value of the momentum of the particle is zero.

(3) The expectation value of the energy of the particle is zero.
A. 1 only
B. 2 only
C. 3 only
D. 1 and 2 only
E. 1 and 3 only

26. Choose all of the following equations that are correct for a quantum particle interacting with a 1D potential energy $V(x)$. (If you have learned both the Schrödinger \& Heisenberg formalisms of quantum mechanics, use the Schrödinger formalism.)

(1) $\frac{d \hat{x}}{d t}=\frac{\hat{p}}{m}$

$$
\begin{aligned}
& \frac{d \hat{p}}{d t}=-\frac{\partial V(\hat{x})}{\partial x} \\
& \hat{H}=\frac{\hat{p}^{2}}{2 m}+V(\hat{x})
\end{aligned}
$$
A. 3 only
B. 1 and 2 only
C. 1 and 3 only
C. 2 and 3 only
E. All of the above

27. Choose all of the following one dimensional Hamiltonian operators that have ONLY a discrete energy spectrum.
(1) $\hat{H}=-\frac{\hbar^{2}}{2 m} \frac{\partial^{2}}{\partial x^{2}}$ for all $x$.
(2) $\hat{H}=-\frac{\hbar^{2}}{2 m} \frac{\partial^{2}}{\partial x^{2}}+\frac{K}{2} x^{2}$ for all $x$ where $K$ is a positive constant.
(3) $\hat{H}=-\frac{\hbar^{2}}{2 m} \frac{\partial^{2}}{\partial x^{2}}+V(x), V(x)<0$ (but finite) when $0<x<a$ and $V(x)=0$ otherwise.
A. 1 only
B. 2 only
C. 2 and 3 only
D. all of the above
E. none of the above

28. Choose all of the following statements that are correct:

(1) The stationary states refer to the eigenstates of any operator corresponding to any physical observable.

(2) In an isolated system, if a particle is in a position eigenstate (has a definite value of position) at time $t=0$, the position of the particle is well-defined at all times $t>0$.

(3) In an isolated system, if a system is in an energy eigenstate (it has a definite energy) at time $t=0$, the energy of the particle is well-defined at all times $t>0$.
A. 1 only
B. 3 only
C. 1 and 3 only
D. 2 and 3 only
E. All of the above 
29. Choose all of the following statements that are correct about the Hamiltonian operator for a quantum system with potential energy $V=V(x)$.

(1) The Hamiltonian governs the time evolution of the quantum system.

(2) The Hamiltonian determines the form of the stationary state wavefunctions $\psi_{n}(x)$.

(3) The Hamiltonian determines the shape of the position eigenfunction (wavefunction for which the position of the particle has a definite value).
A. 1 only
B. 2 only
C. 1 and 2 only
D. 2 and 3 only
E. All of the above

30. A particle is interacting with a 1D potential energy well $V(x)$. If $V(x)$ is an even function, choose the correct statement about any possible wavefunction $\Psi(x, t)$ (not necessarily a stationary state) for the system at a specific time $t$.

A. $\Psi(x, t)$ must be even.

B. $\Psi(x, t)$ must be odd.

C. $\Psi(x, t)$ must be symmetric, but the symmetry axis is not necessarily about $x=0$.

D. $\Psi(x, t)$ must be even or odd, and no other possibility is allowed.

E. None of the above

31. A particle interacts with a 1D finite potential energy barrier as shown below. Two possible energies $E_{1}$ and $E_{2}$ are such that $0<E_{1}<V_{0}$ and $E_{2}>V_{0}$. Choose all of the following statements that are correct.

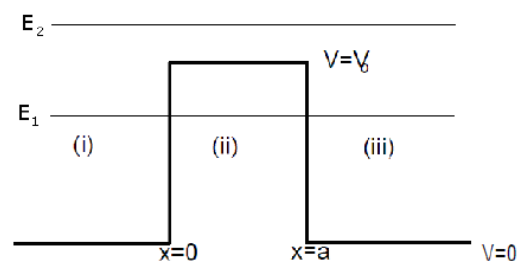

(1) A particle with energy $E_{1}$ is in a bound state between $x=0$ and $x=a$ and in a scattering state elsewhere.

(2) A particle with energy $E_{2}$ can be in a bound or a scattering state depending on its location.

(3) The energy $E_{2}$ corresponds to a particle in a scattering state and the energy $E_{1}$ corresponds to a particle in a bound state.
A. none of the above
B. 1 only
C. 2 only
D. 3 only
E. 1 and 2 only 